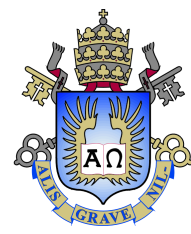

Cyntia Freitas Azevedo

\title{
Expectations and the coordination of monetary and fiscal policies
}

Tese de Doutorado

Thesis presented to the Programa de Pós-graduação em Economia of PUC-Rio in partial fulfillment of the requirements for the degree of Doutor em Economia.

Advisor: Prof. Tiago Couto Berriel 


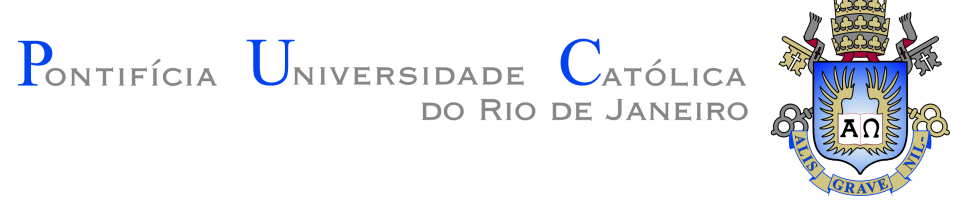

Cyntia Freitas Azevedo

\section{Expectations and the coordination of monetary and fiscal policies}

Thesis presented to the Programa de Pós-graduação em Economia of PUC-Rio in partial fulfillment of the requirements for the degree of Doutor em Economia. Approved by the undersigned Examination Committee.

Prof. Tiago Couto Berriel

Advisor

Departamento de Economia - PUC-Rio

Prof. Carlos Viana de Carvalho

Departamento de Economia - PUC-Rio

Prof. Eduardo Zilberman

Departamento de Economia - PUC-Rio

Dr. Eduardo H. de M. M. Loyo

Diretoria - Banco BTG Pactual

Dr. André Minella

Departamento de Pesquisas - Banco Central do Brasil

Prof. Augusto Cesar Pinheiro da Silva

Vice Dean of the Centro de Ciências Sociais - PUC-Rio

Rio de Janeiro, November the 23rd, 2018 
All rights reserved.

\section{Cyntia Freitas Azevedo}

Majored in Economics by Universidade de Brasília, holds a Masters degree in Economics by Universidade de Brasília and a MPhil degree in Economics by Columbia University, now holds a $\mathrm{PhD}$ degree in Economics from PUC-Rio.

Bibliographic data

Azevedo, Cyntia Freitas

Expectations and the coordination of monetary and fiscal policies / Cyntia Freitas Azevedo; advisor: Tiago Couto Berriel. - Rio de janeiro: PUC-Rio, Departamento de Economia, 2018.

v., 156 f: il. color. ; $30 \mathrm{~cm}$

Tese (Doutorado) - Pontifícia Universidade Católica do Rio de Janeiro, Departamento de Economia.

Inclui bibliografia

1. Economia - Teses;. 2. Expectativas;. 3. Política Monetária;. 4. Política Fiscal;. 5. Coordenação de políticas;. 6. Mudança de regimes;. 7. Multiplicadores fiscais;. 8. Taxa de juros zero.. I. Berriel, Tiago Couto. II. Pontifícia Universidade Católica do Rio de Janeiro. Departamento de Economia. III. Título. 
To my parents, for all the love, support and encouragement. 


\section{Acknowledgments}

This was a long journey and it is challenging to summarize in one page the long list of acknowledgements that deserve to be made. I need to start expressing my deepest gratitude to my advisor, Tiago Berriel. He was a friend, before being my advisor. Tiago and Cecília were on my side since the very first step of this journey and their example and support were crucial for me to get here. Tiago, I am very proud to have your name up there as my advisor. Thank you for believing this was possible and for finding space for me in your insane schedule.

I am deeply grateful to my family for always being on my side with love, encouragement and patience. You were responsible for building the foundations on my way here.

To Marco Aurélio, thank you for your support and encouragement. You were an important part of this journey and a true inspiration of persistence. Thank you for not letting me give up.

I am thankful for the support from Banco Central do Brasil and for the colleagues that got involved and inspired me. I was lucky to work with some of the best people there and to have very encouraging bosses. Loyo, Eduardo Fernandes, Adriana, Eduardo Lima, Minella, Angelo, Flávio, Brandt e Fábio, thank you for the patience and support. The teams from Depep and Deban, your support was crucial to get me here. The team from Conep, thank you for all the patience and comprehension, especially in these past months. Direx's staff, especially Tati and Vivi, thank you for always helping me find a space in Tiago's tight schedule.

Thank you to a group of strong and admiring women that I am lucky to have on my side. Ceci, Fábia, Iara, Nani, Telma, Thati, Tuti and Zhanna, I was blessed to receive your encouragement, inspiration and support, not only on this journey, but through life.

Alex McQuoid, thank you for not giving up on me. I miss our late hours at the library, all our coffee breaks and Sunday waffles. For sure, you made those days much lighter.

I am thankful PUC-Rio for letting me finish my journey there and the Economics department's staff, especially Graça and Ana Paula, for helping me while I was working in Brasília most of the time.

Thank you to my committee members for their participation and contributions to improve this work.

This study was financed in part by the Coordenação de Aperfeiçoamento de Pessoal do Nível Superior - Brasil (CAPES) - Finance Code 001. 


\section{Abstract}

Azevedo, Cyntia Freitas; Berriel, Tiago Couto (Advisor). Expectations and the coordination of monetary and fiscal policies. Rio de Janeiro, 2018. 156p. Tese de Doutorado Departamento de Economia, Pontifícia Universidade Católica do Rio de Janeiro.

This thesis discusses the role of agents' expectations regarding the conduction of monetary and fiscal policies in determining policy outcomes, economic dynamics and the volatilities of macroeconomic variables. The first Chapter shows that accounting for agents' expectations of a possible regime change has critical effects in the responses of macroeconomic variables to shocks, even if this switch does not materialize itself along the path observed after the shock. Recognizing the possibility of regime switches also have important consequences for the volatilities of endogenous variables, which are higher than those obtained in the linear model and very dependent on the policy parameters chosen by monetary and fiscal authorities in each regime. In the second Chapter, I discuss the role of expectations in determining the depth of a crisis when the economy hits the zero lower bound on nominal interest rates. I show that when analysing the impact of a fiscal stimulus during a zero interest rate episode, there is more than just short-run multipliers. To have larger positive effects on output and inflation, monetary and fiscal policies should last longer than the duration of the shock and be coordinated in their actions. The third Chapter presents a thoughtful evaluation of a fiscal stimulus in terms of the implied welfare losses making clear that it should account not only for the effects of policies on short-run output and inflation, but also for the present discounted value of output and inflation in future periods as well. It also analyses how to obtain the optimal level for the nominal interest rate once the economy gets out of the crisis state, if the monetary authority wants to use the expectations channel to undermine the depth of the crisis.

\section{Keywords}

Economics - Dissertation; Expectations; Monetary Policy; Fiscal Policy; Policy coordination; Regime Switching; Fiscal Multipliers; Zero interest rate. 


\section{Resumo}

Azevedo, Cyntia Freitas; Berriel, Tiago Couto. Expectativas e a coordenação das políticas monetária e fiscal. Rio de Janeiro, 2018. 156p. Tese de Doutorado - Departamento de Economia, Pontifícia Universidade Católica do Rio de Janeiro.

Essa tese discute o papel das expectativas dos agentes a respeito da condução das políticas monetária e fiscal na determinação dos efeitos dessas políticas, na dinâmica da economia e na volatilidades das variáveis macroeconômicas. O primeiro capítulo mostra que considerar as expectativas dos agentes a respeito de possíveis mudanças de regime tem efeitos importantes nas respostas das variáveis macroeconômicas aos choques, mesmo que essa mudança de regime não se materialize ao longo da trajetória observada após o choque. O reconhecimento da possibilidade de mudanças de regime também tem consequências importantes para a volatilidade das variáveis endógenas que são mais altas do que as obtidas no modelo linear e muito dependentes dos parâmetros de política escolhidos pelas autoridades fiscal e monetária em cada regime. O segundo capítulo discute o papel das expectativas a respeito das políticas futuras na determinação da profundidade de uma crise quando a economia atinge o limite inferior de zero para as taxas de juros nominais. Ele mostra que ao analisar o impacto de um estímulo fiscal durante um episódios de taxa de juros zero, deve-se olhar para além dos multiplicadores no curto prazo. Para ter efeitos positivos maiores, as políticas monetária e fiscal devem durar mais do que a crise e precisam ser coordenadas. O terceiro capítulo apresenta uma avaliação dos estímulos fiscais em termos das perdas de bem-estar, tornando claro que essa avaliação deve considerar não apenas o efeitos das políticas sobre a inflação e o produto no curto prazo, mas também o valor presente descontado da inflação e do produto nos períodos futuros. Ele também apresenta uma análise de como se obtém o nível ótimo da taxa de juros nominal uma vez que a economia não está mais em crise se a autoridade monetária pretende usar o canal das expectativas para reduzir a profundidade da crise.

\section{Palavras-chave}

Economia - Teses; Expectativas; Política Monetária; Política Fiscal; Coordenação de políticas; Mudança de regimes; Multiplicadores fiscais; Taxa de juros zero. 


\section{Table of contents}

1 Expectational Effects and Macroeconomic Dynamics with Switching Monetary and Fiscal Policies $\quad 13$

$\begin{array}{lll}1.1 & \text { Introduction } & 13\end{array}$

$\begin{array}{lll}1.2 & \text { Related Literature } & 15\end{array}$

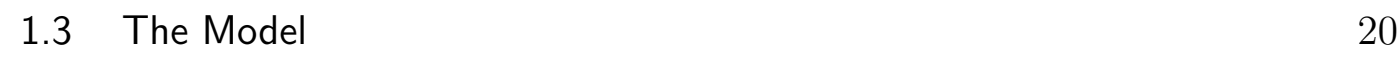

1.3.1 MSRE Model Solution and Determinacy 23

1.4 Impulse Responses Analysis 26

1.4.1 Impulse Responses to a Monetary Policy Shock 27

1.4.2 Impulse Responses to a Fiscal Policy Shock 30

1.4.3 Impulse Responses to Preference and Cost Push Shocks 32

1.5 Impact of Policy Responses in the Volatility of Macroeconomic Variables 34

1.6 Conclusion 40

2 Fiscal Stimulus at the Zero Lower Bound: The Role of Expectations and Policy Coordination $\quad 41$

2.1 Introduction 41

2.2 Related Literature 44

2.3 The Model 47

2.3.1 Revisiting the Literature - Two-state economy 49

2.3.2 The Three-State Economy 51

2.4 Solution Allocations 54

2.5 Analysis of Fiscal Policy Multipliers 60

2.5.1 Government Spending Multipliers 62

2.5.2 Income Tax Multipliers 65

2.6 Analysis of Different Policies' Combinations 67

2.6.1 Impact of Government Spending Stimulus 69

2.6.2 Impact of Income Taxes Stimulus 73

$\begin{array}{lll}2.7 & \text { Conclusion } & 76\end{array}$

3 Fiscal Stimulus at the Zero Lower Bound: Welfare Analysis and Optimal $\begin{array}{ll}\text { Transitional State Monetary Policy } & 78\end{array}$

$\begin{array}{lll}3.1 & \text { Introduction } & 78\end{array}$

$\begin{array}{lll}\text { 3.2 Welfare Loss Function } & 79\end{array}$

3.3 Optimal Transitional State Monetary Policy 81

3.4 Welfare Analysis of Monetary and Fiscal Policies Combinations 83

3.4.1 Impact of Government Spending Stimulus 85

3.4.2 Impact of Income Taxes Stimulus 90

3.5 Conclusion 93

$\begin{array}{ll}\text { Bibliography } & 95\end{array}$

A Appendix to Chapter $1 \quad 99$

A.1 Economic Outlook and Expectations in 2015-2016 99

$\begin{array}{lll}\text { A.2 IRFs varying }\left(p_{11}, p_{22}\right) & 101\end{array}$ 
A.3 The Forward Method for Solving MSRE models

A.3.1 Necessary and Sufficient Conditions for Determinacy of the Forward Solution

A.4 Impulse Response Functions in the MSRE-FreeCase 112

A.5 Impulse Response Functions in the MSRE-Fixed Case 113

$\begin{array}{lll}\text { A.6 Conditional and Unconditional Volatilities } & 117\end{array}$

B Appendix to Chapter 2 120

B.1 Proof of Proposition 2.1 120

B.2 Proof of Proposition 2.2 135

C Appendix to Chapter $3 \quad 144$

C.1 Proof of Proposition $3.1 \quad 144$

C.2 Proof of Proposition 3.2 


\section{List of figures}

$\begin{array}{lll}\text { Figure 1.1 Determinacy Regions } & 25\end{array}$

$\begin{array}{lll}\text { Figure 1.2 IRFs to a Monetary Policy Shock } & 28\end{array}$

Figure 1.3 IRFs to a Fiscal Policy Shock 31

Figure 1.4 IRFs to a Cost Push Shock 33

$\begin{array}{lll}\text { Figure 1.5 } & \text { IRFs to a Preference Shock } & 34\end{array}$

Figure 1.6 Standard deviations as a function of $\phi_{\pi, 1} \quad 36$

Figure 1.7 Standard deviations as a function of $\psi_{b, 1} \quad 37$

Figure 1.8 Standard deviations as a function of $\phi_{\pi, 2} \quad 38$

Figure 1.9 Standard deviations as a function of $\psi_{b, 2} \quad 39$

Figure 2.1 Benchmark Case - Two-state economy 50

Figure 2.2 Transitional State Case - Three-state economy 52

Figure 2.3 Short-run coefficients as a function of probability $\mu \quad 62$

Figure 2.4 Shock and interest rate coefficients as a function of $b \quad 63$

Figure 2.5 Government Spending multipliers as a function of $b \quad 65$

Figure 2.6 Taxes multipliers as a function of $b \quad 67$

Figure 2.7 Short- and medium-run impact of government spending $\begin{array}{ll}\text { stimulus } & 71\end{array}$

Figure 2.8 Short-Run Impact of Temporary Income Tax Stimulus 74

Figure 3.1 Welfare losses and optimal transitional state monetary policy with government spending stimulus

Figure 3.2 Optimal medium-run nominal interest rate $\left(i_{M}^{*}\right)$ as a function of $\mu$ and $b$

Figure 3.3 Short- and medium-run output and inflation with optimal transitional state monetary policy and government spending stimulus

Figure 3.4 Welfare losses and optimal transitional state monetary policy with income tax stimulus

Figure 3.5 Short- and medium-run output and inflation with optimal transitional state monetary policy and income taxes stimulus 92

$\begin{array}{llr}\text { Figure A.1 Fiscal Policy Deterioration } & 99\end{array}$

Figure A.2 Inflation Expectations Desanchoring 100

$\begin{array}{lll}\text { Figure A.3 Recession Deepening } & 100\end{array}$

Figure A.4 IRFs to a Monetary Policy Shock- AM/PF Regime 101

Figure A.5 IRFs to a Monetary Policy Shock- PM/AF Regime 102

Figure A.6 IRFs to a Fiscal Policy Shock- AM/PF Regime 103

Figure A.7 IRFs to a Fiscal Policy Shock- PM/AF Regime 104

Figure A.8 IRFs to a Cost-Push Shock- AM/PF Regime 105

Figure A.9 IRFs to a Cost-Push Shock- PM/AF Regime 106

Figure A.10 IRFs to a Preference Shock- AM/PF Regime 107

Figure A.11 IRFs to a Preference Shock- PM/AF Regime 108 


\section{List of tables}

Table 1.1 Determinacy and the coordination between monetary and fiscal policy regimes

Table 2.1 Summary of possible policy choices

Table 2.2 Analytical expressions for medium-run solution coefficients from Proposition 2.1

Table 2.3 Analytical expressions for short-run solution coefficients from Proposition 2.1

Table 2.4 Analytical expressions for the coefficients in Condition (C4) from Proposition 2.1

Table 2.5 Analytical expressions for medium-run solution coefficients from Proposition 2.2

Table 2.6 Analytical expressions for short-run solution coefficients from Proposition 2.2

Table 2.7 Analytical expressions for the coefficients in Condition (C4') from Proposition 2.2

Table 2.8 Solution coefficients in each state as a function of $b \quad 61$

Table 2.9 Short- and medium-run output and inflation allocations

Table 3.1 Analytical expressions for optimal nominal interest rate coefficients - Proposition 3.2

Table 3.2 Short- and medium-run output and inflation allocations and welfare losses 


\section{List of Abreviations}

NK - New Keynesian

LRE - Linear Rational Expectations

MSRE - Markov-Switching Rational Expectations

FTPL - Fiscal Theory of the Price Level

LRTP - Long-run Taylor Principle

AM - Active Monetary Policy

PM - Passive Monetary Policy

AF - Active Fiscal Policy

PF - Passive Fiscal Policy

ZLB - Zero Lower Bound 


\section{1 \\ Expectational Effects and Macroeconomic Dynamics with Switching Monetary and Fiscal Policies}

\section{1 \\ Introduction}

In traditional macroeconomic models, it is usual to assume that monetary policy stabilizes inflation, while fiscal policy ensures government's solvency and stabilizes debt. This type of model is known as being characterized by a Ricardian regime or a regime of monetary dominance, ${ }^{1}$ in which fiscal policy plays no role in determining the dynamics of macroeconomic variables other than debt. On the other hand, there has been much discussion regarding time spells along history in which some economies would be better characterized by a model under a non-Ricardian regime or a regime of fiscal dominance ${ }^{2}$. In such a model, monetary policy is no longer able to determine inflation independently from the fiscal policy stance. There is an interaction between these policies that determines the price level, stabilizes debt and ensures government's solvency.

Although hard to identify empirically, the possibility of the Brazilian economy displaying fiscal dominance in certain periods has been the source of analysis in the literature and worries to policy makers, especially when the fiscal situation deteriorates and doubts regarding debt solvency arise. Blanchard (7) shows some evidence that this might have been the case in 2002-2003, and worries about its resurgence gained ground in 2014-2015.

At that time, the Brazilian economy was showing signs of deepening recession, rising inflation and worsening of the fiscal scenario, with increasing primary deficits and government debt. Meanwhile, even with consecutive increases in the nominal interest rate, inflation was rising, while output was moving towards a deep recession. Expectations started to deteriorate and agents were getting very pessimistic regarding future prospects of the economy. ${ }^{3}$ This scenario started to raise concerns about the effectiveness of monetary policy in fighting inflation and how fiscal policy could be limiting the acting of the monetary authority. There was a heated discussion among Brazilian economists

\footnotetext{
${ }^{1}$ For textbook treatments refer to Galí (1) and Woodford (2).

${ }^{2}$ See Sargent \& Wallace (3), Loyo (4), Woodford (5) and Cochrane (6).

${ }^{3}$ The graphs in Appendix A.1 illustrate this scenario.
} 
with respect to the characterization of the monetary/fiscal regime that prevailed at that time and what policy measures should the authorities adopt (see, for example, Berriel (8), de Bolle (9), Schymura (10), Berriel (11) and Carvalho (12)). ${ }^{4}$

This uncertainty regarding the prevailing regime complicates policy prescriptions, since under fiscal dominance the signs expected for monetary policy actions are reverted. An increase in nominal interest rate leads to more inflation, not less, as expected in a regime of monetary dominance.

One aspect that was barely discussed at the time is how expectations that a switch to a regime of fiscal dominance might occur would affect policy outcomes observed in the economy. A key feature of mainstream macroeconomic models, and of the inflation targeting regime adopted in many countries, is the role of agents' expectations. It is known that monetary and fiscal policy outcomes are different depending on how they coordinate (see Leeper (14)) and that agents' expectations regarding future policy actions also affect macroeconomic dynamics (this is discussed, for example, in Liu et al. (15)). But would an expected regime switch that does not materialize itself affect this dynamics as well?

The goal of this Chapter is then to characterize, in a model in which monetary and fiscal policies are allowed to switch between different regimes, how the dynamics of macroeconomic variables and their volatilities might be influenced by expectations of regime changes in the coordination between monetary and fiscal authorities. In order to perform this analysis I use a calibrated simple New Keynesian (NK) model with monetary and fiscal policy rules and compare the results obtained under the Linear Rational Expectations (LRE) hypothesis, with those under the Markov Switching Rational Expectations (MSRE) hypothesis. In the LRE model, parameters are constant, while in the MSRE model the parameters that govern monetary and fiscal policies' stances are allowed to switch between regimes of monetary and fiscal dominance, with probabilities associated with each regime.

I follow Cho \& Moreno (16) and apply the method introduced by Cho (17) to obtain the MSRE model solution. His method provides very tractable and easy to verify determinacy conditions for a general class of MSRE models with lagged variables. This is crucial for permitting the analysis of regime switching in the stance of fiscal policy since it is necessary to take into account the government's budget constraint and a fiscal rule in which debt

\footnotetext{
${ }^{4}$ With the exception of de Bolle (9), the other articles are only available in portuguese. There were a number of other articles in the press about this discussion, which are available in portuguese as well. One great treatment of this theme occurred in a Seminar organized by Insper (13) (in portuguese).
} 
is predetermined.

Then, I compare the impulse responses obtained in the LRE model, with those from he MSRE model. In the latter, it is necessary to assume not only the regime observed when the shock hits the economy, but also how regimes evolve along the impulse response path. I analyze two cases, the first being the one where regimes switch freely along the path (MSRE-free case). In the second case, though being allowed to switch, the regime observed on impact does not change during the impulse response trajectory under analysis (MSREfixed case). I also compare the macroeconomic variables' volatilities in the LRE and MSRE models and how they depend on the policy rule parameters in each regime.

I find that accounting for agents' expectations of a possible regime switching has critical effects in the responses of macroeconomic variables to shocks, even if this switch does not materialize itself along the path observed after the shock. In this case, I obtain the striking result that, if the economy is under a regime of monetary dominance when hit by a monetary or a fiscal policy shock, it can exhibit persistent stagflation if there is a high probability of moving to a regime of fiscal dominance.

Additionally, recognizing the possibility of regime switches has important consequences for the volatilities of endogenous variables which in general are higher than those obtained in the LRE model. Besides, these volatilities are very dependent on the policy parameters chosen by monetary and fiscal authorities in both regimes.

The next Section places this work in the related literature. Section 1.3 introduces the model used in the analysis, its parametrization, how its solution is obtained and the results in terms of its determinacy. Section 1.4 examines the responses of the endogenous variables to the various shocks in the MSRE model, comparing with those obtained under the LRE model. These two models are also compared when analyzing the behavior of macroeconomic variables' volatilities in Section 1.5. Section 1.6 concludes the Chapter.

\section{2}

\section{Related Literature}

There has been some confusion regarding the definition of fiscal dominance since it was first introduced in the seminal paper of Sargent \& Wallace (3). The concept is directly related to the intertemporal government budget constraint that is assumed to be an always valid equilibrium condition. As pointed out by Cochrane (6), in a very generic formulation, this constraint can be seen as a valuation equation 


$$
\frac{\text { Nominal Gov't Debt }}{\text { Price Level }}=\text { Expected PV of primary surpluses, }
$$

which relates the real value of debt to the expected present value of primary surpluses. In general, in a regime of monetary dominance, the government adjusts its surpluses through fiscal policy, leaving this equation in the background of monetary policy. With this setup, the budget constraint is satisfied for any price level set by the monetary authority.

The regime of fiscal dominance emerges when agents start doubting that there will be enough surpluses in the future to stabilize debt, which leads to the need for adjustments through other mechanisms. The mechanism explored in Sargent \& Wallace (3) makes this adjustment through increases in seigniorage, generating extra revenues that can balance the expected primary surpluses, but also causing prices to raise.

Nevertheless, this is not the only mechanism through which it is possible to have a regime of fiscal dominance. Even a model without money can display fiscal dominance. This is the mechanism explored in the Fiscal Theory of the Price Level (FTPL) by Sims (18), Woodford (5) and Cochrane (6), among others. In their setup, the adjustment in equation (1-1) happens through the revaluation of $\mathrm{debt}^{5}$ as the result of an inflationary process, which adjusts the real level of debt. In this case, price dynamics is also determined by the intertemporal government budget constraint, not only by monetary policy.

Woodford (5) explains how this increase in prices is a consequence of a wealth effect that makes households fell richer in anticipation of lower primary government surpluses in the future which are not expected to be restored through increases in taxes. This leads to an increase in the demand for goods and services, and consequently an increase in prices as well. When prices rise, there is a reduction in the real value of nominal assets, restoring the equilibrium in the valuation equation.

The FTPL argues that in this environment, if the monetary authority raises the nominal interest rate to fight the increase in inflation, it generates paradoxical effects, causing more, not less inflation. This happens because the increase in the nominal rate also increases debt services and, consequently, its stock. With the numerator in the left-hand side of equation (1-1) increasing faster, prices need to raise even more to stabilize it.

It is worth highlighting that, although being an important sign of fiscal deterioration, a very high current level of debt is not required for the economy to display fiscal dominance. What matters are expectations regarding the

\footnotetext{
${ }^{5}$ Note that it is necessary that a relevant part of debt to be nominal for this mechanism to work.
} 
government's ability to generate primary surpluses in the future to pay for this debt.

Another explanation for this paradoxical effect of monetary policy on prices was presented by Blanchard (7). In his model, the mechanism that leads to more inflation in response to an increase in the nominal rate is different from that presented in the FTPL. He argues that if the economy is under a regime of fiscal dominance and the monetary authority raises the nominal interest rate, it worsens debt dynamics and the risk perception of domestic assets. This causes a decrease in the demand for these assets and a migration to other assets as foreign debt, for example. As a consequence of this flow of investments to other countries, the exchange rate depreciates generating an increase in prices. ${ }^{6}$

An essential contribution to the discussion about the coordination between monetary and fiscal policies was given by Leeper (14). He introduced a taxonomy much used in the literature that defines monetary dominance as a regime where monetary policy is active and fiscal policy passive (AM/PF), while fiscal dominance is characterized by a passive monetary policy and active fiscal policy $(\mathrm{PM} / \mathrm{AF}) .{ }^{7}$ The passive policy is the one that guarantees the satisfaction of the intertemporal government budget constraint. An important result of his work is the characterization of determinacy in a simple NK model with monetary and fiscal policy rules. Briefly, the model will have a determinate solution if one authority is passive and the other active. If both authorities are passive, there is no solution, while if both are active, the solution is indeterminate. $^{8}$

Since Leeper's work, the literature on the interactions between monetary and fiscal policies has grown significantly. However, for a while, most analyses were based on the characterization of each regime independently. The use of MSRE models allows extending these analyses, bringing the possibility of accounting for agents' expectations regarding recurrent changes between regimes.

The issue of how the possibility of regime switching in monetary policy affects the determinacy of equilibrium, and the macroeconomic dynamics was discussed in Davig \& Leeper (19). They generalize the Taylor Principle ${ }^{9}$ to an environment in which the reaction coefficients in the monetary policy

${ }^{6}$ This assumes an effective passthrough from the exchange rate to prices.

${ }^{7}$ Throughout this Chapter I use the terms regime of fiscal dominance, PM/AF regime and non-Ricardian regime interchangeably. In the same sense, we also use the terms regime of monetary dominance, $\mathrm{PM} / \mathrm{AF}$ regime and ricardian regime as equivalent.

${ }^{8}$ Section 1.3 explains what this means in terms of the model.

${ }^{9}$ Proposition that central banks can stabilize the macroeconomy by raising their interest rate instrument more than one-for-one in response to higher inflation. In many monetary models, this condition is necessary and sufficient for the existence of a determinate rational expectations equilibrium. 
rule evolve according to a Markov process. They examine how this variation of coefficients over time affects the nature of equilibrium in popular models of monetary policy and derive what they call a Long-Run Taylor Principle (LRTP) that delivers a determinate equilibrium. Their main result is that policy can satisfy the Taylor Principle in the long run, even while deviating from it substantially for brief periods or modestly for prolonged periods. LRTP for the New Keynesian (NK) model dramatically expands the determinacy region relative to the constant-parameter setup. Besides, the possibility of a regime change alters the qualitative and quantitative predictions of a conventional NK model, with the effects that arise from the possibility that future regimes may differ from the current regime changing the responses of inflation and output to exogenous disturbances in quantitatively important ways.

As pointed by Liu et. al. (15), in an economy where past changes in monetary policy rules are observable, and future changes are likely, rational agents' information sets should include a probability distribution over possible policy shifts in the future. These authors call expectation effect of regime shifts the difference between the equilibrium outcome from a model that ignores probabilistic shifts in future policy regime, and that from a model that takes into account such expected changes in regime ${ }^{10}$. They also analyse the magnitude and the asymmetry of the expectational effects in a MSRE model. In their setup, monetary policy is allowed to switch between hawkish (stronger response of interest rate to inflation) and dovish (weaker response of interest rate to inflation) regimes, where the latter does not necessarily represent a passive monetary policy (which would imply indeterminacy in the model), it can simply mean a less active policy. They find asymmetry in the expectation effect across regimes, with quantitatively more critical expectation effects under the dovish regime than those under the hawkish regime. Asymmetry exists even if monetary policy responds more than one-for-one to inflation in both regimes. They also show that the expectational effects affect inflation dynamics and that the volatility in each regime depends on the probability of switching to the other regime.

Both Davig \& Leeper (19) and Liu et. al. (15) place fiscal policy in the background, assuming lump-sum taxes and transfers to passively adjust to ensure solvency. Bhattarai et. al. (21) analyse inflation dynamics in a model with both monetary and fiscal policies and discuss how it critically depends on whether these policies are active or passive. Their analysis does not allow for regime switches, but they show how the coordination between policy

\footnotetext{
${ }^{10}$ These effects were first discussed in Leeper \& Zha (20).
} 
authorities can significantly affect the responses of inflation to shocks. Under an $\mathrm{AM} / \mathrm{PF}$ policy regime, where a high response of interest rates to inflation is coupled with a high response of taxes to public debt, monetary policy controls inflation dynamics, while fiscal policy plays no role. They illustrate the classic FTPL mechanism. Under a PM/AF policy regime, where a low response of interest rates to inflation is coupled with a low response of taxes to public debt, public debt is inflationary. In this regime, this happens because monetary policy increases the interest rate in response to a reduction in the inflation target. This causes an increase in debt, which households perceive as an increase in wealth not matched by increases in taxes that are enough to satisfy the government budget constraint at the prevailing prices (positive wealth effect). Thus, households' spending increases, consequently increasing inflation as well, moving it in the opposite direction from the target.

Cho \& Moreno (16) examine a MSRE model in which both monetary and fiscal policies are allowed to switch between active and passive stances. They use this model to analyse how accounting for the possibility of regime changes impacts its determinacy and derive combinations of regime-switching monetary and fiscal policies that imply in determinate solutions. They point out that determinacy / indeterminacy / no stable solution regions are qualitatively and quantitatively different from those implied by the same type of model when regimes are kept constant. Their work does not discuss how the expectational effects that arise when regimes are allowed to switch impact the dynamics of macroeconomic variables after a shock hits the economy.

Cho \& Moreno (16) do not discuss the possibility of regime-dependent volatilities since there is no impact for the model's determinacy of leaving it aside. However, Bianchi \& Ilut (22) and Baele et. al. (23) and obtain interesting results when estimating MSRE models that allow for regimeswitching volatilities in order to explain the Great Moderation in the US.

Obtaining the solutions in MSRE models is more challenging than in LRE models since agents must be allowed to take into account the possibility of future regime changes when forming expectations. Various solution methods have been proposed in the literature. Farmer et. al. (24) develop a set of necessary and sufficient conditions for equilibria to be determinate in a class of forward-looking MSRE models. The paper provides such conditions for an important subset of MSRE model, those in which there are no predetermined variables. In their framework, the fundamental solution is always unique by construction. Thus, only the non-existence of Mean Square Stable sunspot components needs to be explored. Cho (17) shows that there is a dimensionality problem in their solution method, since the set of non-fundamental components 
is a $n^{2} S(S-1)$-dimensional solution space, where $S$ is the number of states and $n$ the number of endogenous variables. Therefore, the task of identifying determinacy by finding the whole solution space gets intractable.

Farmer et. al. (25) develop a method for deriving equilibria of a general class of MSRE models, including those with lagged variables, and a new algorithm for computing these equilibria. Their method can help in identifying indeterminacy by finding more than one stable solution. However, Cho (16) points out that the uniqueness of the Mean Square Stable fundamental solution, and therefore determinacy, cannot be verified in this way because there is no known proof that their algorithm can identify all of the fundamental solutions.

The main contribution of Cho (16) is to establish tractable sufficient conditions for determinacy in a class of MSRE models with predetermined variables when faced with a lack of information about the number of fundamental solutions and the large dimensionality of sunspot components. He also provides sufficient conditions for indeterminacy that are easy to verify. This method extends the forward method of Cho \& Moreno(18) developed for LRE models to MSRE models. In contrast with Farmer at. al. (25), this approach does not require obtaining all sunspot solutions to establish determinacy, nor does it require solving for all of the fundamental solutions, which are both difficult tasks in the MSRE context. I follow Cho \& Moreno (16) and apply Cho's method to obtain the solutions for the model discuss in the next Section.

\section{3}

\section{The Model}

In order to examine the dynamics of the economy with the possibility of regime switching in both monetary and fiscal policies, I adopt the same baseline macro model specification used by Cho \& Moreno (16) to study the issue of determinacy in this type of model. It is composed by a Phillips curve, an IS curve, a monetary policy rule, the government's budget constraint and a fiscal policy rule:

$$
\begin{aligned}
\pi_{t} & =\beta E_{t} \pi_{t+1}+\kappa y_{t}+z_{t}^{A S}, \\
y_{t} & =E_{t} y_{t+1}-\varphi\left(r_{t}-E_{t} \pi_{t+1}\right)+z_{t}^{I S}, \\
r_{t} & =\phi_{\pi}\left(s_{t}\right) \pi_{t}+z_{t}^{M P} \\
b_{t} & =\beta^{-1}\left(b_{t-1}-\tau_{t}\right)+\bar{b}\left(r_{t}-\beta^{-1} \pi_{t}\right)+z_{t}^{F P}, \\
\tau_{t} & =\psi_{b}\left(s_{t}\right) b_{t-1},
\end{aligned}
$$


where $\pi_{t}$ is the inflation rate, $y_{t}$ is the output gap, $r_{t}$ is the short-term monetary policy rate, $\tau_{t}$ is lump-sum taxes, and $b_{t}$ the government debt-over-GDP ratio multiplied by the gross interest rate. All these variables are defined as percentage deviations of their steady-state values, except $b_{t}$, whose deviations from its steady-state value $(\bar{b})$ is divided by GDP. The fixed parameters $\beta$, $\kappa$ and $\varphi$ are the time discount factor, the Phillips curve parameter ${ }^{11}$ and the inverse elasticity of substitution, respectively. $s_{t}$ is a $S$-states ergodic Markov chain with transition probability matrix $P$ for which the $(i, j)$-th element is $p_{i j}=\operatorname{Pr}\left(s_{t+1}=j \mid s_{t}=i\right)$ and $\sum_{j=1}^{S} p_{i j}=1$ for all $i, j \in\{1,2, \ldots, S\}$. $I_{t}=\left\{x_{t-l}, s_{t-l}, z_{t-l}, l=0,1,2, \ldots\right\}$ is the information set available at time $t$ and $E_{t}[] \equiv E\left[\cdot \mid I_{t}\right]$ is the mathematical expectation operator conditional on $I_{t}$. The shocks $z_{t}^{A S}, z_{t}^{I S}, z_{t}^{M P}$ and $z_{t}^{F P}$ represent cost-push, preference, monetary policy and fiscal policy exogenous shocks, respectively. These are independent autoregressive processes, with persistence $\rho_{h}$ and variance $\sigma_{h}, h \in$ $\{A S, I S, M P, F P\}$.

The regime-dependent parameter $\phi_{\pi}\left(s_{t}\right)$, which defines the response of nominal interest rate to inflation, characterizes the monetary policy stance. Likewise, the fiscal policy stance is characterized by the regime-dependent parameter $\psi_{b}\left(s_{t}\right)$, which defines the response of taxes to debt. A common taxonomy ${ }^{12}$, introduced in the literature by (14), defines monetary policy as active when $\phi_{\pi}\left(s_{t}\right)>1$, and passive otherwise. Fiscal policy stance is said to be active if $\psi_{b}\left(s_{t}\right) \leq 1-\beta$, and passive otherwise.

In a LRE model that considers monetary policy only and ignores the possibility of regime switching, $\phi_{\pi}>1$, a condition known as the Taylor Principle, is necessary and sufficient to establish determinacy of the model.

A well known result provided by Leeper (14) establishes that, with constant policy parameters $\phi_{\pi}$ and $\psi_{b}$, determinacy in a LRE model with monetary and fiscal policies depends on the coordination between the policy authorities. Table 1.1 summarizes the four sets of monetary/fiscal policy combinations and their implications in terms of determinacy of the solution in a model of the type formed by equations (1-2)-(1-6) with constant parameters. Working with a NK model without fiscal policy and regime switching in the monetary policy parameter, Davig \& Leeper (19) show how the possibility of a regime change can expand the determinacy region, relative to the constantparameter setup. They derive what they call a Long-Run Taylor Principle (LRTP) that delivers a unique bounded equilibria in regions of parameters' combination not observed in models where no change in parameters is allowed.

\footnotetext{
${ }^{11}$ This is given by $\kappa \equiv \frac{(1-\alpha)(1-\alpha \beta)}{\alpha}$, where $\alpha$ is the degree of price rigidity in the economy.

${ }^{12}$ This taxonomy is used independently of these parameters being regime-dependent or constant.
} 
Table 1.1: Determinacy and the coordination between monetary and fiscal policy regimes

\begin{tabular}{ccc}
\hline & $\begin{array}{c}\text { Active Fiscal Policy } \\
(\mathrm{AF}) \psi_{b} \leq 1-\beta\end{array}$ & $\begin{array}{c}\text { Passive Fiscal Policy } \\
(\mathrm{PF}) \psi_{b}>1-\beta\end{array}$ \\
\hline $\begin{array}{c}\text { Active Monetary Policy } \\
(\mathrm{AM}) \phi_{\pi}>1\end{array}$ & No stable solution & Determinacy \\
\hline $\begin{array}{c}\text { Passive Monetary Policy } \\
(\mathrm{PM}) \phi_{\pi} \leq 1\end{array}$ & Determinacy & Indeterminacy \\
\hline
\end{tabular}

They obtain that monetary policy can satisfy the Taylor Principle in the long run, even while deviating from it substantially for brief periods or modestly for prolonged periods.

Within the context of MSRE models, a particular concept of stability of solutions must be chosen because different concepts of stability yield different determinacy results. In their discussion on the Long Run Taylor Principle (LRTP), Davig \& Leeper (19) use the concept of boundedness. ${ }^{13}$ Farmer et. al. (24) use mean-square stability (MSS). ${ }^{14}$ Cho (17) argues in favour of using MSS because it allows characterizing determinacy for a larger set of stochastic processes that are covariance-stationary, not necessarily bounded, which is the case of normally distributed shocks used in most empirical studies and simulation exercises in macroeconomics. Besides, he argues that MSS leads to tractable conditions to establish determinacy for MSRE models.

Cho \& Moreno (16) derive the conditions for a LRTP in the MSS sense,i.e., the stochastic process must have finite first and second moments allowing the comparison with the determinacy regions implied by the LRTP under bounded equilibrium. They show that the MSS equilibrium in the MSRE model also expands the determinacy regions when compared with the constantparameter case. However, there are parameter combinations for which the LRTP under bounded equilibrium holds, but which do not satisfy the MSS equilibrium conditions.

They also analyse determinacy conditions in the model with regime switching in both monetary and fiscal policies like the one in (1-2)-(1-6). They discuss how these conditions depend on the parameters of the model and the probabilities associated with each regime. Although presenting an extensive

\footnotetext{
${ }^{13}$ Bounded stability requires bounded paths and thus rules out temporarily explosive paths. A sequence $\left\{s_{n}\right\}$ is said to be bounded if the range $\left\{s_{n}: n \in \mathbb{N}\right\}$ is a bounded set. That is, if there exists an $M \geq 0$ such that $\left|s_{n}\right| \leq M$ for all $n \in \mathbb{N}$.

${ }^{14} \mathrm{An} n \times 1$ stochastic process $y_{t}$ is mean-square stable (MSS) if there exists an $n \times 1$ vector $\bar{y}$ and an $n \times n$ matrix $Q$ such that $\lim _{t \rightarrow \infty}\left(E\left[y_{t}\right]-\bar{y}\right)=0_{n \times 1}$ and $\lim _{t \rightarrow \infty}\left(E\left[y_{t} y_{t}^{\prime}\right]-Q\right)=$ $0_{n \times n}$.
} 
discussion regarding determinacy of the MSRE model with regime switching in both monetary and fiscal policies, Cho \& Moreno (16) do not discuss the implications regarding macroeconomic dynamics and volatilities in this type of model. As mentioned in the introduction, accounting for the possibility of regime changes implies critical expectational effects in the responses of endogenous variables to shocks and their overall volatilities.

In what follows, I discuss the moedl's solution and determinacy conditions under the parametrization proposed.

\subsection{1}

\section{MSRE Model Solution and Determinacy}

In order to obtain the solution for the MSRE model in (1-2)-(1-6) using the forward method, it needs to be casted in the canonical form proposed by Cho (17). Let $x_{t}=\left[\pi_{t} y_{t} r_{t} b_{t}\right]^{\prime}$ be the vector of endogenous variables and $z_{t}=\left[\begin{array}{llll}z_{t}^{A S} & z_{t}^{I S} & z_{t}^{M P} & z_{t}^{F P}\end{array}\right]^{\prime}$ be the vector of exogenous variables at time $t$. After substituting the fiscal policy rule (1-6) into the government budget constraint (1-5), and collecting the coefficients, we can write model (1-2)-(1-6) as:

$$
\begin{gathered}
B_{1}\left(s_{t}\right) x_{t}=A_{1} E_{t} x_{t+1}+B_{2}\left(s_{t}\right) x_{t-1}+z_{t}, \\
z_{t}=R z_{t-1}+\epsilon_{t}, \quad \epsilon_{t} \sim\left(0_{m \times 1}, \Sigma\right) \\
B_{1}\left(s_{t}\right)=\left[\begin{array}{cccc}
1 & -\kappa & 0 & 0 \\
0 & 1 & \varphi & 0 \\
-\phi_{\pi}\left(s_{t}\right) & 0 & 1 & 0 \\
\bar{b} \beta^{-1} & 0 & -\bar{b} & 1
\end{array}\right], A_{1}=\left[\begin{array}{cccc}
\beta & 0 & 0 & 0 \\
\varphi & 1 & 0 & 0 \\
0 & 0 & 0 & 0 \\
0 & 0 & 0 & 0
\end{array}\right], \\
B_{2}\left(s_{t}\right)=\left[\begin{array}{cccc}
0 & 0 & 0 & 0 \\
0 & 0 & 0 & 0 \\
0 & 0 & 0 & 0 \\
0 & 0 & 0 & \beta^{-1}\left(1-\psi\left(s_{t}\right)\right)
\end{array}\right], R=\left[\begin{array}{cccc}
\rho_{A S} & 0 & 0 & 0 \\
0 & \rho_{I S} & 0 & 0 \\
0 & 0 & \rho_{M P} & 0 \\
0 & 0 & 0 & \rho_{F P}
\end{array}\right] \text { and } \\
\Sigma=\left[\begin{array}{ccccc}
\sigma_{A S}^{2} & 0 & 0 & 0 \\
0 & \sigma_{I S}^{2} & 0 & 0 \\
0 & 0 & \sigma_{M P}^{2} & 0 \\
0 & 0 & 0 & \sigma_{F P}^{2}
\end{array}\right] .
\end{gathered}
$$

Invert matrix $B_{1}\left(s_{t}\right)$ and define $A\left(s_{t}\right) \equiv B_{1}^{-1}\left(s_{t}\right) A_{1}, B\left(s_{t}\right) \equiv$ $B_{1}^{-1}\left(s_{t}\right) B_{2}\left(s_{t}\right)$ and $C\left(s_{t}\right) \equiv B_{1}^{-1}\left(s_{t}\right)$ to write the model in Cho's canonical 
form: ${ }^{15}$

$$
\begin{aligned}
& x_{t}=A\left(s_{t}\right) E_{t} x_{t+1}+B\left(s_{t}\right) x_{t-1}+C\left(s_{t}\right) z_{t}, \\
& z_{t}=R z_{t-1}+\epsilon_{t}, \quad \epsilon_{t} \sim\left(0_{m \times 1}, \Sigma\right) .
\end{aligned}
$$

With this, it is possible to obtain the forward solution and check the conditions to identify determinacy / indeterminacy / no-stable solutions for a range of parameters in the model. ${ }^{16}$

I set the regime-independent parameters to the same values used in Cho $\&$ Moreno (16): $\beta=0.99, \kappa=0.1, \varphi=1$ and $\bar{b}=1$. They do not set the values for the shock parameters, since these are not necessary for their determinacy analysis. However, since I will also analyse the dynamics of the endogenous variables in response to the various shocks and their volatilities, it is necessary to set these parameters as well. As a first exercise, I assume $\rho_{A S}=\rho_{I S}=0.9$, $\rho_{F P}=\rho_{M P}=0.5$ and $\sigma_{h}=0.1$, for $h \in\{A S, I S, M P, F P\}$, i.e. the variance of all shocks are the same. ${ }^{17}$

Regarding the policy parameters, I assume that the monetary policy parameter is allowed to switch between two regimes: $\phi_{\pi, 1}=\phi_{\pi}\left(s_{t}=1\right)$ and $\phi_{\pi, 2}=\phi_{\pi}\left(s_{t}=2\right)$. Likewise, the fiscal policy parameter is allowed to switch between two regimes: $\psi_{b, 1}=\psi_{b}\left(s_{t}=1\right)$ and $\psi_{b, 2}=\psi_{b}\left(s_{t}=2\right)$. The transition matrix that defines the probabilities of the economy moving from one regime to the other is given by $P=\left[\begin{array}{cc}p_{11} & 1-p_{11} \\ 1-p_{22} & p_{22}\end{array}\right]$.

As shown by Cho \& Moreno (16), determinacy of the solution is very sensitive to the probabilities associated with each regime and the parameters of the model. This is illustrated by fixing the policy parameters in Regime $1\left(\phi_{\pi, 1}=1.5\right.$ and $\left.\psi_{b, 1}=0.05\right)$ and checking determinacy regions implied by various combinations of the parameters in Regime $2\left(\phi_{\pi, 2} \in[0,2]\right.$ and $\left.\psi_{b, 2} \in[-0.1,0.1]\right)$, and four combinations of the probabilities in matrix $P:(i)$ $p_{11}=0.99$ and $p_{22}=0.99 ;$ (ii) $p_{11}=0.99$ and $p_{22}=0.95$; (iii) $p_{11}=0.95$ and $p_{22}=0.99$ and $(i v) p_{11}=0.95$ and $p_{22}=0.95$.

Figure 1.1 shows how the determinacy regions vary with these parameters' choices. Note that the dashed lines define the quadrants of determinacy in the LRE model according to the classification in Table 1.1, with deter-

\footnotetext{
${ }^{15}$ Observe that matrix $A_{1}$ does not contain future regime-dependent parameters, thus it can be taken out of the expectations term. Matrices $R$ and $\Sigma$ are also assumed regimeindependent in this model.

${ }^{16}$ Appendix A.3 shows how the forward solution is obtained from (1-9) and (1-10) and how to check the conditions for determinacy of this solution

${ }^{17}$ Different values for these parameters were tested, but I did not make a thoughtful analysis of their impact. This is left for future work.
} 
minacy characterized by the combinations in the top right and bottom left quadrants, indeterminacy in the bottom right and no stable solution in the top left. The figure shows that the possibility of regime switching and the probabilities associated with each regime significantly alter these regions. The dark gray areas represent the pairs $\left(\phi_{\pi, 2}, \psi_{b, 2}\right)$ for which there is no stable solution for the model. The intermediate gray areas represent the $\left(\phi_{\pi, 2}, \psi_{b, 2}\right)$-pairs which imply determinate solutions. Finally, the light gray areas are those for which the pairs $\left(\phi_{\pi, 2}, \psi_{b, 2}\right)$ imply indeterminate solutions. I analysed various

Figure 1.1: Determinacy Regions
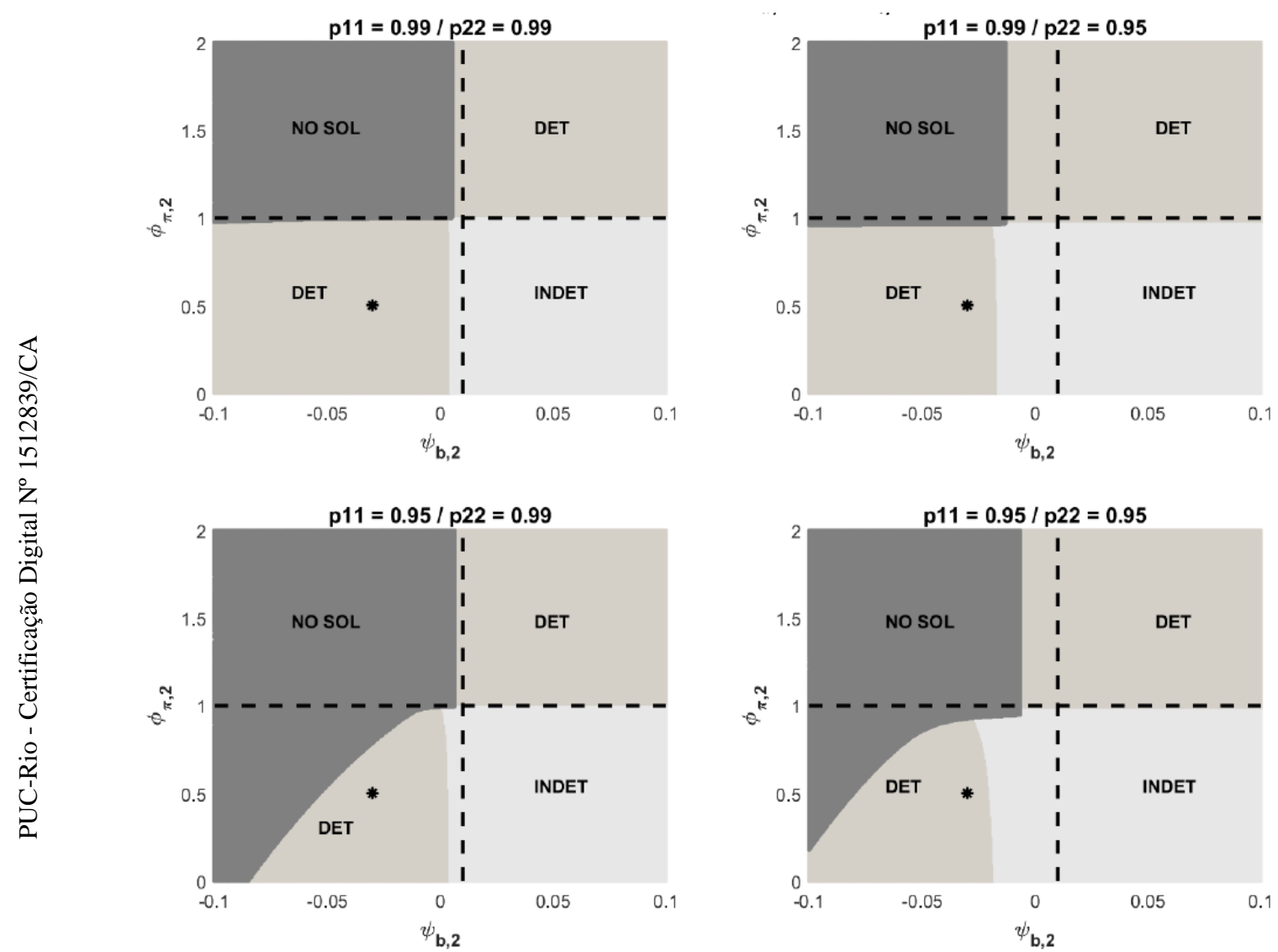

Note: This figure presents the determinacy regions given the policy parameters in the $\mathrm{AM} / \mathrm{PF}$ regime $\left(\phi_{\pi, 1}=1.5\right.$ and $\left.\psi_{b, 1}=0.05\right)$ and varying those in the $\mathrm{PM} / \mathrm{AF}$ regime $\left(\phi_{\pi, 2}\right.$ and $\left.\psi_{b, 2}\right)$ in the MSRE model. The dashed lines delimit the determinacy regions in the LRE model.

combinations with lower values for $p_{11}$ and $p_{22}$ and obtained that reducing these probabilities significantly shrinks the determiancy area in the lower left quadrant. ${ }^{18}$ Nevertheless, I want Regime 2 to be characterized by a passive monetary policy $\left(\phi_{\pi, 2}<1\right)$ and an active fiscal policy $\left(\psi_{b, 2} \leq 1-\beta\right)$, and I also want to work only with determinate solutions. The probabilities pairs depicted in Figure 1.1 are the ones for which I obtain a determinate solution

${ }^{18}$ These results are available from the author upon request. 
with the parameters chosen, both in the LRE and the MSRE models for the analysis performed next.

In the following Sections, I discuss the responses of endogenous variables to the various shocks in the model and also the impact in terms of macroeconomic volatilities of allowing for regime switches in monetary and fiscal policies.

\section{4}

\section{Impulse Responses Analysis}

In order to examine the endogenous variables' responses to each of the four shocks $\left\{z_{t}^{M P}, z_{t}^{F P}, z_{t}^{A S}, z_{t}^{I S}\right\}$, I use the impulse response functions of the LRE model, without regime switching, as a benchmark. These are obtained by solving the LRE model assuming the parameters for each regime to be constant. There are plenty of methods for solving LRE models. I apply the forward method of Cho \& Moreno (26) only to be aligned with the type of solution used for the MSRE models. Obtaining the impulse response functions for the LRE model is a straightforward task.

When turning to the analysis of impulse response functions in the MSRE models, it is important to differentiate between two cases regarding the regime behaviour along the path after the shock. In both cases, we are going to look for the expectation of each variable $k$-periods ahead, making an assumption regarding the regime observed in period $t$, when the economy is hit by a shock.

In the first case, called MSRE-free, I assume that the regime is free to change between periods $t$ and $t+k$. In this case, the regimes visited in the trajectory until $s_{t+k}=j$ are unknown. It is only known that it started at $s_{t}=i$. In the other case, called MSRE-fixed, I assume that although allowed to change, the regime observed when the shock hits the economy is kept along the path of the impulse response. This corresponds to agents having an expectation that there might be a regime change, but it does not materialize itself after the shock under analysis. Here, the regime realizations are $s_{t}=i$ in every period $t+k, k \geq 1$. Since regime switches are assumed history independent, the time spent in a certain regime does not affect the probability of switching to another regime. Both types of impulse responses can be computed recursively. Appendices A.4 and A.5 present how they are obtained.

Following Liu et. al. (15), I call expectational effects the difference between the impulse responses in the LRE model, without regime switching, and the model where switching is allowed (either in the MSRE-free case or in the MSRE-fixed case). Note that there is a subtle difference between the impulse responses obtained under the $M S R E$-fixed case and those under the LRE model. In the latter, agents completely ignore the possibility that a regime 
change might happen. In the MSRE-fixed case, agents expect regime switches to happen, but these changes do not materialize themselves along the path after the shock that is being analyzed.

Parameter combinations and probabilities associated with each regime are chosen to ensure a determinate solution. The policy parameters ${ }^{19}$ are set as follows: Regime $1(\mathrm{AM} / \mathrm{PF})-\phi_{\pi, 1}=1.5$ and $\psi_{b, 1}=0.05$ and Regime 2 $(\mathrm{PM} / \mathrm{AF})-\phi_{\pi, 2}=0.5$ and $\psi_{b, 2}=-0.03$.

The discussion focuses on the case with $p_{11}=p_{22}=0.95$, which represents less persistent regimes. However, I also comment on the results using the four combinations of $p_{11}$ and $p_{22}$ used in Figure 1.1. With these $\left(p_{11}, p_{22}\right)$ pairs and the values chosen for $\phi_{\pi, 1}, \psi_{b, 1}, \phi_{\pi, 2}, \psi_{b, 2}$, I am working in the black dot in the lower left quadrant of the plots presented in Figure 1.1, guaranteeing the solutions obtained for the MSRE model (1-2)-(1-6) are determinate. These combinations of policy parameters also guarantee determinacy in the LRE model, under both regimes.

The responses for each of the four shocks in the model are presented in Figures 1.2 to 1.5 . In these figures, red lines always correspond to Regime 1 $(\mathrm{AM} / \mathrm{PF})$, while blue lines correspond to assuming it is in Regime 2 (PM/AF). The LRE case is represented by the continuous lines, while the starred lines represent the MSRE-free case, and the dashed lines represent the MSRE-fixed case. In the figures presented in Appendix A.2, four sets of impulse responses are displayed for each of the $\left(p_{11}, p_{22}\right)$-pairs.

\subsection{1}

\section{Impulse Responses to a Monetary Policy Shock}

A well-known result in the literature of MSRE models is the emergence of expectational effects in the dynamics of macroeconomic variables when the possibility of a regime change in monetary policy is taken into account. However, as observed in Bhattarai et. al. (21), even when regime changes are not possible, the coordination between monetary and fiscal authorities has a substantial impact on the responses of the economy to shocks. They study inflation dynamics under different policy regimes and find striking differences between the responses in the $\mathrm{AM} / \mathrm{PF}$ and $\mathrm{PM} / \mathrm{AF}$ regimes. While in the $\mathrm{AM} / \mathrm{PF}$ regime inflation falls in response to a monetary policy shock, in the $\mathrm{PM} / \mathrm{AF}$ regime it increases due to the wealth effects generated by higher interest rates that increase the nominal value of debt. This is the classic FTPL mechanism, which highlights the non-ricardian characteristic of this regime.

\footnotetext{
${ }^{19}$ This choice of parameter combinations is discussed in Section 1.5, where their impacts on macroeconomic variables' volatilities are examined.
} 
If agents do not expect an increase in taxes in the future to compensate for this increase in debt, they feel wealthier now, which increases their demand for goods and services and consequently increase prices as well.

Figure 1.2 illustrates this result. In the LRE model, in response to a monetary policy shock, inflation moves in opposite directions in $\mathrm{AM} / \mathrm{PF}$ and $\mathrm{PM} / \mathrm{AF}$ regimes (red continuous line and blue continuous line, respectively). The nominal interest rate ends up increasing more in the $\mathrm{PM} / \mathrm{AF}$ regime, but because inflation increases, the real rate increases less, becoming negative after a few periods. Output decreases less on impact and gets above its steady state after a few periods. The responses of debt are very close on impact, but it increases more, and it is more persistent in the $\mathrm{AM} / \mathrm{PF}$ regime than in the $\mathrm{PM} / \mathrm{AF}$ regime. This result already highlights the importance of coordination between monetary and fiscal authorities, making it clear that the responses of one authority to shocks can lead to very different dynamics depending on what the other is doing.

Figure 1.2: IRFs to a Monetary Policy Shock
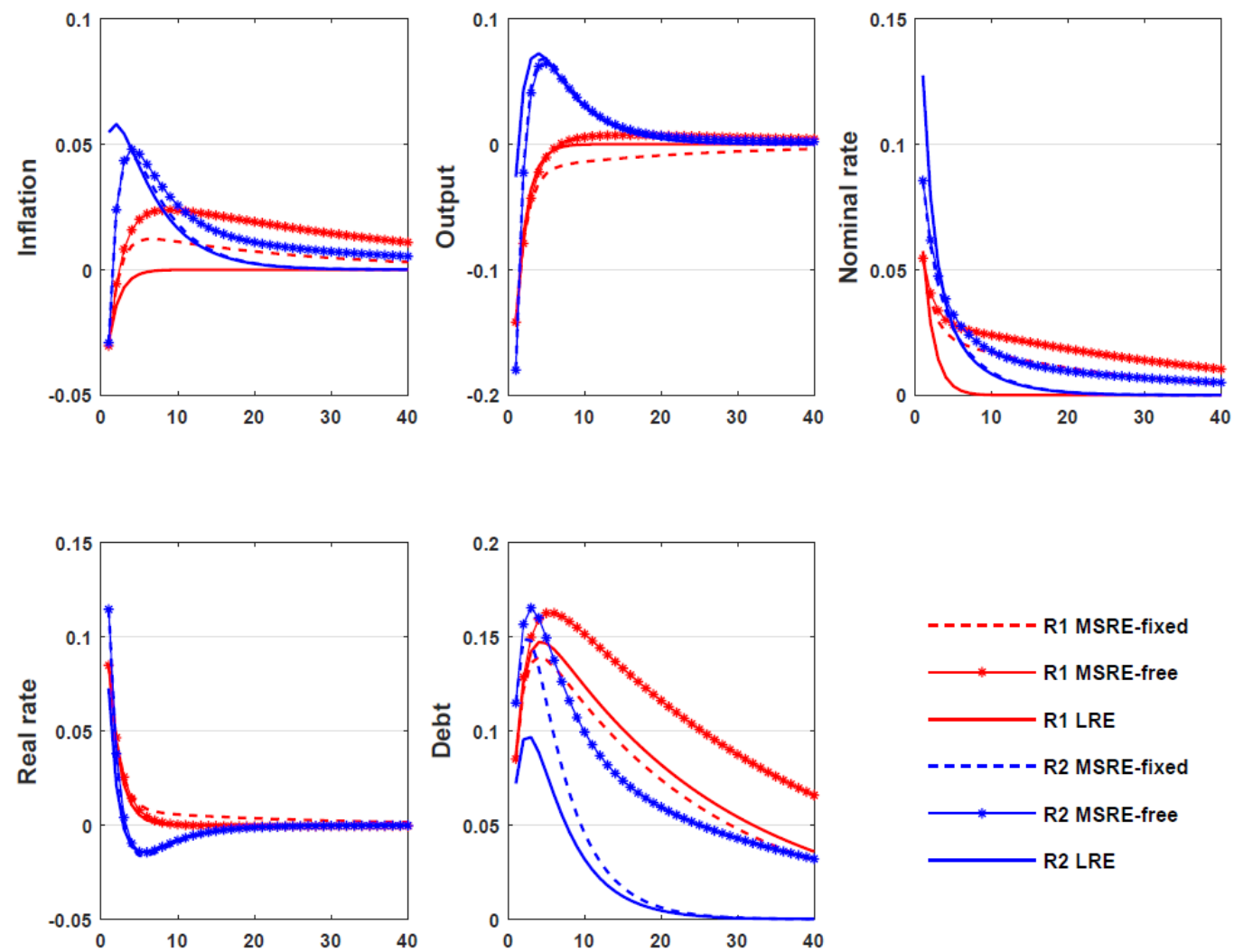

Note: This figure presents the responses to a monetary policy shock considering the probabilities $p_{11}=p_{22}=0.95$, the policy parameters in the $\mathrm{AM} / \mathrm{PF}$ regime $\left(\phi_{\pi, 1}=1.5\right.$ and $\left.\psi_{b, 1}=0.05\right)$ and in the $\mathrm{PM} / \mathrm{AF}$ regime $\left(\phi_{\pi, 2}=0.5\right.$ and $\left.\psi_{b, 2}=-0.03\right)$.

Accounting for the possibility of a regime change and consider agents' expectations regarding these changes, which are translated into the probabili- 
ties associated with each regime $\left(p_{11}, p_{22}\right)$, I obtain the expectational effects of the MSRE model.

I first examine what happens if the economy is hit by a monetary policy shock while in the AM/PF regime. Figure 1.2 shows important expectational effects on inflation, output, the nominal interest rate and the real rate, with significant changes in their dynamics. On impact, the responses continue to be close to those observed in the LRE model. But after a few periods, it is possible to see a higher persistence of the nominal interest rate either in the MSRE-free case or the MSRE-fixed case. With a higher probability of moving to a regime where fiscal policy will become active, and taxes will respond less to debt, keeping the nominal interest rate higher for a longer period generates a wealth effect, even if the regime change does not occur along the path (MSRE-fixed). Still, in this case, the increase in the nominal interest rate is more significant than that observed on inflation, so the real rate gets positive, causing the decrease in output to be more persistent than in the LRE model. When the regime is free to change along the path, the opposite happens, the nominal rate increases less than inflation and a small increase in the output gap is observed for a few periods after the shock. In the case of debt, with a high probability of moving to the $\mathrm{PM} / \mathrm{AF}$ regime $\left(p_{11}=0.95\right)$, and the regime free to change along the path, the response of debt gets more persistent, while if the regime change does not materialize itself along the path, the reaction of debt gets less persistent than in the LRE model.

Now I turn to the analysis of what happens if the economy is hit by a monetary policy shock while in the PM/AF regime. In this case, significant expectational effects are observed. The possibility of moving to the AM/PF regime alters the macro variables dynamics in striking ways, when compared with their responses when no switching is possible. The nominal interest rate increases less on impact, but with the possibility of moving to the AM/PF regime, inflation decreases on impact, with the response close to that observed under the $\mathrm{AM} / \mathrm{PF}$ regime, but it still becomes positive a few periods after the shock. When regime switching is allowed, the real rate increases more on impact, generating a larger decrease in output, though after a few periods both these variables get very close to the behaviour observed in the LRE model. The expectational effects are noticeable in the responses of debt, which responds more and is more persistent if agents expect the economy to move to the $\mathrm{AM} / \mathrm{PF}$ regime. This is the only variable for which we see clear differences between the responses in the MSRE-fixed case and the MSRE-free case. In the latter case, response of debt is more prominent and persistent than in the former. 
Appendix A.2 shows the impulse responses to a monetary policy shock for the four $\left(p_{11}, p_{22}\right)$-pairs analysed in Section 1.3.1, showing separately the responses when the economy is hit by a monetary policy shock while in the $\mathrm{AM} / \mathrm{PF}$ regime (Figure A.4) and the $\mathrm{PM} / \mathrm{AF}$ regime (Figure A.5). If in the $\mathrm{AM} / \mathrm{PF}$ regime, on impact there is no difference in the responses of the LRE model and the MSRE model. However, with less persistent regimes (decrease in $p_{11}$ and/or $p_{22}$ ), some important expectational effects are observed along the path, with the economy displaying stagflation even if the regime change does not materialize itself. If the economy starts from the PM/AF regime, expectational effects are prominent independently of the persistence of each regime, specially on impact for all variables. In the case of debt, these effects are also observed along the path. Thus, one sees that accounting for the possibility of a regime change when the economy is hit by a monetary policy shock leads to very different macroeconomic dynamics.

\subsection{2}

\section{Impulse Responses to a Fiscal Policy Shock}

The responses of endogenous variables to a fiscal policy shock change remarkably when regime switches are accounted for in the model. Figure 1.3 displays the impulse response functions of the endogenous variables to a fiscal policy shock, starting either from the AM/PF regime or the PM/AF regime, with the probabilities associated with each regime given by $\left(p_{11}=p_{22}=0.95\right)$. It shows these responses in the three cases discussed, when no regime switching is allowed (LRE model), when there is the possibility of a regime change, but the regime observed when the economy is hit by the shock does not change along the path (MSRE-fixed) and when the regime is free to change along the path (MSRE-free).

It is known that when regime switching is not allowed, and the economy is in the $\mathrm{AM} / \mathrm{PF}$ regime, inflation, output, and the nominal interest rate are determined by equations $(4.1)-(4.3)$, which form an independent block from fiscal policy. That is why these three variables are not affected by a fiscal policy shock in the LRE model under the AM/PF regime. This shock only determines the level of debt, which increases in a hump-shaped way. However, in the $\mathrm{PM} / \mathrm{AF}$ regime, as expected, debt responds much less to the fiscal policy shock and now inflation increases, but with a passive monetary policy $\left(\phi_{\pi, 2}=0.5\right)$, the nominal interest rate increases less than inflation, causing a decrease in the real rate and an expressive increase in output.

Introducing agents' beliefs that a regime change might occur has striking impacts on the dynamics of macroeconomic variables. If the economy is in 
Figure 1.3: IRFs to a Fiscal Policy Shock
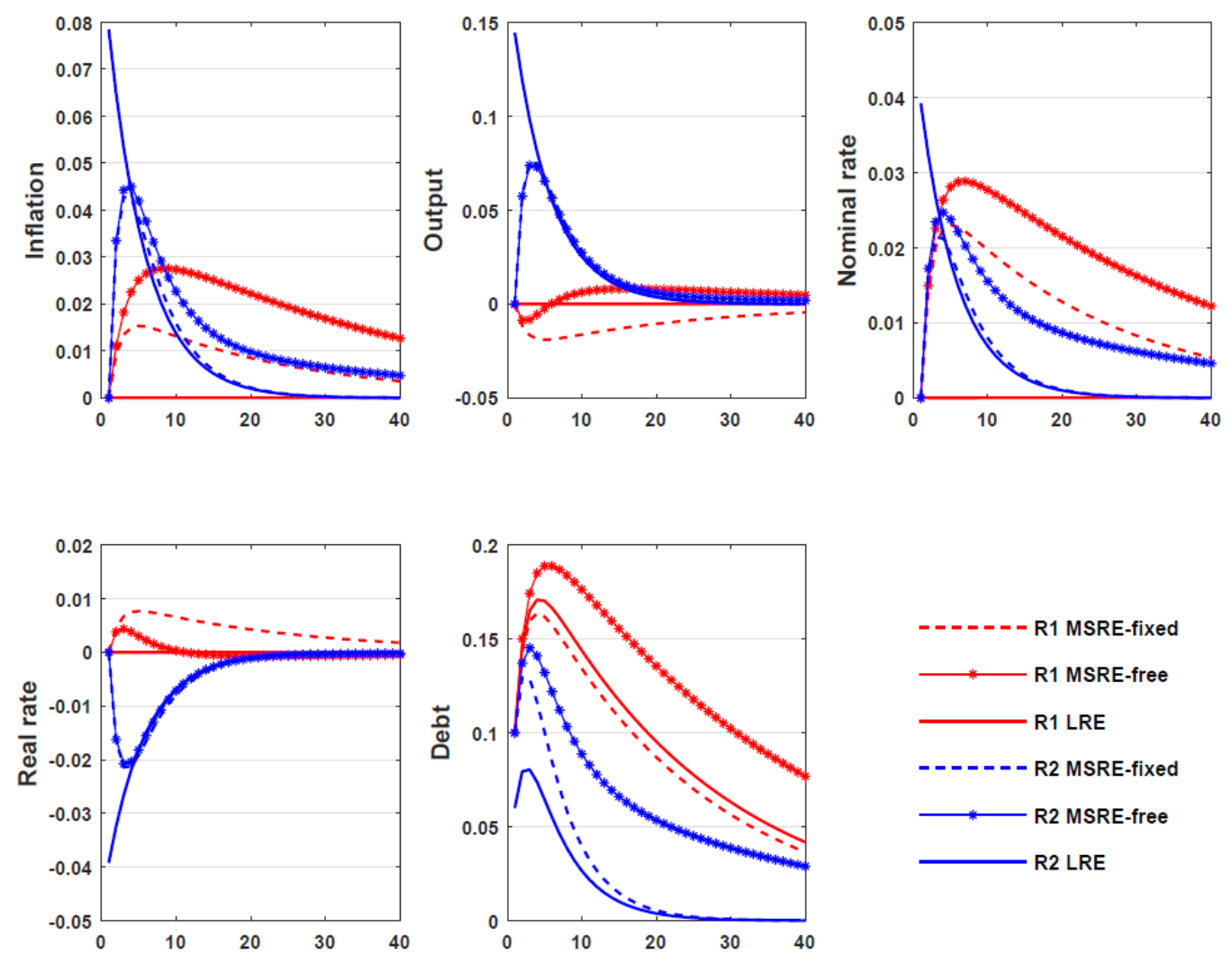

Note: This figure presents the responses to a fiscal policy shock considering the probabilities $p_{11}=p_{22}=0.95$, the policy parameters in the AM/PF regime $\left(\phi_{\pi, 1}=1.5\right.$ and $\left.\psi_{b, 1}=0.05\right)$ and in the $\mathrm{PM} / \mathrm{AF}$ regime $\left(\phi_{\pi, 2}=0.5\right.$ and $\left.\psi_{b, 2}=-0.03\right)$.

the $\mathrm{AM} / \mathrm{PF}$ regime when hit by the fiscal policy shock, expectational effects arise for all variables, both when the regime is free to change along the path (MSRE-free) and when it is kept along the path (MSRE-fixed). Under these assumptions, the possibility of moving to a $\mathrm{PM} / \mathrm{AF}$ regime causes an increase in inflation and the nominal rate, but the implications regarding the real rate are very different. When the regimes are free to move along the path, although increasing a little for a few periods after the shock, the real rate soon decreases, going below its steady-state value after a few periods. This leads to a small decrease in output right after the shock, but rapidly it increases and converges to its steady state from above.

The most remarkable result appears when agents associate some probability that there might be a regime change in the future, but it does not materialize itself for a long period, keeping the same regime along the impulse response path under analysis. In this case, inflation and the nominal interest rate increase less, but the real rate becomes positive and more persistent. This leads to a persistent decrease in output. This result is striking because merely accounting for the belief that the economy might move to a $\mathrm{PM} / \mathrm{AF}$ regime 
can cause stagflation in an $\mathrm{AM} / \mathrm{PF}$ regime, even if this regime change does not materialize itself after the shock. The shape of debt dynamics is not much affected by the possibility of a regime change when starting from the AM/PF regime. But the peak and the persistence in the $M S R E$-free case are higher than in the MSRE-fixed case. The latter is still below the response in the LRE model.

If the economy is hit by the fiscal policy shock while in the $\mathrm{PM} / \mathrm{AF}$ regime, the possibility of switching to the $\mathrm{AM} / \mathrm{PF}$ regime generates important expectational effects which alter the dynamics of macroeconomic variables. In the LRE model, inflation, nominal interest rate, and output display more prominent responses on impact and smoothly return to their steady states after a few periods. In the MSRE model, they do not respond to the shock on impact, but they increase in a hump-shaped way in the following periods. On the other hand, debt responds more than if no switching is allowed.

Appendix A.2 shows how these results are affected by the persistence of each regime, determined by the $\left(p_{11}, p_{22}\right)$-pairs. If starting from the $\mathrm{AM} / \mathrm{PF}$ regime (Figure A.6), the expectational effects are small if this regime is more persistent. When we increase th probability of moving to the PM/AF regime we see more prominent effects. On the other hand, if the economy starts from the PM/AF regime (Figure A.7), expectational effects arise for all probabilities combinations. Hence, even a small probability of moving to the AM/PF regime is sufficient to significantly change the macroeconomic dynamics.

\subsection{3}

\section{Impulse Responses to Preference and Cost Push Shocks}

In general, expectational effects arise when accounting for the possibility of regime changes after a cost-push or a preference shock, as shown in Figures 1.4 and 1.5. Even in the LRE model, there are important differences in macroeconomic dynamics if the economy is in the $\mathrm{AM} / \mathrm{PF}$ or in the $\mathrm{PM} / \mathrm{AF}$ regime when hit by the shock.

After both shocks, debt displays opposite signs, depending on the regime observed in the economy on impact. The same happens with the real rate, as a consequence of a more significant response of inflation and a smaller response of the nominal interest rate in $\mathrm{PM} / \mathrm{AF}$ regime. In this regime, both inflation and the nominal rate get negative a few periods after either a preference or a cost-push shock, which does not happen in the $\mathrm{AM} / \mathrm{PF}$ regime. Output responds more to a cost-push shock in the $\mathrm{AM} / \mathrm{PF}$ regime, while the impact of a preference shock on output is more prominent in the $\mathrm{PM} / \mathrm{AF}$ regime.

In the MSRE model, after a cost-push shock, with very persistent 
Figure 1.4: IRFs to a Cost Push Shock
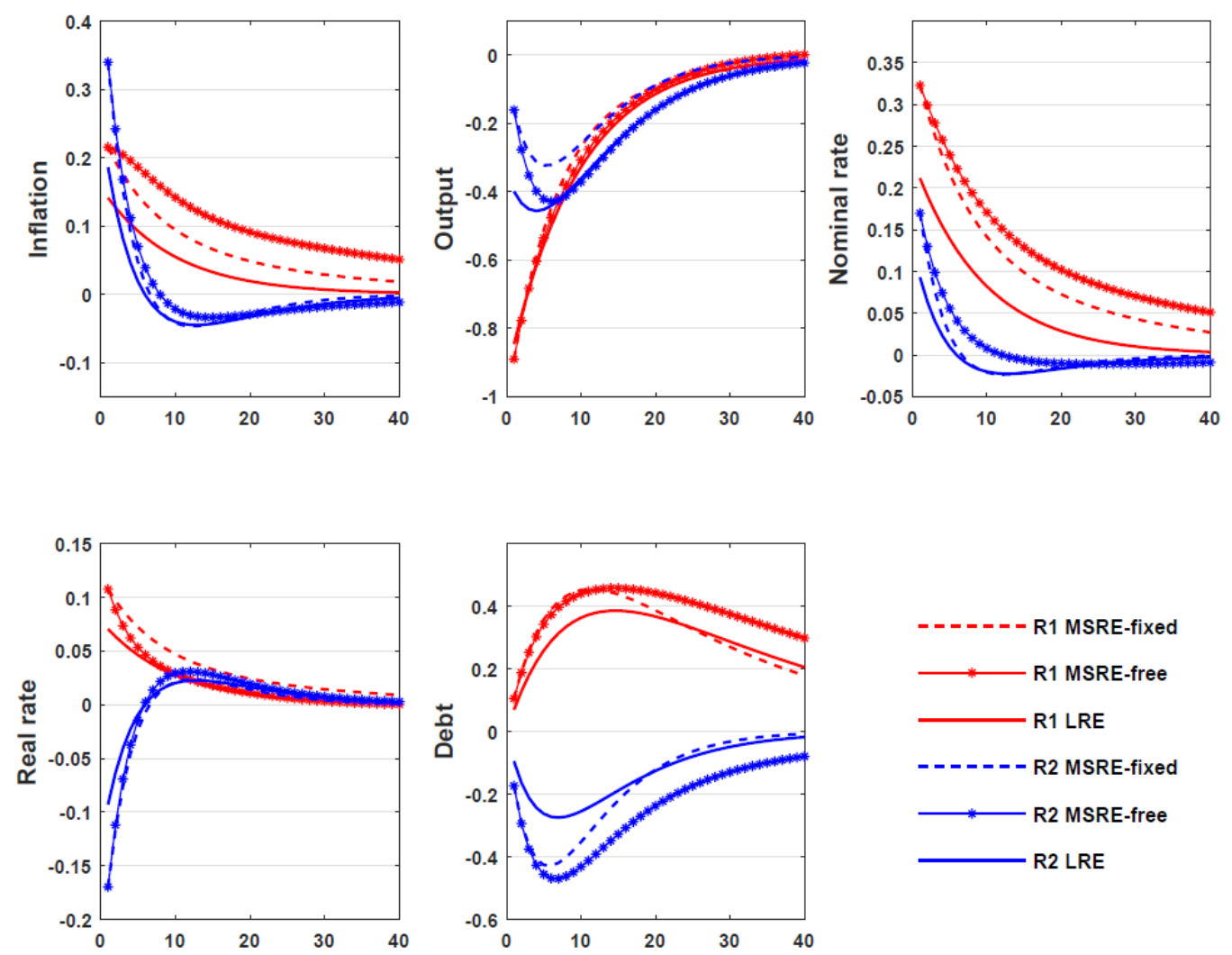

Note: This figure presents the responses to a cost-push shock considering the probabilities $p_{11}=p_{22}=0.95$, the policy parameters in the $\mathrm{AM} / \mathrm{PF}$ regime $\left(\phi_{\pi, 1}=1.5\right.$ and $\left.\psi_{b, 1}=0.05\right)$ and in the $\mathrm{PM} / \mathrm{AF}$ regime $\left(\phi_{\pi, 2}=0.5\right.$ and $\left.\psi_{b, 2}=-0.03\right)$.

regimes, expectational effects barely arise if starting from the AM/PF regime (Figure A.8). But if the economy is hit by the shock while in the PM/AF regime (Figure A.9), noticeable differences in the responses of all the variables are observed. These results are more prominent if regimes are less persistent as in Figure 1.4. Compared with the responses in the LRE model, if starting from the $\mathrm{PM} / \mathrm{AF}$ regime, inflation and the nominal interest rate increase more on impact, but with the former increasing more than the latter. This causes the real rate to decrease more in response to the shock and output to decrease much less. At the same time, accounting for regime changes amplifies the responses of debt. Starting from the AM/PF regime, allowing for regime changes amplify the responses of inflation, the nominal interest rate and debt, with the responses being slightly less persistent when the regime change does not occur along the path (MSRE-fixed).

In the case of preference shocks, expectational effects barely arise with very persistent regimes (see Figures A.10 and A.11). When the AM/PF regime gets less persistent, expectational effects amplif the responses of inflation, nominal rate, real rate and debt, especially when regime changes do not 
Figure 1.5: IRFs to a Preference Shock
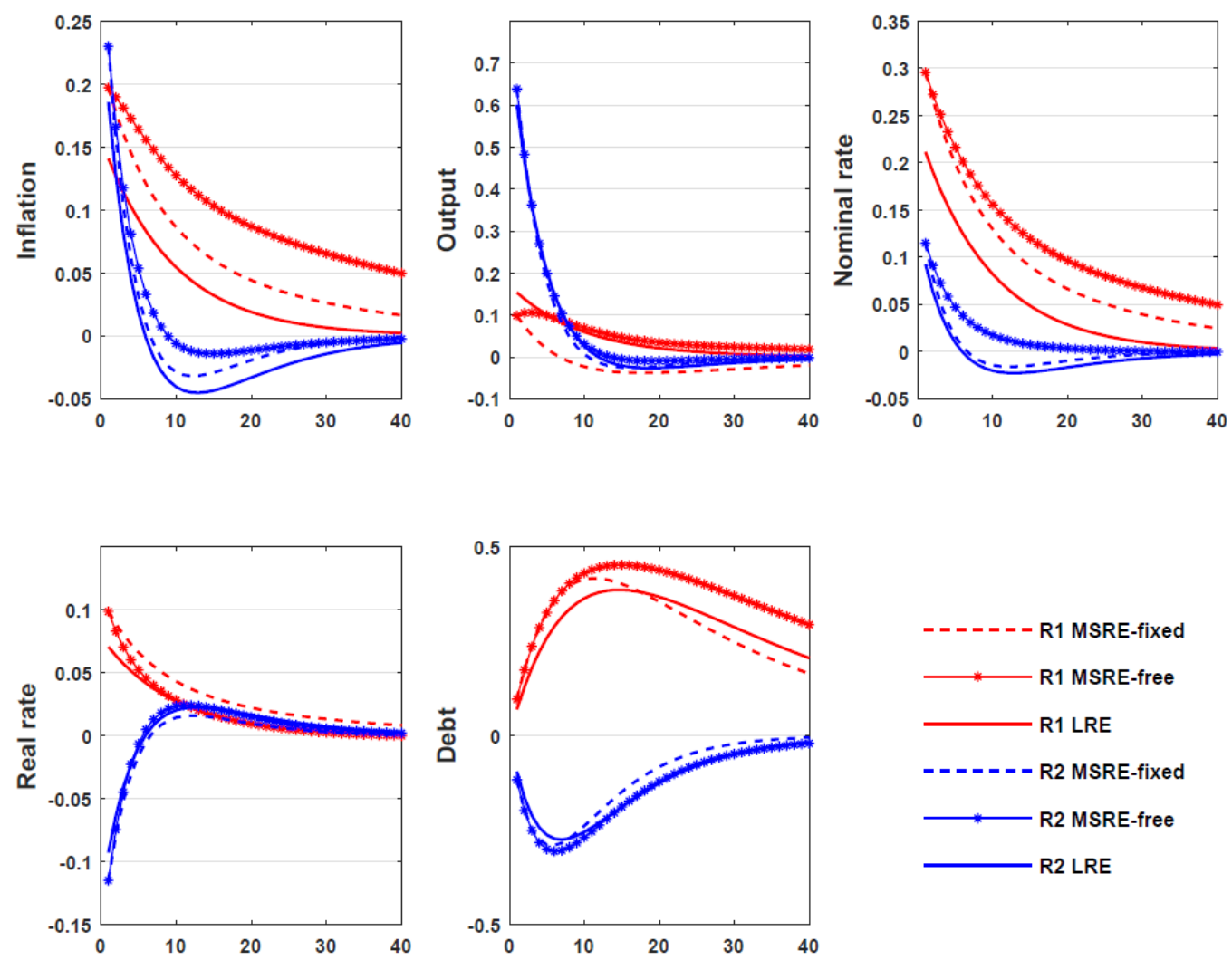

Note: This figure presents the responses to a preference shock considering the probabilities $p_{11}=p_{22}=0.95$, the policy parameters in the AM/PF regime $\left(\phi_{\pi, 1}=1.5\right.$ and $\left.\psi_{b, 1}=0.05\right)$ and in the $\mathrm{PM} / \mathrm{AF}$ regime $\left(\phi_{\pi, 2}=0.5\right.$ and $\left.\psi_{b, 2}=-0.03\right)$.

materialize themselves along the path. A exception happens for the real rate which displays a more persistent response in the MSRE-fixed case. This causes output to increase o impact, but it can become negative after a few periods. Thus, while in the $\mathrm{AM} / \mathrm{PF}$, the economy can display stagflation a few periods after it is hit by a preference shock only because agents believe there might be a chance to switch to a $\mathrm{PM} / \mathrm{AF}$ regime, even if this change does not materialize itself along the impulse response path.

\section{5}

\section{Impact of Policy Responses in the Volatility of Macroeconomic Variables}

When examining the responses of macroeconomic variables to the various shocks in Section 1.4, the parameters that characterize the stances of monetary and fiscal policies in each regime were fixed. Nevertheless, besides impacting the determinacy of the model, as seen in Figure 1.1, how the monetary authority sets the response of nominal interest rate to inflation $\left(\phi_{\pi}\left(s_{t}\right)\right)$, and the fiscal authority sets the response of taxes to debt $\left(\psi_{b}\left(s_{t}\right)\right)$, also have important effects in amplifying or undermining macroeconomic volatility. 
I use the results in Baele et. al. (23) to compute the conditional and unconditional standard deviations of macroeconomic variables in a MSRE model. Nevertheless, the application of their formulae is not straightforward in the model used here, since I assume AR(1) processes for the shocks, while they assume i.i.d. shocks, so they do not need to handle their persistence. Hence, I need to derive the standard deviations formulae in the model considering the persistence of the shocks. These are presented in Appendix A.6.

Using these results, I compute the standard deviations of inflation, output gap, nominal interest rate and debt to study how they depend on the policy parameters in the $\mathrm{AM} / \mathrm{PF}\left(\phi_{\pi, 1}, \psi_{b, 1}\right)$ and $\mathrm{PM} / \mathrm{AF}\left(\phi_{\pi, 2}, \psi_{b, 2}\right)$ regimes and the probabilities associated with each regime. Here I discuss the results assuming a low persistence of both regimes $\left(p_{11}=p_{22}=0.95\right)$, but I also examined the volatilities under the other $\left(p_{11}, p_{22}\right)$-pairs used to analyse determinacy in Figure 1.1. The results display small differences when varying these probabilities, so I discuss only one $\left(p_{11}, p_{22}\right)$-pair to illustrate them. ${ }^{20}$

Figures 1.6 to 1.9 present the results obtained. In each of these figures, I fix three out of four policy parameters and examine what happens when varying the one left. In the figures, the standard deviations of the macroeconomic variables are plotted for both $\mathrm{AM} / \mathrm{PF}$ and $\mathrm{PM} / \mathrm{AF}$ regimes (red and blues lines respectively), in the MSRE model, conditional on being in each regime (dashed lines), and in the LRE model (continuous lines). I also plot the unconditional standard deviations resulting from the MSRE model (black dotted line). The black continuous vertical line represents the value of the policy parameter used in the analysis of the impulse response functions in Section 1.4, while the black dashed vertical lines represent the value of the parameter which limits the determinacy region (determinacy frontiers), which are different from the frontiers established in the LRE model $\left(\phi_{\pi}>1\right.$ and $\left.\psi_{b}<1-\beta\right)$.

I first examine what happens when varying the degree of activeness of monetary policy in the $\mathrm{AM} / \mathrm{PF}$ regime $\left(\phi_{\pi, 1}\right)$, which is shown in Figure 1.6. In the LRE model, this parameter only affects the variables' volatilities in the AM/PF regime. A more active monetary policy (higher $\phi_{\pi, 1}$ ) decreases the volatility of inflation and nominal interest rate, while increasing the volatilities of output and debt.

When the possibility of regime changes is considered, conditional on being in the $\mathrm{AM} / \mathrm{PF}$ regime, volatilities get amplified in general. Differently from what is observed in the LRE model, now the volatilities of the endogenous variables, conditional on being in the $\mathrm{PM} / \mathrm{AF}$ regime, also depend on how active

\footnotetext{
${ }^{20}$ The complete set of results for the four $\left(p_{11}, p_{22}\right)$-pairs are available from the author upon request.
} 
Figure 1.6: Standard deviations as a function of $\phi_{\pi, 1}$
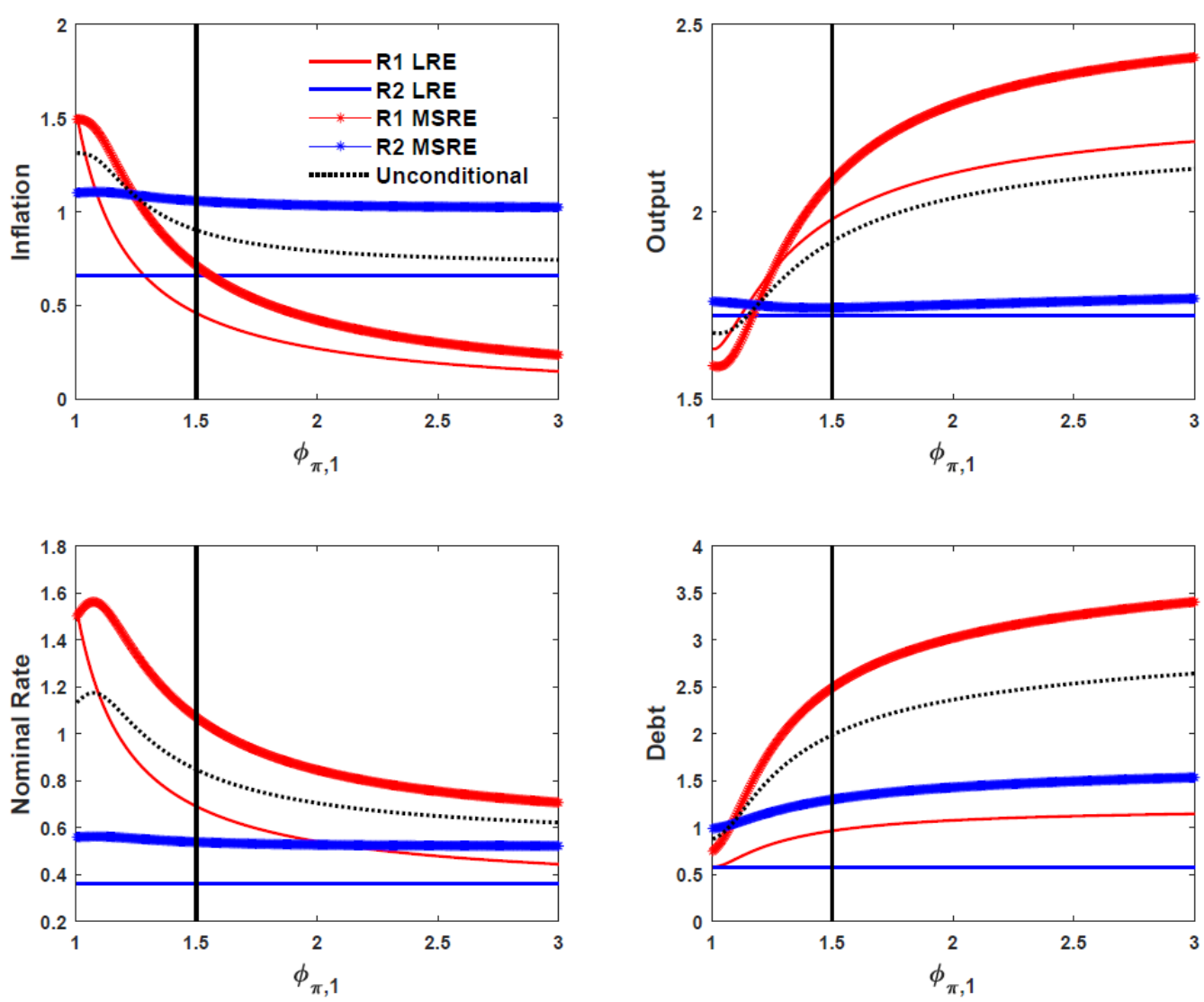

Note: This figure presents the standard deviations of the endogenous variables as a function of the monetary policy parameter in the $\mathrm{AM} / \mathrm{PF}$ regime $\left(\phi_{\pi, 1}\right)$, fixing the other policy parameters $\left(\psi_{b, 1}=0.05 / \phi_{\pi, 2}=0.5 / \psi_{b, 2}=-0.03\right)$, and considering the probabilities $p_{11}=p_{22}=0.95$. The black vertical line represents $\phi_{\pi, 1}=1.5$, which is the value assumed for this parameter in the analysis of the impulse response functions in Section 1.4.

monetary policy is in the $\mathrm{AM} / \mathrm{PF}$ regime. The volatilities of all endogenous variables increase. It is worth highlighting how the unconditional volatilities of these variables also depend on how active monetary policy is supposed to be. Note that, in general, when analysing the impulse response functions in Section 1.4, the value set for the monetary policy parameter in the AM/PF regime $\left(\phi_{\pi, 1}=1.5\right)$ does not represent the peak of the variables' volatilities.

Looking at the degree of passiveness of fiscal policy in the AM/PF regime $\left(\psi_{b, 1}\right)$, Figure 1.7 shows that when it becomes more passive (higher $\psi_{b, 1}$ ), it does not affect macro variables' volatilities under either regime if switching is not allowed, with the exception of debt in the $\mathrm{AM} / \mathrm{PF}$ regime, which is naturally the only variable that responds to variations in $\psi_{b, 1}$ in the LRE model. Nevertheless, in the MSRE model the volatilities of inflation, nominal rate and debt are higher, conditioned on either regime, and they are decreasing on $\psi_{b, 1}$. Only output in the PM/AF regime can get less volatile than in the LRE model, depending on how passive fiscal policy is. Note that with less 
persistent regimes, there is a lower bound that limits the reduction of the fiscal policy parameter after which the MSRE model is no longer determined. When the fiscal policy parameter gets less passive (lower $\psi_{b, 1}$ ), approaching this determinacy frontier, volatilities get even higher. Thus, a more passive fiscal policy in the $\mathrm{AM} / \mathrm{PF}$ regime tends to reduce overall volatility in the economy if regimes are allowed to change frequently. Note that the value picked for this parameter in Section $1.4\left(\psi_{b, 1}=0.05\right)$ is not in the range of peak standard deviations.

Figure 1.7: Standard deviations as a function of $\psi_{b, 1}$
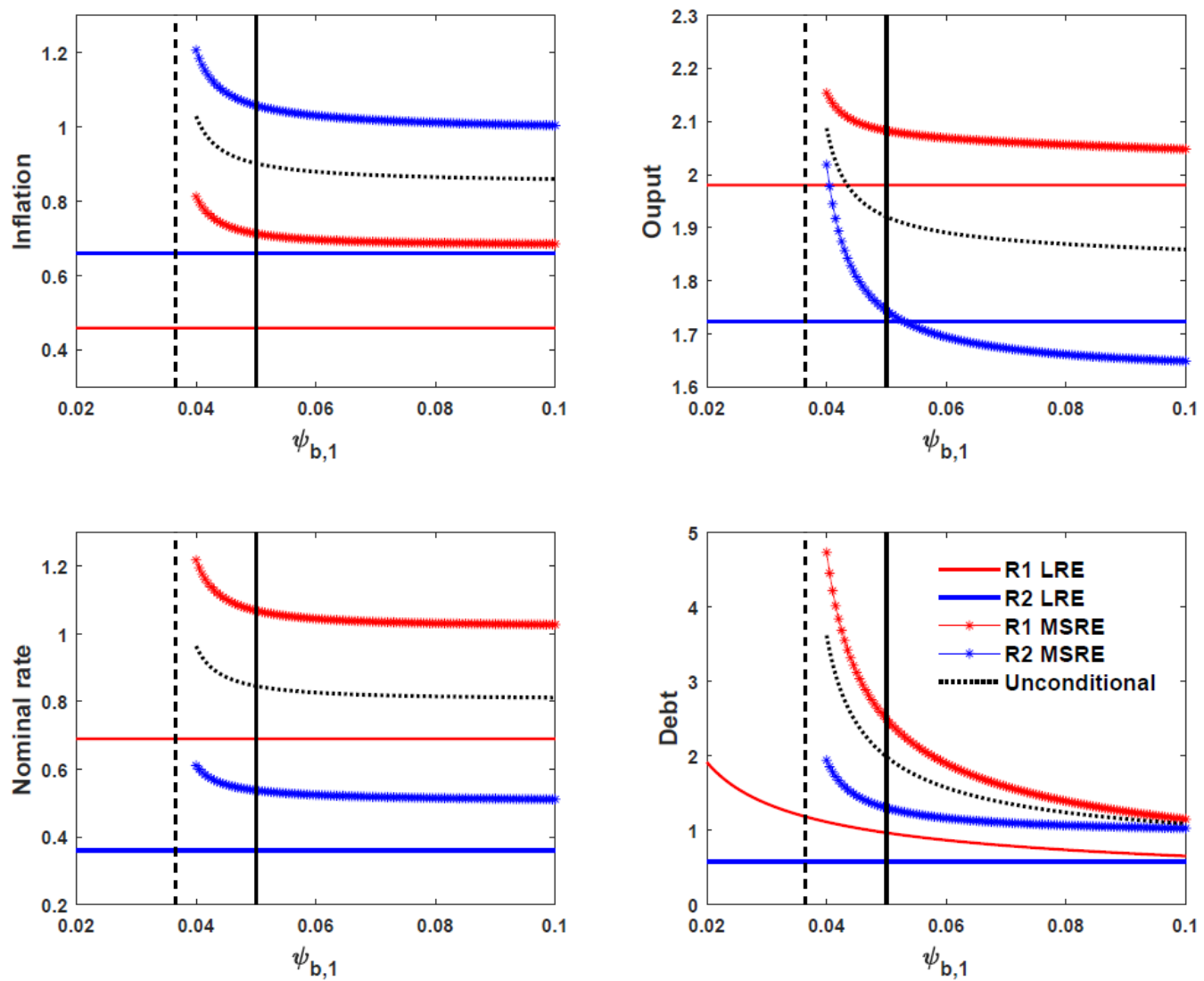

Note: This figure presents the standard deviations of the endogenous variables as a function of the fiscal policy parameter in the $\mathrm{AM} / \mathrm{PF}$ regime $\left(\psi_{b, 1}\right)$, fixing the other policy parameters $\left(\phi_{\pi, 1}=1.5 / \phi_{\pi, 2}=0.5 / \psi_{b, 2}=-0.03\right)$, and considering the probabilities $p_{11}=p_{22}=0.95$. The black vertical line represents $\psi_{b, 1}=0.05$, which is the value assumed for this parameter in the analysis of the impulse response functions in Section 1.4. The black dashed vertical line represents the determinacy frontier. For values of $\psi_{b, 1}$ below this value, the solution is no longer determinate.

Striking differences in the volatilities of exogenous variables are observed when varying the policy parameters in the $\mathrm{PM} / \mathrm{AF}$ regime. As shown in Figure1.8, a less passive monetary policy (higher $\phi_{\pi, 2}$ ) increases the volatilities of the nominal interest rate and inflation in the $\mathrm{PM} / \mathrm{AF}$ regime, even when no switching is allowed (LR model). As $\phi_{\pi, 2}$ starts approaching the value of the 
determinacy frontier (which for the LRE model is equal to 1), the volatilities of inflation and nominal interest rate increase exponentially, while slowly decreasing for output and debt. When considering the possibility of a regime change, the conditional and unconditional volatilities of all macroeconomic variables are amplified, specially when $\phi_{\pi, 2}$ increases, getting closer to the determinacy frontier. This is particularly true for the volatility conditional on being in the $\mathrm{AM} / \mathrm{PF}$ regime, which does not respon to changes in $\phi_{\pi, 2}$ in the LRE model. Note that the value chosen to examine the impulse responses in Section $1.4\left(\phi_{\pi, 2}=0.5\right)$ does not represent areas with peak volatilities.

Figure 1.8: Standard deviations as a function of $\phi_{\pi, 2}$
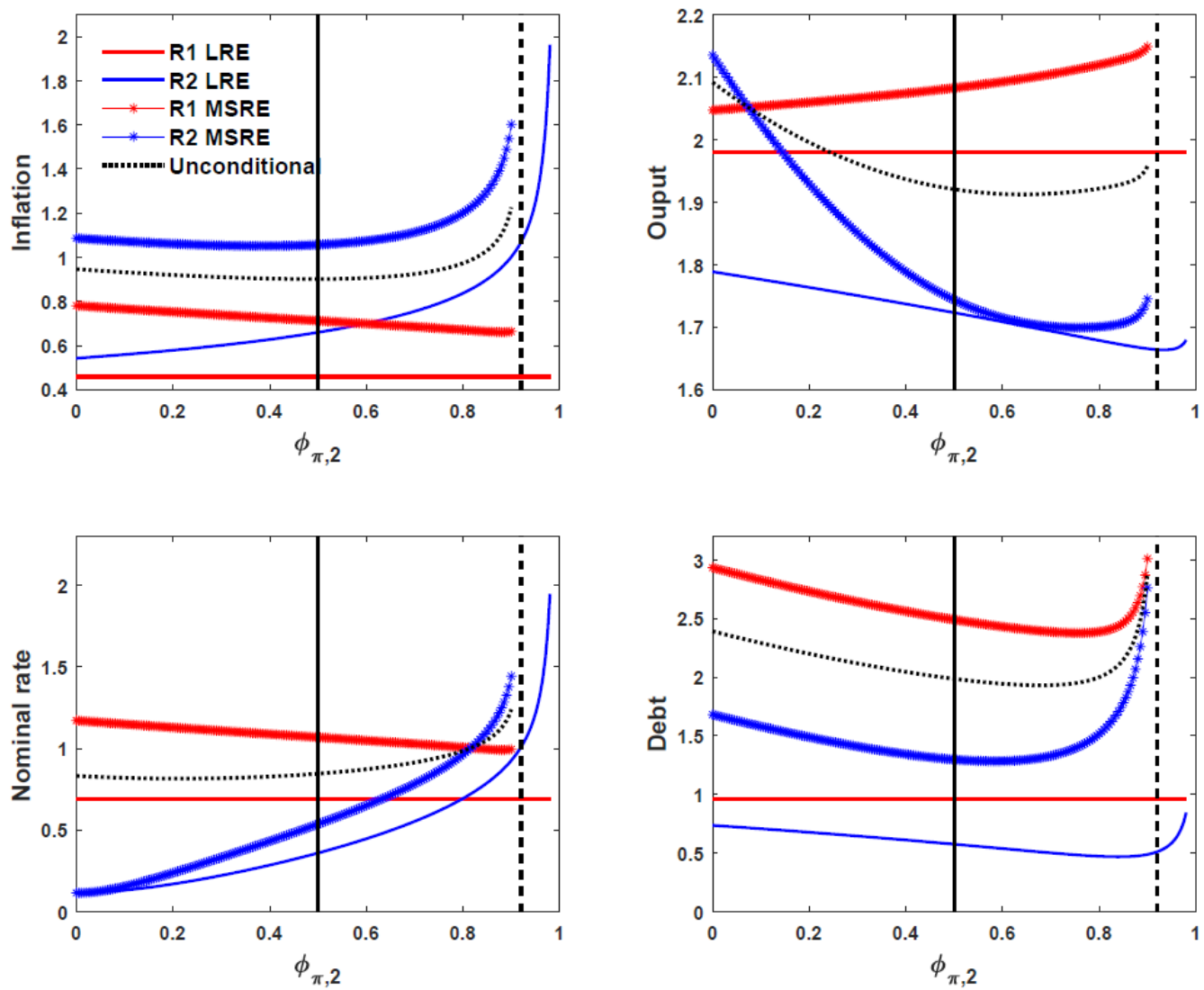

Note: This figure presents the standard deviations of the endogenous variables as a function of the monetary policy parameter in the $\mathrm{PM} / \mathrm{AF}$ regime $\left(\phi_{\pi, 2}\right)$, fixing the other policy parameters $\left(\phi_{\pi, 1}=1.5 / \psi_{b, 1}=0.05 / \psi_{b, 2}=-0.03\right)$, and considering the probabilities $p_{11}=p_{22}=0.95$. The black vertical line represents $\phi_{\pi, 2}=0.5$, which is the value assumed for this parameter in the analysis of the impulse response functions in Section 1.4. The black dashed vertical line represents the determinacy frontier. For values of $\phi_{\pi, 2}$ above this value, the solution is no longer determinate.

Figure 1.9 shows the impact of a more active fiscal policy in the PM/AF regime (lower $\psi_{b, 2}$ ). Here it is possible to observe remarkable differences in the volatilities implied by the LRE and MSRE models. Considering the possibility of a regime change amplifies the volatilities for all variables in both regimes. 
As we saw in Figure 1.1, decreasing the persistence of each regime shrinks the determinacy regions when varying $\psi_{b, 2}$, imposing new determinacy frontiers for the MSRE model. With the value chosen to analyze the impulse responses in Section $1.4\left(\psi_{b, 2}=-0.03\right)$ we are not in the area of extreme volatilities. To sum up, it gets clear from this analysis that accounting for the possibility

Figure 1.9: Standard deviations as a function of $\psi_{b, 2}$
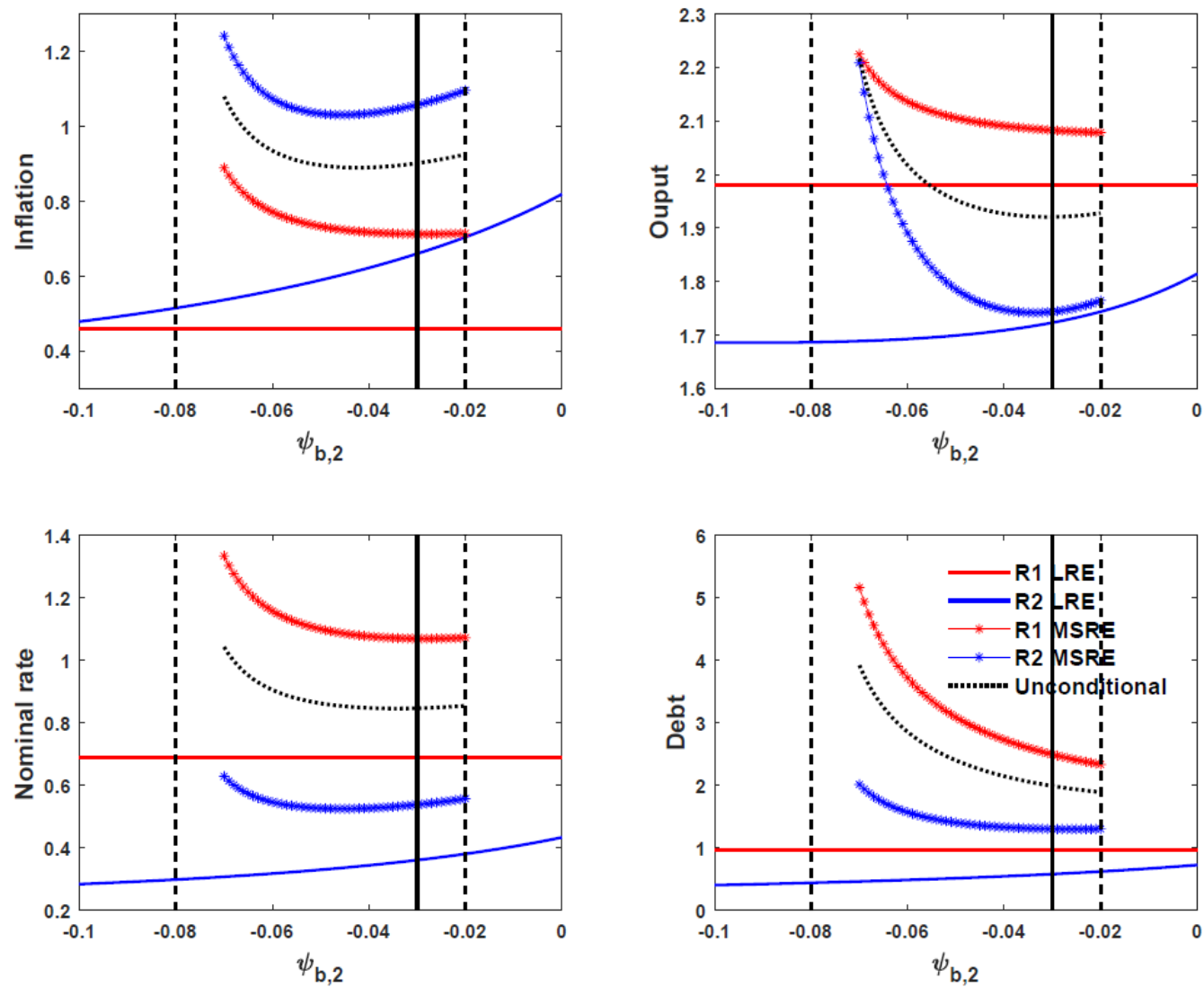

Note: This figure presents the standard deviations of the endogenous variables as a function of the fiscal policy parameter in the $\mathrm{PM} / \mathrm{AF}$ regime $\left(\psi_{b, 2}\right)$, fixing the other policy parameters $\left(\phi_{\pi, 1}=1.5 / \psi_{b, 1}=0.05 / \phi_{\pi, 2}=0.5\right)$, and considering the probabilities $p_{11}=p_{22}=0.95$. The black vertical line represents $\psi_{b, 2}=-0.03$, which is the value assumed for this parameter in the analysis of the impulse response functions in Section 1.4. The black dashed vertical line represents the determinacy frontier. For values of $\phi_{\pi, 2}$ above this value, the solution is no longer determinate.

of regime switches have important effects on the volatility of macroeconomic variables. Besides, the degree of activeness or passiveness of both monetary and fiscal policies, and the probabilities associated with each regime, are also crucial in determining these volatilities. 


\section{6}

\section{Conclusion}

Using a simple model, I obtained interesting results when accounting for agents' expectations that regime switches in monetary and fiscal policy stances might happen in the future. The responses of the endogenous variables to the various shocks that hit the economy can differ, even when this change does not materialize itself. The possibility of regime switches also has important effects in the volatilities of macroeconomic variables. These results make it clear that not only the coordination between monetary and fiscal authorities is important in determining macroeconomic dynamics, but also how these policies are communicated to the public and translated into their beliefs regarding the authorities' future behaviour.

The results highlight not only the importance of coordination between monetary and fiscal authorities in responding to the various shocks that hit the economy, but also how crucial it is to consider the effect of agents' expectations on the outcomes of the adopted policies. In an environment with increasing uncertainty, besides evaluating the measures to be taken, it is also key to correctly communicate and signal them to the public.

When the fiscal scenario starts to pose risks for the conduction of monetary policy, it is important that both authorities show to be aware of the fiscal deterioration. The fiscal authority needs to take the necessary measures to correct the route, while the monetary authority needs to conduct its policy making clear that there are fiscal requirements for it to work normally and deliver inflation at the target.

Although giving very interesting insights of the role of expectations in affecting macroeconomic dynamics and volatility, this work leaves a long avenue for future research. A first issue to be raised is that one could imagine that agents are able to learn from the history of regimes observed. After not observing a regime change that was being expected, agents could review their expectations and this would certainly affect the dynamics and volatilities obtained.

Another limitation of our work is that we assume probabilities associated with each regime to be fixed and analyse different values for them. Nevertheless, it would be interesting to analyse a model where a shock could trigger a change in these probabilities. One could assume that strong institutions would imply in smaller probabilities of regime changes.

An additional extension would be to work with an open economy model in order to be able to analyse the effects of these regime changes in the exchange rates and how it would interact with the other variables. 


\section{2 \\ Fiscal Stimulus at the Zero Lower Bound: The Role of Expectations and Policy Coordination}

\section{1 \\ Introduction}

The significant amount of stimulus provided by fiscal authorities since the burst of the 2007-2008 financial crisis brought the discussion about the impact of these policies to the center of the economics debate. ${ }^{1}$ Knowledge about the magnitude of the multipliers, which express how much output changes in response to a given fiscal measure, is relevant to guide the choice among the available policy instruments (taxes, transfers or spending). This knowledge is a crucial input for deciding the size and the duration of the stimulus that will be implemented. For the conduct of monetary policy, one must also understand the impact of fiscal policies on output and inflation and evaluate how monetary authority's actions might contribute to amplifying or undermining this impact.

There is a general understanding that fiscal multipliers cannot be established as an absolute number under all circumstances and across countries. In fact, the discussion about them has converged to the characterization of their determinants. Corsetti et. al. (28) point out that the specificities of each country, and the economic environment, largely affect the impact fiscal measures have on the real economy. Analysing the characteristics of DSGE models that are relevant to fiscal multipliers, Coenen et. al. (29) observe that, besides depending on the structural parameters of the model, a series of factors are essential to explain such remarkably dispersed estimates. Among the most relevant, they highlight the duration of the stimulus, the degree of monetary policy accommodation, the fiscal instruments used and their operating channels.

The way monetary policy responds to the implementation of fiscal policies is a critical aspect that one should consider when studying the multipliers. Following an economic stimulus through increases in government spending,

${ }^{1}$ According to the IMF Fiscal Monitor, in the US, government spending as a fraction of GDP increased by $2.1 \%$ on average in the period 2006-2014, reaching its historical peak in 2009, when the Congress passed the American Recovery and Reinvestment Act. In the same period, gross debt to GDP ratio increased by 41.3 b.p.. Most advanced economies observed these increases and the peak in 2009. 
a higher degree of monetary accommodation implies larger multipliers. The extreme case is when, during a deep recession, monetary policy reaches its limit, with the nominal interest rate hitting the zero lower bound. In this circumstance, as shown by Christiano et. al. (30), fiscal policy ends up being more stimulative than in normal times. Eggertsson (31) studies the effects of a fiscal stimulus at zero interest rates using a standard New Keynesian model. His results point out that the impact of government spending increases is more substantial in this environment, while cuts in labour or capital taxes can be recessive.

In general, the debate about the interaction between fiscal and monetary policies is based on the analysis of their actions during the crisis. Nonetheless, there has been some discussion regarding the role played by expectations about future fiscal and monetary policy measures. Using a DSGE model in which policy rules may evolve according to a probability distribution, Davig \& Leeper (32) show that the multipliers dependent not only on the monetary-fiscal regime during the period the stimulus is provided, but also on the regime expected in future periods. Woodford (33) points that "careful signalling about the likely direction of future policy is likely to be as important as current actions." Eggertsson \& Woodford (34) analysed the optimal conduct of monetary and fiscal policies in this environment. They highlight the role played by expectations regarding future policy in mitigating the distortions created by the zero lower bound.

I extend the work of Eggertsson (31) to study the impact on economic activity of agents' expectations regarding policies when the crisis is over and the zero lower bound is no longer binding. Eggertsson (31) studies fiscal policy in a two-state model where monetary and fiscal policies revert to their precrisis values when the shock goes back to the steady state. This work extends his framework by allowing for a transitional state in which the shock that led to the zero lower bound is no longer affecting the economy, but policies can be expected to deviate credibly from their steady-state values.

This Chapter revisits the main results of optimal policies under a liquidity trap which supports that stimulus should stay in place for a longer horizon than the duration of the shock. Indeed, optimal monetary policy under commitment, when there are adverse shocks to the natural rate of interest, requires keeping the interest rate at the zero-lower bound, even when the shock is no longer causing it to bind, to generate inflationary expectations at the exit. I ask whether fiscal policy can directly help to boost the economy by also committing to keep the stimulus once the crisis is over. The inclusion of a transitional state allows analysing several combinations of practical interest in which fiscal policy 
is expansionary for a longer horizon, while the monetary authority may or may not be accommodative, even when the zero lower bound is no longer binding.

On the fiscal side, I discuss the effect of stimuli that are expected to last more than the crisis spell, or that might be carried out with a delay. On the monetary side, I examine the effect of keeping the interest rate at zero for a few periods after the crisis is over.

I use the model set-up in Eggertsson (31), which gives rise to an aggregate demand/supply sort of analysis. Although simple, this model allows obtaining useful analytical results which highlight that considering only shortrun multipliers can be misleading and that expectations regarding future policies play a crucial role in determining the levels of output and inflation during the crisis state. This work contributes by extending the discussion about government spending multipliers, but also regarding the impact of stimulus implemented through income taxes.

The main result of the Chapter is that monetary and fiscal policies, to be more expansionary, should last longer than the duration of the shock and be coordinated in this expansionary move. This coordination is required not only during the crisis, but also regarding the commitment to future policies. It is crucial to the fiscal authority to be able to respond quickly to the shock, minimizing implementation delays and correctly signalling the duration of the stimulus.

In general, the combinations of policies that reduce the falls in output and inflation during the crisis the most are those in which the fiscal stimulus is carried out with minimum delay during the crisis. Besides, keeping the stimulus when the shock is no longer causing the zero lower bound to bind, and agents expect its association with an accommodative monetary policy, can create expectations of higher inflation when the crisis is over and, even if small decreases in output are expected, attenuate the depth of the crisis.

It is interesting to point out that, as shown in Eggertsson (31), an increase in income taxes stimulates the economy when the zero lower bound is binding, although its impact is smaller than if providing the stimulus through increases in government spending. Nonetheless, in contrast to what we observe with government spending, keeping the stimulus after the crisis is over can improve the economy, even without monetary policy accommodation. Hence, a mistake in the time of ending the stimulus provided through increases in income taxes is not as harmful as the one in the timing of reverting an increase in government spending. This difference is also valid for the implementation of policies. Delaying the increase in taxes until the shock is no longer causing the zero lower bound to bind can still reduce the depth of the crisis. In this case, it 
is better than not implementing any policy at all. In contrast, if the stimulus is carried out through increases in government spending that are expected to occur only when the crisis is over, without any accommodation from monetary policy, it is better not to implement any policy at all.

I discuss these various policy combinations in the analysis that follows. The next Section situates this Chapter on the recent literature about fiscal multipliers, especially considering their values when the zero lower bound for the nominal interest rate is binding. Section 2.3 introduces the setup of the model and discusses the available policy responses to the crisis under the extension of a two-state to a three-state economy, allowing for the occurrence of a transitional state. The analytical solutions for output and inflation in each state are derived in Section 2.4. Section 2.5 performs an analysis of the multipliers in a calibrated model, while Section 2.6 discusses the impact of different policies' combinations on output and inflation in each state. Section 2.7 concludes the Chapter.

\section{2}

\section{Related Literature}

The discussion about the size of fiscal multipliers has been intense and led to a vast literature on the topic. I summarize here a few papers more closely related to the analysis presented in this work. ${ }^{2}$ The focus is on the debate regarding the response of output and inflation to fiscal stimuli during a crisis caused by a shock that makes the nominal interest rate hit the zero lower bound. It is known that a combination of many factors is responsible for determining the impact of fiscal measures in this environment. Ramey \& Zubairy (37) point to theoretical evidence that characteristics such as the persistence of spending changes, how they are financed, how monetary policy reacts and the tightness of labour markets can significantly affect the magnitude of multipliers.

In a structural vector autoregression (VAR) analysis with data from groups of both advanced and emerging countries, Ilzetzki et. al. (38) obtain that the degree of openness of the economy, the exchange rate regime and the monetary policy reaction are the most influential factors explaining the broad dispersion of estimates for the multipliers across countries. Their estimations suggest larger multipliers in more closed economies (fewer leakages of aggregate demand towards imports), in open economies with fixed exchange rate regimes and when the monetary policy is more accommodative.

${ }^{2}$ For an overview of this discussion see Hall (35) and Spilimbergo et. al. (36). 
The extreme case of accommodative monetary policy is when the nominal interest rate hits the zero lower bound and the monetary authority can no longer stimulate the economy by decreasing it. In such an environment, with the economy facing deep reductions in both output and inflation, there is plenty of evidence that fiscal stimulus is effective in reducing the depth of the recession.

Christiano et. al. (30) show that spending multipliers are more than three times larger when the nominal interest rate is zero, compared with the situation of positive interest rates. They emphasize that the larger the fraction of government spending that occurs while the nominal interest rate is zero, the larger the value of the multiplier. Woodford (33) also analyses variations in government spending. In his set-up, the multiplier at the zero lower bound is monotonically increasing in the expected duration of the crisis.

Eggertsson (31) studies this issue in a set-up similar to Woodford's, but with a richer set of fiscal instruments. He shows that if private agents expect government spending to be higher in all states in which the lower bound is binding, the expected contraction is reduced, giving an incentive for them to spend more. Government's commitment to keeping higher spending as long as the zero lower bound is still binding is critical for this result. A surprising result that labour tax cuts can be recessive follows. In normal times, reductions in labour taxes would make workers willing to increase labour supply, leading to a more abundant supply of goods and, thus, downward pressures on prices. In response, the monetary authority reduces the nominal interest rate and output goes up. When the zero lower bound binds, the same tax cuts still create deflationary pressures, making agents expect lower prices in all states while it is still binding. But the central bank cannot react by further decreasing the nominal interest rate. So the real interest rate goes up, implying a downward movement of output. ${ }^{3}$

All these authors highlight the importance of implementation timing for fiscal measures to have maximum effect in fighting the recession. The expectation that the stimulus might continue after the crisis is over, while monetary policy goes back to its usual stance, can undermine the positive effect obtained during the collapse. Woodford (33) studies the possible occurrence of a transitional state generated by the continuation of higher government spending after the recession is over. This work corroborates his results. However, in the case of continuous fiscal expansion, the multiplier ends up being smaller than if the policy was perfectly timed to end as soon as the crisis

${ }^{3}$ This is an application of the Paradox of Toil which says that, once the nominal interest rate hits the zero lower bound, if everybody tries to work more, there will be less work in the aggregate (see Eggertsson (39)). 
is over. The longer agents expect the spending stimulus to last, the smaller is the multiplier. Ultimately, it can even become negative. Eggertsson (31) finds similar results.

In the extreme case, if the increase in government spending is expected to be permanent, meaning that it will be above its steady-state level in the long run, it will imply higher output and lower inflation in the long term. The expectation of lower inflation in the future undermines the effect of higher government spending in the short run and, for some calibrations, it can be contractionary even during the crisis. This effect explains the contradictory results obtained by Cogan et. al. (40). Using a model similar to Christiano et. al.(30), but assuming government spending to increase permanently during the crisis, they obtain significantly smaller multipliers.

In the opposite direction, Corsetti et. al. (41) discuss the need of many countries to face significant retrenchment in government spending and analyse the effects of such anticipated spending reversals. They show that the beneficial effect of public expenditure is quite sensitive to when the reversal starts. An early and intense reversal may lower fiscal multipliers and extend the zero lower bound episode.

Eggertsson (31) also analyses how expectations about future monetary policy can affect the macroeconomic aggregates during the crisis. He assumes the central bank credibly commits to a higher inflation target in the future. Expectations of higher inflation in the future decrease real interest rate in the crisis state, stimulating spending during the recession. This policy, however, requires a high degree of credibility for the central bank to make such trustworthy announcements.

Eggertsson \& Woordford (34) point that in a liquidity trap, this type of commitment would be optimal. In their set-up, they show that keeping the nominal interest rate at the lower bound for a few quarters after the natural rate of interest has returned to its normal level, will cause the economy to display an output boom and an increase in inflation. The authors highlight that "a credible commitment to behave in this way, after the zero bound has ceased to bind, dramatically reduces the price and output declines that occur during the period when the central bank is constrained by the zero bound."

Denes et. al. (42) extend the work of Eggertsson (31) by explicitly considering the government budget constraint to analyse the consequences to debt dynamics of policies adopted when the zero lower bound is binding. They show that austerity measures (cutting government spending and/or increasing taxes) may increase, rather than decrease the short-run deficit. They also examine how the deficit created during the crisis affects expectations and, 
consequently, short-run demand. These expectations refer to how long-run taxes and spending will be adjusted to bring down debt to its pre-crisis level. However, a fundamental assumption in their work is that monetary policy sets the nominal interest rate so that inflation is zero when the zero lower bound is not binding.

The studies mentioned above use a set-up similar to the one adopted in the present study, analysing the effects of expectations about future policies. Nevertheless, they do not put together an extensive analysis of possible policy combinations like the one shown here. They investigate forward guidance considering monetary policy or government spending in isolation, but do not study the combination of both policies when the crisis is over, besides only examining government spending as the fiscal instrument.

\section{3}

\section{The Model}

I follow the setup of a simple New Keynesian model presented in Eggertsson (31). His paper provides the detailed microfoundations of the nonlinear model. The model is linearised around the long-run steady state and summarized through an $(I S)$ equation, an aggregate supply $(A S)$ relation and the definition of a monetary policy rule $(M P)$. These three equations are the starting point for our analysis.

The $(I S)$ equation comes from the households' optimal decisions, using the aggregate resource constraint to substitute out for consumption. Its linearised version is given by

$$
\text { (IS) } \hat{Y}_{t}=E_{t} \hat{Y}_{t+1}-\sigma\left(i_{t}-E_{t} \pi_{t+1}-r_{t}^{e}\right)+\left(\hat{G}_{t}-E_{t} \hat{G}_{t+1}\right),
$$

where $i_{t}$ is the one-period risk-free nominal interest rate, $\pi_{t+1}$ is the inflation rate, $\hat{Y}_{t} \equiv \log \left(\frac{Y_{t}}{\bar{Y}}\right)$ is the deviation of output from its steady-state value and $\hat{G}_{t} \equiv\left(\frac{G_{t}-\bar{G}}{\bar{Y}}\right)$ the deviation of government spending $\left(G_{t}\right)$ from its steady-state value $(\bar{G})$, as a fraction of steady-state output $(\bar{Y}) \cdot r_{t}^{e}$ is an exogenous shock defined as $r_{t}^{e} \equiv \log \beta^{-1}+E_{t}\left(\hat{\xi}_{t}-\hat{\xi}_{t+1}\right)$, where $\hat{\xi}_{t} \equiv \log \xi_{t} / \bar{\xi}$ is the deviation of a consumer's preference shock from its steady state. $\beta>0$ is the discount factor and $\sigma>0$ is the intertemporal elasticity of substitution of private expenditure.

A critical assumption is that government spending is not a perfect substitute for private consumption. As pointed out by Eggertsson (31), this is the type of expenditure which is effective in increasing demand (infrastructure or military spending are examples). If they were substitutes, cuts in private spending would offset an increase in government spending, and aggregate spending would not change. 
The aggregate supply relation $(A S)$ is derived from the firms' optimal decisions and is given by

$$
(A S) \quad \pi_{t}=\kappa \hat{Y}_{t}+\kappa \psi\left(\chi^{I} \hat{\tau}_{t}^{I}-\sigma^{-1} \hat{G}_{t}\right)+\beta E_{t} \pi_{t+1},
$$

where $\hat{\tau}_{t}^{I}=\tau_{t}^{I}-\bar{\tau}^{I}$ is the percentage increase in income tax rate, ${ }^{4,5}$ while $\chi^{I}>0, \kappa>0, \omega>0$ and $\psi>0$ are model parameters. ${ }^{6}$

Finally, the monetary policy rule is as follows:

$$
(M P) \quad i_{t}=\max \left(0, r_{t}^{e}+\phi_{\pi} \pi_{t}+\phi_{y} \hat{Y}_{t}\right)
$$

where it is assumed that $\phi_{\pi}>1$ and $\phi_{y}>0$. This specification rules out negative values for the nominal interest rate.

The $(I S)$ equation, together with the monetary policy rule, defines the aggregate demand $(A D)$ relation in this model. Equilibrium will be the solution of the system formed by the aggregate demand and supply equations. Eggertsson (31) shows that, given a path for $\left\{\hat{G}_{t}, \hat{\tau}_{t}^{I}\right\}$ determined by fiscal policy, and an exogenous path for $\left\{r_{t}^{e}\right\}$, an equilibrium is a collection of stochastic processes for output, inflation and the nominal interest rate $\left\{\hat{Y}_{t}, \pi_{t}, i_{t}\right\}$ that solves the system of equations (2-1) - (2-3).

Another critical assumption is that Ricardian equivalence holds. This means that lump-sum transfers in period $t$ or in future periods offset temporary variations in either $\hat{\tau}_{t}^{I}$ or $\hat{G}_{t}$. In the absence of this hypothesis, Denes et. al. (42) point out that how a fiscal expansion is financed, through adjustments in future taxes or spending, can have significant effects on short-run demand. This implies that a plan about how short-run budget deficits or surpluses will be met in the future should complement a given government stimulus provided when the zero lower bound is binding. Although important, I will not discuss the impact of the fiscal stimulus on debt generated by the absence of lump-

${ }^{4}$ We follow Denes et. al. (42) who model taxes $\left(\tau_{t}^{I}\right)$ levied on income from both labour and the households' claims on firms profits. They explain that if only wages were taxed, there would be a disproportionate fall in tax revenues in a recession. This would exaggerate the results and rely too much in the complete wage flexibility in the model. With this more conservative assumption, income tax is proportional to output. Any fall in real wages will be reflected by an increase in profits, and taxing wages and profits at the same rate means we abstract from this redistribution aspect of the model.

${ }^{5}$ Regarding other types of taxes discussed in Eggertsson (31), he shows that the effects of sales taxes represent a negative scale of those caused by variations in $\hat{G}_{t}$. So it would be redundant to analyse them here. For the sake of space, the analysis of taxes levied on capital income is left for future work.

${ }^{6}$ These parameters depend on the baseline parameters of the model as follows: $\chi^{I} \equiv \frac{1}{1-\bar{\tau}^{I}}$, $\omega \equiv \frac{\bar{v}_{h h} \bar{H}}{\bar{v}_{h}}$, where $\bar{v}_{h}$ and $\bar{v}_{h h}$ are, respectively, the first and second derivatives of the utility function with respect to labour, and $\bar{H}$ is the level of labour in steady state. $\psi \equiv \frac{1}{\sigma^{-1}+\omega}$ and $\kappa \equiv \frac{(1-\alpha)(1-\alpha \beta)}{\alpha} \frac{\sigma^{-1}+\omega}{1+\omega \theta}$, where $0<\alpha<1$ is the fraction of firms whose prices stay unchanged in each period and $\theta>1$ is the elasticity of substitution among differentiated goods. 
sum taxes closing the government budget constraint. This would significantly increase the number of policy combinations to be analysed and negatively affect the tractability of the paper.

Although quite simple, the model has the advantage of providing closedform solutions and allowing the discussion of the effects of policies during the zero lower bound episode. In what follows I analyse a set of possible short-run allocations in response to the exogenous shock $r_{t}^{e}$. Depending on the strength of the shock and the policy responses to it, two scenarios are possible. I call "normal times" the scenario where the shock is not large enough to make the nominal interest rate hit the zero lower bound. The other scenario, called the "crisis state," occurs when the shock causes the nominal interest rate implied by rule (2-3) to reach the zero lower bound. ${ }^{7}$ The fall in output and inflation caused by the shock varies a lot, depending not only on the policy actions taken during the crisis state (short run) but also on expectations about the implementation of policy actions after the crisis is over. I analyse how possible combinations of present and future policies impact crisis-state allocations.

First, I summarize the two-state economy case presented by Eggertsson (31). Then I show the set-up of the three-state economy that includes a transitional state. The next Section displays the derivation of the allocations in each state.

\subsection{1}

\section{Revisiting the Literature - Two-state economy}

The analysis in Eggertsson (31) starts by assuming that at time $t=T_{0}$ the economy is hit by a shock that causes $r_{t}^{e}$ to go to $r_{S}^{e}<0$. The return of the shock to the steady state at time $t=T_{\text {exit }}$ is exogenously given. In his basic set-up, there are two possible states for this economy. The short run, or crisis state, $\left(t \in\left[T_{0}, T_{\text {exit }}\right)\right)$, characterized by the period in which $r_{t}^{e}$ goes below its steady-state value $\left(r_{t}^{e}=r_{S}^{e}\right)$. And the long term $\left(t>T_{\text {exit }}\right)$, when $r_{t}^{e}$ returns to the steady state $\left(r_{t}^{e}=\bar{r}\right)$. Woordford (33) interprets this shock as a severe disruption in financial intermediation that causes a spike in credit spreads, thus decreasing $r_{t}^{e}$. Eggertsson (31) explains it as a preference shock that lowers $\xi_{t}$ because suddenly everyone wants to save more, so the real interest rate must decline for output to stay constant. I assume that the evolution of this shock is independent of either monetary or fiscal policy actions. Therefore, measures to stimulate the economy do not revert the shock but can attenuate the recession it generates.

${ }^{7}$ As in Eggertsson \& Woodford (34) and Woodford (33), Eggertsson (31) assumes that even when the shock is large enough to cause the zero bound to bind, local approximations to both the model structural relations and the welfare objective are still accurate. 
If the shock is large enough, it can make the zero lower bound on the nominal interest rate a binding constraint in policy rule (2-3). In this scenario, the monetary authority can no longer reduce the nominal interest rate further to stimulate the economy, so the government can only rely on a combination of the available fiscal instruments $\left(\hat{G}_{t}, \hat{\tau}_{t}^{I}\right)$ to mitigate the recession caused by the fall in $r_{t}^{e}$.

Assuming that the shock causes the nominal interest rate to go to zero $\left(i_{t}=0\right)$, Figure 2.1 illustrates the two-state economy with a crisis state. In the short run, the shock causes output and inflation to go below their steady-state values $\left(Y_{S}, \pi_{S}\right)$. This allocation depends on the size of the shock $\left(r_{S}^{e}\right)$ and the fiscal measures adopted $\left(G_{S}, \tau_{S}^{I}\right)$. In each period $t \in\left[T_{0}, T_{\text {exit }}\right)$, after the shock hits the economy, there is an exogenous probability $\mu$ that the shock will still be at $r_{S}^{e}$ in period $t+1$. With probability $(1-\mu)$ it will return to $\bar{r}$ in period $t+1$ and the economy goes back to the steady state. I assume that once back to the steady state, $r_{t}$ stays at $\bar{r}$ thereafter. Eggertsson (31) (Proposition 2 Page 70) shows that, in the long run, with $\hat{G}_{t}=\hat{\tau}_{t}^{I}=0$, there is a locally unique bounded solution to the system (2-1) - (2-3) such that $\hat{Y}_{t}=\pi_{t}=0$ and $i_{t}=\bar{r}, \forall t>T_{\text {exit }}$.

Figure 2.1: Benchmark Case - Two-state economy

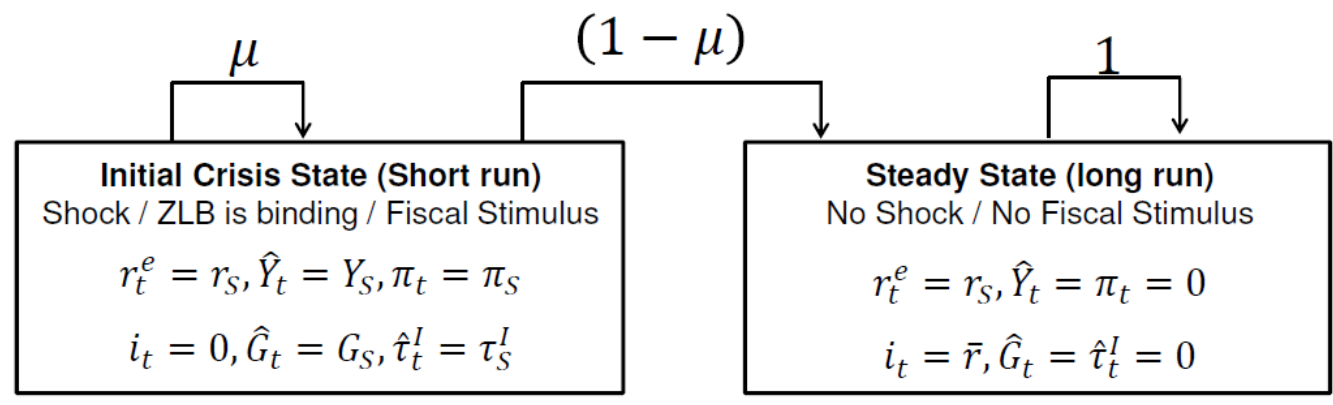

Eggertsson (31) highlights as a general principle in this class of models that, when the zero lower bound is binding, successful policy actions are those which do not aim at increasing aggregate supply, but increasing the aggregate level of spending in the economy. This happens because, with zero nominal interest rates, output is demand determined. This does not mean that aggregate supply is irrelevant since it is crucial to pin down expectations about future inflation. It is important to note that in this environment, incentives to aggregate supply are counter-productive since they can create deflationary expectations. He precisely points out that "policy should not be aimed at increasing the supply of goods when the problem is that there are not enough buyers." 


\subsection{2}

\section{The Three-State Economy}

A critical assumption of significant part of the analysis in Eggertsson (31) is that the implemented stimulus policy is perfectly correlated with the shock. This means that government spending and/or income taxes will deviate from their steady-state values as long as $r_{t}^{e}=r_{S}^{e}$, i.e. while the crisis lasts. Once $r_{t}^{e}$ goes back to $\bar{r}$, fiscal instruments also return to their long-run levels $\left(\hat{G}_{t}=\hat{\tau}_{t}^{I}=0, \forall t>T_{\text {exit }}\right)$.

I extend the analysis in Eggertsson (31) by allowing the government to credibly commit to implementing a combination of monetary and fiscal policies, even after the recession is over. During the crisis, expectations about future values of output and inflation play an important role in determining the depth of the collapse. Eggertsson \& Woodford (34) point out that it makes a crucial difference if the government can commit to creating inflation in the future. They show that it is optimal to maintain a loose monetary policy for a few quarters after the crisis is over. Assuming VAT taxes, they also show it is optimal to increase them during the crisis and reduce them when the zero lower bound is no longer binding.

Expectations regarding future actions from both monetary and fiscal authorities affect the levels of output and inflation during the crisis. It is important to note from equations (2-1) and (2-2) that, besides the direct impact of future government spending on short-run aggregate demand through the term $E_{t} \hat{G}_{t+1}$, any future policy actions that affect output and inflation after the crisis is over, will also have effects on short-run aggregate demand and supply relations through the terms $E_{t} \hat{Y}_{t+1}$ and $E_{t} \pi_{t+1}$.

The goal is to understand these effects and show how the impact of stimulus actions during the crisis depends on expectations about future interactions between these policies. On the monetary policy side, I want to model the expectation that the central bank might keep the interest rate at a fixed value $i_{M}$ (which can be zero) for a few periods ${ }^{8}$, even after $r_{t}^{e}$ returns to $\bar{r}$. On the fiscal policy side, I analyse the impact of the termination timing of the stimulus provided during the short run and of implementation delays. I examine these policies adopted in coordination, but also cases where each authority acts separately.

Figure 2.2 illustrates this three-state economy set-up. As before, the idea is that at time $t=T_{0}$, the economy is hit by a shock $r_{t}^{e}=r_{S}^{e}$, the zero lower

${ }^{8}$ It will be shown that this cannot be a permanent policy as it would lead to solution indeterminacy. In Chapter 3, I discuss what an optimal level for this transitional state interest rate would be, depending on the expected duration of this state. 
bound binds $\left(i_{t}=0\right)$, the fiscal authority uses a combination of its instruments $\left(G_{S}, \tau_{S}^{I}\right)$ to undermine the crisis' effects, but output and inflation still stay below their steady-state levels $\left(Y_{S}, \pi_{S}\right)$. This state lasts until an uncertain period $t=T_{\text {exit }}$, when $r_{t}^{e}$ returns to $\bar{r}$. In every period of the recession $t \in$ $\left[T_{0}, T_{\text {exit }}\right)$, there is probability $\mu$ that the economy stays in the crisis state in the following period. With probability $(1-\mu) b$ it goes to a transitional state, while with probability $(1-\mu)(1-b)$ it jumps straight to the long run. ${ }^{9}$

Figure 2.2: Transitional State Case - Three-state economy

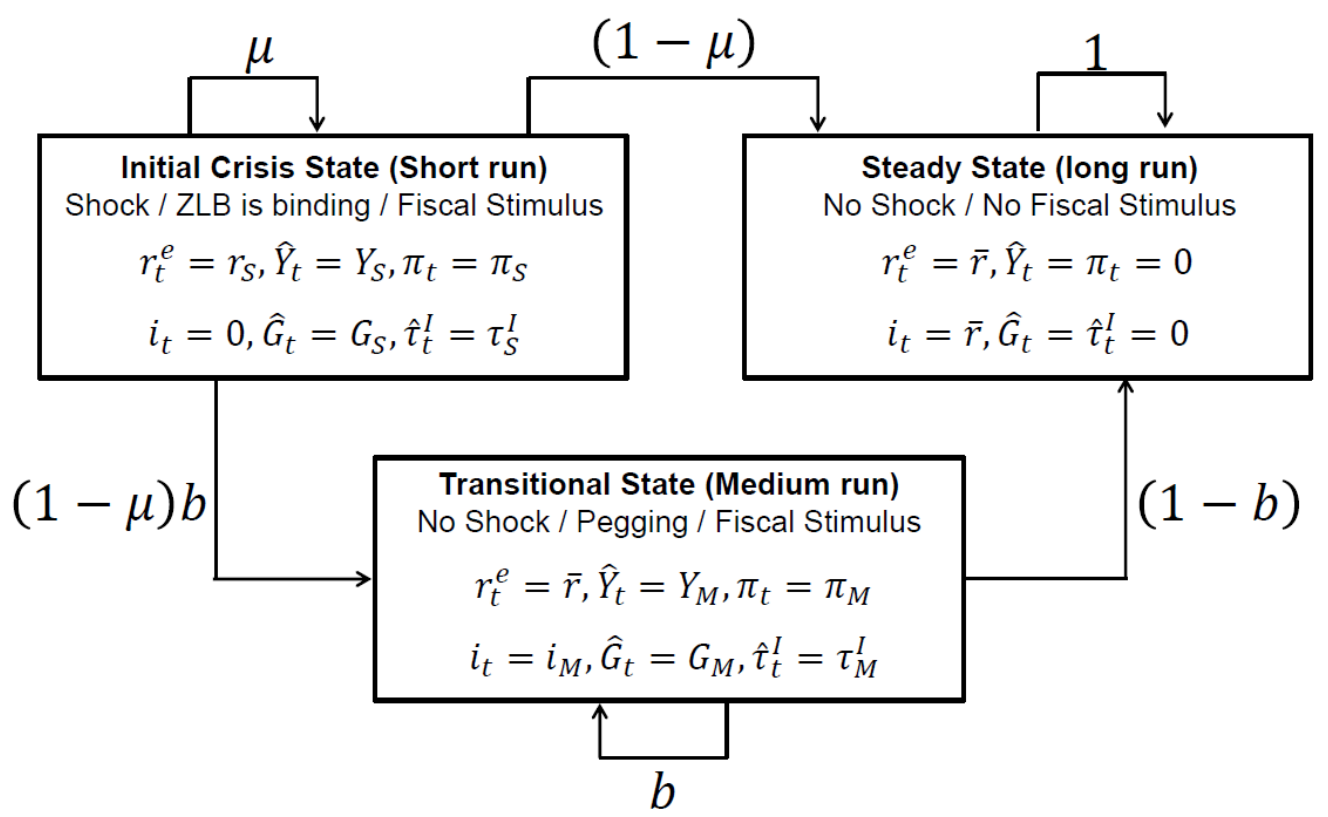

A transitional state occurs because agents associate some probability that after exiting the crisis state, the authorities might still deviate from their steady-state policies. They might expect that the monetary authority will deviate from the rule (2-3), keeping a fully accommodative monetary policy for a few periods after the crisis is over, maintaining nominal interest at $i_{M}=0$. Agents can also expect the fiscal stimulus implemented during the crisis not to be perfectly correlated with it, lasting for a few periods after the zero lower bound is no longer binding $\left(G_{M}, \tau_{M}^{I}\right)$. Expectations may be that only one or both these policies will be used in the transitional state. This creates a medium-run allocation, with output and inflation deviating from their steadystate levels $\left(Y_{M}, \pi_{M}\right)$, that lasts until an exogenously given period $t=T_{M}$. In each period $t \in\left[T_{\text {exit }}, T_{M}\right)$, there is probability $b$ that the economy stays in the transitional state in $t+1$, while with probability $(1-b)$ it goes to the long run.

${ }^{9}$ Throughout the analysis, both probabilities $\mu$ and $b$ are assumed independent from monetary and fiscal policy actions and from each other. 
With a temporary fiscal stimulus (during the crisis state or lasting until the transitional state only), in the long run, monetary and fiscal policies go back to their steady-state plans $\left(i_{L}=\bar{r}, G_{L}=\tau_{L}^{I}=0\right)$, returning output and inflation to their steady-state levels as well $\left(Y_{L}=\pi_{L}=0\right)$. Once the economy goes to the long run, I assume that it stays at this level thereafter.

The proposal is to analyse a way the monetary authority has to create expectations of higher inflation in the future, by assuming that the central bank can credibly commit to keeping the nominal interest rate fixed for a few periods, even after $r_{t}^{e}$ returns to its steady-state value at $\bar{r}$. I study the possibility that the fiscal authority can also signal that it will keep using a combination of its policy instruments when the zero lower bound is no longer binding, which might be associated with a future accommodative monetary policy or not. ${ }^{10}$

Table 2.1 summarizes the policy choices available for monetary and fiscal authorities in each state. In the short run, the monetary authority cannot stimulate the economy further, since the zero lower bound is binding, leaving the fiscal authority as the only one able to provide stimulus. It may pick one of its instruments, either using government spending or income taxes. I assume that once chosen in the short run, the same instrument is used throughout the states (this rules out, for example, using government spending in the short run and income taxes in the medium run or vice-versa). ${ }^{11}$

Table 2.1: Summary of possible policy choices

\begin{tabular}{|c|c|c|}
\hline & Monetary Policy & Fiscal Policy \\
\hline $\begin{array}{l}\text { Short run } \\
\left(r_{t}^{e}=r_{S}^{e}\right)\end{array}$ & $Z L B$ is binding $\left(i_{S}=0\right)$ & $\begin{aligned}(i) & \text { None } \\
(\text { ii }) & \hat{G}_{S} \\
(\text { iii }) & \hat{\tau}_{S}^{I}\end{aligned}$ \\
\hline $\begin{array}{l}\text { Medium run } \\
\qquad\left(r_{t}^{e}=\bar{r}\right)\end{array}$ & $\begin{aligned}(i) & i_{M}=0 \\
(\text { ii) } & i_{M}=\bar{r}+\phi_{\pi} \pi_{M}+\phi_{y} Y_{M}\end{aligned}$ & $\begin{aligned}(i) & \text { None } \\
(i i) & \hat{G}_{M} \\
(\text { iii }) & \hat{\tau}_{M}^{I}\end{aligned}$ \\
\hline
\end{tabular}

In the medium run, the fiscal authority can choose to return the instru-

${ }^{10}$ Eggertsson (31) models the promise of future inflation with a monetary policy rule committed to a higher inflation target. He shows that this not only increases inflation expectations for all periods after $r_{t}^{e}$ returns to $\bar{r}$, but also reduces the fall in short-run inflation in all periods when the zero lower bound still binds. He does not analyse an association of this policy with future fiscal actions though.

${ }^{11}$ I will not assume different levels for the fiscal instruments in each state. When used both in the crisis and transitional states, we will have $G_{S}=G_{M}$ or $\tau_{S}^{I}=\tau_{M}^{I}$. The subscripts for the respective states are kept to allow identifying where the impacts on the levels of output and inflation are coming from. 
ment used in the short term to its steady state, or it can keep the stimulus for a few periods after the crisis is over. On the other hand, the monetary authority will choose from two possible policies: $(i)$ keep the nominal interest rate at $i_{M}=0$ for a few periods after the crisis is over; ( $\left.i i\right)$ return to policy rule (2-3) as soon as the crisis is over. I assume that if the fiscal stimulus is extended to the transitional state, associated with an accommodative monetary policy, than these policies are perfectly correlated, i.e., they go to the long run at the same time $t=T_{M}$.

The next Section presents solutions for output and inflation in each state, under different hypothesis regarding expectations about monetary and fiscal policies, after the crisis is over.

\section{4}

\section{Solution Allocations}

The solution for this type of model is obtained backward, getting the solution for the long-run allocation first. Then this is used to compute expectations in the transitional state and obtain the medium-run solution. Finally, I use both medium- and long-run allocations to compute expectations in the crisis state and get the short-run solutions. Given the linearity of the model, to get the most general result, I derive these solutions assuming that both fiscal policy instruments $\left\{\hat{G}_{t}, \hat{\tau}_{t}^{I}\right\}$ are active in every state. According to each hypothesis made in the analysis that follows, one or the other instrument is muted.

The short- and medium-run allocations depend on the monetary policy adopted in the transitional state. Proposition 2.1 presents the results for those cases where the monetary authority keeps the nominal interest rate at a fixed level $i_{M}, \forall t \in\left[T_{\text {exit }}, T_{M}\right)$ different from that prescribed by the policy rule (2-3). ${ }^{12}$ Proposition 2.1 outlines the results if the monetary authority returns to rule (2-3) as soon as the crisis is over.

\footnotetext{
${ }^{12}$ Initially, I assume that $i_{M}=0$, so the monetary authority is expected to keep the nominal rate at zero even after the crisis is over. But in the next Chapter, where I examine the welfare implications of these policies, I also discuss what an optimal level for the nominal rate would be in the transitional state. That is why $i_{M}$ is kept in the derivations instead of already substituting it by zero.
} 
Proposition 2.1 Assume that the nominal interest rate zero lower bound is binding in the short run $\left(t \in\left[T_{0}, T_{\text {exit }}\right)\right)$ and that the following conditions hold:

$$
\begin{array}{ll}
(C 1) & \Gamma_{\mu \sigma} \equiv(1-\mu)(1-\beta \mu)-\mu \kappa \sigma>0 \\
(C 2) & \Gamma_{b \sigma} \equiv(1-b)(1-\beta b)-b \kappa \sigma>0 \\
(C 3) & \Gamma_{\phi} \equiv \phi_{\pi}+\frac{(1-\beta)}{\kappa} \phi_{y}>1 \\
(C 4) & r_{S}^{e}<-\Theta_{i_{M}}\left(\bar{r}-i_{M}\right)-\Theta_{G_{S}} G_{S}-\Theta_{\tau_{S}^{I}} \tau_{S}^{I}-\Theta_{G_{M}} G_{M}-\Theta_{\tau_{M}^{I}} \tau_{M}^{I} .
\end{array}
$$

If there is a transitional state generated by the monetary authority keeping the nominal interest rate at $i_{M}=0$, or at an optimally chosen level $i_{M}=i_{M}^{*}$, after the crisis is over $\left(\forall t \in\left[T_{\text {exit }}, T_{M}\right)\right)$, solutions for output, inflation and the nominal interest rate in each state can be obtained backward as follows:

(i) In the long run $\left(\forall t>T_{M}\right)$, with $r_{t}^{e}=\bar{r}$, there is a locally unique bounded solution such that $i_{L}=\bar{r}$, with $Y_{L}=\pi_{L}=0$.

(ii) There is a locally unique bounded medium-run solution $\left(\forall t \in\left[T_{\text {exit }}, T_{M}\right)\right)$, with $r_{t}^{e}=\bar{r}$ and $i_{t}=i_{M}$, such that

$$
\begin{aligned}
& Y_{M}=\Omega_{Y_{M}, i_{M}}\left(\bar{r}-i_{M}\right)+\Omega_{Y_{M}, G_{M}} G_{M}+\Omega_{Y_{M}, \tau_{M}^{I}} \tau_{M}^{I} \\
& \pi_{M}=\Omega_{\pi_{M}, i_{M}}\left(\bar{r}-i_{M}\right)+\Omega_{\pi_{M}, G_{M}} G_{M}+\Omega_{\pi_{M}, \tau_{M}^{I}} \tau_{M}^{I} .
\end{aligned}
$$

(iii) In the short run $\left(\forall t \in\left[T_{0}, T_{\text {exit }}\right)\right)$, with $r_{t}^{e}=r_{S}^{e}$, there is a locally unique bounded solution, such that $i_{t}=0$ and

$$
\begin{aligned}
& Y_{S}=\Omega_{Y_{S}, r_{S}^{e}} r_{S}^{e}+\Omega_{Y_{S}, G_{S}} G_{S}+\Omega_{Y_{S}, \tau_{S}^{I}} \tau_{S}^{I}+ \\
& \Omega_{Y_{S}, i_{M}}\left(\bar{r}-i_{M}\right)+\Omega_{Y_{S}, G_{M}} G_{M}+\Omega_{Y_{S}, \tau_{M}^{I}} \tau_{M}^{I} \\
& \pi_{S}=\Omega_{\pi_{S}, r_{S}^{e}} r_{S}^{e}+\Omega_{\pi_{S}, G_{S}} G_{S}+\Omega_{\pi_{S}, \tau_{S}^{I}} \tau_{S}^{I}+ \\
& \Omega_{\pi_{S}, i_{M}}\left(\bar{r}-i_{M}\right)+\Omega_{\pi_{S}, G_{M}} G_{M}+\Omega_{\pi_{S}, \tau_{M}^{I}} \tau_{M}^{I}
\end{aligned}
$$

where the analytical expressions for the coefficients $\Omega_{i, j}, i \in\left\{Y_{S}, \pi_{S}, Y_{M}, \pi_{M}\right\}$ and $j \in\left\{r_{s}^{e}, i_{M}, G_{S}, \tau_{S}^{I}, G_{M}, \tau_{M}^{I}\right\}$ and $\Theta_{k}, k \in\left\{i_{M}, G_{S}, \tau_{S}^{I}, G_{M}, \tau_{M}^{I}\right\}$, are defined in Appendix $B$ and depend on the structural parameters.

Proof. See Appendix B.1.

The analytical expression for the solution coefficients $\left(\Omega^{\prime} s\right)$ in equations (2-4) - (2-7) are presented in Tables 2.2 and 2.3. The proof of Proposition 2.1 is long and its details are presented in Appendix B.1. Nevertheless, there are a few aspects to be highlighted. The proof is divided into three parts. The first part discusses determinacy of the solution in each state and obtains conditions 
$(C 1)-(C 3)$, which are shown to be necessary for a determinate solution. Given the parameters $\beta, \sigma$ and $\kappa$, Condition $(C 1)$ requires the term $\Gamma_{\mu \sigma}$ in the denominator of short-run output and inflation to be positive, imposing an upper bound on the duration of the crisis state $(\mu)$. Likewise, Condition $(C 2)$ requires the term $\Gamma_{b \sigma}$ in the denominator of medium-run output and inflation coefficients to be also positive, imposing an upper bound on the length of the transitional state $(b)$. Condition $(C 3)$ is the regular determinacy condition for a New Keynesian model with monetary policy given by a rule like (2-3). It requires the term $\Gamma_{\phi}$ in the medium-run output and inflation coefficients to be positive and it is always satisfied given that I assume $\phi_{\pi}>1$ and $\phi_{y}>0$. The second part of the proof derives the analytical expressions for the solutions in each state (equations (2-4) - (2-7)), which are summarized in Tables 2.2 and 2.3 .

Table 2.2: Analytical expressions for medium-run solution coefficients from Proposition 2.1

\begin{tabular}{ccc}
\hline \hline & $Y_{M}$ & $\pi_{M}$ \\
\hline$\Omega_{*, i_{M}}$ & $\frac{(1-\beta b) \sigma}{\Gamma_{b \sigma}}$ & $\frac{\kappa \sigma}{\Gamma_{b \sigma}}$ \\
\hline$\Omega_{*, G_{M}}$ & $\frac{(1-b)(1-\beta b)-b \kappa \psi}{\Gamma_{b \sigma}}$ & $\frac{\kappa(1-b)\left(1-\psi \sigma^{-1}\right)}{\Gamma_{b \sigma}}$ \\
\hline$\Omega_{*, \tau_{M}^{I}}$ & $\frac{b \kappa \sigma \psi \chi^{I}}{\Gamma_{b \sigma}}$ & $\frac{(1-b) \kappa \psi \chi^{I}}{\Gamma_{b \sigma}}$ \\
\hline$\Gamma_{b \sigma} \equiv(1-b)(1-\beta b)-b \kappa \sigma$ & \\
\hline
\end{tabular}

Finally, the third part of the proof of Proposition 2.1 derives the restriction on the size of the shock $r_{S}^{e}$ and the stimulus policies that make the zero lower bound binding in the short run (Condition $(C 4)$ ). The coefficients in Condition $(C 4)$ are presented in Table 2.4 below. Note that if no fiscal instrument is used, $\hat{G}_{t}=\hat{\tau}_{t}^{I}=0, \forall t$, and monetary policy goes back to rule (2-3) right after the crisis is over (implying $i_{M}=\bar{r}$ ), this condition states that $r_{S}^{e}<0$. The condition derived here nests the one derived by Eggertsson (31). ${ }^{13}$ It is interesting to point out that this condition depends not only on monetary and fiscal policies adopted during the crisis but on those expected to be adopted in future states as well.

The values associated with each coefficient will be discussed in the next Section. But it is important to underline that the solution derived in this

${ }^{13}$ Assuming $b=0$ makes $\Theta_{i_{M}}=\Theta_{G_{M}}=\Theta_{\tau_{M}^{I}}=0$, shutting down the medium run. 
Table 2.3: Analytical expressions for short-run solution coefficients from Proposition 2.1

\begin{tabular}{|c|c|c|}
\hline & $Y_{S}$ & $\pi_{S}$ \\
\hline$\Omega_{*, r_{S}^{e}}$ & $\frac{(1-\beta \mu) \sigma}{\Gamma_{\mu \sigma}}$ & $\frac{\kappa \sigma}{\Gamma_{\mu \sigma}}$ \\
\hline$\Omega_{*, i_{M}}$ & $\frac{b(1-\mu)}{\Gamma_{\mu \sigma}}\left[\begin{array}{l}(1-\beta \mu) \Omega_{Y_{M}, i_{M}}+ \\
\sigma \Omega_{\pi_{M}, i_{M}}\end{array}\right]$ & $\frac{b(1-\mu)}{\Gamma_{\mu \sigma}}\left[\begin{array}{l}\kappa \Omega_{Y_{M}, i_{M}}+ \\
{[\beta(1-\mu)+\kappa \sigma] \Omega_{\pi_{M}, i_{M}}}\end{array}\right.$ \\
\hline$\Omega_{*, G_{S}}$ & $\frac{(1-\beta \mu)(1-\mu)-\mu \kappa \psi}{\Gamma_{\mu \sigma}}$ & $\frac{(1-\mu)\left(1-\psi \sigma^{-1}\right) \kappa}{\Gamma_{\mu \sigma}}$ \\
\hline$\Omega_{*, G_{M}}$ & $\frac{b(1-\mu)}{\Gamma_{\mu \sigma}}\left[\begin{array}{l}(1-\beta \mu)\left(\Omega_{Y_{M}, G_{M}}-1\right)+ \\
\sigma \Omega_{\pi_{M}, G_{M}}\end{array}\right]$ & $\frac{b(1-\mu)}{\Gamma_{\mu \sigma}}\left[\begin{array}{l}\kappa\left(\Omega_{Y_{M}, G_{M}}-1\right)+ \\
{[\beta(1-\mu)+\kappa \sigma] \Omega_{\pi_{M}, G_{M}}}\end{array}\right.$ \\
\hline$\Omega_{*, \tau_{S}^{I}}$ & $\frac{\mu \kappa \sigma \psi \chi^{I}}{\Gamma_{\mu \sigma}}$ & $\frac{(1-\mu) \kappa \psi \chi^{I}}{\Gamma_{\mu \sigma}}$ \\
\hline$\Omega_{*, \tau_{M}^{I}}$ & $\frac{b(1-\mu)}{\Gamma_{\mu \sigma}}\left[\begin{array}{l}(1-\beta \mu) \Omega_{Y_{M}, \tau_{M}^{I}}+ \\
\sigma \Omega_{\pi_{M}, \tau_{M}^{I}}\end{array}\right]$ & $\frac{b(1-\mu)}{\Gamma_{\mu \sigma}}\left[\begin{array}{l}\kappa \Omega_{Y_{M}, \tau_{M}^{I}} \tau_{M}^{I}+ \\
{[\beta(1-\mu)+\kappa \sigma] \Omega_{\pi_{M}, \tau_{M}^{I}}}\end{array}\right.$ \\
\hline$\Gamma_{\mu \sigma} \equiv$ & $-\mu)(1-\beta \mu)-\mu \kappa \sigma$ & \\
\hline
\end{tabular}

Table 2.4: Analytical expressions for the coefficients in Condition (C4) from Proposition 2.1

\begin{aligned} & \hline$\Theta_{i_{M}}= \frac{(1-\mu) b}{\Gamma_{\mu \phi}}\left\{\begin{array}{l}{\left[\phi_{\pi} \kappa+(1-\beta \mu) \phi_{y}\right] \Omega_{Y_{M}, i_{M}}+} \\ {\left[\phi_{y} \sigma+(\beta(1-\mu)+\kappa \sigma) \phi_{\pi}\right] \Omega_{\pi_{M}, i_{M}}}\end{array}\right\} \\ &$\hline$\Theta_{G_{S}}=\frac{(1-\mu)\left(1-\psi \sigma^{-1}\right) \phi_{\pi} \kappa+[(1-\mu)(1-\beta \mu)-\mu \kappa \psi] \phi_{y}}{\Gamma_{\mu \phi}} \\ &$\hline$\Theta_{\tau_{S}^{I}}=\frac{(1-\mu) \phi_{\pi}+\mu \phi_{y} \sigma}{\Gamma_{\mu \phi}} \kappa \psi \chi^{I} \\ &$\hline$\Theta_{G_{M}}=\frac{(1-\mu) b}{\Gamma_{\mu \phi}}\left\{\begin{array}{l}{\left[\phi_{\pi} \kappa+(1-\beta \mu) \phi_{y}\right]\left(\Omega_{Y_{M}, G_{M}}-1\right)+} \\ {\left[\phi_{y} \sigma+(\beta(1-\mu)+\kappa \sigma) \phi_{\pi}\right] \Omega_{\pi_{M}, G_{M}}}\end{array}\right\} \\ &$\hline$\Theta_{\tau_{M}^{I}}=\frac{(1-\mu) b}{\Gamma_{\mu \phi}\left\{\begin{array}{c}{\left[\phi_{\pi} \kappa+(1-\beta \mu) \phi_{y}\right] \Omega_{Y_{M}, \tau_{M}^{I}}+} \\ {\left[\phi_{y} \sigma+(\beta(1-\mu)+\kappa \sigma) \phi_{\pi}\right] \Omega_{\pi_{M}, \tau_{M}^{I}}^{I}}\end{array}\right\}} \\ &$\hline$\Gamma_{\mu \phi} \equiv(1-\beta \mu)\left(1-\mu+\sigma \phi_{y}\right)+\left(\phi_{\pi}-\mu\right) \kappa \sigma\end{aligned}$

proposition nests the benchmark case, presented by Eggertsson (31). Given the linearity of the model, it is easy to see that, if we assume there is no transitional state $(b=0)$, the economy jumps straight to the steady state, 
right after the crisis is over. ${ }^{14}$ Hence, the short-run solution obtained from equations (2-6) and (2-7) are reduced to

$$
\begin{aligned}
& Y_{S}=\Omega_{Y_{S}, r_{S}^{e}} r_{S}^{e}+\Omega_{Y_{S}, G_{S}} G_{S}+\Omega_{Y_{S}, \tau_{S}^{I}} \tau_{S}^{I}, \\
& \pi_{S}=\Omega_{\pi_{S}, r_{S}^{e}} r_{S}^{e}+\Omega_{\pi_{S}, G_{S}} G_{S}+\Omega_{\pi_{S}, \tau_{S}^{I}} \tau_{S}^{I},
\end{aligned}
$$

where the multipliers $\Omega_{i, j}, i \in\left\{Y_{S}, \pi_{S}\right\}$ and $j \in\left\{r_{s}^{e}, G_{S}, \tau_{S}^{I}\right\}$ are precisely those derived by Eggertsson (31). Proposition 2.2 presents the solution allocations for the crisis and transitional states if the monetary authority goes back to rule (2-3) as soon as the crisis is over, but fiscal stimulus continues in the transitional state.

Proposition 2.2 Assume that the nominal interest rate zero lower bound is binding in the short run $\left(t \in\left[T_{0}, T_{\text {exit }}\right)\right)$ and that the following conditions hold:

$$
\begin{array}{ll}
(C 1) & \Gamma_{\mu \sigma} \equiv(1-\mu)(1-\beta \mu)-\mu \kappa \sigma>0, \\
(C 2) & \Gamma_{b \sigma} \equiv(1-b)(1-\beta b)-b \kappa \sigma>0, \\
(C 3) & \Gamma_{\phi} \equiv \phi_{\pi}+\frac{(1-\beta)}{\kappa} \phi_{y}>1, \\
\left(C 4^{\prime}\right) & r_{S}^{e}<-\Theta_{G_{S}} G_{S}-\Theta_{\tau_{S}^{I}} \tau_{S}^{I}-\Theta_{G_{M}}^{T} G_{M}-\Theta_{\tau_{M}^{I}}^{T} \tau_{M}^{I} .
\end{array}
$$

If there is a transitional state generated by the fiscal authority keeping the stimulus provided in the short run for a few periods after the crisis is over $\left(\forall t \in\left[T_{\text {exit }}, T_{M}\right)\right)$, while monetary policy returns to rule (2-3) as soon as $r_{t}^{e}$ returns to $\bar{r}$, the solutions in each state can be obtained backward as follows:

(i) In the long run, there is a locally unique bounded solution $\left(\forall t>T_{M}\right)$, with $r_{t}^{e}=\bar{r}$, such that $i_{L}=\bar{r}$ and $Y_{L}=\pi_{L}=0$.

(ii) There is a locally unique bounded medium-run solution $\left(\forall t \in\left[T_{\text {exit }}, T_{M}\right)\right)$, with $r_{t}^{e}=\bar{r}$, such that

$$
\begin{aligned}
Y_{M}^{T} & =\Omega_{Y_{M}, G_{M}}^{T} G_{M}+\Omega_{Y_{M}, \tau_{M}^{I}}^{T} \tau_{M}^{I}, \\
\pi_{M}^{T} & =\Omega_{\pi_{M}, G_{M}}^{T} G_{M}+\Omega_{\pi_{M}, \tau_{M}^{I}}^{T} \tau_{M}^{I}, \\
i_{M}^{T} & =\bar{r}+\phi_{\pi} \pi_{M}^{T}+\phi_{y} Y_{M}^{T} .
\end{aligned}
$$

\footnotetext{
${ }^{14}$ It is important to note that making $G_{M}=\tau_{M}^{I}=0$ is not enough to rule out the transitional state. The isolated action of the monetary authority keeping the nominal interest rate at an $i_{M}$ different from that prescribed by the rule (2-3) for a few periods after the crisis is over can still generate it. Eliminating fiscal instruments in the medium run would still leave the effect of the term $\left(\bar{r}-i_{M}\right)$. As can be seen in Table 2.3, for $i \in\left\{Y_{S}, \pi_{S}\right\}$, making $b=0$ implies that $\Omega_{i, G_{M}}=\Omega_{i, \tau_{M}^{I}}=\Omega_{i, i_{M}}=0$.
} 
(iii) In the short run $\left(\forall t \in\left[T_{0}, T_{\text {exit }}\right)\right)$, with $r_{t}^{e}=r_{S}^{e}$, there is a locally unique bounded solution, such that $i_{s}=0$ and

$$
\begin{aligned}
& Y_{S}^{T}=\Omega_{Y_{S}, r_{S}^{e}} r_{S}^{e}+\Omega_{Y_{S}, G_{S}} G_{S}+\Omega_{Y_{S}, \tau_{S}^{I}} \tau_{S}^{I}+ \\
& \Omega_{Y_{S}, G_{M}}^{T} G_{M}+\Omega_{Y_{S}, \tau_{M}^{I}}^{T} \tau_{M}^{I} \\
& \pi_{S}^{T}=\Omega_{\pi_{S}, r_{S}^{e}} r_{S}^{e}+\Omega_{\pi_{S}, G_{S}} G_{S}+\Omega_{\pi_{S}, \tau_{S}^{I}} \tau_{S}^{I}+ \\
& \Omega_{\pi_{S}, G_{M}}^{T} G_{M}+\Omega_{\pi_{S}, \tau_{M}^{I}}^{T} \tau_{M}^{I}
\end{aligned}
$$

where the analytical expressions for the coefficients $\Omega_{i, j}, i \in\left\{Y_{S}, \pi_{S}\right\}$ and $j \in\left\{r_{S}^{e}, G_{S}, \tau_{S}^{I}\right\}$, and $\Theta_{k}, k \in\left\{G_{S}, \tau_{S}^{I}\right\}$, are the same as those defined in Proposition 1. The expressions for the coefficients $\Omega_{m, n}^{T}, m \in\left\{Y_{S}, \pi_{S}, Y_{M}, \pi_{M}\right\}$ and $n \in\left\{G_{M}, \tau_{M}^{I}\right\}$, and $\Theta_{h}^{T}, k \in\left\{G_{M}, \tau_{M}^{I}\right\}$ are defined in the appendix and depend on the structural parameters.

Proof. See Appendix B.2.

Following the same rationale for the proof of Proposition 2.1, the proof of Proposition 2.2 is also divided into three parts. The first part shows that Conditions $(C 1)-(C 3)$ are also necessary for determinacy of the solutions. The second part derives the analytical expressions for the solutions in each state (equations (2-8) -(2-12)). Table 2.5 presents the coefficients for mediumrun output and inflation in this case (equations (2-8) - (2-9), while Table 2.6 shows only the coefficients in the second line of equations (2-11) - (2-12), since

\begin{tabular}{|c|c|c|}
\hline & $Y_{M}$ & $\pi_{M}$ \\
\hline$\Omega_{*, G_{M}}^{T}$ & $\frac{(1-b)(1-\beta b)+\left(\phi_{\pi}-b\right) \kappa \psi}{\Gamma_{\phi}}$ & $\frac{\kappa\left[(1-b)\left(1-\psi \sigma^{-1}\right)-\psi \phi_{y}\right]}{\Gamma_{\phi}}$ \\
\hline$\Omega_{*, \tau_{M}^{I}}^{T}$ & $-\frac{\left(\phi_{\pi}-b\right) \kappa \sigma \psi \chi^{I}}{\Gamma_{\phi}}$ & $\frac{\left(1-b+\sigma \phi_{y}\right) \kappa \psi \chi^{I}}{\Gamma_{\phi}}$ \\
\hline$\Gamma_{\phi} \equiv \phi$ & $+\frac{(1-\beta)}{\kappa} \phi_{y}$ & \\
\hline
\end{tabular}
the coefficients in the first line are the same as those presented in Table 2.3.

Table 2.5: Analytical expressions for medium-run solution coefficients from Proposition 2.2

Finally, the third part of the proof of Proposition 2.2 derives the condition that guarantees that the zero lower bound is binding in the short run $\left(C 4^{\prime}\right)$. Table 2.7 shows the analytical expressions for the coefficients $\Theta_{G_{M}}^{T}$ and $\Theta_{\tau_{M}^{I}}^{T}$ in Condition $\left(C 4^{\prime}\right)$. The expressions for $\Theta_{G_{S}}$ and $\Theta_{\tau_{S}^{I}}$ are the same as those for Proposition 2.1, which are presented in Table 2.4. 
Table 2.6: Analytical expressions for short-run solution coefficients from Proposition 2.2

\begin{tabular}{ccc}
\hline \hline & $Y_{S}$ & $\pi_{S}$ \\
\hline$\Omega_{*, G_{M}}^{T}$ & $\frac{b(1-\mu)}{\Gamma_{\mu \sigma}}\left[\begin{array}{l}\left.(1-\beta \mu)\left(\Omega_{Y_{M}, G_{M}}^{T}-1\right)+\right] \\
\sigma \Omega_{\pi_{M}, G_{M}}^{T}\end{array}\right.$ & $\frac{b(1-\mu)}{\Gamma_{\mu \sigma}}\left[\begin{array}{l}\kappa\left(\Omega_{Y_{M}, G_{M}}^{T}-1\right)+ \\
{[\beta(1-\mu)+\kappa \sigma] \Omega_{\pi_{M}, G_{M}}^{T}}\end{array}\right]$ \\
\hline$\Omega_{*, \tau_{M}^{I}}^{T}$ & $\frac{b(1-\mu)}{\Gamma_{\mu \sigma}}\left[\begin{array}{l}(1-\beta \mu) \Omega_{Y_{M}, \tau_{M}^{I}}^{T}+ \\
\sigma \Omega_{\pi_{M}, \tau_{M}^{I}}^{T}\end{array}\right]$ & $\frac{b(1-\mu)}{\Gamma_{\mu \sigma}}\left[\begin{array}{l}\kappa \Omega_{Y_{M}, \tau_{M}^{I}}^{T} \tau_{M}^{I}+ \\
{[\beta(1-\mu)+\kappa \sigma] \Omega_{\pi_{M}, \tau_{M}^{I}}^{T}}\end{array}\right]$ \\
\hline$\Gamma_{\mu \sigma} \equiv(1-\mu)(1-\beta \mu)-\mu \kappa \sigma$ & \\
\hline
\end{tabular}

Table 2.7: Analytical expressions for the coefficients in Condition (C4') from Proposition 2.2

$$
\begin{gathered}
\Theta_{G_{M}}^{T}=\frac{(1-\mu) b}{\Gamma_{\mu \phi}}\left\{\begin{array}{l}
{\left[\phi_{\pi} \kappa+(1-\beta \mu) \phi_{y}\right]\left(\Omega_{Y_{M}, G_{M}}^{T}-1\right)+} \\
{\left[\phi_{y} \sigma+(\beta(1-\mu)+\kappa \sigma) \phi_{\pi}\right] \Omega_{\pi_{M}, G_{M}}^{T}}
\end{array}\right\} \\
\Theta_{\tau_{M}^{I}}^{T}=\frac{(1-\mu) b}{\Gamma_{\mu \phi}}\left\{\begin{array}{l}
{\left[\phi_{\pi} \kappa+(1-\beta \mu) \phi_{y}\right] \Omega_{Y_{M}, \tau_{M}^{I}}^{T}+} \\
{\left[\phi_{y} \sigma+(\beta(1-\mu)+\kappa \sigma) \phi_{\pi}\right] \Omega_{\pi_{M}, \tau_{M}^{I}}^{T}}
\end{array}\right\} \\
\Gamma_{\mu \phi} \equiv(1-\beta \mu)\left(1-\mu+\sigma \phi_{y}\right)+\left(\phi_{\pi}-\mu\right) \kappa \sigma
\end{gathered}
$$

Proposition 2.2 allows analyzing a temporary continuation of the fiscal stimulus with the response of monetary policy through rule (2-3). Woodford (33) analyses this case, but he only deals with government spending and does not discuss the association with an accommodative monetary policy in the transitional state.

In what follows, given the results from Propositions 2.1 and 2.2, we analyse the properties of the coefficients $\left(\Omega^{\prime} s\right)$ and the impact of different combinations of policies adopted during the crisis, and expected to be adopted in the future, on output and inflation in each state under the chosen parametrization for the model.

\section{5}

\section{Analysis of Fiscal Policy Multipliers}

To discuss the behaviour of multipliers in this Section, and the solution allocations for output and inflation in each state in the next, I set the model's parameters ${ }^{15}$ according to the values used by Eggertsson (31).

${ }^{15}$ For a quarterly model I assume: $\sigma^{-1}=1.1599 ; \beta=0.9970 ; \omega=1.5692 ; \alpha=0.7747$; $\theta=12.7721 ; \phi_{\pi}=1.5 ; \phi_{y}=0.125 ; \bar{\tau}^{I}=0.2 ; r_{S}^{e}=-0.0104 ; \mu=0.903$. Probability $\mu$ is set to the maximum value that satisfies Condition $(C 1)$, which corresponds to an average crisis duration of 10 quarters. The analogous Condition $(C 2)$ establishes the same maximum value for the probability $b$, thus throughout the analysis we allow it to vary in the interval $[0,0.9]$. 
Given the results in the previous Section, I first look at the isolated impact of each policy instrument on output and inflation in each state. The relations derived in Propositions 2.1 and 2.2 are linear functions of government spending and income taxes in each state $\left(G_{S}, \tau_{S}^{I}, G_{M}\right.$ and $\left.\tau_{M}^{I}\right)$, the shock $\left(r_{s}^{e}\right)$ and the gap between steady-state interest rate and medium-run interest rate $\left(\bar{r}-i_{M}\right)$. The coefficients that appear in equations $(2-8)-(2-12)$ represent output and inflation multipliers $\left(\Omega^{\prime} s\right)$, where some of them depend on the monetary policy adopted in the transitional state.

Table 2.8 presents these coefficients' values under the assumed parameters, as a function of probability $b$. First I look at the direct impact of the shock $\left(r_{s}^{e}\right)$ on crisis-state output and inflation $\left(\Omega_{Y_{S}, r_{S}^{e}}=28.8\right.$ and $\Omega_{\pi_{S}, r_{S}^{e}}=2.5$, respectively). These coefficients do not depend on the probability associated with the transitional state $(b)$, but they are increasing in the duration of the shock (determined by probability $\mu$ ) as illustrated in Figure 2.3.

Table 2.8: Solution coefficients in each state as a function of $b$

\begin{tabular}{rrrrr}
\hline \hline & \multicolumn{1}{c}{$Y_{S}$} & \multicolumn{1}{c}{$\pi_{S}$} & $Y_{M}$ & \multicolumn{1}{c}{$\pi_{M}$} \\
\hline \hline$\Omega_{*, r_{S}^{e}}$ & 28.8 & 2.5 & & \\
$\Omega_{*, G_{S}}$ & 2.3 & 0.2 & & \\
$\Omega_{*, \tau_{S}^{I}}$ & 1.0 & 0.1 & & \\
$\Omega_{*, i_{M}}$ & {$[0.0,123.2]$} & {$[0.0,12.4]$} & {$[0.9,24.5]$} & {$[0.0,2.1]$} \\
$\Omega_{*, G_{M}}$ & {$[0.0,6.5]$} & {$[0.0,0.7]$} & {$[1.0,2.1]$} & {$[0.0,0.1]$} \\
$\Omega_{*, G_{M}}^{T}$ & {$[0.0,-1.4]^{\dagger}$} & {$[0.0,-0.1]^{\dagger}$} & {$[0.9,0.5]$} & {$[0.004,0.003]$} \\
$\Omega_{*, \tau_{M}^{I}}$ & {$[0.0,6.2]$} & {$[0.0,0.7]$} & {$[0.0,0.9]$} & {$[0.0,0.1]$} \\
$\Omega_{*, \tau_{M}^{I}}^{T}$ & {$[0.0,0.6]$} & {$[0.0,0.1]$} & {$[0.0,-0.1]^{\dagger}$} & {$[0.0,0.03]$} \\
\hline
\end{tabular}

Note: The intervals marked with $\dagger$ are decreasing in $b$. The others are increasing in $b$. The ${ }^{*}$ symbol represents the variables in each column.

As discussed in Section 2.4, if agents expect a continuation of the fiscal stimulus and/or an accommodative monetary policy after the crisis is over, this generates a transitional state that also affects the short-run allocations. If there is a positive probability associated with the monetary authority deciding to keep nominal interest rate at zero after $r_{s}^{e}$ returns to $\bar{r}$, the term $\left(\bar{r}-i_{M}\right)$ impacts not only medium-run output and inflation $\left(\Omega_{Y_{M}, i_{M}}=[0.9,24.5]\right.$ and $\Omega_{\pi_{M}, i_{M}}=[0.0,2.1]$, respectively $)$, but also their short-run levels $\left(\Omega_{Y_{S}, i_{M}}=[0.0,123.2]\right.$ and $\Omega_{\pi_{S}, i_{M}}=[0.0,12.4]$, respectively). Its effects on these allocations are positive and increasing in the expected duration 
Figure 2.3: Short-run coefficients as a function of probability $\mu$
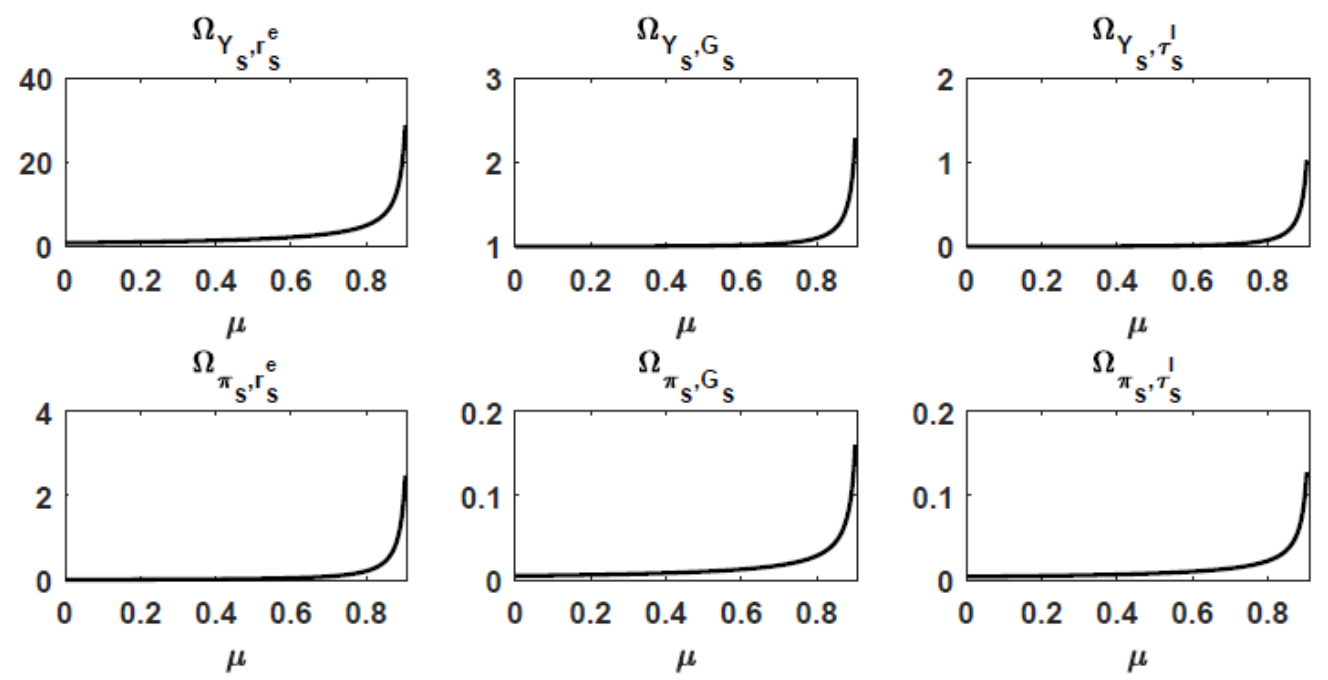

Note: This figure presents the responses of short-run output and inflation to the shock $\left(r_{S}{ }^{e}\right)$ and to fiscal policies implemented during the crisis through increases in government spending $(G)$ or income taxes $\left(\tau^{I}\right)$ as a function of its duration detemined by probability $(\mu)$

of the transitional state, determined by probability $b$, as seen in Figure 2.4. This means that monetary stimulus provided after the crisis is over can play a significant role in attenuating the depth of the recession caused by the shock. This happens because it increases output and generates inflation in the medium run, producing a positive impact on short-run output and inflation through expectations.

A point worth mentioning is the size of these coefficients when $\mu$ and $b$ get very large. A long lasting crisis (higher $\mu$ ) has a large impact on shortrun output and inflation. Besides, an accommodative monetary policy in the transitional state can also have significant impacts on short- and medium-run output and inflation, if it is expected to last for a long period (higher $b$ ). Note that, the larger $\mu$ and $b$, smaller are $\Gamma_{\mu \sigma}$ and $\Gamma_{b \sigma}$, respectively, approximating the model to its determinacy frontier.

The coefficients on fiscal policy instruments reflect their isolated impact on each state's allocation. These are the multipliers which most of the literature focuses the discussion on. In what follows, the multipliers for each fiscal instrument are examined separately.

\subsection{1}

\section{Government Spending Multipliers}

The impact of fiscal stimuli provided during the crisis (which are independent of $b$, but highly dependent on $\mu$ ) replicates the multipliers found in Eggertsson (31). He shows that, when the zero lower bound is binding, an 
Figure 2.4: Shock and interest rate coefficients as a function of $b$
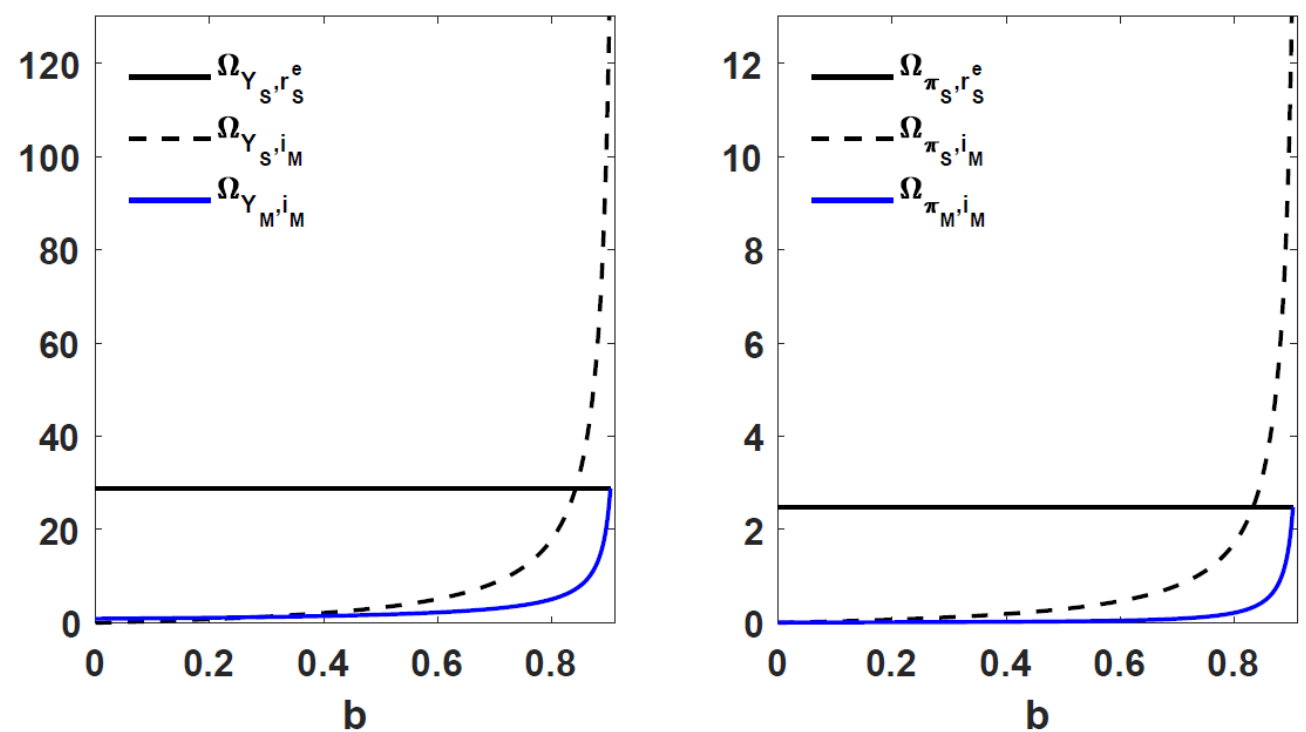

Note: This figure presents the behavior of the coefficients in response to the shock $\left(r_{S}^{e}\right)$ and to the difference between long- and medium-run interest rates $\left(\bar{r}-i_{M}\right)$ as a function of the probability associated with the transitional state $(b)$.

increase in government spending has a significant impact on crisis-state output $\left(\Omega_{Y_{S}, G_{S}}=2.3\right)$ and inflation $\left(\Omega_{\pi_{S}, G_{S}}=0.2\right)$, attenuating the depth of the recession. In fact, providing the same stimulus in normal times ${ }^{16}$, for exactly the duration of the crisis (determined by the probability $\mu$ ), would produce a smaller impact on output and inflation $\left(\Omega_{Y_{S}, G_{S}}^{N}=0.5\right.$ and $\Omega_{\pi_{S}, G_{S}}^{N}=0.003$, respectively). ${ }^{17}$

As Woordford (33) points out, the short-run government spending multiplier is the highest when it is needed the most, when fiscal stimulus becomes more urgent since interest rate cuts can no longer stimulate aggregate demand. He also observes that, if government spending is increased for just one period during the crisis, ${ }^{18,19}$ the output multiplier would be $\Omega_{Y_{S}, G_{S}}^{1}=1$. This means that from the 2.3 increase in output caused by the fiscal stimulus, 1 comes from the increase in government spending in the first quarter, while 1.3 results from the expected spending increase in the following quarters. The same happens to inflation, which would respond with 0.005 of the rise in the first quarter and a significant part, 0.155 , coming from the increase in government spending in future periods, when the zero lower bound is still binding.

\footnotetext{
${ }^{16}$ When the zero lower bound is not binding and policy rule (2-3) determines the nominal interest rate.

${ }^{17}$ The multipliers in normal times are obtained by using the coefficients from equations (2-8) and (2-9)and assuming $b=\mu$.

${ }^{18}$ To obtain the 1-quarter multiplier, consider $\mu=0$ in $\Omega_{Y_{S}, G_{S}}$ and $\Omega_{\pi_{S}, G_{S}}$.

${ }^{19}$ If government spending increases for one period only in normal times, the multipliers would be $\Omega_{Y_{S}, G_{S}}^{N_{1}}=0.90$ and $\Omega_{\pi_{S}, G_{S}}^{N_{1}}=0.004$.
} 
One important observation regarding the multipliers obtained by Eggertsson (31) is that they are highly dependent on the duration of the crisis $(\mu)$. Figure 2.3 shows the responses of crisis-state output and inflation $\left(Y_{S}, \pi_{S}\right)$ to the short-run fiscal instruments $\left(G_{S}, \tau_{S}^{I}\right)$ as a function of probability $\mu$. We see the significant increase in these coefficients towards the end of the interval. Therefore, one should keep in mind that we are working with the highest values of these coefficients once we assume $\mu=0.903$, which implies an average crisis duration of 10 quarters. It is worth highlighting that this is a limitation of the framework used here, since the maximum value allowed for the probability $\mu$, due to determinacy requirements as discussed in Propositions 2.1 and 2.2, does not admit a longer crisis spell. ${ }^{20}$

I now turn to the analysis of how expectations regarding future policies affect short-run output and inflation. The coefficients discussed are those presented in Table 2.8 and Figure 2.5. The impact of agents' expectations that the fiscal authority keeps the stimulus when the zero lower bound is no longer binding depends on how the monetary authority accommodates it. The association of higher government spending, with the nominal interest rate kept at $i_{M}=0$, after the crisis is over, generates an impact that is positive and increasing in $b$ on both transitional-state output $\left(\Omega_{Y_{M}, G_{M}} \in[1.0,2.1]\right)$ and inflation $\left(\Omega_{\pi_{M}, G_{M}} \in[0,0.1]\right)^{21}$. It also has positive effects on short-run output $\left(\Omega_{Y_{S}, G_{M}} \in[0,6.5]\right)$ and inflation $\left(\Omega_{\pi_{S}, G_{M}} \in[0,0.7]\right)$, which are significantly increasing in the probability $b$ as well. It is interesting to note that for high values of $b$, these multipliers get even more substantial than those observed for the short-run stimulus $\left(\Omega_{Y_{S}, G_{S}}\right.$ and $\left.\Omega_{\pi_{S}, G_{S}}\right) \cdot{ }^{22}$ On the other hand, if the monetary authority responds to the continuation of the government spending stimulus through policy rule 2-3, the impacts on medium-run output $\left(\Omega_{Y_{M}, G_{M}}^{T}\right.$ $\left.\in[0.9,0.5]^{\dagger}\right)$ and inflation $\left(\Omega_{\pi_{M}, G_{M}}^{T} \in[0.004,0.003]^{\dagger}\right)$ are still positive ${ }^{23}$, but decreasing in $b$ and smaller than those observed with an accommodative monetary policy. In this case, the short-run impact is negative and decreasing in $b\left(\Omega_{Y_{S}, G_{M}}^{T} \in[0,-1.4]^{\dagger}\right.$ and $\Omega_{\pi_{S}, G_{M}}^{T} \in[0,-0.1]^{\dagger}$ for output and inflation, respectively). This happens because output increases less than one-for-one with

${ }^{20}$ Probably, back in $2010-2011$, predicting an average expectation of 2.5 years of interest rates at the zero lower bound seemed very pessimistic. However, looking for how long the Fed Funds rate was maintained near zero (around 5 years), it might not have been so bad.

${ }^{21}$ Note that these multipliers accurately replicate the behavior of short-run multipliers varying $\mu$. The only difference is that we assume the maximum value for $b=0.90$, slightly smaller than $\mu=0.903$. Making $b=\mu$ gets the top of the interval equal to the short-run multipliers. We set $\mu=0.903$ to make the result comparable to those obtained by Eggertsson (31). It is just a matter of convenience that we do not extend the interval for $b$ until the value of $\mu$.

${ }^{22}$ This happens if $b \geq 0.87$ for output and $b \geq 0.85$ for inflation.

${ }^{23}$ The marker $\dagger$ highlights the intervals which are decreasing in $b$. 
Figure 2.5: Government Spending multipliers as a function of $b$
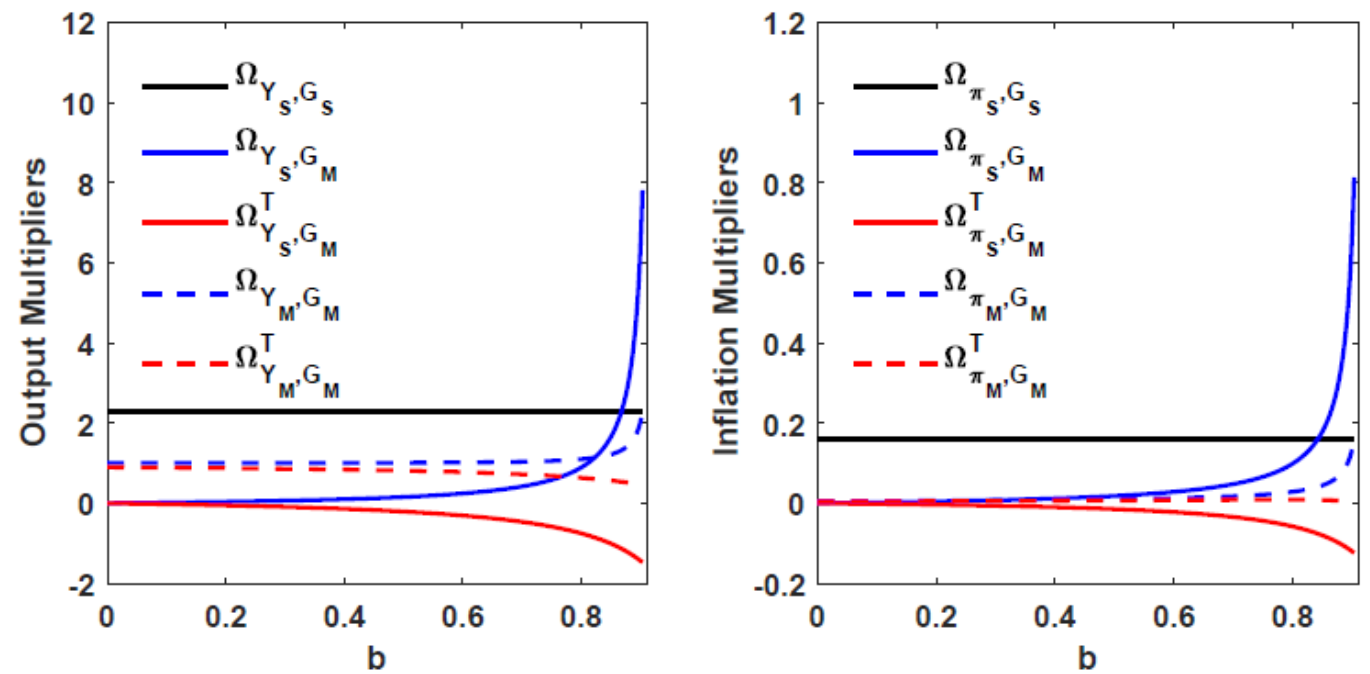

Note: This figure presents the responses of output and inflation to a fiscal stimulus implemented through an increase in government spending $(G)$, in each state, according to Propositions 1 and 2, as a function of the probability associated with the transtional state $(b)$.

the increase in government spending in the transitional state, accompanied by a small rise in inflation. Therefore, the stimulus generated in the medium run is small compared to the cost of higher government spending. This is in line with the result obtained by Woordford(33). His multiplier corresponds to $\left(\Omega_{Y_{S}, G_{S}}+\Omega_{Y_{S}, G_{M}}^{T}\right) \in[2.3,0.9]^{\dagger}$ for $b \in[0,0.9]$. He gets a negative total multiplier with a transitional-state probability higher than 0.91 , which is not allowed here. This also helps to understand the low values for the spending multipliers found by Cogan et.al. (40), since they assume a permanent increase in government spending and a large part of the stimulus takes place when monetary policy is no longer accommodating it.

\subsection{2}

\section{Income Tax Multipliers}

In general, one would expect that an increase in income taxes would reduce output and increase inflation. However, Eggertsson (31) shows the surprising result that, when the zero lower bound is binding, an increase in income taxes has a positive effect on short-run output ${ }^{24}\left(\Omega_{Y_{S}, \tau_{S}^{I}}=1.0\right)$, in contrast with the negative multiplier observed in normal times $\left(\Omega_{Y_{S}, \tau_{S}^{I}}^{N}=-0.1\right)$. The impact on inflation $\left(\Omega_{\pi_{S}, \tau_{S}^{I}}=0.1\right)$ is also more substantial than in normal

${ }^{24}$ This multiplier $\left(\Omega_{Y_{S}, \tau_{S}^{I}}=\frac{\mu \kappa \sigma \psi \chi^{I}}{(1-\mu)(1-\beta \mu)-\mu \kappa \sigma}\right)$ is always positive and increasing on the crisis' duration $(\mu)$ as seen in Figure 2.3. 
times $^{25}\left(\Omega_{\pi_{S}, \tau_{S}^{I}}^{N}=0.03\right)$. As seen in Figure 2.3, these multipliers are highly dependent on the value of probability $\mu$ which is set at the upper end of the feasible interval.

Eggertsson (31) explains that the output multiplier is now positive because, when the zero lower bound is binding, the short-run demand curve becomes upward slopping. ${ }^{26}$ This implies that an increase in taxes in the short run creates inflationary pressures, increasing inflation expectations in every state where the zero lower bound is still binding. This reduces the real interest rate, making current consumption cheaper and raising demand. It is important to point out that this result is particular to the model setup assumed. Eggertsson (31) observes that income taxes may have a direct effect on demand if one assumes that a fraction of workers and/or firms are liquidity constrained, for example.

I show that if income taxes are kept at a higher level after the crisis is over, associated with an accommodative monetary policy, this generates a transitional state where the economy is stimulated, with positive output and inflation multipliers $\left(\Omega_{Y_{M}, \tau_{M}^{I}} \in[0.0,0.9]\right.$ and $\Omega_{\pi_{M}, \tau_{M}^{I}} \in[0.0,0.1]$, respectively), which are increasing in $b$. This is illustrated in Figure 2.6. Again, these are equivalent to the short-run multipliers if we were varying $\mu$. This policy also has a positive impact on crisis-state output $\left(\Omega_{Y_{S}, \tau_{M}^{I}} \in[0.0,6.2]\right)$ and inflation $\left(\Omega_{\pi_{S}, \tau_{M}^{I}} \in[0.0,0.65]\right)$. These short-run multipliers are increasing in $b$, and they get larger than the effect of the short-run increase in taxes for higher levels of this probability ( $b \geq 0.84$ for output and $b \geq 0.85$ for inflation).

On the other hand, if the monetary authority goes back to following policy rule (2-3) as soon as the crisis is over, the continuation of higher income taxes decreases output $\left(\Omega_{Y_{M}, \tau_{M}^{I}}^{T} \in[0.0,-0.1]^{\dagger}\right)$ and causes inflation to increase less $\left(\Omega_{\pi_{M}, \tau_{M}^{I}}^{T} \in[0.0,0.03]\right)$ in the transitional state. The effects on short-run output and inflation are still positive $\left(\Omega_{Y_{S}, \tau_{M}^{I}} \in[0.0,0.6]\right.$ and $\Omega_{\pi_{S}, \tau_{M}^{I}} \in$ $[0.0,0.1]$, respectively), but smaller than those obtained when monetary policy accommodates the fiscal stimulus in the medium run.

It is important to note that these multipliers should be carefully interpreted since it is the interplay between policies that will determine the levels of output and inflation in each state. This is the topic of next Section.

\footnotetext{
${ }^{25}$ Again, the multipliers in normal times are computed by assuming the expected stimulus duration (given by $b$ ) to be the same of the crisis (determined by the probability $\mu$ ) in equations (2-8) and (2-9). If the stimulus was provided for just one period, its impact on output and inflation would be approximately zero during normal times or the crisis state.

${ }^{26}$ This can be seen in the derivation of the short-run solution in the proof of Proposition 2.1. Equation (B-13) shows that the slope of the aggregate demand curve is given by $\frac{\mu \sigma}{(1-\mu)}$.
} 
Figure 2.6: Taxes multipliers as a function of $b$
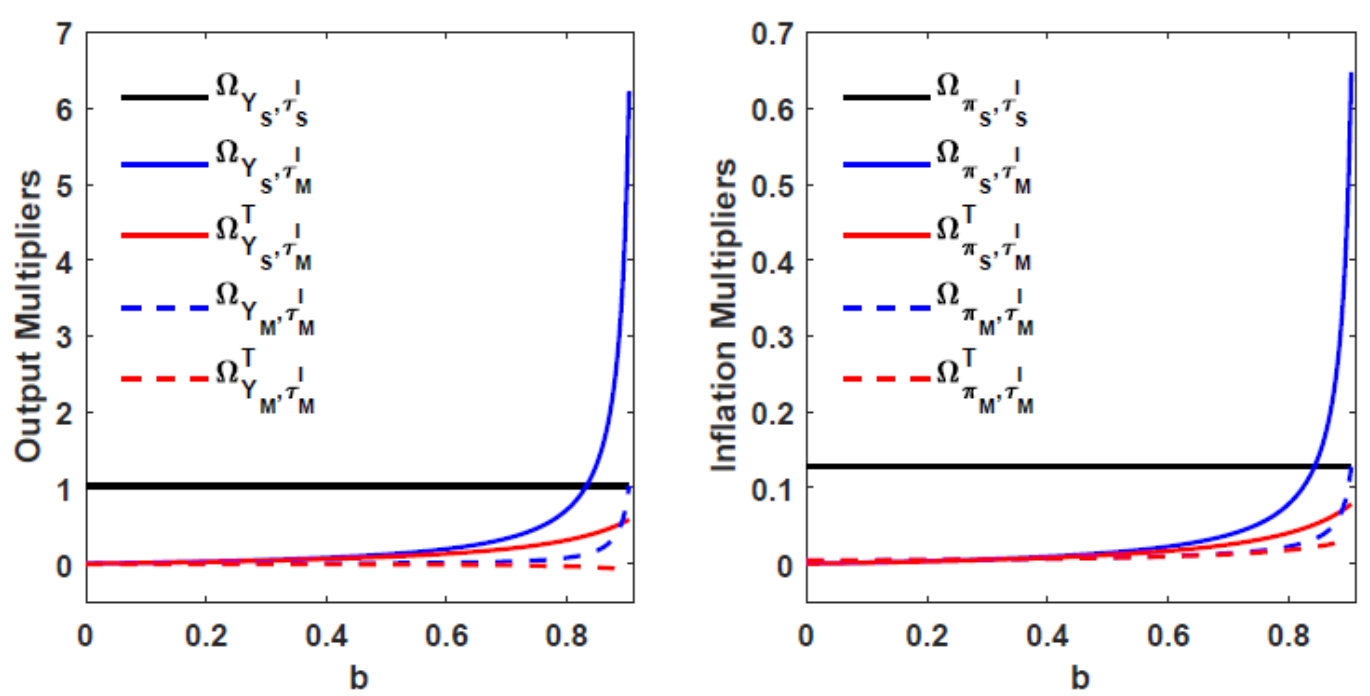

Note: This figure presents the responses of output and inflation to a fiscal stimulus implemented through an increase in income taxes $\left(\tau^{I}\right)$, in each state, according to Propositions 1 and 2 , as a function of the probability associated with the transitional state $(b)$.

\section{6}

\section{Analysis of Different Policies' Combinations}

I now discuss how the interaction of policies carried out during the crisis, their implementation timing and expectations regarding future policies affect the depth of the recession caused by a shock that makes the zero lower bound binding. I look at their effects on output and inflation in each state.

Before analysing the various cases, I point out to a result that, associated with those obtained in Propositions 2.1 and 2.2, is helpful to understand the mechanisms behind the effects obtained for output and inflation in each state. In the derivation of solution allocations in the proofs of these propositions, output and inflation are expressed as functions of the shock $\left(r_{S}^{e}\right)$, the gap between steady-state interest rate and medium-run interest rate $\left(\bar{r}-i_{M}\right)$ and the fiscal instruments in each state of the economy $\left(G_{S}, \tau_{S}^{I}, G_{M}\right.$ and $\left.\tau_{M}^{I}\right)$. This is convenient because it permits discussing the impact of each instrument on the levels of output and inflation. However, an intermediate step in the proof of Proposition 2.1 allows expressing short-run output and inflation as functions of output, inflation and government spending in the transitional state. ${ }^{27}$

$$
\begin{aligned}
Y_{S}= & \frac{(1-\beta \mu) \sigma}{\Gamma_{\mu \sigma}} r_{S}^{e}+\frac{[(1-\beta \mu)(1-\mu)-\mu \kappa \psi]}{\Gamma_{\mu \sigma}} G_{S}+ \\
& \frac{\mu \kappa \sigma \psi}{\Gamma_{\mu \sigma}} \chi^{I} \tau_{S}^{I}+\frac{(1-\mu)}{\Gamma_{\mu \sigma}}\left\{b\left[(1-\beta \mu)\left(Y_{M}-G_{M}\right)+\sigma \pi_{M}\right]\right\},
\end{aligned}
$$

${ }^{27}$ The relations presented in Propositions 2.1 and 2.2 are obtained by plugging the medium-run levels of output and inflation $\left(Y_{M}, \pi_{M}\right)$ in these intermediate step equations. 


$$
\begin{aligned}
\pi_{S}= & \frac{\kappa \sigma}{\Gamma_{\mu \sigma}} r_{S}^{e}+\frac{(1-\mu)\left(1-\psi \sigma^{-1}\right) \kappa}{\Gamma_{\mu \sigma}} G_{S}+\frac{(1-\mu) \kappa \psi}{\Gamma_{\mu \sigma}} \chi^{I} \tau_{S}^{I}+ \\
& \frac{(1-\mu)}{\Gamma_{\mu \sigma}}\left\{b\left[\kappa\left(Y_{M}-G_{M}\right)+[\beta(1-\mu)+\kappa \sigma] \pi_{M}\right]\right\}
\end{aligned}
$$

where $\Gamma_{\mu \sigma} \equiv(1-\beta \mu)(1-\mu)-\mu \kappa \sigma>0$.

The first line in equations (2-13) and (2-14) represents our benchmark case, where short-run output and inflation are affected by the shock and the fiscal stimulus provided during the crisis $\left(G_{S}\right.$ or $\left.\tau_{S}^{I}\right)$. The second line shows how short-run allocations are affected by medium-run output, inflation and government spending. This is a result of the expectation terms $E_{t} \hat{Y}_{t+1}, E_{t} \pi_{t+1}$ and $E_{t} \hat{G}_{t+1}$ in the $(I S)$ and $(A S)$ equations, (2-1) and (2-2), respectively. Note that the longer the transitional state is expected to last (higher $b$ ), the larger its impact on short-run allocations will be. I should also point out that future inflation is relatively more important than future output in determining shortrun allocations. ${ }^{28}$

In the transitional state, the gap between long- and medium-run interest rates $\left(\bar{r}-i_{M}\right)$ and medium-run fiscal stimulus $\left(G_{M}\right.$ or $\left.\tau_{M}^{I}\right)$ determine output and inflation.

Table 2.9 summarizes the results for short and medium-run output and inflation for all the cases analysed in the Chapter, with stimulus provided either through government spending or income taxes. In those cases in which the monetary authority deviates from rule (2-3) in the transitional state, I assume it keeps the nominal rate at $i_{M}=0$, representing a fully accommodative stance. In those cases for which the solutions depend on the value of probability $b$ (Cases $(C)-(G)$ ), the table presents the solution associated with the maximum value of this probability for which there is a unique determined solution $\left(b_{\max }\right)$. I will discuss how these solutions vary with $b$. In the rest of this Section, I first analyse all the cases assuming the implementation of fiscal stimulus during the crisis through increases in government spending. In what follows, I discuss the stimulus carried out through increases in income taxes. I assume that once a fiscal instrument is chosen during the crisis, the government sticks to it in every following state. This rules out switching between spending and taxes which, although interesting and realistic, complicate unnecessarily the analysis. The list of possible combinations is not exhaustive but allows us to explore the mechanism and understand some appealing results.

\footnotetext{
${ }^{28}$ Under the parametrization assumed, $[\beta(1-\mu)+\kappa \sigma]=0.1041>\kappa=0.0086$.
} 
Chapter 2. Fiscal Stimulus at the Zero Lower Bound: The Role of Expectations and Policy Coordination

Table 2.9: Short- and medium-run output and inflation allocations

\begin{tabular}{rrrrrrrrrrrr}
\hline \hline \multicolumn{1}{c}{$G$ Policy } & \multicolumn{1}{c}{$\tau^{I}$ Policy } \\
\hline \hline & $Y_{S}$ & $\pi_{S}$ & $Y_{M}$ & $\pi_{M}$ & $b_{\max }$ & $Y_{S}$ & $\pi_{S}$ & $Y_{M}$ & $\pi_{M}$ & $b_{\max }$ \\
\hline \hline$(\mathrm{A})$ & -29.9 & -9.9 & & & & -29.9 & -9.9 & & & \\
$(\mathrm{~B})$ & -18.5 & -6.9 & & & & -24.8 & -7.5 & & & \\
$(\mathrm{C})$ & -25.7 & -9.2 & 2.4 & 0.1 & 0.90 & -22.0 & -6.1 & -0.4 & 0.6 & 0.90 \\
$(\mathrm{D})$ & -4.5 & -1.6 & 3.2 & 0.8 & 0.88 & -7.1 & -0.7 & 3.9 & 1.1 & 0.89 \\
$(\mathrm{E})$ & -2.9 & -0.7 & 8.0 & 1.3 & 0.85 & -5.4 & 0.1 & 3.8 & 1.5 & 0.87 \\
$(\mathrm{~F})$ & -37.1 & -12.1 & 2.4 & 0.1 & 0.90 & -27.1 & -8.5 & -0.4 & 0.6 & 0.90 \\
$(\mathrm{G})$ & -3.9 & 0.4 & 9.9 & 2.0 & 0.88 & -6.3 & -0.7 & 4.5 & 1.8 & 0.88 \\
\hline
\end{tabular}

(A) No fiscal or monetary policy

(B) Fiscal stimulus during the crisis state only

(C) Fiscal stimulus kept in the medium run and $i_{M}=r_{t}^{e}+\phi_{\pi} \pi_{t}+\phi_{y} Y_{t}$

(D) Fiscal stimulus in the crisis state only and $i_{M}=0$

(E) Fiscal stimulus kept in the medium run and $i_{M}=0$

(F) Fiscal stimulus implemented after the crisis and $i_{M}=r_{t}^{e}+\phi_{\pi} \pi_{t}+\phi_{y} Y_{t}$

(G) Fiscal stimulus implemented after the crisis and $i_{M}=0$

Note: $b_{\max }$ represents the maximum value of probability $b$ for which Conditions $(C 4)$ and $\left(C 4^{\prime}\right)$ are satisfied. The allocations presented in this table, that depend on the value of $b$, are computed using the respective $b_{\max }$.

\subsection{1}

\section{Impact of Government Spending Stimulus}

In this first part of the analysis, I assume that when a shock hits the economy, there is a $5 \%$ increase in government spending. ${ }^{29} \mathrm{I}$ will show that this stimulus is not sufficient to fully stabilize output and inflation during the crisis $^{30}$, but it is still large enough to reduce the fall in short-run output and inflation significantly. I will not pin down the optimal level of government spending increases in response to the crisis. However, Woordford (33) observes that, in this context, "it is not optimal to fully stabilize inflation and the output gap, despite the feasibility of doing so, because of the inefficient composition of expenditure that this would involve." Since I am focusing the discussion on the comparison of different policies' combinations, the value assumed for the rise in government spending seems reasonable.

In what follows, it becomes clear how the impact of this policy on shortrun output and inflation depends not only on the size of the stimulus provided while the zero lower bound is still binding, but also on what agents expect regarding the interaction between monetary and fiscal policies once the crisis is over. Expectations about the timing of implementation and termination of

\footnotetext{
${ }^{29}$ According to the IMF Fiscal Monitor, this was approximately the increase in government expenditure in the US in 2009.

${ }^{30}$ Conditions $(C 4)$ and $\left(C 4^{\prime}\right)$ are satisfied under this hypothesis.
} 
the fiscal stimulus are also crucial in determining the depth of the recession.

The increase in government spending is expected to be temporary, but it can last longer than the crisis spell. In all the cases, I assume the economy is hit by a shock that makes the zero lower bound binding in the short run. Case $(A)$ represents the fall in output and inflation (30\% and $10 \%$ respectively, according to the parametrization discussed above) that occurs if no stimulus policy is implemented in response to the crisis.

I consider $(B)$ the benchmark case in the analysis. This is the case analyzed in Eggertsson (31), and that is much discussed in the literature. It assumes a perfect timing between the fiscal stimulus and the shock, meaning that the fiscal instrument returns to its pre-crisis level as soon as $r_{t}^{e}$ returns to $\bar{r}$, while the monetary authority goes back to following rule (2-3) right away. This stimulus represents a significant improvement upon the recession caused by the shock (Case $(A)$ ). The fall in output goes from $30 \%$ to $19 \%$, while inflation decreases $7 \%$, instead of $10 \%$.

We note that the termination timing of this stimulus is crucial. If the rise in government spending is kept after the crisis is over, with monetary policy going back to rule (2-3) as soon as $r_{t}^{e}$ returns to $\bar{r}$ (Case $(C)$ ), the decrease in short-run output and inflation will be larger than in Case $(B)$. Figure 2.7 shows how the solutions for short- and medium-run output and inflation depend on the probability associated with the transitional state $(b)$. The longer it is expected to last, i.e., the longer the government spending stimulus is kept after the crisis is over, deeper will be the fall in output and inflation during the crisis. This happens because an increase in government spending in the transitional state, without monetary policy accommodation, has a negative impact on crisis-state output and inflation $\left(\Omega_{Y_{S}, G_{M}}^{T} \leq 0\right.$ and $\Omega_{\pi_{S}, G_{M}}^{T} \leq 0, \forall b \in[0,0.90]$. In this case, keeping the fiscal stimulus, when the zero lower bound is no longer binding, creates a transitional state with higher output and inflation. However, the medium-run output multiplier smaller than one $\left(\Omega_{Y_{M}, G_{M}}^{T} \in[0.90,0.47]^{\dagger}\right)$ implies an increase in activity smaller than the increment in government spending. This makes the term $\left(Y_{M}-G_{M}\right)$ in $(2-13)$ and (2-14) negative and large enough to cancel out the positive effect coming from a higher medium-run inflation. Although getting the timing of returning the fiscal instrument to its pre-crisis level wrong is harmful to the economy, it is still better than not providing any stimulus at all(Case $(A)$ ).

If the transitional state is created by agents' expectations that the monetary authority will keep the nominal interest rate at zero after the crisis is over, with government spending going back to its pre-crisis level as soon as $r_{t}^{e}$ returns to $\bar{r}$ (Case $(D)$ ), the decrease in short-run output and inflation is 
Figure 2.7: Short- and medium-run impact of government spending stimulus
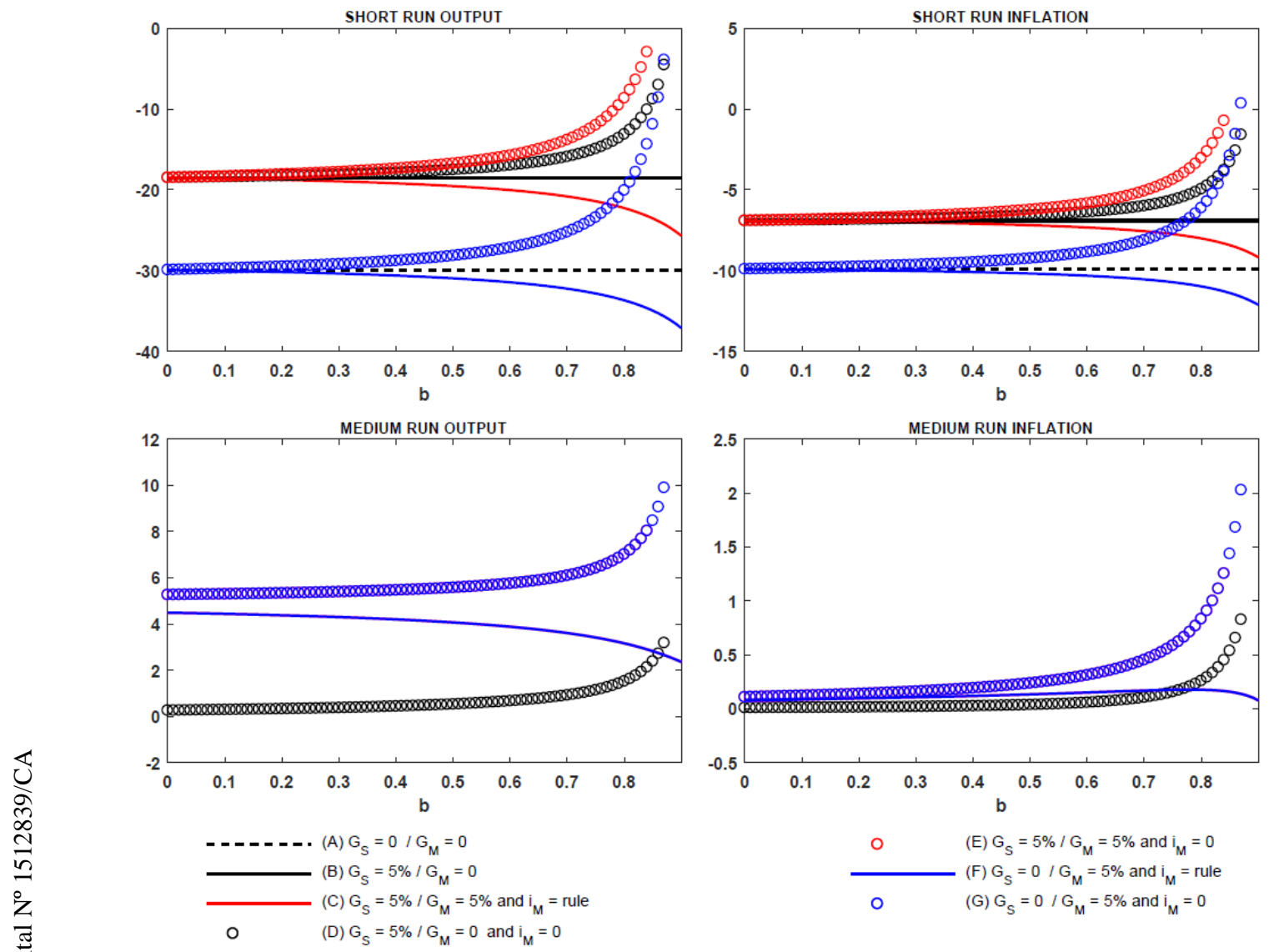

Note: Remember that medium-run allocations do not depend on what happens in the short run. Thus, for medium-run output and inflation, the plots of Cases (C) and (F) coincide, because they have exactly the same policies in the transitional state. The same happens for Cases (E) and (G).

reduced. This effect is stronger for a longer expected transitional state. Keeping the interest rate at $i_{M}=0$ increases output and inflation in the transitional state, with a positive direct effect on short-run output and inflation, as can be seen in equations (2-13) and (2-14). Note that, in this case, the sequence of black circles in Figure 2.7 stops before the highest value assumed for $b=0.9$. This happens because for $b>0.88$ the nominal interest rate implied by rule (2-3) in the short run is positive and the zero lower bound is no longer binding. In fact, for these values of $b$, Condition $(C 4)$ is violated, thus the results from Proposition 2.1 do not apply. Then, I disregard these values.

In the case, where there is an association of the medium-run accommodative monetary policy, with a continuation of the fiscal stimulus after the crisis is over (Case $(E)$ ), short-run output and inflation decrease less than when the monetary authority provides the stimulus in isolation. In this case, Condition $(C 4)$ is no longer satisfied for $b>0.85$. We see that with monetary accommodation of the fiscal stimulus in the transitional state, output and inflation 
increase significantly in the medium run compared to Case $(C)$, where the monetary authority goes back to rule (2-3) as soon as the crisis is over. This happens because monetary accommodation causes the medium-run multipliers to be positive $\left(\Omega_{Y_{M}, G_{M}} \in[1,2.1]\right.$ and $\left.\Omega_{\pi_{M}, G_{M}} \in[0.0,0.1]\right)$, allowing output to increase more than one-for-one with the increase in government spending. Thus, the term $\left(Y_{M}-G_{M}\right)$ in (2-13) and (2-14) will be positive and increasing in $b$, which comes along with higher inflation $\left(\pi_{M}\right)$ as well, undermining the decrease in output and inflation during the crisis.

Regarding the implementation timing of the fiscal stimulus, I analyze an increase in government spending implemented when the zero lower bound is no longer binding. Woordford (33) points out that, even if there is an implementation delay, as long as the eventual increase in government spending occurs during the financial disruption, there will be a positive effect on shortrun output. If most of the stimulus is carried out in a post-crisis environment, without accommodation by the monetary authority, it will be ineffective, or even counter-productive.

I study the extreme case in which the rise in government spending only occurs after the crisis is over. This will also generate a transitional state where the impacts on output and inflation depend on the monetary authority's response. If it goes back to rule (2-3) as soon as $r_{t}^{e}$ returns to $\bar{r}$ (Case $(F)$ ), medium-run output and inflation increase, but the multiplier on output is smaller than one and decreasing in $b\left(\Omega_{Y_{M}, G_{M}}^{T} \in[0.9,0.5]^{\dagger}\right)$. This implies that the term $\left(Y_{M}-G_{M}\right)$ in short-run solution equations (2-13) and (2-14) is negative and significant enough to cancel the positive impact of higher inflation $\left(\pi_{M}\right)$. In this case, we do not have the positive effect of the stimulus provided in the short run, as in Case $(C)$, thus the expectation of an implementation delay makes the economy even worse than if there was no stimulus at all (Case $(A))$.

Nonetheless, if the fiscal stimulus is supposed to be carried out after the crisis is over, but agents also assume it to be associated with a fully accommodative monetary policy $\left(i_{M}=0\right.$ ) (Case $(G)$ ), transitional-state output and inflation increase more than in Case $(F)$. In fact, the monetary accommodation makes the medium-run output multiplier larger than one $\left(\Omega_{Y_{M}, G_{M}} \in[1.0,2.1]\right)$, causing $\left(Y_{M}-G_{M}\right)$ to be positive and increasing in $b$. There is still no stimulus provided in the short run, but there is an improvement upon not providing any stimulus at all (Case $(A))$.

It is interesting to note that, for most values of $b$, delaying the increase in government spending for after the crisis is over, even with monetary policy accommodation, is worse than providing the stimulus only during the crisis. 
Nevertheless, when the transitional state is expected to be long (higher $b$ ), the decrease in short-run output (for $b \geq 0.83$ ) and inflation (for $b \geq 0.79$ ) can get smaller than in the benchmark (Case $(B)$ ). They are close to those observed when there is a transitional state generated by an accommodative policy with fiscal stimulus only during the crisis (Case $(D)$ ). The key for this result is that with a more extended transitional state, medium-run inflation is expected to be high, which helps to reduce the depth of the recession.

Summarizing, the timing of implementation of a fiscal stimulus through increases in government spending, and expectations regarding its termination, are fundamental in determining the depth of the recession caused by a shock that makes the zero lower bound binding. It is crucial to the fiscal authority to be able to respond quickly when the shock hits the economy. But it is also essential for both monetary and fiscal authorities to coordinate their policies after the crisis is over and, above all, to be able to signal them correctly, so agents incorporate this into their expectations, helping to mitigate the decrease observed in output and inflation during the crisis.

\subsection{2}

\section{Impact of Income Taxes Stimulus}

I repeat the discussion using income taxes as the fiscal instrument to stimulate the economy, also assuming a 5\% increase in taxes during the crisis. ${ }^{31}$ Table 2.9 summarizes the results for short- and medium-run output and inflation. In a nutshell, an increase in income taxes is stimulative during the crisis and it continues boosting the economy as long as monetary policy keeps being accommodative. Besides, an implementation delay is not as harmful as in the case of government spending stimulus, and it can still be beneficial if expected to be associated with an accommodative monetary policy.

Figure 2.8 presents short- and medium-run output and inflation for Cases $(A)-(G)$. As discussed above regarding the multipliers, an increase in taxes during the crisis (Case $(B)$ ) has a positive effect, reducing the fall in output to $25 \%$ and inflation to $7.5 \%$. We observe that this stimulus is not as powerful as the one provided by government spending, but it helps to reduce the depth of the recession. However, in contrast to government spending, if the fiscal authority temporarily keeps income taxes higher after the crisis is over (Case $(C)$ ), short-run output and inflation will decrease less than if the timing was perfect relative to the crisis. In this case, the recession is smaller the longer

${ }^{31}$ In fact, according to the Fiscal Monitor, 2009 data for the US shows there was a fall of $2 \%$ in government revenues as a percent of GDP. We assume a $5 \%$ increase in income taxes to make the results comparable to those obtained with government spending stimulus and discuss the impact of a positive short-run output multiplier for income taxes. 
Figure 2.8: Short-Run Impact of Temporary Income Tax Stimulus
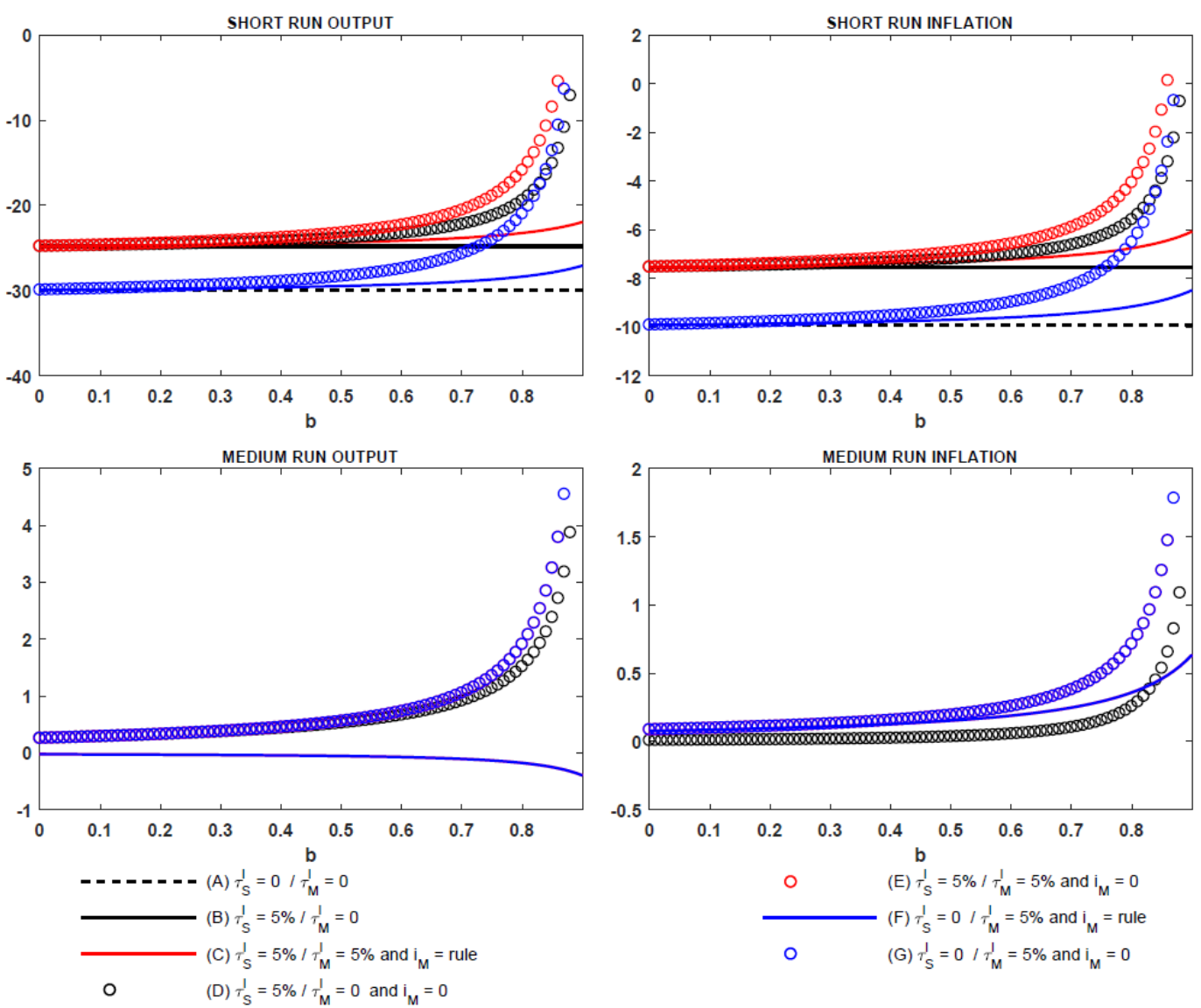

Note: Remember that medium-run allocations do not depend on what happens in the short run. Thus, for medium-run output and inflation, the plots of Cases (C) and (F) coincide, because they have exactly the same policies in the transitional state. The same happens for Cases (E) and (G).

the transitional state is expected to last (higher $b$ ). To understand this result, I analyze the transitional state created by keeping income taxes higher after the crisis is over. Given that monetary policy returns to rule (2-3) as soon as $r_{t}^{e}$ returns to $\bar{r}$, medium-run output decreases $\left(\Omega_{Y_{M}, \tau_{M}^{I}}^{T} \in[0.0,-0.1]^{\dagger}\right)$, while inflation increases $\left(\Omega_{Y_{M}, \tau_{M}^{I}}^{T} \in[0.004,0.003]\right)$. Using equations (2-13) and (2-14), we see that medium-run inflation have more weight than output in determining the short-run allocations. Therefore, even with an expected small decrease in activity after the crisis is over, the fact that this policy generates expectations of higher inflation when the zero lower bound is no longer binding stimulates the economy in the short run.

Instead, if the fiscal stimulus is dropped as soon as the crisis is over, but monetary policy can stimulate the economy keeping the transitional-state nominal interest rate at $i_{M}=0$ (Case $(D)$ ), the longer the transitional state generated by this policy is expected to last, smaller will be the fall in output and inflation. In the transitional state, keeping the nominal interest rate at zero 
increases output and inflation through the positive impact of the gap between steady-state and medium-run nominal interest rate $\left(\bar{r}-i_{M}\right)$ (See Table 2.2). Under the expectation of both output and inflation being higher after the crisis is over, the economy gets a boost in the short run.

The recession will be further mitigated if the government associates a medium-run accommodative monetary policy with a continuation of the fiscal stimulus (Case $(E)$ ). Besides getting the effect of the positive interest rate gap $\left(\bar{r}-i_{M}\right)$, this policy extends the period with a positive output and inflation multiplier $\left(\Omega_{Y_{M}, \tau_{M}^{I}} \in[0,0.9]\right.$ and $\Omega_{Y_{M}, \tau_{M}^{I}} \in[0,0.1]$, respectively), boosting the economy in the transitional state. The stimulative effect of higher income taxes after the crisis is over is exacerbated by the accommodative monetary policy, further mitigating the decrease in short-run output and inflation.

I also analyse what happens if the effective rise in taxes is expected to be delayed, with its implementation only occurring when the zero lower bound is no longer binding (Cases $(F)$ and $(G)$ ). In the case of taxes, this is even more likely than with government spending, which is usually able to respond quickly. Again, the impact depends on how the monetary authority responds to this policy. If it goes back to the rule (2-3) as soon as the crisis is over (Case $(F)$ ), it can still undermine the depth of the recession because, although it decreases output in the medium run, it is able to create expectations of higher inflation after $r_{t}^{e}$ returns to $\bar{r}$. Even though the decreases in short-run output and inflation are larger than if the policy was implemented during the crisis (Case $(B))$, it is still better than not implementing any policy at all (Case $(A))$. This contrasts with what happens under a government spending stimulus where the delayed policy, under a non-accommodative monetary policy, is worse than a total absence of fiscal policy.

However, associating the delayed rise in income taxes with a fully accommodative monetary policy $\left(i_{M}=0\right)$ (Case $(G)$ ) increase medium-run output and inflation. For low values of $b$, it is still worse than the timely implementation because it does not count with the stimulative effect of the policy in the short run. But, for $b \geq 0.74$, the fall in output is smaller than in the benchmark case. The same happens to inflation for $b \geq 0.77$. We observe that short-run allocations get close to those found in Cases $(D)$ and $(E)$ for levels of $b$ close to the top of the interval.

To sum up, besides being stimulative during the crisis, an increase in income taxes that continues once the zero lower bound is no longer binding can boost the economy more, especially under an accommodative monetary policy. Even if there is an implementation delay, it is still better than not providing any policy at all, particularly under monetary policy accommodation with the 
transitional state expected to last longer. Therefore, independently of the fiscal instrument used to stimulate the economy, it is crucial for monetary and fiscal authorities to be able to coordinate and correctly signal their policies, not only during the crisis, but in future periods as well.

\section{7}

\section{Conclusion}

This Chapter discussed the crucial role expectations regarding future policies play in determining the depth of a crisis produced by a shock that causes the nominal interest rate to reach the zero lower bound. It showed that when analysing the impact of a fiscal stimulus provided during the crisis, we need to go beyond looking at short-run multipliers. When the stimulus is expected to be implemented and terminated, and how it is expected to be accommodated by the monetary authority when the zero lower bound ceases to bind, is determinant to evaluate its effects on output and inflation during the crisis.

In such a severe downturn, monetary and fiscal policies will be more expansionary if they last longer than the shock spell and coordinate their moves. This coordination is necessary not only during the crisis, but also regarding the commitment to future policies. Another crucial aspect for stimulating the economy is that the fiscal authority needs to be able to respond quickly to the shock, minimizing implementation delays and correctly signalling the duration of the stimulus.

The most successful combinations of policies are those in which the fiscal stimulus is expected to be carried out with minimum delay once the crisis hits the economy. If kept when the shock is no longer causing the zero lower bound to bind, the fiscal policy only improves the crisis state output and inflation, if expected to be associated with an accommodative monetary policy. This combination of policies can create expectations of higher inflation when the crisis is over and, even if small decreases in output are expected, generate positive impacts on economic activity during the crisis.

I also obtained that an increase in income taxes stimulates the economy when the zero lower bound is binding. However, this instrument's impacts are smaller than those obtained with a stimulus provided through increases in government spending. Besides, keeping higher income taxes after the crisis is over, even without monetary policy accommodation, contributes to improving the economy, in contrast to what we observe with government spending. This means that a mistake in ending the stimulus implemented through increases in income taxes is not as harmful as the one in the timing of reverting an 
increase in government spending. This is also valid for the implementation timing of policies. If the stimulus is carried out through increases in government spending that are expected to occur only when the crisis is over, without any accommodation from monetary policy, it is better not to implement any policy at all. Delaying the increase in taxes until the shock is no longer causing the zero lower bound to bind can still reduce the depth of the crisis. In this case, it is even better than not implementing any policy at all.

A critical aspect of the analysis is that the trustworthy coordination between monetary and fiscal policy authorities is crucial in determining the outcome of expectations regarding future policies. It is important to highlight that I assumed agents to believe that both authorities can commit to the announced policies, once the zero lower bound is no longer binding. I did not discuss time consistency issues, but understand that monetary and fiscal authorities may not have the incentive to stick to stimulative policies once the crisis is over, especially under such rare event which is not expected to occur again in a considerable time horizon.

Another explicit limitation of the analysis presented is that it does not account for the effects of these policies on debt dynamics. It would be interesting to relax the Ricardian equivalence hypothesis and discuss how expected future fiscal policy adjustments, necessary to balance the government budget constraint, would impact the crisis-state allocations.

Other avenues for future research should include investigating how much the results depend on the structure of the model and the parameters adopted in the calibration, enriching the discussion of other fiscal policy instruments and improving the probability structure of the model to account for the possibility of the economy hitting the zero lower bound again in future periods, besides allowing for a longer crisis spell.

The analysis of this Chapter discusses the impact of various combinations of fiscal and monetary actions on the levels of output and inflation during the crisis. One might ask what would be the best policy combination in this environment. This is the focus of next Chapter, which examines the impacts of these policy combinations in terms of welfare. 


\section{3 \\ Fiscal Stimulus at the Zero Lower Bound: Welfare Analysis and Optimal Transitional State Monetary Policy}

\section{1 \\ Introduction}

In the previous Chapter, I examined the impact of expectations with respect to future monetary and fiscal policies on the levels of inflation and output during a crisis caused by a shock that makes the nominal interest rate hit its effective lower bound. By allowing monetary and fiscal authorities to keep using their instruments after the crisis is over, I studied a set of policy combinations that could amplify or undermine the depth of the recession.

In general, policies are chosen to reduce the welfare losses associated with output and inflation volatilities. Nevertheless, in an environment where the zero lower bound for the nominal interest rate is binding, while inflation and output are down, one of the goals of policy actions should be to create expectations of higher inflation in the future.

Given the interplay between the policies adopted and their impact on the economy, a naturally raised question is how we can compare the impacts of monetary and fiscal authorities' possible actions in terms of welfare losses. This Chapter aims at discussing this question. I also study what would be the optimal level for the nominal interest rate that the monetary authority should set in the transitional state if it wants to minimize the losses in welfare associated with the crisis. This optimal level depends on whether the fiscal authority would also keep providing some type of stimulus in the transitional state or not.

Following Woodford (2), I derive the microfounded welfare loss function implied by the households' utility function in the model. Given the probability structure of the model, it is possible to obtain a welfare loss function in present discounted value terms, which allows ranking the various combinations of policies adopted in each state. With this function, I can discuss the impact of policies not only regarding their effects on output and inflation during the crisis, which has been the focus of most contributions in the literature, but also considering their impact in future periods as well. 
The derivation of the welfare loss function in present discounted value terms also permits analysing the optimal choice for the monetary policy instrument once the shock that caused the zero lower bound to bind is no longer effective. I obtain that, after the crisis is over, it is optimal for the monetary authority to keep the nominal interest rate at zero for a few periods. However, if the transitional state created by this policy is expected to have a long spell, the optimal level for the nominal interest rate becomes positive.

I examine the implications for output and inflation during the crisis and the welfare losses implied by this optimal monetary policy in the transitional state, associated with one of the fiscal instruments or not. I compare this with the policy combinations analysed in Chapter 2.

The next Section presents the derivation of the welfare loss function in present discounted value terms. Section 3.3 shows how the optimal level for the transitional state monetary policy is obtained. Section 3.4 discusses the impacts in terms of welfare of the set of policy combinations considered in Chapter 2 and those with the optimal monetary policy, considering the use of both fiscal instruments, government spending and income taxes. Section 3.5 concludes the Chapter.

\section{2}

\section{Welfare Loss Function}

According to Woodford (2), there is an agreement in the economic literature with respect to the general form of the objective function of the monetary authority, which should target a low and stable level of inflation, but should also be concerned with the stability of economic activity. He argues that "a desirable monetary policy is one that achieves a low expected value of a discounted loss function, where the losses each period are weighted averages of terms quadratic in the deviation of inflation from a target rate and in some measure of output relative to potential." (Chapter 6, pg.381).

The relative weights of inflation and output in the loss function were set adhocly in contributions like Clarida et. al. (43). However, Woodford (2) shows that this loss function can be derived from microfoundations of the model, with the weights associated with inflation and output determined by the model's parameters. He points out that private agents' preferences provide a natural welfare criterion, thus the loss function is obtained from the household's utility function considered in the model. If one assumes that households' care about the level of government spending, some weight will be put to it in the loss function as well. This is the case of the loss function used in Woodford (33) and Eggertsson (31). 
In order to obtain the exact expression for the weights in the loss function, I derive it from the households' utility function. ${ }^{1}$ Then, using the probability structure of the model in Chapter 2, I obtain the expression for the loss function in present discounted value terms. ${ }^{2}$ This is presented in Proposition 3.1 below.

Proposition 3.1 The welfare loss function in this model is given by

$$
L_{t} \equiv \sum_{t=0}^{\infty} \beta^{t}\left\{\pi_{t}^{2}+\lambda_{y}\left(\hat{Y}_{t}-\Gamma \hat{G}_{t}\right)^{2}+\lambda_{g} \hat{G}_{t}^{2}\right\}
$$

where $\Gamma \equiv \frac{\sigma^{-1} \gamma}{\sigma^{-1}+\omega}, \lambda_{y} \equiv \frac{\kappa}{\theta}$ and $\lambda_{g} \equiv \lambda_{y} \Gamma(1-\gamma-\Gamma)$. Given the probability structure of the model, this function can be expressed in present discounted terms as

$$
L^{P D V}=\left\{\begin{array}{l}
\frac{1}{1-\beta \mu}\left(\pi_{S}^{2}+\lambda_{y}\left(Y_{S}-\Gamma G_{S}\right)^{2}+\lambda_{g} G_{S}^{2}\right)+ \\
\frac{\beta(1-\mu) b}{(1-\beta \mu)(1-\beta b)}\left(\pi_{M}^{2}+\lambda_{y}\left(Y_{M}-\Gamma G_{M}\right)^{2}+\lambda_{g} G_{M}^{2}\right)+ \\
\frac{\beta(1-\mu)(1-b)}{(1-\beta)(1-\beta \mu)(1-\beta b)}\left(\pi_{L}^{2}+\lambda_{y}\left(Y_{L}-\Gamma G_{L}\right)^{2}+\lambda_{g} G_{L}^{2}\right)
\end{array}\right\} .
$$

Proof. See Appendix C.1.

Chapter 2 showed that different policies' combinations have very different implications for the levels of inflation and output in each state. The welfare loss function in equation (3-2) makes this comparison between policy alternatives very straightforward. Besides depending on the households' discount factor $(\beta)$, this function also depends on the duration of the crisis $(\mu)$ and of the transitional state $(b)$. Note that since I assume policies to be temporary, in the long run $Y_{L}=\pi_{L}=G_{L}=0$, so the last line in (3-2) disappears. Also, if both monetary and fiscal authorities go back to their long run policies $\left(G_{t}=\tau_{t}=0\right.$ and the nominal interest rate given by the rule (2-3) right after the crisis is over, there will be no transitional state and the second line in (3-2) disappears as well.

Another characteristic of the welfare function obtained in Proposition 3.1 worth highlighting is the much higher weight it puts in the stabilization of inflation. ${ }^{3}$ As pointed out by Woodford (2), this is the result of the degree of

\footnotetext{
${ }^{1}$ The functional form and other characteristics of the model are discussed in the proof of Proposition 3.1 in Appendix C.1.

${ }^{2}$ This present discounted loss function is derived in the most general form, allowing for government spending in every state and including output and inflation in the long run.

${ }^{3}$ While the weight on $\pi$ is 1 , under the parameter values used in Chapter 2, the weight on output is $\lambda_{y}=6.72 e-04$ and the weight on government spending is $\lambda_{g}=4.1 e-05$.
} 
nominal rigidity present in the model and usually contrasts with the weights set adhocly.

The welfare loss function derived here is analogous to that presented by Woodford (33), with slight differences in the expressions for the weights due to the specification of the model. He uses this function to discuss the optimal level of government spending during the crisis. However, since in his set-up there is no transitional state, the economy goes back to the steady state $\left(Y_{L}=\pi_{L}=0\right)$ right after the crisis is over. Thus, the present discounted value of his loss function corresponds only to the first line in equation (3-2). I will not discuss the optimal level for fiscal instruments here. They are set at a given level throughout the analysis. I use the present discounted value of the welfare loss function to obtain the optimal transitional-state interest rate below and to compare the welfare implications of different policies' combinations.

\section{3}

\section{Optimal Transitional State Monetary Policy}

I use the welfare loss function derived in Proposition 3.1 as the objective function of an optimization problem that searches for the optimal level of the monetary policy instrument in the transitional state. This problem looks for the medium-run nominal interest rate that minimizes the welfare losses restricted to the equilibrium allocations for output and inflation in each state and to a non-negative rate. The allocations for output and inflation are those obtained in Proposition 2.1 in Chapter 2. The solution for this problem is presented in the following proposition.

Proposition 3.2 Assuming that Conditions $(C 1)-(C 4)$ from Proposition 2.1 hold, if the monetary authority keeps the nominal interest rate fixed at an optimal level $i_{M}^{*}$ in the transitional state, it picks this level by solving the following minimization problem

$$
\begin{aligned}
& \min _{\left\{i_{M}\right\}} L^{P D V} \\
& \text { s.t. } Y_{S}, \pi_{S}, Y_{M}, \pi_{M}, Y_{L}, \pi_{L} \\
& i_{M} \geq 0
\end{aligned}
$$

where $L^{P D V}$ is defined in equation (3-2) and the levels of output and inflation in each state are provided by equations (2-4) - (2-7) in Proposition 2.1. The solution to this problem yields an optimal nominal interest rate given by

$$
i_{M}^{*}=\left\{\begin{aligned}
i_{M}^{o p t} & , \text { if } i_{M}^{o p t}>0 \\
0 & , \text { otherwise }
\end{aligned}\right.
$$


$i_{M}^{o p t}=\bar{r}+\frac{1}{\Omega_{i_{M}, \bar{r}}^{*}}\left\{\Omega_{i_{M}, r_{S}^{e}}^{*} r_{S}^{e}+\Omega_{i_{M}, G_{S}}^{*} G_{S}+\Omega_{i_{M}, \tau_{S}^{I}}^{*} \tau_{S}^{I}+\Omega_{i_{M}, G_{M}}^{*} G_{M}+\Omega_{i_{M}, \tau_{M}^{I}}^{*} \tau_{M}^{I}\right\}$,

where the analytical expressions for the coefficients $\Omega_{i_{M}, j}^{*}, j \stackrel{(3-3)}{\in}$ $\left\{\bar{r}, r_{S}^{e}, G_{S}, \tau_{S}^{I}, G_{M}, \tau_{M}^{I}\right\}$ are defined in the appendix and depend on the structural parameters and the coefficients $\left(\Omega^{\prime} s\right)$ from Proposition 2.1.

Proof. See Appendix C.2.

Equation (3-3) and Table 3.1 postulate that the optimal nominal interest rate depends on the size of the shock $\left(r_{S}^{e}\right)$, the expected duration of the financial disturbance $(\mu)$ and of the transitional state $(b)$. It is interesting to note that $i_{M}^{o p t}$ depend not only on the fiscal instruments used in the transitional state, but on those used in the crisis state as well.

Table 3.1: Analytical expressions for optimal nominal interest rate coefficients - Proposition 3.2

\begin{tabular}{|c|c|}
\hline$\Omega_{i_{M}, \bar{r}}^{*}=[$ & $\begin{array}{l}\left(\Omega_{\pi_{S}, i_{M}}\right)^{2}+\lambda_{y}\left(\Omega_{Y_{S}, i_{M}}\right)^{2}+ \\
\frac{\beta(1-\mu) b}{(1-\beta b)}\left(\left(\Omega_{\pi_{M}, i_{M}}\right)^{2}+\lambda_{y}\left(\Omega_{Y_{M}, i_{M}}\right)^{2}\right)\end{array}$ \\
\hline \multicolumn{2}{|c|}{$\Omega_{i_{M}, r_{S}^{e}}^{*}=\left[\Omega_{\pi_{S}, i_{M}} \Omega_{\pi_{S}, r_{S}^{e}}+\lambda_{y} \Omega_{Y_{S}, i_{M}} \Omega_{Y_{S}, r_{S}^{e}}\right]$} \\
\hline \multicolumn{2}{|c|}{$\Omega_{i_{M}, G_{S}}^{*}=\left[\Omega_{\pi_{S}, i_{M}} \Omega_{\pi_{S}, G_{S}}+\lambda_{y} \Omega_{Y_{S}, i_{M}}\left(\Omega_{Y_{S}, G_{S}}-\Gamma\right)\right]$} \\
\hline \multicolumn{2}{|c|}{$\Omega_{i_{M}, \tau_{S}^{I}}^{*}=\left[\Omega_{\pi_{S}, i_{M}} \Omega_{\pi_{S}, \tau_{S}^{I}}+\lambda_{y} \Omega_{Y_{S}, i_{M}} \Omega_{Y_{S}, \tau_{S}^{I}}\right]$} \\
\hline$\Omega_{i_{M}, G_{M}}^{*}=$ & {$\left[\begin{array}{l}\Omega_{\pi_{S}, i_{M}} \Omega_{\pi_{S}, G_{M}}+\lambda_{y} \Omega_{Y_{S}, i_{M}} \Omega_{Y_{S}, G_{M}}+ \\
\frac{\beta(1-\mu) b}{(1-\beta b)}\left(\Omega_{\pi_{M}, i_{M}} \Omega_{\pi_{M}, G_{M}}+\lambda_{y} \Omega_{Y_{M}, i_{M}}\left(\Omega_{Y_{M}, G_{M}}-\Gamma\right)\right)\end{array}\right.$} \\
\hline$\Omega_{i_{M}, \tau_{M}^{I}}^{*}=$ & {$\left[\begin{array}{l}\Omega_{\pi_{S}, i_{M}} \Omega_{\pi_{S}, \tau_{M}^{I}}+\lambda_{y} \Omega_{Y_{S}, i_{M}} \Omega_{Y_{S}, \tau_{M}^{I}}+ \\
\frac{\beta(1-\mu) b}{(1-\beta b)}\left(\Omega_{\pi_{M}, i_{M}} \Omega_{\pi_{M}, \tau_{M}^{I}}+\lambda_{y} \Omega_{Y_{M}, i_{M}} \Omega_{Y_{M}, \tau_{M}^{I}}\right)\end{array}\right.$} \\
\hline
\end{tabular}

The coefficients $\Omega_{i_{M}, j}^{*}, j \in\left\{\bar{r}, r_{S}^{e}, G_{S}, \tau_{S}^{I}, G_{M}, \tau_{M}^{I}\right\}$ are combinations of short- and medium-run output and inflation multipliers obtained in Proposition 2.1. Recall that the analytical expressions for these multipliers are presented in Tables $2.2-2.3$.

One important point to highlight in Proposition 3.2 is that the optimal nominal interest rate in the transitional state (3-3) is obtained under the hypothesis that the zero lower bound is binding in the short run (Condition $(C 4)$ ). This Condition depends on how long the transitional state is expected to last. As we will see in the exercises in the next Section, under the parametrization chosen, very high values of $(b)$ violate Condition $(C 4)$, making the results in Propositions 2.1 and 3.2 no longer valid. In the next Section I 
discuss the implications of this optimal transitional state monetary policy and compare its impacts in terms of welfare with those of the policy combinations discussed in Chapter 2.

\section{4}

\section{Welfare Analysis of Monetary and Fiscal Policies Combinations}

Chapter 2 analysed the impact of combinations of monetary and fiscal policies adopted during the crisis, and expected for when the crisis is over, on output and inflation. Most combinations reduce the depth of the crisis, but one might ask which of them would make agents better off in terms of welfare.

This Section examines the welfare implications of Cases $(A)$ to $(G)$ discussed in Chapter 2, and add three more cases to allow the analysis of the optimal transitional state monetary policy. In these cases, this optimal monetary policy is combined with the possible actions of the fiscal authority: (i) Fiscal stimulus implemented only during the crisis (Case $(H)$ ); $(i i)$ Fiscal stimulus implemented during the crisis and kept in the transitional state (Case $(I))$; (iii) Fiscal stimulus implemented only after the crisis is over (Case $(J)$ ). In these three cases, for most values of the probability associated with the transitional state $(b)$, equation (3-3) implies $i_{M}^{\text {opt }}<0$, thus the medium-run optimal monetary policy is to keep the nominal interest rate at $i_{M}^{*}=0$, independently of the fiscal instruments used or when it is implemented. Only very high values of $(b)$ imply a positive nominal interest rate. Hence, for most expected durations of the transitional state, Cases $(H),(I)$ and $(J)$ coincide with Cases $(D),(E)$ and $(G)$, respectively.

Table 3.2 extends the results of Table 2.9 to account for cases $(H)-(J)$ and to present the welfare losses associated with each policy combination analysed, both when the fiscal authority implements its policy through increases in government spending or in income taxes. 
Table 3.2: Short- and medium-run output and inflation allocations and welfare losses

\begin{tabular}{|c|c|c|c|c|c|c|c|c|c|c|c|c|}
\hline & \multicolumn{6}{|c|}{$G$ Policy } & \multicolumn{6}{|c|}{$\tau^{I}$ Policy } \\
\hline & $Y_{S}$ & $\pi_{S}$ & $Y_{M}$ & $\pi_{M}$ & $\begin{array}{c}\text { Welfare } \\
\text { Losses }\end{array}$ & $b_{\max }$ & $Y_{S}$ & $\pi_{S}$ & $Y_{M}$ & $\pi_{M}$ & $\begin{array}{r}\text { Welfare } \\
\text { Losses }\end{array}$ & $b_{\max }$ \\
\hline$(\mathrm{A})$ & -29.9 & -9.9 & & & 9.9 & & -29.9 & -9.9 & & & 9.9 & \\
\hline (B) & -18.5 & -6.9 & & & 4.8 & & -24.8 & -7.5 & & & 5.7 & \\
\hline (C) & -25.7 & -9.2 & 2.4 & 0.1 & 8.5 & 0.90 & -22.0 & -6.1 & -0.4 & 0.6 & 3.8 & 0.90 \\
\hline (D) & -4.5 & -1.6 & 3.2 & 0.8 & 0.3 & 0.88 & -7.1 & -0.7 & 3.9 & 1.1 & 0.1 & 0.89 \\
\hline$(\mathrm{E})$ & -2.9 & -0.7 & 8.0 & 1.3 & 0.1 & 0.85 & -5.4 & 0.1 & 3.8 & 1.5 & 0.1 & 0.87 \\
\hline$(\mathrm{F})$ & -37.1 & -12.1 & 2.4 & 0.1 & 14.8 & 0.90 & -27.1 & -8.5 & -0.4 & 0.6 & 7.3 & 0.90 \\
\hline$(\mathrm{G})$ & -3.9 & 0.4 & 9.9 & 2.0 & 0.3 & 0.88 & -6.3 & -0.7 & 4.5 & 1.8 & 0.3 & 0.88 \\
\hline$(\mathrm{H})$ & -4.5 & -1.6 & 3.2 & 0.8 & 0.3 & 0.88 & -5.7 & 0.0 & 3.8 & 1.3 & 0.1 & 0.90 \\
\hline$(\mathrm{I})$ & -2.9 & -0.7 & 8.0 & 1.3 & 0.1 & 0.85 & -6.7 & 0.1 & 2.7 & 1.7 & 0.2 & 0.90 \\
\hline$(\mathrm{J})$ & -6.0 & -0.2 & 8.7 & 2.2 & 0.4 & 0.90 & -5.7 & -0.2 & 3.9 & 2.1 & 0.4 & 0.90 \\
\hline
\end{tabular}

(A) No fiscal or monetary policy

(B) Fiscal stimulus during the crisis state only

(C) Fiscal stimulus kept in the medium run and $i_{M}=r_{t}^{e}+\phi_{\pi} \pi_{t}+\phi_{y} Y_{t}$

(D) Fiscal stimulus in the crisis state only and $i_{M}=0$

(E) Fiscal stimulus kept in the medium run and $i_{M}=0$

(F) Fiscal stimulus implemented after the crisis and $i_{M}=r_{t}^{e}+\phi_{\pi} \pi_{t}+\phi_{y} Y_{t}$

(G) Fiscal stimulus implemented after the crisis and $i_{M}=0$

(H) Fiscal stimulus in the crisis state only and $i_{M}=i_{M}^{*}$

(I) Fiscal stimulus kept in the medium run and $i_{M}=i_{M}^{*}$

(J) Fiscal stimulus implemented after the crisis and $i_{M}=i_{M}^{*}$

Note: $b_{\max }$ represents the maximum value of probability $b$ for which there is a determined solution in the respective case, i.e. those for which Condition $(C 4)$ in Proposition 2.1 is not violated. The output and inflation allocations and the welfare losses presented in this table, that depend on the value of $b$, are computed using the respective 
Note that the results presented in this table are those with the maximum value of the probability associated with the transitional state for which Condition $(C 4)$ is not violated. When government spending is the instrument used in fiscal policy, this maximum value is the same in Cases $(D)$ and $(H)$ and in $(E)$ and $(I)$. This does not happen for Cases $(G)$ and $(J)$, and for the cases in which income taxes are the instrument used by the fiscal authority. I discuss the results with each fiscal instrument separately in what follows.

\subsection{1}

\section{Impact of Government Spending Stimulus}

The previous Chapter showed that the choices of monetary and fiscal policies implemented during the crisis and expected to be implemented once it is over, are able to amplify or undermine the decreases in output and inflation caused by the shock that made the zero lower bound binding. However, the combinations of these policies have different impacts for economic activity and for the level of prices in the crisis and transitional states. So I ask how the equilibrium implied by each policy combination impacts welfare losses.

The longer the crisis is expected to last (higher $\mu$ ), bigger will be the decreases in output and inflation caused by the shock. Under the calibration used $(\mu=0.904)$, Table 3.2 shows that if there is no fiscal stimulus either in the short or in the medium run, and no expectation of an accommodative monetary policy adopted after the crisis (Case $(A)$ ), there is an expressive fall in short-run output and inflation (-29.9 and -9.9 , respectively), which imply in significant welfare losses (9.9).

A fiscal stimulus implemented through increases in government spending only during the crisis (Case $(B))$ is able to reduce the falls in output and inflation ( -18.9 and -6.9 , respectively) reducing welfare losses as well (4.8). If the stimulus provided during the crisis is kept for a few periods once it is over, without any accommodation from the monetary authority, which goes back to following the rule (2-3) (Case $(C)$ ), although not reducing the drops in shortrun output and inflation as in (Case $(B)$ ), it is still able to reduce welfare losses when compared with Case $(A)$. However, it does not reduce as much as in the case in which the fiscal stimulus is perfectly timed to be terminated when the zero lower bound is no longer binding (Case $(B)$ ). In fact, Figure 3.1 shows that in (Case $(C)$ ), the longer the fiscal stimulus is expected to be kept after the crisis is over (higher $b$ ), larger is the present discounted value of welfare losses. Nevertheless, if the continuation of the fiscal stimulus is combined with a fully accommodative monetary policy in the transitional state (Cases $(E)$ and $(I)$ ), it significantly reduces the falls in output and inflation and the associated 
welfare losses. In fact, for a given value of the probability $b$ these are the cases that imply the lowest welfare losses. Note in the bottom left plot of Figure 3.1 that $i_{M}^{*}=0$ for all possible values of $b$ in Case $(I)$. But both in this case, and in Case $(E)$, Condition $(C 4)$ of Proposition 2.1 is not satisfied for $b \geq 0.85$, which makes the zero lower bound no longer binding during the crisis and the results from Proposition 2.1 do not apply.

Figure 3.1: Welfare losses and optimal transitional state monetary policy with government spending stimulus
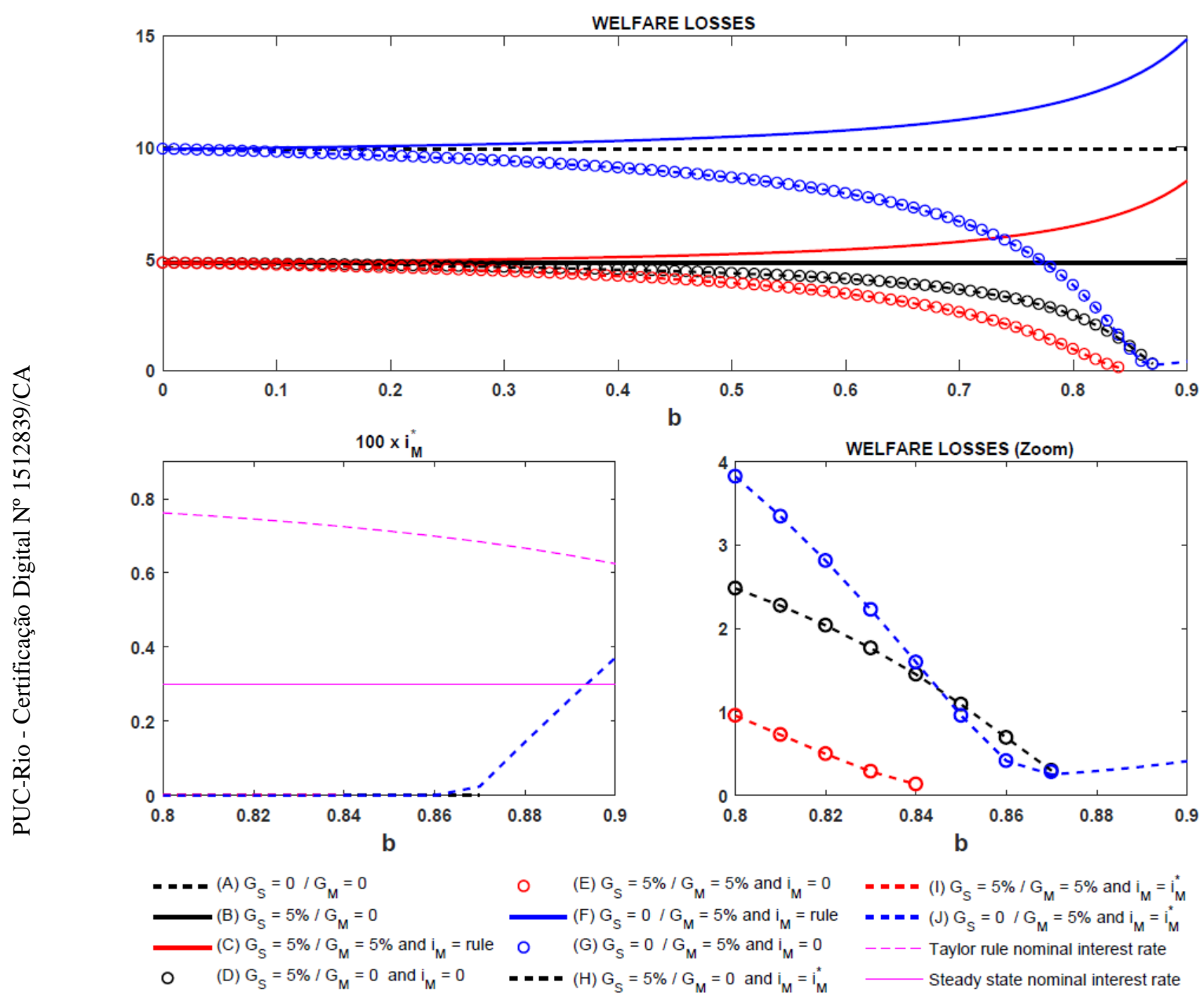

Notes:

(i) The top plot displays welfare losses associated with each case as a function of probability $b$.

(ii) The bottom left plot presents the optimal level for the nominal interest rate in the transitional state $\left(i_{M}^{*}\right)$. It only plots the values for $b \in[0.80,0.90]$ because lowers values of $b$ imply $i_{M}^{*}=0$. Only very high values of $b$ imply $i_{M}^{*}>0$. This plot includes the steady-state nominal interest rate $(\bar{r})$ and its level implied by the rule (2-3) under Proposition 3.2.

(iii) The bottom right plot shows a 'zoomed' view of the bottom right part of the top plot. It highlights the behaviour of welfare losses when $i_{M}^{*}>0$ is allowed.

(iv) The plots that are discontinued before $b=0.90$ violate Condition (C4) from Proposition 2.1 , thus the ZLB is no longer binding for these higher values of $b$. This happens for Cases $(D),(E),(G),(H)$ and $(I)$.

(v) In the bottom left plot, Case $(I)$ is 'hidden' since it implies $i_{M}^{*}=0$ for all values of $b$ for which the ZLB is still binding. 
It is interesting to note that an accommodative monetary policy during the transitional state $\left(i_{M}=i_{M}^{*}=0\right)$, even if not associated with the continuation of the fiscal stimulus (Cases $(D)$ and $(H)$ ), is also able to reduce welfare losses when compared to Case $(B)$, but not as much as in Cases $(E)$ and $(I)$. In Cases $(D)$ and $(H)$, Condition $(C 4)$ is no longer satisfied for $b>0.88$.

An important issue for the fiscal authority is the possibility that it faces delays to implement its policy after the economy enters the crisis state. In the cases discussed so far, I assumed that if the fiscal stimulus is used in the short run, it is implemented as soon as the crisis start. But I also analyse the extreme possibility that there is such a big delay that the policy is only implemented once the crisis is over. If there is no accommodation from monetary policy (Case $(F)$ ), it implies welfare losses that are larger than if no policy was implemented at all (Case $(A))$ and the longer the fiscal stimulus is expected to be kept, worse off is the economy. Nevertheless, if this delay is expected to be accompanied by an accommodative monetary policy (Cases $(G)$ and $(J)$ ), it can significantly reduce welfare losses and get even below that from the benchmark case $(B)$ if expected to be long enough (high values of $b$ ).

The values of the optimal nominal interest rate are shown in the bottom left plot of Figure 3.1. Note that $(J)$ is the only case for which $i_{M}^{*}$ gets positive without violating Condition $(C 4)$, which happens for $b \geq 0.87$. We observe that in this region, the optimal nominal interest rate is below that prescribed by rule $(2-3)$, but for $b=0.90$ it gets above its steady-state level $(\bar{r})$. The bottom left plot of Figure 3.2 shows how the optimal medium-run nominal interest rate varies depending on crisis duration $(\mu)$ and the duration of the transitional state $(b)$. We see that the shorter the crisis is expected to be (lower $\mu$ ), sooner the optimal rate gets positive.

Figure 3.1 also presents in the bottom right plot a "zoomed" view of the bottom right corner of the top plot. This plot allows a better view of the differences in the cases with $i_{M}=0$ and $i_{M}=i_{M}^{*}$. It shows that in Case $(J)$, if the transitional state is expected to last for a longer period, implying $i_{M}^{*}>0$ $(b \geq 0.88)$, it generates slightly larger welfare losses. In fact, it would be better if agents expected it to last for around 7.7 periods $(b=0.87)$.

Figure 3.3 shows what happens to short- and medium-run output and inflation in this "zoomed" area for $b$. An important property of expecting a fully accommodative or an optimal monetary policy in the transitional state is that they create expectations of higher inflation and output in the medium run. But when the optimal policy is kept for a long time $(b \geq 0.88)$, making the optimal level of the nominal interest rate positive, medium-run output is expected to increase less, though inflation is still expected to be higher in the 
Figure 3.2: Optimal medium-run nominal interest rate $\left(i_{M}^{*}\right)$ as a function of $\mu$ and $b$
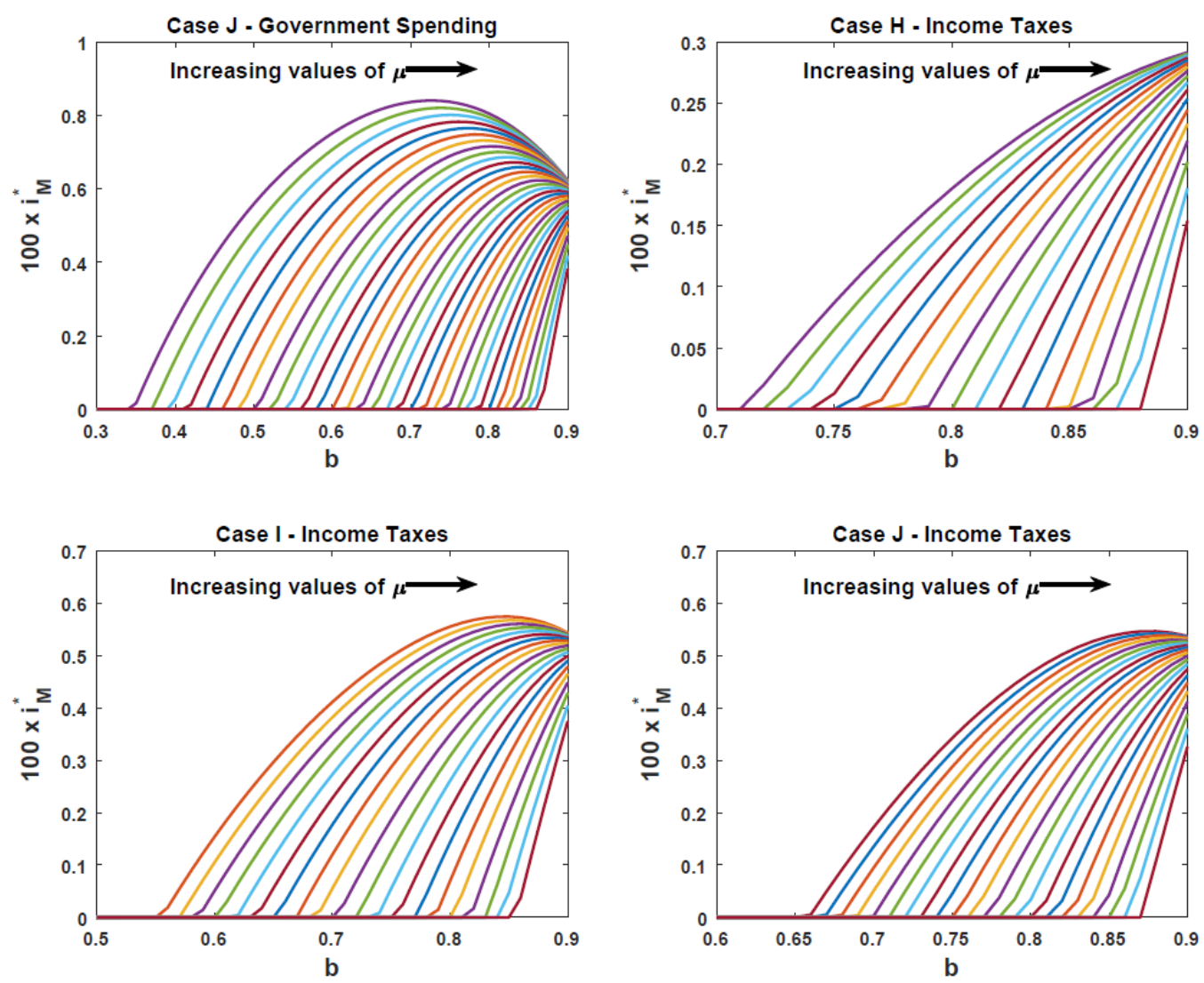

Note: This figure shows the values for the optimal medium-run nominal interest rate $\left(i_{M}^{*}\right)$ varying both the crisis duration $(\mu)$ and the duration of the transitional state $(b)$.

transitional state. This has a small impact on short run output and inflation. That is why there is a small increase in welfare losses.

It is interesting to note that, if the monetary authority is expected to adopt a fully accommodative policy in the transitional state, there are some values for $b \in[0.85,0.87]$ for which an increase in government spending during the crisis (Cases $(D)$ and $(H)$ ) imply in higher welfare losses than those observed with a delayed increase in this fiscal instrument (Cases $(G)$ and $(J)$ ). Figure 3.3 shows that his happens because the much larger increase in mediumrun output and inflation in Cases $(G)$ and $(J)$ compensate for the lack of fiscal stimulus in the short run, generating a smaller drop in short-run inflation, though short-run output still decreases less in Cases $(D)$ and $(H)$.

The policy combination that generates the least welfare losses are that in which the increase in government spending is implemented during the crisis and expected to continue after it is over, as long as it is fully accommodated by a zero medium-run nominal interest rate and that this transitional state is expected to last around 6.25 periods $(b=0.84)$. 
Figure 3.3: Short- and medium-run output and inflation with optimal transitional state monetary policy and government spending stimulus
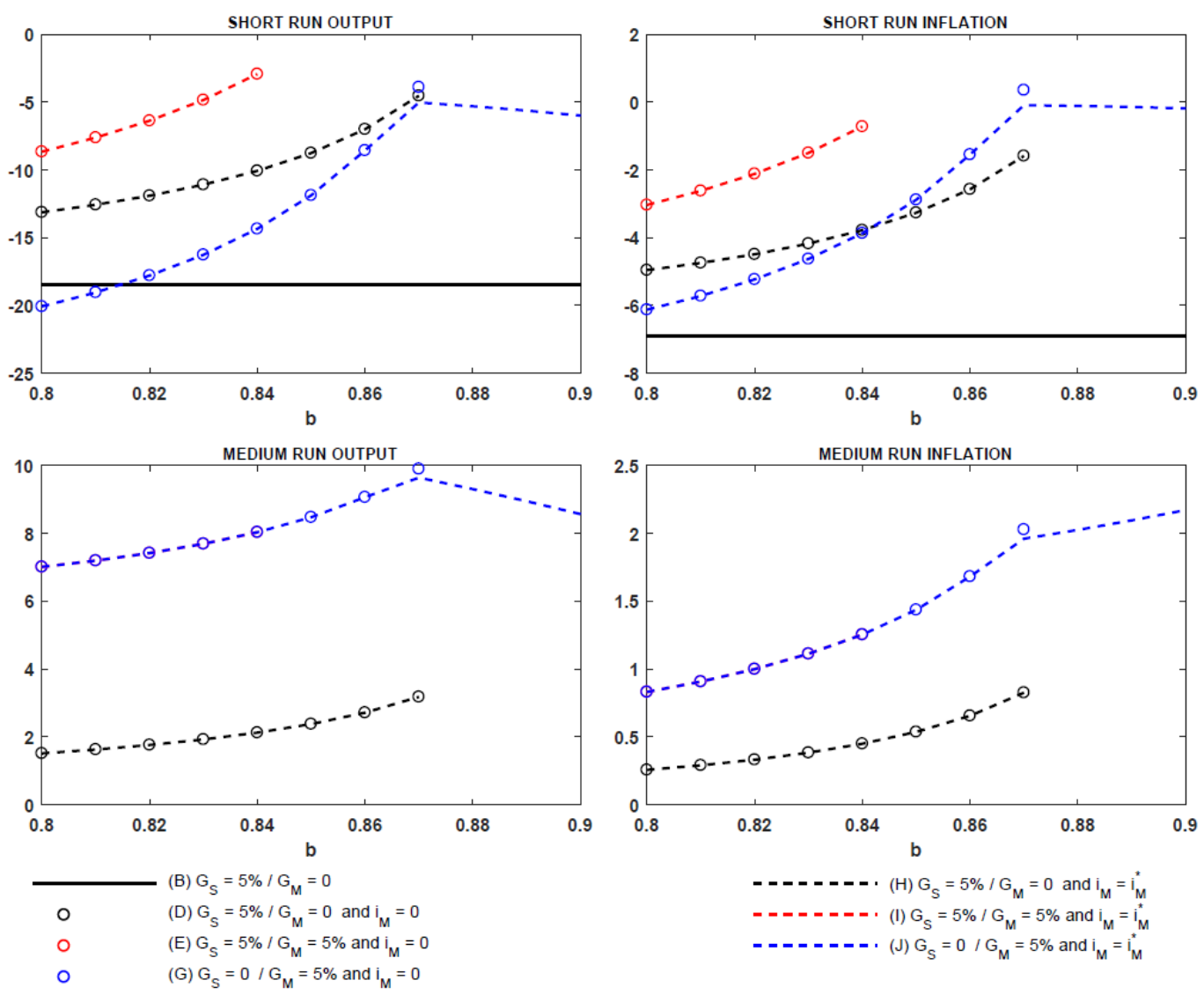

Note: This figure shows the behaviour of short- and medium-run output and inflation when fiscal policy is implemented through increases in government spending. It only displays the very end of the curves $(b \in[0.8,0.9])$ in order to compare the behaviour of these variables in those cases where the nominal interest rate is set at $i_{M}=0$ in the transitional state $((D),(E)$ and $(G))$ and those where it is optimally chosen $i_{M}=i_{M}^{*}((H),(I)$ and $(J))$.

Summarizing, this exercise showed that the losses in terms of welfare of a crisis in which the zero lower bound is binding can be very different. They depend not only on the policies adopted during the crisis, but also on those expected for the periods following the crisis. It is important that if the fiscal authority decides to keep stimulating the economy after the crisis is over, that this action is coordinated with an accommodative monetary policy. Besides, the longer it takes for the government spending stimulus to be implemented, larger will be the losses. In the extreme case where this stimulus is only implemented after the crisis is over, without monetary policy accommodation, it would be better not implementing any stimulus at all. On the other hand, the duration of the stimulus continuing after the crisis, associated with an optimal monetary policy, should not be expected to be too long because when the optimal nominal interest rate gets positive, welfare losses increases a little. 


\section{4 .2}

\section{Impact of Income Taxes Stimulus}

As shown in Chapter 2, an increase in income taxes can be stimulating during the crisis and it would continue to boost the economy as long as it keeps being fully accommodated by monetary policy. It also pointed out that a delay in the implementation of an income tax stimulus is not as harmful as a delay in increasing government spending, even without monetary policy accommodation. I bring these results to discuss what they imply in terms of welfare losses and what would be the optimal level of the nominal interest rate in the transitional state with this fiscal policy instrument.

Table 3.2 shows that, although reducing the drops in short-run output and inflation, if implemented only during the crisis (Case $(B)$ ), an increase in income taxes does not have an effect as large as that observed with an increase in government spending. Nevertheless, it is also able to reduce welfare losses in the economy (From 9.9 to 5.7). But in contrast to what happens when government spending is the instrument used by the fiscal authority, keeping the higher level of income taxes after the crisis is over, even without monetary policy accommodation (Case $(C)$ ), is able to reduce these losses even more (to 3.8).

When the stimulus is provided through increases in income taxes, for most values of the probability associated with the transitional state $(b)$, the solution for the optimal medium-run nominal interest rate is given by $i_{M}^{*}=0$. This makes Cases $(D),(E)$ and $(G)$ coincide with Cases $(H),(I)$ and $(J)$, respectively. However, as shown in the bottom left plot of Figure 3.4, in Cases $(H),(I)$ and $(J), i_{M}^{*}$ gets positive when $b$ is larger than $0.88,0.86$ and 0.87 , respectively. It even gets above the steady-state level for the nominal interest rate $(\bar{r})$ for $b=0.90$ in Cases $(I)$ and $(J)$, but it is always below the level implied by the rule (2-3). Figure 3.2 shows how the optimal level of the medium-run nominal interest rate varies with the duration of the crisis $(\mu)$ and of the transitional state $(b)$. As observed in Case $(J)$ for government spending, in Cases $(H),(I)$ and $(J)$ for taxes, the optimal rate gets positive with at a lower value for $(b)$, the smaller is the expected duration of the crisis $(\mu)$.

While $b \leq 0.86$, the policy combination that implies the lowest welfare losses is to the fiscal authority to increase income taxes during the crisis, and keep it when the crisis is over, a long as the monetary authority keeps accommodating it fully (Cases $(E)$ and $(I))^{4}$. However, if the transitional state is expected to be longer $(b \geq 0.88)$, with $i_{M}^{*}>0$, it would be better to the fiscal

\footnotetext{
${ }^{4}$ To be precise, this is still true for $b=0.87$, but just in Case $(I)$, since in Case $(E)$ Condition $(C 4)$ is not satisfied for $b$ equal or above this value.
} 
Figure 3.4: Welfare losses and optimal transitional state monetary policy with income tax stimulus
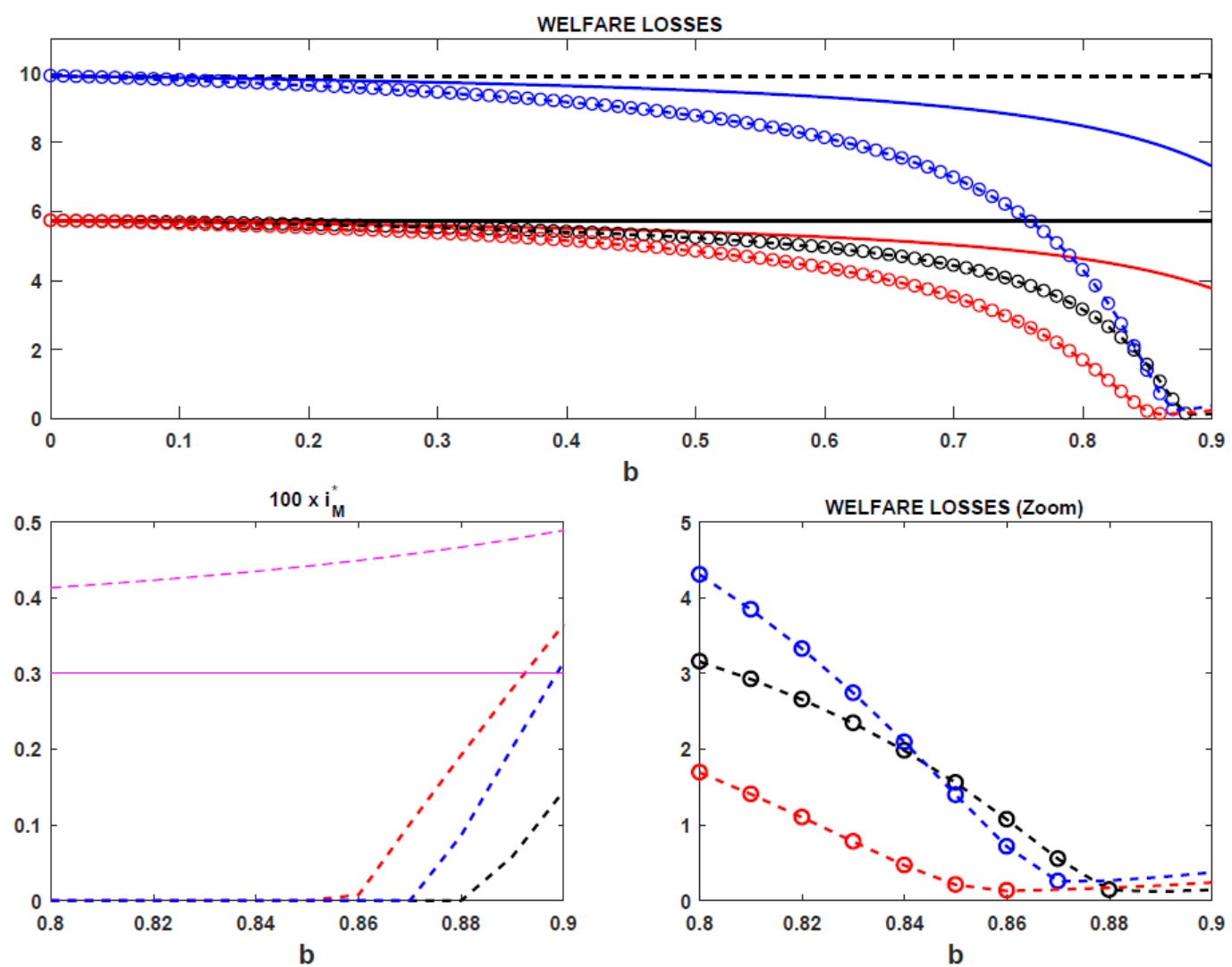

$$
\begin{aligned}
& - \text { (A) } \tau_{\mathrm{S}}^{1}=0 / \tau_{\mathrm{M}}^{1}=0 \\
& \text { (B) } \tau_{\mathrm{S}}^{1}=5 \% / \tau_{\mathrm{M}}^{1}=0 \\
& \text { (C) } \tau_{\mathrm{S}}^{1}=5 \% / \tau_{\mathrm{M}}^{1}=5 \% \text { and } \mathrm{i}_{\mathrm{M}}=\text { rule } \\
& \text { (D) } \tau_{\mathrm{S}}^{1}=5 \% / \tau_{\mathrm{M}}^{1}=0 \text { and } \mathrm{i}_{\mathrm{M}}=0
\end{aligned}
$$

- (E) $\tau_{\mathrm{S}}^{1}=5 \% / \tau_{\mathrm{M}}^{1}=5 \%$ and $\mathrm{i}_{\mathrm{M}}=0$

(F) $\tau_{\mathrm{S}}^{1}=0 / \tau_{\mathrm{M}}^{1}=5 \%$ and $\mathrm{i}_{\mathrm{M}}=$ rule

○

(G) $\tau_{\mathrm{S}}^{1}=0 / \tau_{\mathrm{M}}^{1}=5 \%$ and $\mathrm{i}_{\mathrm{M}}=0$

- - - - (I) $\tau_{\mathrm{S}}^{\mathrm{I}}=5 \% / \tau_{\mathrm{M}}^{\prime}=5 \%$ and $\mathrm{i}_{\mathrm{M}}=\mathrm{i}_{\mathrm{M}}^{*}$

- - - (J) $\tau_{\mathrm{S}}^{1}=0 / \tau_{\mathrm{M}}^{1}=5 \%$ and $\mathrm{i}_{\mathrm{M}}=\mathrm{i}_{\mathrm{M}}^{*}$

- - - $(\mathrm{H}) \tau_{\mathrm{S}}^{1}=5 \% / \tau_{\mathrm{M}}^{1}=0$ and $\mathrm{i}_{\mathrm{M}}=\mathrm{i}_{\mathrm{M}}^{*}$

Taylor rule nominal interest rate

Notes:

(i) The top plot displays welfare losses associated with each case as a function of probability $b$.

(ii) The bottom left plot presents the optimal level for the nominal interest rate in the transitional state $\left(i_{M}^{*}\right)$. It only plots the values for $b \in[0.80,0.90]$ because lowers values of $b$ imply $i_{M}^{*}=0$. Only very high values of $b$ imply $i_{M}^{*}>0$. This plot includes the steady-state nominal interest rate $(\bar{r})$ and its level implied by the rule (2-3) under Proposition 3.2.

footnotesize (iii) The bottom right graph shows a 'zoomed' view of the bottom right part of the top graph. It highlights the behaviour of welfare losses when $i_{M}^{*}>0$.

(iv) The plots that are discontinued before $b=0.90$ violate condition (C4) from Proposition 1 , thus the ZLB is no longer binding for these higher values of $b$. This happens for Cases $(D),(E)$ and $(G)$.

(v) In the bottom left plot, Case $(I)$ is 'hidden' since it implies $i_{M}^{*}=0$ for the values of $b$ for which the ZLB is still binding.

stimulus be reverted as soon as the crisis is over (Case $(H)$ ). As shown in the bottom left of Figure 3.4, the optimal nominal interest rate in the transitional state in Case $(H)$ is smaller than that obtained in Case $(I)$. Figure 3.5 shows that in the former, this implies a higher level of medium-run output and a lower level of medium-run inflation than in the latter. Nevertheless, in the short run, 
output and inflation are higher in Case $(I)$, making total welfare losses lower than in Case $(H)$.

Figure 3.5: Short- and medium-run output and inflation with optimal transitional state monetary policy and income taxes stimulus
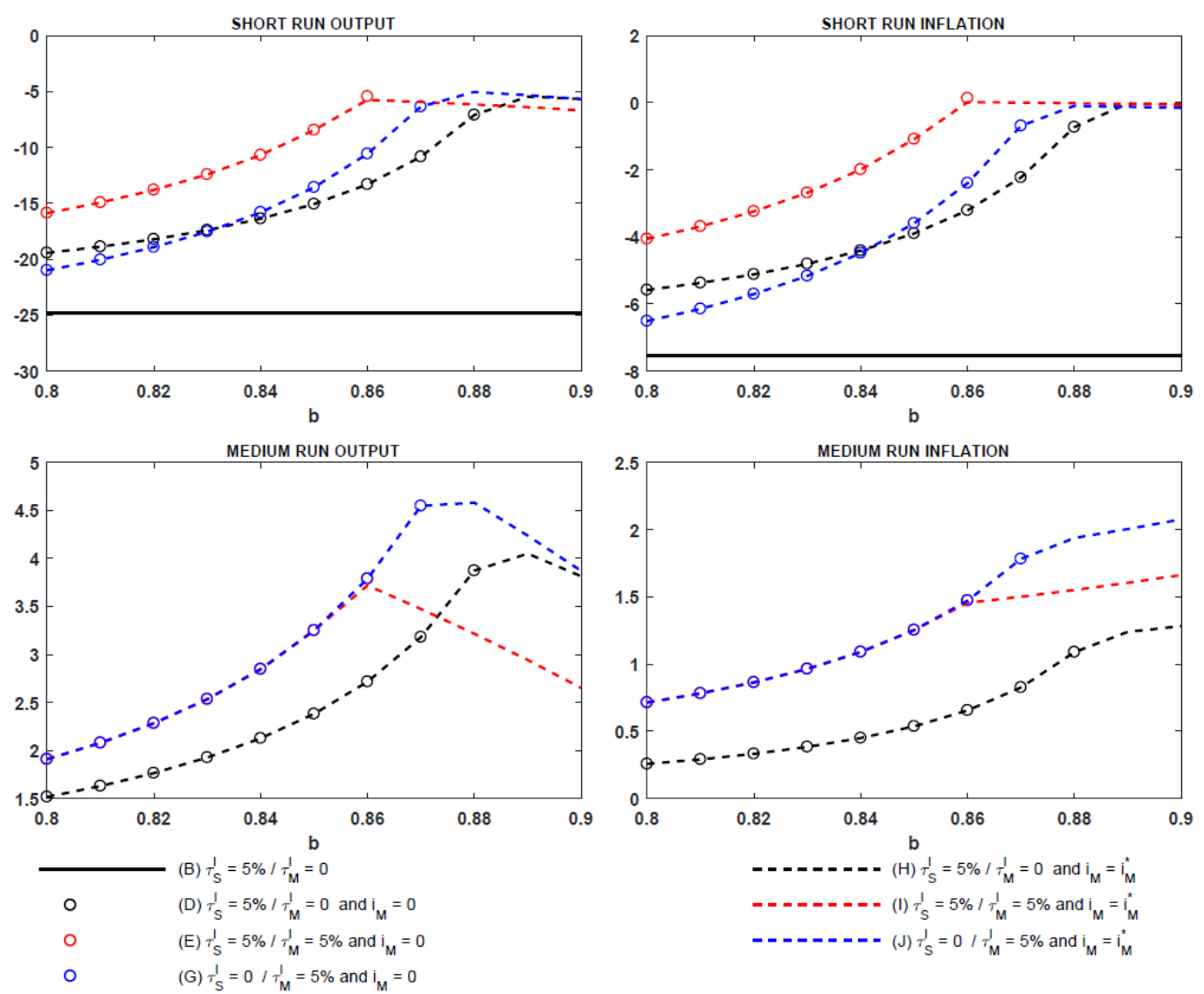

Note: This figure shows the behaviour of short- and medium-run output and inflation when fiscal policy is implemented through increases in income taxes. It only displays the very end of the curves $(b \in[0.8,0.9])$ in order to compare the behaviour of these variables in those cases where the nominal interest rate is set at $i_{M}=0$ in the transitional state $((D)$, $(E)$ and $(G))$ and those where it is optimally chosen $i_{M}=i_{M}^{*}((H),(I)$ and $(J))$.

A last issue to look at is the impact of delays in the implementation of a fiscal stimulus through an increase in income taxes (Cases $(F),(G)$ and $(J)$ ). It is natural to imagine that any modification in tax rates would take longer to be implemented than an increase in government spending. Figure 3.4 shows that in the case of taxes, even without monetary policy accommodation, it is still better to implement the stimulus after the crisis is over (Case $(F)$ ), than not implementing any stimulus at all (Case $(A))$.

Remember from Figure 2.8 in Chapter 2 that although decreasing output in the transitional state, this delay in the implementation of the fiscal stimulus is able to create expectations of higher inflation when the crisis is over. If this delayed implementation is accommodated by the monetary authority (Cases 
$(G)$ and $(J))$, losses are significantly reduced and can get even smaller than in the benchmark Case $(B)$ if this transitional state is expected to last for a longer period $(b \geq 0.86)$. They can even get close to those observed in Cases $(D)$ and $(H)$ or $(E)$ and $(I)$. In fact, for $b \in[0.85,0.87]$, Case $(G)$ and $(J)$ imply in smaller losses than in Cases $(D)$ and $(H)$. In this region, both $(H)$ and $(J)$ imply $i_{M}^{*}=0$. But the fiscal stimulus provided in Cases $(G)$ and $(J)$ increase more output and inflation in the transitional state than the sole action of monetary authority in Cases $(D)$ and $(H)$. This is able to compensate for the lack of fiscal stimulus in the crisis state, increasing short-run output and inflation, thus decreasing welfare losses.

It is interesting to note in Table 3.2 that, besides the benchmark Case $(B)$, in which the losses implied by an increase in government spending are smaller than those obtained with a stimulus through increases in income taxes, in the other cases, the stimulus through increases in income taxes gets losses that are smaller than or very similar to those with increases in government spending. ${ }^{5}$

\section{5 \\ Conclusion}

An economy facing a crisis situation caused by a shock that is large enough to make the nominal interest rate hit the zero lower bound imposes challenges to the conduction of monetary and fiscal policies. In this scenario, the monetary authority is limited in its actions, leaving the fiscal authority with an important role to implement policies to stimulate the economy. Nevertheless, this work showed that not only the policies implemented during the crisis are relevant to determine the depth of the recession and the welfare losses associated with it. Agents' expectations regarding policies that will be adopted when the crisis is over create a transitional state which is also crucial to attenuate or amplify the drops in output and inflation during the zero lower bound episode and the consequent welfare losses.

In this Chapter, I derived a welfare loss function in present discounted value terms in a three-state economy model that allowed to compare a set of policy combinations that could be implemented in this environment. This framework also supports the discussion of the choice of an optimal monetary policy in the transitional state, combined with the provision of a fiscal stimulus or not. Aiming at minimizing these losses, I showed that it is optimal for the monetary authority to signal that it will keep a fully accommodative

${ }^{5}$ Note that $b_{\max }$ is not equal under the same case with government spending stimulus or income taxes stimulus 
policy, with the nominal interest rate equal to zero, even when the zero lower bound is no longer binding. Losses will be further reduced if the action of the monetary authority is accompanied by the fiscal authority keeping the stimulus provided during the crisis for as long as the monetary authority keeps the nominal interest rate at zero. However, the continuation of the stimuli from both authorities should not be expected to be too long, since when the optimal nominal interest rate in the transitional state gets positive, welfare losses increase a little. It is also important for most part of the fiscal stimulus to be provided as soon as the crisis starts, although a delay when using income taxes is not as harmful as when government spending is the fiscal instrument being used.

This work showed that the discussion about fiscal policy multipliers at the zero lower bound should not be limited to the sole impact of a stimulus provided during the crisis. It should broaden its evaluation to account not only for what agents expect fiscal and monetary authorities would do when the crisis is over, but also how their actions are expected to be coordinated in future periods as well. It shows that it is important to communicate well not only the intentions of the monetary authority, but those from the fiscal authority as well.

A caveat that should be made in this analysis is that it is clearly impossible to expect that monetary and fiscal policy authorities would be able to perfectly fine tune their policies to be coordinated for exactly the time that would imply in smaller welfare losses. The exercise performed here looks at these small differences in the implications of different values for the probability $b$, and the timing of implementation of policies, only to discuss the impact of particular policy combinations, without claiming to be a type of policy prescription. 


\section{Bibliography}

[1] GALÍ, J.. Monetary Policy, Inflation, and the Business Cycle: An Introduction to the New Keynesian Framework and Its Applications. Economics Books. Princeton University Press, 2015.

[2] WOODFORD, M.. Interest and Prices: Foundations of a Theory of Monetary Policy. Princeton Univ. Press, 2003.

[3] SARGENT, T. J.; WALLACE, N.. Some unpleasant monetarist arithmetic. Quarterly Review, 1981.

[4] LOYO, E.. Tight Money Paradox on the Loose: A Fiscalist Hyperinflation. Manuscript, Kennedy School of Government, 1999.

[5] WOODFORD, M.. Fiscal requirements for price stability. Journal of Money, Credit and Banking, 33(3):669-728, 2001.

[6] COCHRANE, J. H.. Stepping on a rake: The fiscal theory of monetary policy. European Economic Review, 101:354-375, 2018.

[7] BLANCHARD, O.. Fiscal dominance and inflation targeting: Lessons from brazil. Working Paper 10389, National Bureau of Economic Research, 2004.

[8] BERRIEL, T. C.. Riscos Fiscais para a Política Monetária. Valor Econômico, Feb. 2014.

[9] DE BOLLE, M.. Brazil Needs to Abandon Inflation Targeting and Yield to Fiscal Dominance. Manuscript, Petterson Institute for International Economics, 2015.

[10] SCHYMURA, L. G.. Crise fiscal atrapalha bc, mas sistema de metas deve ser mantido. Carta de conjuntura, Instituto Brasileiro de Economia (IBRE-FGV), 2015.

[11] BERRIEL, T. C.. O peso do fator fiscal na dinâmica inflacionária. Valor Econômico, Nov. 2015.

[12] CARVALHO, C. V.. Possíveis razões para a inflação elevada. Valor Econômico, nov 2015. 
[13] INSPER. Há Dominância Fiscal no Brasil? - Evento do Centro de Finanças. Oct. 27 2015. Retrieved from https://youtu.be/FcnODT0jpkE.

[14] LEEPER, E. M.. Equilibria under active and passive monetary and fiscal policies. Journal of Monetary Economics, 27(1):129-147, 1991.

[15] LIU, Z.; WAGGONER, D. F. ; ZHA, T.. Asymmetric expectation effects of regime shifts in monetary policy. Review of Economic Dynamics, 12(2):284-303, 2009.

[16] CHO, S.; MORENO, A.. Global determinacy under monetary and fiscal policy switchings. Working Paper 2850500, SSRN, 2016.

[17] $\mathrm{CHO}, \mathrm{S}$.. Sufficient conditions for determinacy in a class of Markov-switching rational expectations models. Review of Economic Dynamics, 21:182-200, 2016.

[18] SIMS, C.. A simple model for study of the determination of the price level and the interaction of monetary and fiscal policy. Economic Theory, 4(3):381-99, 1994.

[19] DAVIG, T.; LEEPER, E. M.. Generalizing the Taylor Principle. American Economic Review, 97(3):607-635, 2007.

[20] LEEPER, E. M.; ZHA, T.. Modest policy interventions. Journal of Monetary Economics, 50(8):1673 - 1700, 2003.

[21] BHATTARAI, S.; LEE, J. W. ; PARK, W. Y.. Inflation dynamics: The role of public debt and policy regimes. Journal of Monetary Economics, 67:93-108, 2014.

[22] BIANCHI, F.; ILUT, C.. Monetary/Fiscal Policy Mix and Agents Beliefs. Review of Economic Dynamics, 26:113-139, 2017.

[23] BAELE, L.; BEKAERT, G.; CHO, S.; INGHELBRECHT, K. ; MORENO, A.. Macroeconomic Regimes. Journal of Monetary Economics, 70:51-71, 2015.

[24] FARMER, R. E. A.; WAGGONER, D. F. ; ZHA, T.. Understanding Markov-switching rational expectations models. Journal of Economic Theory, 144(5):1849-1867, 2009.

[25] FARMER, R. E. A.; WAGGONER, D. F. ; ZHA, T.. Minimal state variable solutions to Markov-switching rational expectations 
models. Journal of Economic Dynamics and Control, 35(12):2150-2166, 2011.

[26] CHO, S.; MORENO, A.. The forward method as a solution refinement in rational expectations models. Journal of Economic Dynamics and Control, 35(3):257-272, 2011.

[27] LJUNGQVIST, L.; SARGENT, T.. Recursive Macroeconomic Theory. MIT Press, 2004.

[28] CORSETTI, G.; MEIER, A. ; MULLER, G. J.. What Determines Government Spending Multipliers? Economic Policy, 27(72):521-565, 2012.

[29] COENEN, G.; ERCEG, C. J.; FREEDMAN, C.; FURCERI, D.; KUMHOF, M.; LALONDE, R.; LAXTON, D.; LINDÉ, J.; MOUROUGANE, A.; MUIR, D.; MURSULA, S.; DE RESENDE, C.; ROBERTS, J.; ROEGER, W.; SNUDDEN, S.; TRABANDT, M. ; IN'T VELD, J.. Effects of Fiscal Stimulus in Structural Models. American Economic Journal: Macroeconomics, 3(4):22-68, 2012.

[30] CHRISTIANO, L.; EICHENBAUM, M. ; REBELO, S.. When is the Government Spending Multiplier Large? Journal of Political Economy, 119(1):78-121, 2011.

[31] EGgerTSSON, G. B.. What Fiscal Policy is Effective at Zero Interest Rates? NBER chapters, National Bureau of Economic Research, 2011.

[32] DAVIG, T.; LEEPER, E. M.. Monetary-Fiscal Policy Interactions and Fiscal Stimulus. European Economic Review, 55(2):211-227, 2011.

[33] WOODFORD, M.. Simple Analytics of the Government Expenditure Multipliers. American Economic Journal: Macroeconomics, 3(1):135, 2011.

[34] EGGERTSSON, G. B.; WOODFORD, M.. Optimal Monetary and Fiscal Policy in a Liquidity Trap. NBER chapters, National Bureau of Economic Research, 2004.

[35] HALL, R. E.. What Accounts for the Changes in U.S. Fiscal Policy Transmission? Brooking Papers on Economic Activity, (2):183-249, 2009.

[36] SPILIMBERGO, A.; SCHINDLER, M. ; SYMANSKY, S.. Fiscal Multipliers. Staff Position Notes 11, International Monetary Fund, 2009. 
[37] RAMEY, V. A.; ZUBAIRY, S.. Government Spending Multipliers in Good Times and in Bad: Evidence from U.S. Historical Data. Working Paper 20719, National Bureau of Economic Research, 2014.

[38] ILZETZKI, E.; MENDOZA, E. G. ; VÉGH, C. A.. How big (small?) are fiscal multipliers? Journal of Monetary Economics, 60(2):239-254, Mar. 2013.

[39] EgGerTSSON, G. B.. The Paradox of Toil. Staff Reports 433, Federal Reserve Bank of New York, 2010.

[40] COGAN, J. F.; CWIK, T.; TAYLOR, J. B. ; WIELAND, V.. New Keynesian versus Old Keynesian Government Spending Multipliers. Journal of Economic Dynamics and Control, 34(3):281-295, 2010.

[41] CORSETTI, G.; KUESTER, K.; MEIER, A. ; MULLER, G. J.. Debt Consolidation and Fiscal Stabilization of Deep Recessions. American Economic Review, 100(2):41-45, 2010.

[42] DeneS, M.; EggertSSON, G. B. ; GILBUKH, S.. Deficits, Public Debt Dynamics and Tax and Spending Multipliers. The Economic Journal, 123(566):F133-F163, 2013.

[43] CLARIDA, R.; GALI, J. ; GERTLER, M.. The Science of Monetary Policy: A New Keynesian Perspective. Journal of Economic Literature, 37(4):1661-1707, dec 1999.

[44] EgGertSSON, G. B.. Fiscal Multipliers and Policy Coordination. Working Paper 628, Central Bank of Chile, 2011. 
A

\section{Appendix to Chapter 1}

\section{A.1}

\section{Economic Outlook and Expectations in 2015-2016}

Figure A.1: Fiscal Policy Deterioration

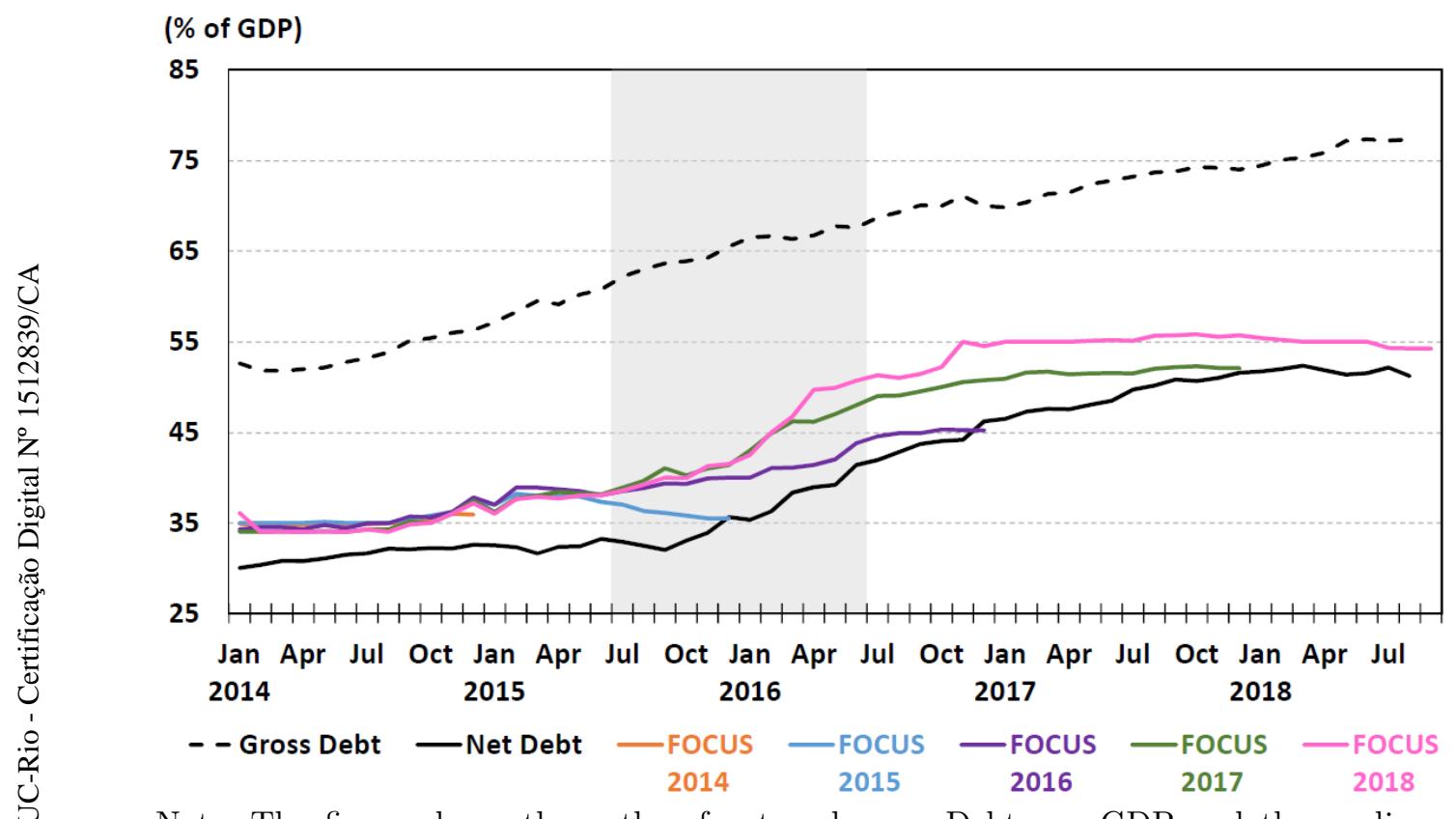

Note: The figure shows the paths of net and gross Debt-over-GDP and the median of professional forecasters' projections (FOCUS) for the net Debt-over-GDP. The shaded area represents the period in which the discussion about fiscal dominance was occurring in Brazil. We observe a deterioration of expectations during this period. 
Figure A.2: Inflation Expectations Desanchoring

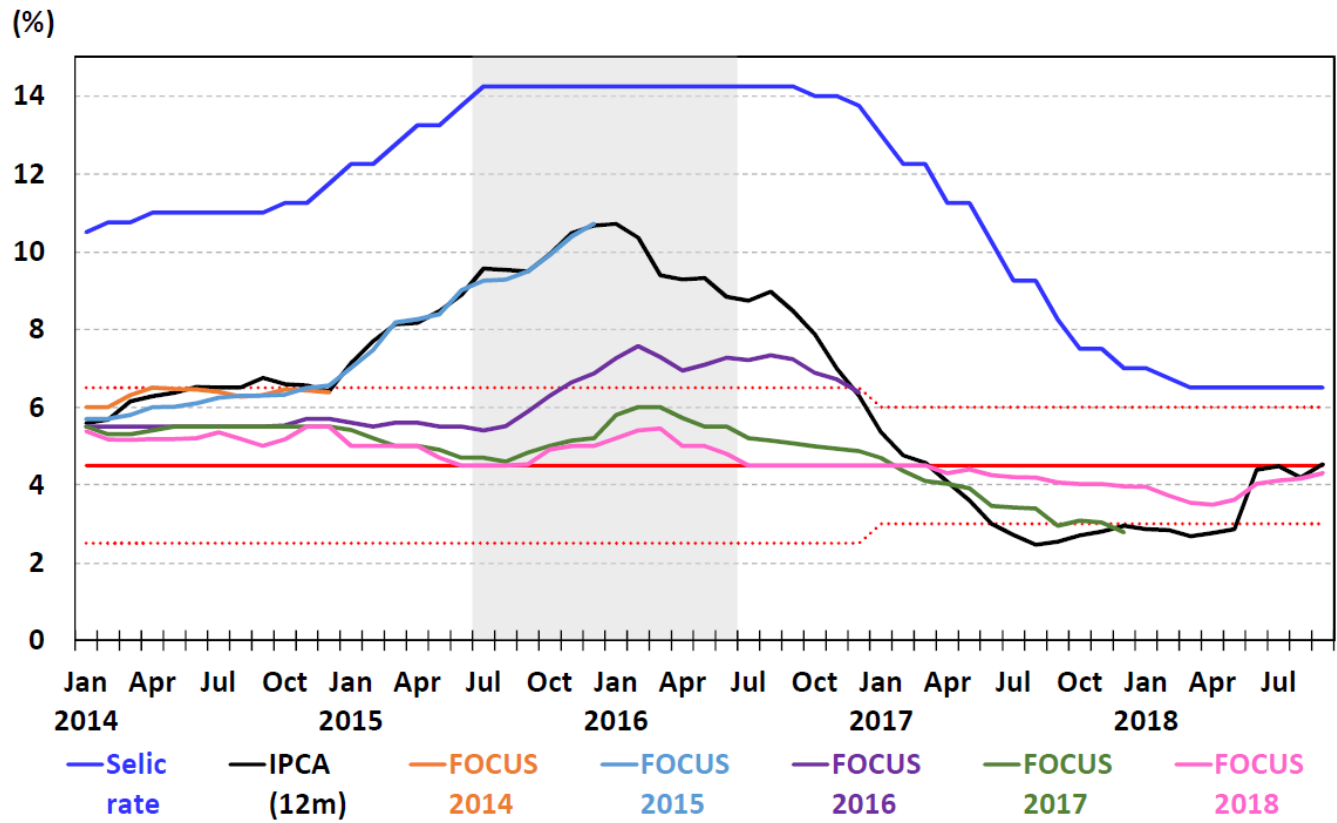

Note: The figure shows the path for 12-month inflation, the median of professional

forecasters' projections (FOCUS) for this variable and the behaviour of the monetary policy nominal interest rate (Selic). The red line represents the inflation target and the dot red line the target's tolerance band. The shaded area represents the period in which the discussion about fiscal dominance was occurring in Brazil. We observe that, although the nominal interest rate was kept in at a high level, inflation expectations for 2015 and 2016 were above the target upper band during this period.

Figure A.3: Recession Deepening

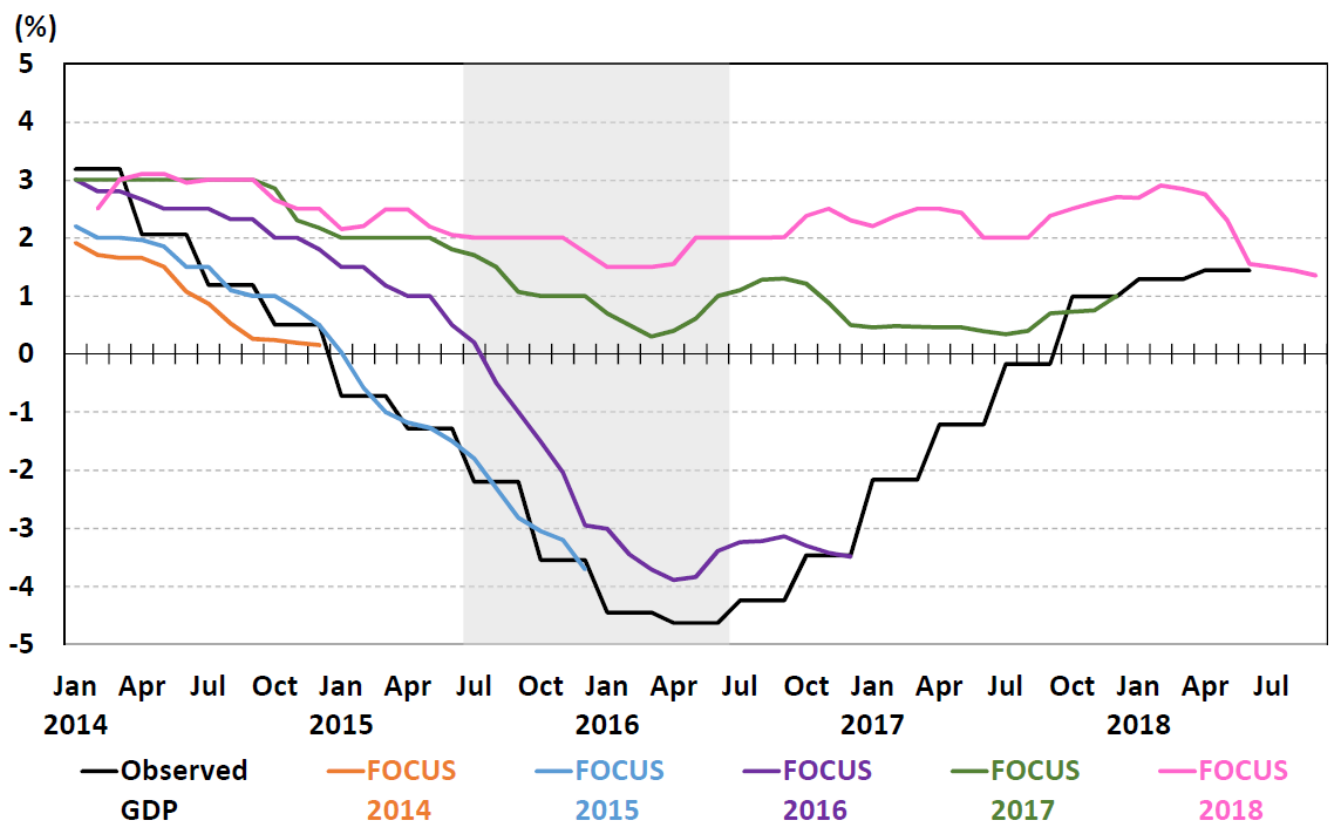

Note: The figure shows the path for 12-month GDP and the median of professional forecasters' projections (FOCUS) for this variable. The shaded area represents the period in which the discussion about fiscal dominance was occurring in Brazil. We observe that, during this period, recession was deepening and expectations were very negative as well. 


\section{A. 2}

IRFs varying $\left(p_{11}, p_{22}\right)$

Figure A.4: IRFs to a Monetary Policy Shock- AM/PF Regime
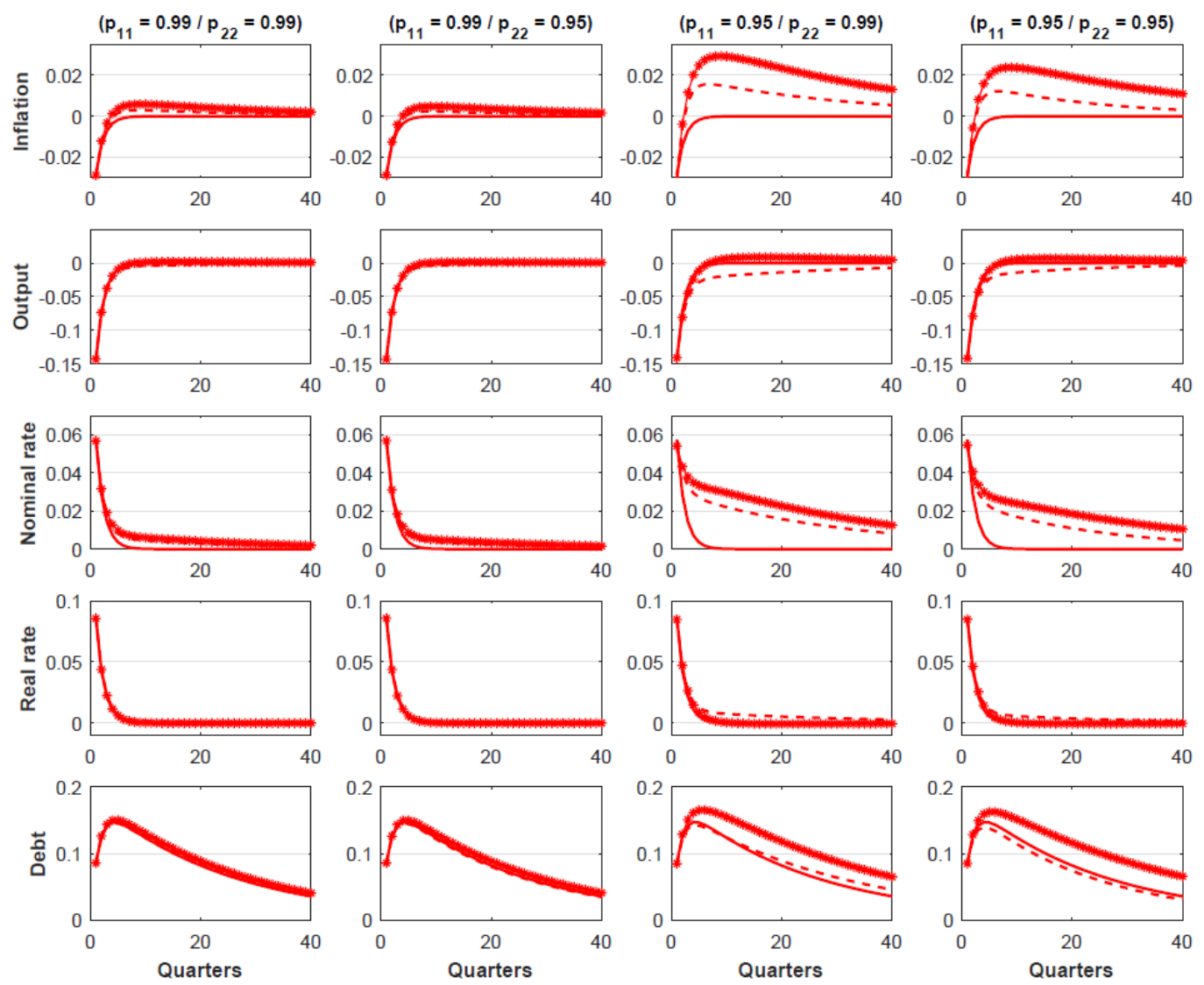

- - - - - - R1 MSRE - fixed

— - R1 MSRE - free

R1 LRE

Note: This figure presents the impulse responses if the economy is hit by a monetary policy shock while in the $\mathrm{AM} / \mathrm{PF}$ regime. It considers the four probabilities pairs and the policy parameters in the AM/PF regime $\left(\phi_{\pi, 1}=1.5\right.$ and $\left.\psi_{b, 1}=0.05\right)$ and in the $\mathrm{PM} / \mathrm{AF}$ regime $\left(\phi_{\pi, 2}=0.5\right.$ and $\left.\psi_{b, 2}=-0.03\right)$. 
Figure A.5: IRFs to a Monetary Policy Shock- PM/AF Regime
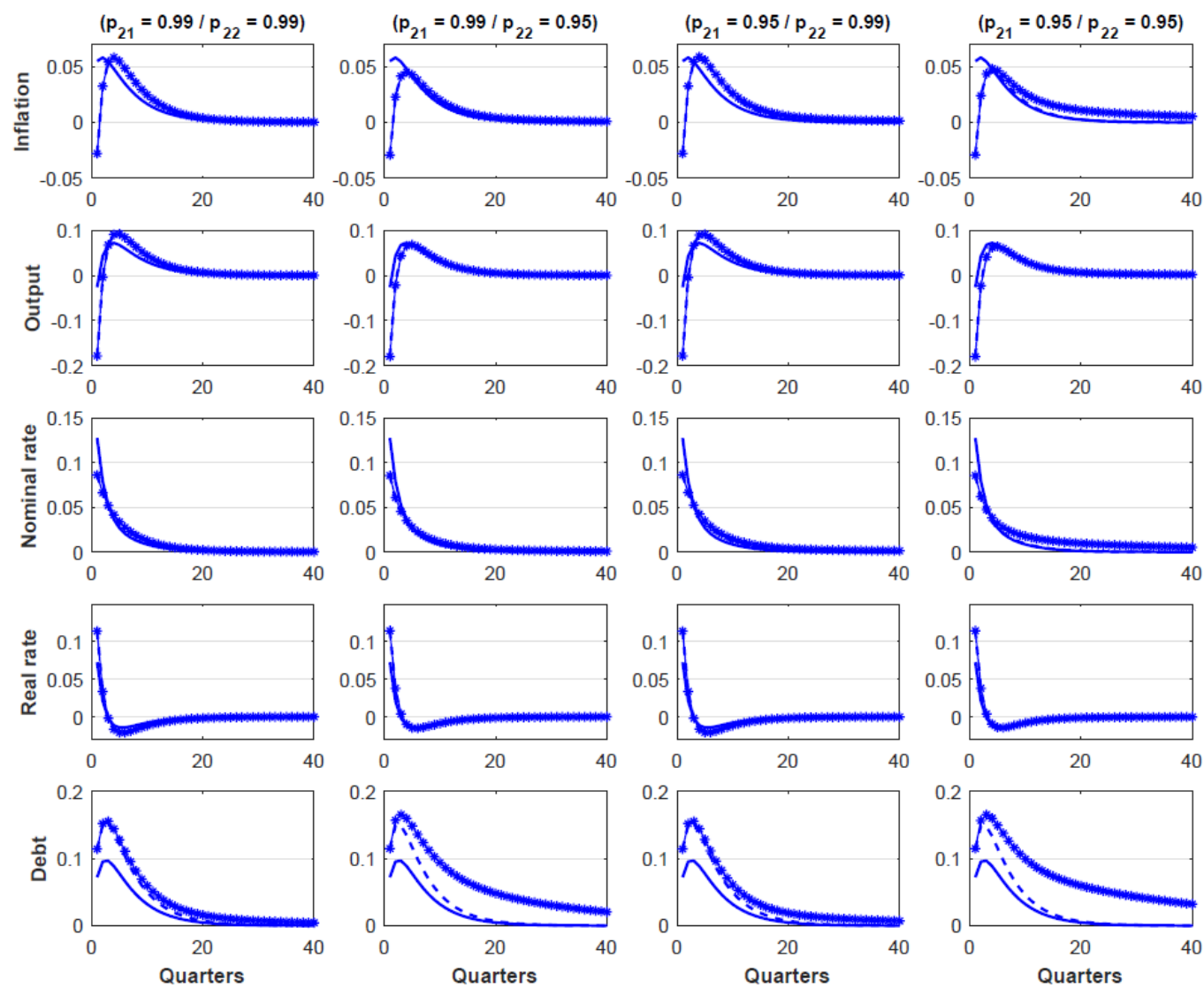

- - - - R2MSRE - fixed

$\longrightarrow$ - R2 MSRE - free

R2 LRE

Note: This figure presents the impulse responses if the economy is hit by a monetary policy shock while in the $\mathrm{PM} / \mathrm{AF}$ regime. It considers the four probabilities pairs and the policy parameters in the AM/PF regime $\left(\phi_{\pi, 1}=1.5\right.$ and $\left.\psi_{b, 1}=0.05\right)$ and in the $\mathrm{PM} / \mathrm{AF}$ regime $\left(\phi_{\pi, 2}=0.5\right.$ and $\left.\psi_{b, 2}=-0.03\right)$. 
Figure A.6: IRFs to a Fiscal Policy Shock- AM/PF Regime
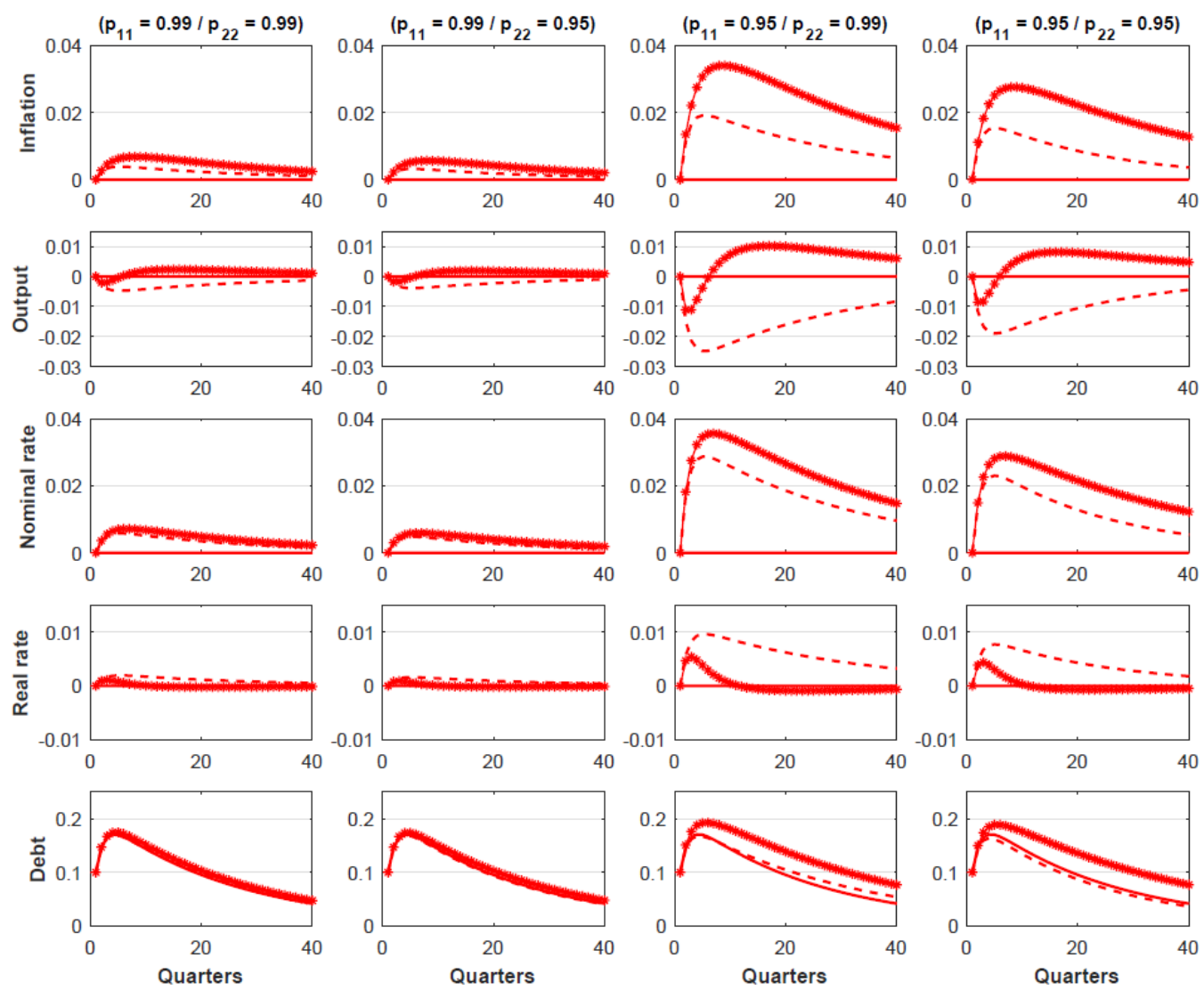

------ - R1 MSRE-fixed

— R1 MSRE - free

R1 LRE

Note: This figure presents the impulse responses if the economy is hit by a fiscal policy shock while in the $\mathrm{AM} / \mathrm{PF}$ regime. It considers the four probabilities pairs and the policy parameters in the $\mathrm{AM} / \mathrm{PF}$ regime $\left(\phi_{\pi, 1}=1.5\right.$ and $\left.\psi_{b, 1}=0.05\right)$ and in the $\mathrm{PM} / \mathrm{AF}$ regime $\left(\phi_{\pi, 2}=0.5\right.$ and $\left.\psi_{b, 2}=-0.03\right)$. 
Figure A.7: IRFs to a Fiscal Policy Shock- PM/AF Regime
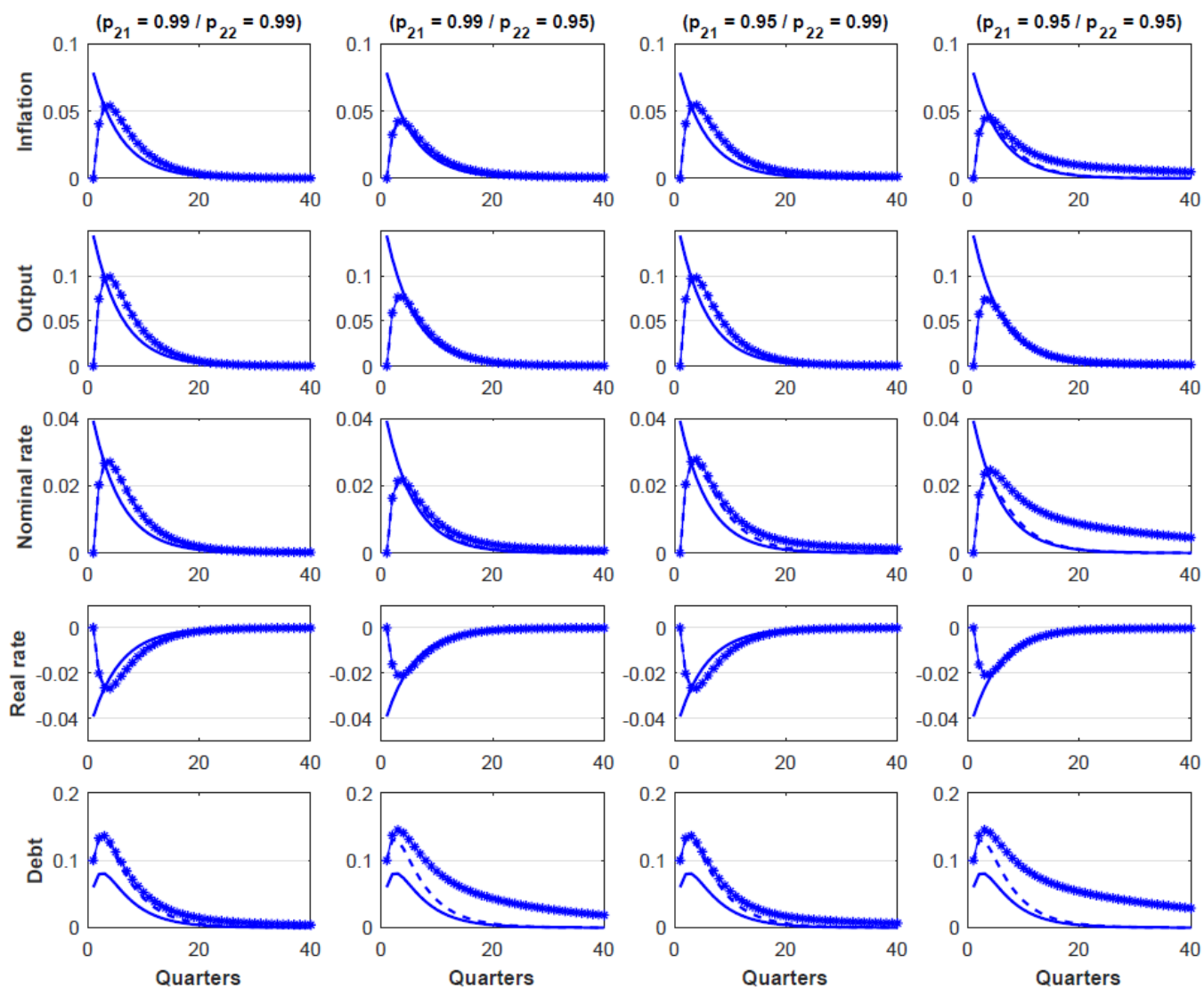

- - - - R2 MSRE - fixed

— - R2 MSRE - free

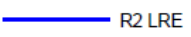

Note: This figure presents the impulse responses if the economy is hit by a fiscal policy shock while in the $\mathrm{PM} / \mathrm{AF}$ regime. It considers the four probabilities pairs and the policy parameters in the $\mathrm{AM} / \mathrm{PF}$ regime $\left(\phi_{\pi, 1}=1.5\right.$ and $\left.\psi_{b, 1}=0.05\right)$ and in the $\mathrm{PM} / \mathrm{AF}$ regime $\left(\phi_{\pi, 2}=0.5\right.$ and $\left.\psi_{b, 2}=-0.03\right)$. 
Figure A.8: IRFs to a Cost-Push Shock- AM/PF Regime
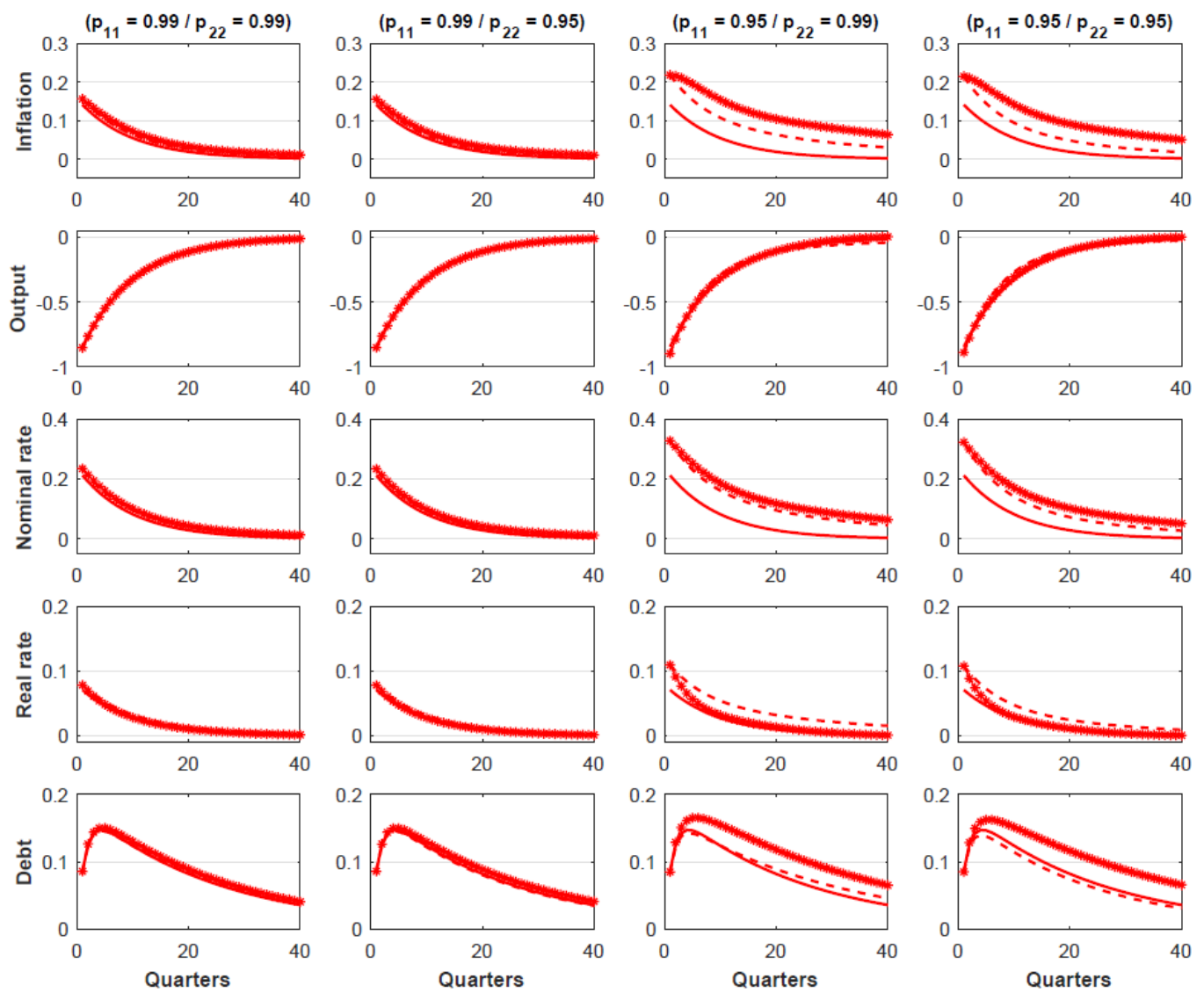

- - - - - - R1 MSRE-fixed

—

- R1 LRE

Note: This figure presents the impulse responses if the economy is hit by a cost-push shock while in the AM/PF regime. It considers the four probabilities pairs and the policy parameters in the AM/PF regime $\left(\phi_{\pi, 1}=1.5\right.$ and $\left.\psi_{b, 1}=0.05\right)$ and in the $\mathrm{PM} / \mathrm{AF}$ regime $\left(\phi_{\pi, 2}=0.5\right.$ and $\left.\psi_{b, 2}=-0.03\right)$. 
Figure A.9: IRFs to a Cost-Push Shock- PM/AF Regime
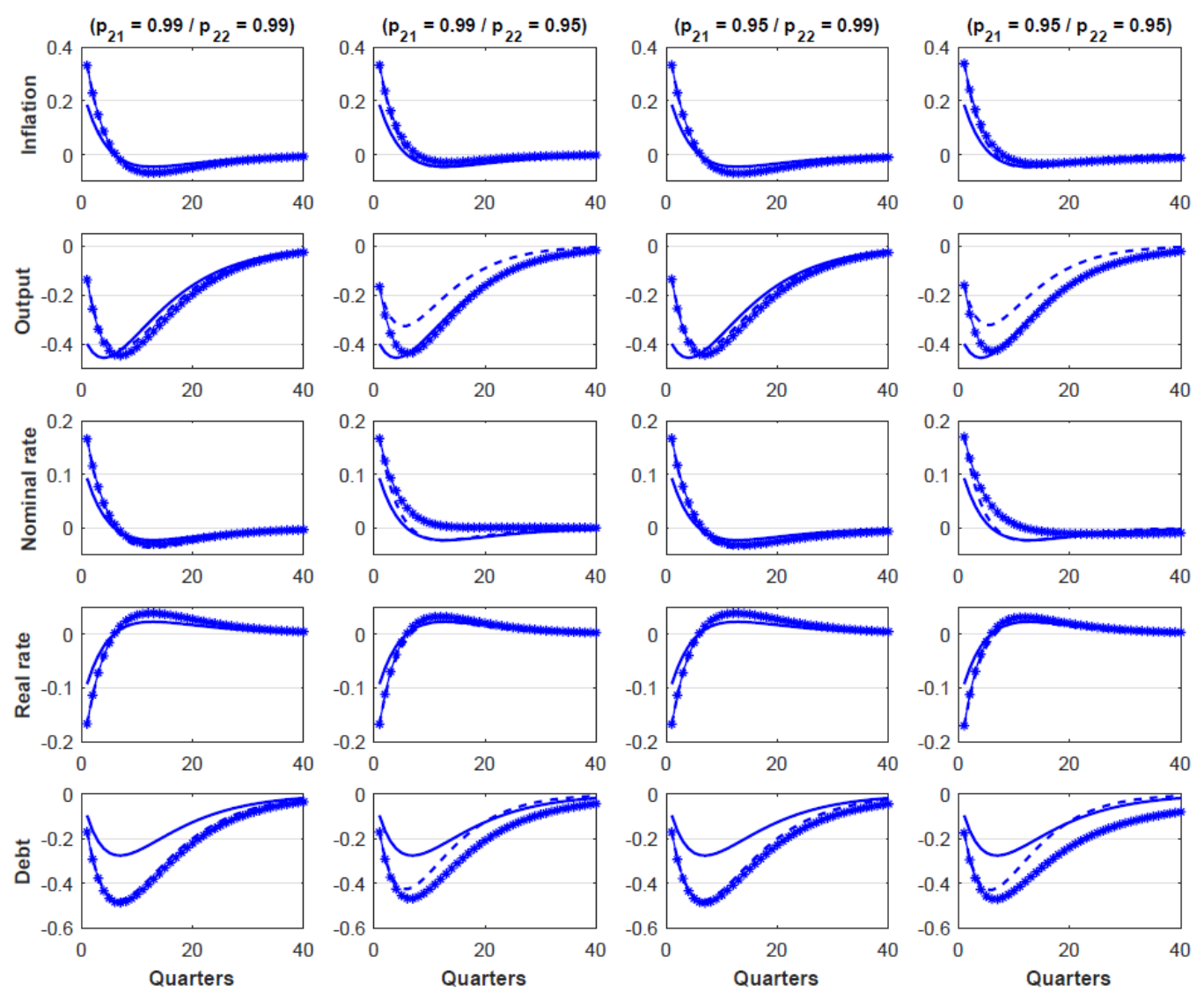

----- R2 MSRE - fixed

— R2 MSRE - free

R2 LRE

Note: This figure presents the impulse responses if the economy is hit by a cost-push shock while in the $\mathrm{PM} / \mathrm{AF}$ regime. It considers the four probabilities pairs and the policy parameters in the $\mathrm{AM} / \mathrm{PF}$ regime $\left(\phi_{\pi, 1}=1.5\right.$ and $\left.\psi_{b, 1}=0.05\right)$ and in the $\mathrm{PM} / \mathrm{AF}$ regime $\left(\phi_{\pi, 2}=0.5\right.$ and $\left.\psi_{b, 2}=-0.03\right)$. 
Figure A.10: IRFs to a Preference Shock- AM/PF Regime
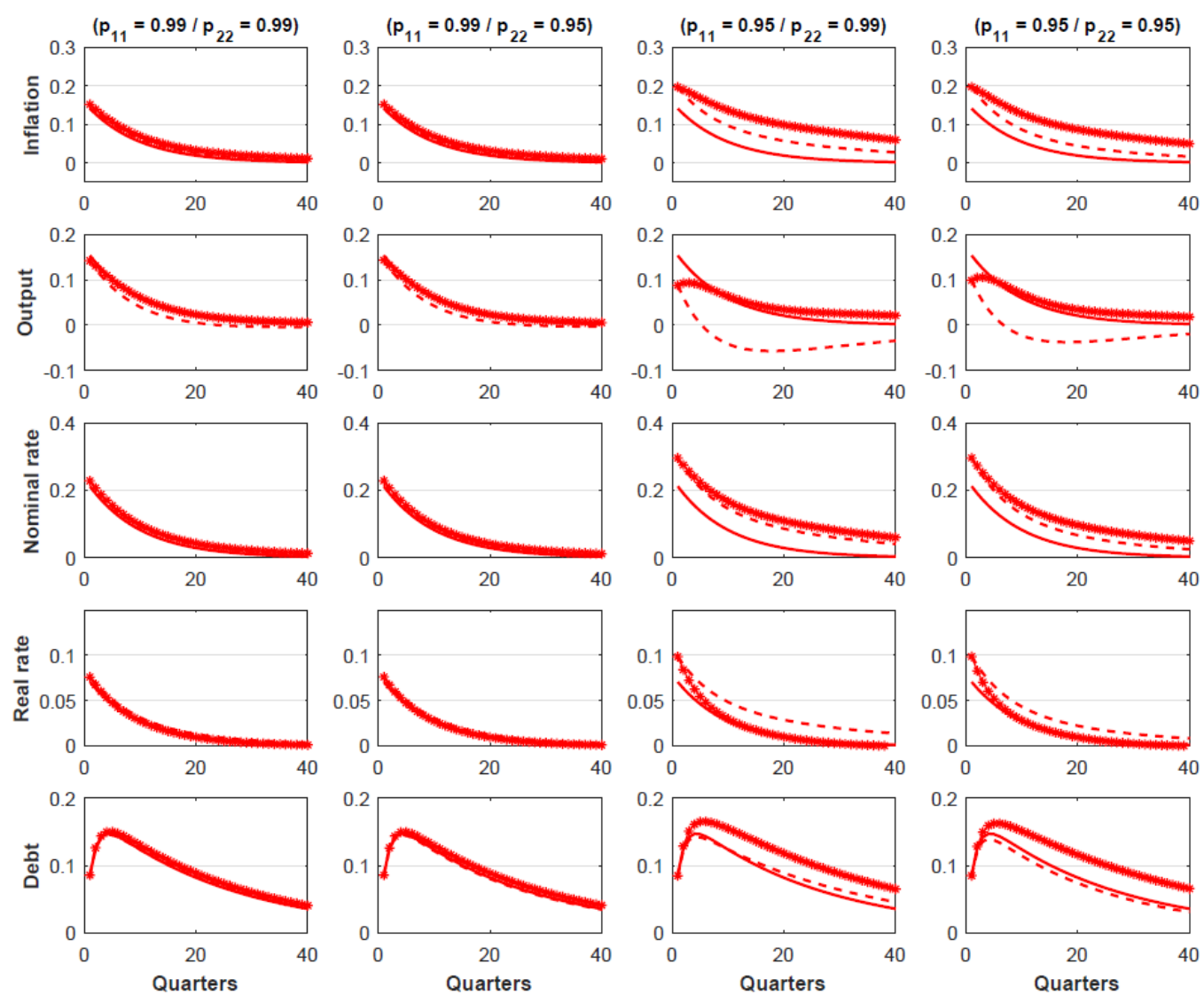

- - - - - - R1 MSRE - fixed

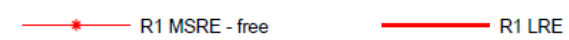

Note: This figure presents the impulse responses if the economy is hit by a preference shock while in the $\mathrm{AM} / \mathrm{PF}$ regime. It considers the four probabilities pairs and the policy parameters in the AM/PF regime $\left(\phi_{\pi, 1}=1.5\right.$ and $\left.\psi_{b, 1}=0.05\right)$ and in the $\mathrm{PM} / \mathrm{AF}$ regime $\left(\phi_{\pi, 2}=0.5\right.$ and $\left.\psi_{b, 2}=-0.03\right)$. 
Figure A.11: IRFs to a Preference Shock- PM/AF Regime
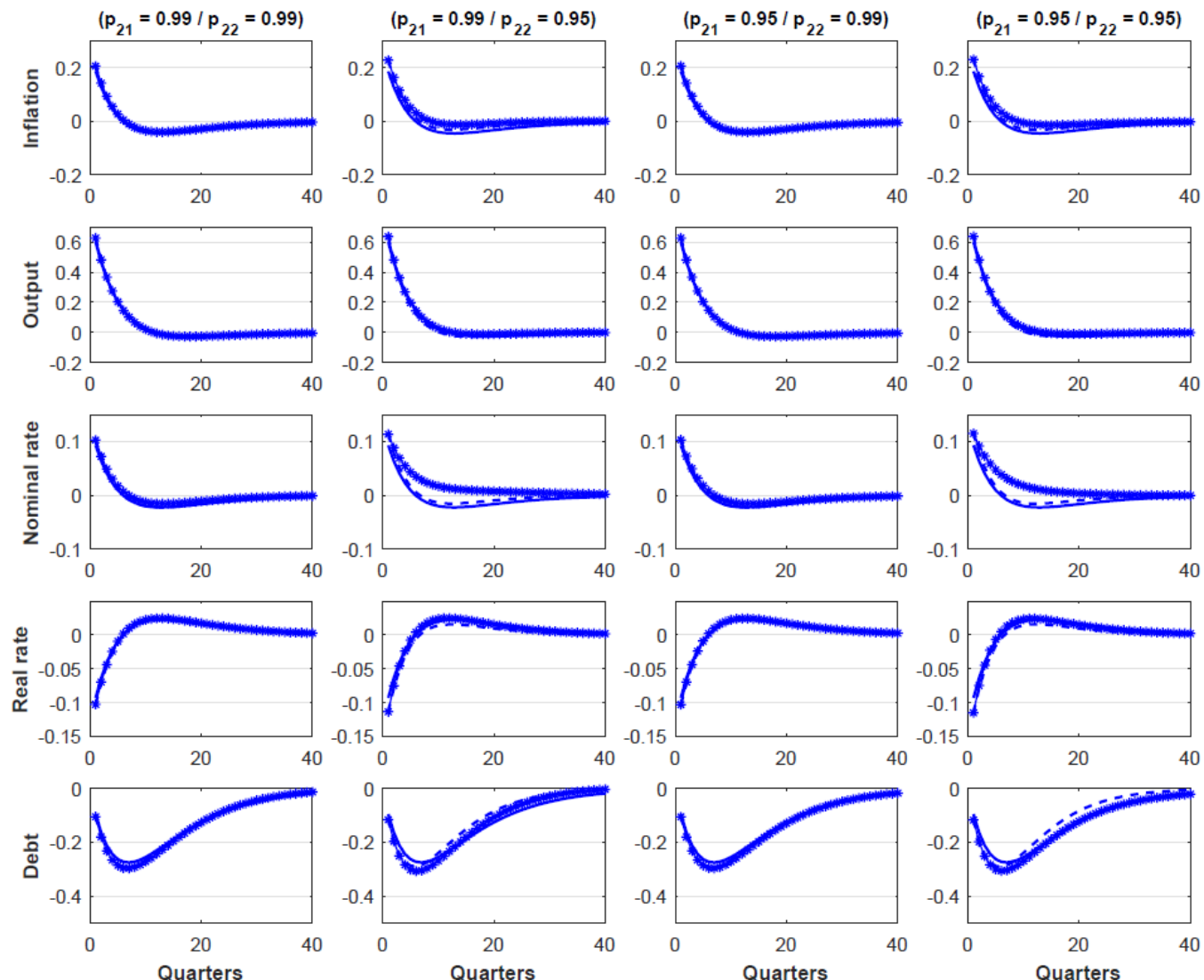

- - - - - R2 MSRE - fixed

— R2 MSRE - free

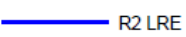

Note: This figure presents the impulse responses if the economy is hit by a preference shock while in the $\mathrm{PM} / \mathrm{AF}$ regime. It considers the four probabilities pairs and the policy parameters in the AM/PF regime $\left(\phi_{\pi, 1}=1.5\right.$ and $\left.\psi_{b, 1}=0.05\right)$ and in the $\mathrm{PM} / \mathrm{AF}$ regime $\left(\phi_{\pi, 2}=0.5\right.$ and $\left.\psi_{b, 2}=-0.03\right)$. 


\section{A.3}

\section{The Forward Method for Solving MSRE models}

This appendix shows how to obtain the forward solution and how to check for determinacy in the MSRE model under this type of solution. This result was established by Cho (17).

Proposition A.1 Consider model (1-9)-(1-10). For a given set of states $x_{t}$, $x_{t-1}$ and $z_{t}$ and the initial regime $s_{t}$ at time $t$, there exists a unique sequence of real-valued matrices $\left(\Omega_{h}\left(s_{t}\right), \Gamma_{h}\left(s_{t}\right), F_{h}\left(s_{t}, s_{t+1}\right)\right)$ for $h=1,2,3, \ldots$ such that

$$
x_{t}=E_{t}\left[M_{h}\left(s_{t}, s_{t+1}, \ldots, s_{t+h}\right) x_{t+h}\right]+\Omega_{h}\left(s_{t}\right) x_{t-1}+\Gamma_{h}\left(s_{t}\right) z_{t},
$$

where $\Omega_{1}\left(s_{t}\right)=B\left(s_{t}\right), \Gamma_{1}\left(s_{t}\right)=C\left(s_{t}\right), F_{1}\left(s_{t}, s_{t+1}\right)=M_{1}\left(s_{t}, s_{t+1}\right)=$ $A\left(s_{t}, s_{t+1}\right)$

$$
\begin{aligned}
\Omega_{h}\left(s_{t}\right)= & \left\{I_{n}-E_{t}\left[A\left(s_{t}, s_{t+1}\right) \Omega_{h-1}\left(s_{t+1}\right)\right]\right\}^{-1} B\left(s_{t}\right), \\
\Gamma_{h}\left(s_{t}\right)= & \left\{I_{n}-E_{t}\left[A\left(s_{t}, s_{t+1}\right) \Omega_{h-1}\left(s_{t+1}\right)\right]\right\}^{-1} C\left(s_{t}\right)+ \\
& E_{t}\left[F_{h}\left(s_{t}, s_{t+1}\right) \Gamma_{h-1}\left(s_{t+1}\right) R\left(s_{t+1}\right)\right] \\
F_{h}\left(s_{t}, s_{t+1}\right)= & \left\{I_{n}-E_{t}\left[A\left(s_{t}, s_{t+1}\right) \Omega_{h-1}\left(s_{t+1}\right)\right]\right\}^{-1} A\left(s_{t}, s_{t+1}\right),
\end{aligned}
$$

and

$$
M_{h}\left(s_{t}, s_{t+1}, \ldots, s_{t+k}\right)=F_{h}\left(s_{t}, s_{t+1}\right) M_{h-1}\left(s_{t+1}, \ldots, s_{t+h}\right),
$$

for $k=2,3, \ldots$ if the following regularity condition is satisfied for all $h>1$ and for all $s_{t}, s_{t+1}=1,2, \ldots, S$ :

$$
\left|I_{n}-E_{t}\left[A\left(s_{t}, s_{t+1}\right) \Omega_{h-1}\left(s_{t}\right)\right]\right| \neq 0 .
$$

Proof. See Cho (17).

It is simple to compute these sequences since the initial values $\Omega_{1}\left(s_{t}\right), \Gamma_{1}\left(s_{t}\right)$ and $F_{1}\left(s_{t}, s_{t+1}\right)$ are given by the model and the sequences $\Omega_{h}\left(s_{t}\right), \Gamma_{h}\left(s_{t}\right)$ and $F_{h}\left(s_{t}, s_{t+1}\right)$ are computed recursively. For instance, $E_{t}\left[A\left(s_{t}, s_{t+1}\right) \Omega_{h-1}\left(s_{t+1}\right)\right]=E\left[A\left(s_{t}, s_{t+1}\right) \Omega_{h-1}\left(s_{t+1}\right) \mid s_{t}=i\right]=$ $\sum_{j=1}^{S} p_{i j} A(i, j) \Omega_{k-1}(j)$ for all $s_{t}$ and all $k \geq 2$, with $\Omega_{1}\left(s_{t}\right)=B\left(s_{t}\right)$ The convergence property of these sequences and the forward solution are defined as follows.

Definition A.2 The MSRE model (1-9)-(1-10) is said to satisfy the forward convergence condition (FCC) if the coefficients of the state variables, 
$\left(\Omega_{h}\left(s_{t}\right), \Gamma_{h}\left(s_{t}\right)\right)$, in the forward representation (A-1) converge for every regime $s_{t}$ as $h$ tends to infinity. Under this condition, the model implies that

$$
x_{t}=\lim _{h \rightarrow \infty} E_{t}\left[M_{h}\left(s_{t}, s_{t+1}, \ldots, s_{t+h}\right) x_{t+h}\right]+\Omega^{*}\left(s_{t}\right) x_{t-1}+\Gamma^{*}\left(s_{t}\right) z_{t},
$$

where $\Omega^{*}\left(s_{t}\right)=\lim _{h \rightarrow \infty} \Omega_{h}\left(s_{t}\right)$ and $\Gamma^{*}\left(s_{t}\right)=\lim _{h \rightarrow \infty} \Gamma_{h}\left(s_{t}\right)$, and the forward solution is defined as the function of the state variables in the absence of the expectational effect in the forward representation in the limit above:

$$
x_{t}=\Omega^{*}\left(s_{t}\right) x_{t-1}+\Gamma^{*}\left(s_{t}\right) z_{t} .
$$

Definition A.3 A rational expectations solution to MSRE model (1-9)-(1-10) is said to satisfy the no-buble condition $(\mathbf{N B C})$ if the expectational term in the forward representation of the model, (A-1) converges to zero for every $s_{t}$ when expectations are formed with that solution:

$$
\lim _{h \rightarrow \infty} E_{t}\left[M_{h}\left(s_{t}, s_{t+1}, \ldots, s_{t+h}\right) x_{t+h}\right]=0_{n \times 1} .
$$

Proposition A.4 The forward solution (A-6) to MSRE model (1-9)-(1-10) is the unique fundamental solution that satisfies the no-buble condition.

Proof. See Cho \& Moreno (16)

\section{A.3.1}

\section{Necessary and Sufficient Conditions for Determinacy of the Forward} Solution

Before introducing the determinacy results of the forward solution, it is necessary to state the definition for spectral radius of a matrix.

Definition A.5 The spectral radius of an $n \times n$ matrix $M$ is defined as $r_{\sigma}(M)=\max _{1 \leq i \leq n}\left(\left|\lambda_{i}\right|\right)$, where $\lambda_{1}, \ldots, \lambda_{n}$ are the eigenvalues of $M$.

The following proposition from Cho \& Moreno (16) presents necessary and sufficient conditions for determinacy, indeterminacy and the case of no stable solution in a class of MSRE models.

Proposition A.6 Suppose that the forward solution (A-6) to model (1-9)(1-10) exists and it has the smallest spectral radius of the matrix $\bar{\Psi}_{\Omega^{*} \otimes \Omega^{*}}$ defined as:

$$
\bar{\Psi}_{\Omega^{*} \otimes \Omega^{*}}=\left[\begin{array}{ccc}
p_{11} \Omega^{*}(1) \otimes \Omega^{*}(1) & \cdots & p_{S 1} \Omega^{*}(1) \otimes \Omega^{*}(1) \\
\cdots & \cdots & \cdots \\
p_{1 S} \Omega^{*}(S) \otimes \Omega^{*}(S) & \cdots & p_{S S} \Omega^{*}(S) \otimes \Omega^{*}(S)
\end{array}\right]
$$


among all $\bar{\Psi}_{\Omega \otimes \Omega}$ within all fundamental solutions $x_{t}=\Omega\left(s_{t}\right) x_{t-1}+\Gamma\left(s_{t}\right) z_{t}$. Then, conditions $(C 1)-(C 3)$ for determinacy, indeterminacy and the case of no stable solution, defined in the table below, are necessary and sufficient.

$\begin{array}{ll}(C 1) \text { Determinacy } & r_{\sigma}\left(\bar{\Psi}_{\Omega^{*} \otimes \Omega^{*}}\right)<1 \text { and } r_{\sigma}\left(\Psi_{F^{*} \otimes F^{*}}\right) \leq 1 \\ (C 2) \text { Indeterminacy } & r_{\sigma}\left(\bar{\Psi}_{\Omega^{*} \otimes \Omega^{*}}\right)<1 \text { and } r_{\sigma}\left(\Psi_{F^{*} \otimes F^{*}}\right)>1 \\ & \text { existence of } M S S w_{t} \\ (C 3) \text { No stable solution } & r_{\sigma}\left(\bar{\Psi}_{\Omega^{*} \otimes \Omega^{*}}\right) \geq 1\end{array}$

where $\Psi_{F^{*} \otimes F^{*}}$ is defined as

$$
\Psi_{F^{*} \otimes F^{*}}=\left[\begin{array}{ccc}
p_{11} F^{*}(1,1) \otimes F^{*}(1,1) & \cdots & p_{1 S} F^{*}(1, S) \otimes F^{*}(1, S) \\
\cdots & \cdots & \cdots \\
p_{S 1} F^{*}(S, 1) \otimes F^{*}(S, 1) & \cdots & p_{S S} F^{*}(S, S) \otimes F^{*}(S, S)
\end{array}\right] .
$$

Proof. See Cho \& Moreno (16)

The first part of condition $(C 1)\left(r_{\sigma}\left(\bar{\Psi}_{\Omega^{*} \otimes \Omega^{*}}\right)<1\right)$ implies the meansquare stability of the forward solution. Its second part $\left(r_{\sigma}\left(\Psi_{F^{*} \otimes F^{*}}\right) \leq 1\right)$ ensures there is no MSS solution - not only fundamental, but also nonfundamental - other than the forward solution. Then, if $(C 1)$ holds, the model (1-9)-(1-10) is determinate in the MSS sense and the determinate equilibrium is given by the forward solution (A-6).

Proposition A.6 provides very tractable conditions for determinacy in the MSS sense to general MSRE models with predetermined variables.

Cho \& Moreno (16) highlight that while there is no known proof for establishing $r_{\sigma}\left(\bar{\Psi}_{\Omega^{*} \otimes \Omega^{*}}\right)$ is the smallest for general MSRE models, it is possible to examine this condition for the class of models that they analyse, which is the same used in this Chapter. They apply the method formulated by Farmer et. al. (25) which helps to find numerically all the fundamental solutions for the range of parameter spaces of interest. Thus, they apply this method to their models and find that $r_{\sigma}\left(\bar{\Psi}_{\Omega^{*} \otimes \Omega^{*}}\right) \leq r_{\sigma}\left(\bar{\Psi}_{\Omega \otimes \Omega}\right)$ for all their exercises. This guarantees that Proposition A.6 applies to the models analysed.

Thus, the procedure to solve this class of models can be summarized in the following steps:

1. Solve the model forward;

2. Compute the forward solution;

3. Check the maximum eigenvalues of matrices $\bar{\Psi}_{\Omega^{*} \otimes \Omega^{*}}$ and $\Psi_{F^{*} \otimes F^{*}}$ for determinacy. 


\section{A.4}

\section{Impulse Response Functions in the MSRE-FreeCase}

In the first case (MSRE-free), we assume that after the shock hits the economy, regime switches might happen along the impulse response path. In this case, we do not know the path until $s_{t+k-1}$, so it is necessary to account for all possible paths that can lead to $s_{t+k}$, starting from $s_{t}=i$.

We use the property of a Markov Chain ${ }^{1}$ with transition matrix $P$ for which the probability of moving from one value of the state to any other value in $k$ periods is given by $P\left(s_{t+k}=j \mid s_{t}=i\right)=P^{k}(i, j)$, where $P^{k}(i, j)$ represents the $(i, j)$-th element of $P^{k}$. Hence, using the forward solution (A-6), the expectation of the endogenous variables $(t+k)$-periods ahead is given by

$$
\begin{aligned}
E\left\{x_{t+k} \mid s_{t}=i\right\} & =E\left\{\left[\Omega^{*}\left(s_{t+k}=j\right) x_{t+k-1}+\Gamma^{*}\left(s_{t+k}=j\right) z_{t+k}\right] \mid s_{t}=i\right\} \\
& =\sum_{j=1}^{S} P^{k}(i, j)\left\{\left[\begin{array}{l}
\Omega^{*}\left(s_{t+k}=j\right) x_{t+k-1}+ \\
\Gamma^{*}\left(s_{t+k}=j\right) z_{t+k}
\end{array}\right] \mid s_{t}=i\right\} .
\end{aligned}
$$

Cho (17) shows how to compute these expectations recursively, using the forward solution and the exogenous process $z_{t}$ given by:

$$
\begin{aligned}
x_{t} & =\Omega^{*}\left(s_{t}\right) x_{t-1}+\Gamma^{*}\left(s_{t}\right) z_{t}, \\
z_{t} & =R\left(s_{t}\right) z_{t-1}+\epsilon_{t}, \quad \epsilon_{t} \sim\left(0_{m \times 1}, \Sigma\right) .
\end{aligned}
$$

The one-step ahead prediction of $x_{t+1}$, conditional on time $t$ information, including $s_{t}=i$, is given by:

$$
\begin{aligned}
E\left\{x_{t+1} \mid s_{t}=i\right\} & =E\left\{\left[\begin{array}{l}
\Omega^{*}\left(s_{t+1}=j\right) x_{t}+ \\
\Gamma^{*}\left(s_{t+1}=j\right)\left(R\left(s_{t+1}=j\right) z_{t}+\epsilon_{t+1}\right)
\end{array}\right] \mid s_{t}=i\right\} \\
& =E\left\{\Omega^{*}\left(s_{t+1}=j\right) \mid s_{t}=i\right\} x_{t}+ \\
& E\left\{\Gamma^{*}\left(s_{t+1}=j\right) R\left(s_{t+1}=j\right) \mid s_{t}=i\right\} z_{t} \\
& =F\left(s_{t}, 1\right) x_{t}+G\left(s_{t}, 1\right) z_{t}
\end{aligned}
$$

where $F\left(s_{t}, 1\right)=E\left\{\Omega^{*}\left(s_{t+1}=j\right) \mid s_{t}=i\right\} \quad$ and $\quad G\left(s_{t}, 1\right)=$ $E\left\{\Gamma^{*}\left(s_{t+1}=j\right) R\left(s_{t+1}=j\right) \mid s_{t}=i\right\}$. The $k$-step ahead prediction of $x_{t}$ is

${ }^{1}$ See Sargent \& Ljungqvist (27). 
then, given by:

$$
\begin{aligned}
& E\left\{x_{t+k} \mid s_{t}=i\right\}=E\left\{\left[\begin{array}{l}
\Omega^{*}\left(s_{t+k}=j\right) x_{t+k-1}+ \\
\Gamma^{*}\left(s_{t+k}=j\right)\left(R\left(s_{t+k}=j\right) z_{t+k-1}+\epsilon_{t+k}\right)
\end{array}\right] \mid s_{t}=i\right\} \\
& =E\left\{\Omega^{*}\left(s_{t+k}=j\right) x_{t+k-1} \mid s_{t}=i\right\}+ \\
& E_{t}\left\{\Gamma^{*}\left(s_{t+k}=j\right) R\left(s_{t+k}=j\right) z_{t+k-1} \mid s_{t}=i\right\} \\
& =E\left\{F\left(s_{t+1}, k-1\right) \Omega^{*}\left(s_{t+1}\right) \mid s_{t}=i\right\} x_{t}+ \\
& E\left\{\left[\begin{array}{l}
G\left(s_{t+1}, k-1\right)+ \\
F\left(s_{t+1}, k-1\right) \Gamma^{*}\left(s_{t+1}=j\right)
\end{array}\right] R\left(s_{t+1}=j\right) \mid s_{t}=i\right\} z_{t}, \\
& E\left\{x_{t+k} \mid s_{t}=i\right\}=F\left(s_{t}, k\right) x_{t}+G\left(s_{t}, k\right) z_{t},
\end{aligned}
$$

where $F\left(s_{t}, k\right)=E\left\{F\left(s_{t+1}, k-1\right) \Omega^{*}\left(s_{t+1}=j\right) \mid s_{t}=i\right\}$ and $G\left(s_{t}, k\right)=$ $E\left\{\left[\left(G\left(s_{t+1}, k-1\right)+F\left(s_{t+1}, k-1\right) \Gamma^{*}\left(s_{t+1}=j\right)\right) R\left(s_{t+1}=j\right)\right] \mid s_{t}=i\right\} \quad$ with $F\left(s_{t}, 0\right)=I_{n}$ and $G\left(s_{t}, 0\right)=0_{n \times m}$. Then, the impulse response function to the $l$-th innovation at time $t$, conditional on $s_{t}=i$, in the MSRE-free, is given by:

$$
\text { Free_IRF }\left(s_{t}, k\right)=\left(F\left(s_{t}, k\right) \Gamma^{*}\left(s_{t}\right)+G\left(s_{t}, k\right)\right) e_{l},
$$

for $k=0,1,2, \ldots$ where $e_{l}$ is an indicator vector of which the $l$-th element is 1 and 0 elsewhere.

\section{A.5 \\ Impulse Response Functions in the MSRE-Fixed Case}

In the second case (MSRE-fixed), we assume that the regime observed when the shocks hits the economy is kept along the impulse response path analyzed. Thus, we need to compute expectations of the endogenous variables $k$ periods ahead after the shock, assuming that if the state observed in period $t$ is $s_{t}=i$, then this state is observed in every period until $t+k\left(s_{t+k}=i, \forall k \geq 1\right)$. One should note that this is different from assuming that there is no regime switching. In the MSRE-fixed case, switches are allowed to occur, but they do not materializethemselves along the impulse response path.

We make use of the Markov Chain property that

$$
P\left(s_{t+k}=i \mid s_{t+k-1}=i, \forall k \geq 1\right)=p_{i i}^{k}
$$

where $p_{i i}$ represents the $(i, i)$-th element of $P$. Hence, using the forward solution (A-6) the expectation of the endogenous variables $t+k$-periods ahead is 
computed as

$E\left\{x_{t+k} \mid s_{t+k}=i, \forall k \geq 1\right\}=p_{i i}^{k}\left\{\left[\begin{array}{l}\Omega^{*}\left(s_{t+k}=i\right) x_{t+k-1}+ \\ \Gamma^{*}\left(s_{t+k}=i\right) z_{t+k}\end{array}\right] \mid s_{t+k}=i, \forall k \geq 1\right\}$.

The recursive formula for the impulse response functions in the MSRE-fixed case is obtained by analysing the pattern of the endogenous variables' expectations, after substituting the solutions forward for a few periods. Starting from period $t$, we have that when the shock hits the economy, we observe:

$$
\begin{aligned}
z_{t} & =\epsilon_{t}, \\
x_{t} & =\Gamma^{*}\left(s_{t}=i\right) z_{t}=\Gamma^{*}\left(s_{t}=i\right) \epsilon_{t} .
\end{aligned}
$$

We can use this to compute the endogenous variables expectations in period $t+1$. From the forward solution we have:

$$
\begin{aligned}
x_{t+1} & =\Omega^{*}\left(s_{t+1}=i\right) x_{t}+\Gamma^{*}\left(s_{t+1}=i\right) z_{t+1}, \\
z_{t+1} & =R\left(s_{t+1}=i\right) z_{t}+\epsilon_{t+1} .
\end{aligned}
$$

Substituting $x_{t}, z_{t}$ and $z_{t+1}$ into $x_{t+1}$ :

$x_{t+1}=\left[\Omega^{*}\left(s_{t+1}=i\right) \Gamma^{*}\left(s_{t}=i\right)+\Gamma^{*}\left(s_{t+1}=i\right) R\left(s_{t+1}=i\right)\right] \epsilon_{t}+\Gamma^{*}\left(s_{t+1}=i\right) \epsilon_{t+1}$.

Taking expectations conditional on $\left(s_{t+1}=s_{t}=i\right)$, we obtain:

$$
E\left\{x_{t+1} \mid s_{t+1}=s_{t}=i\right\}=p_{i i}\left\{\begin{array}{l}
\Omega^{*}\left(s_{t+1}=i\right) \Gamma^{*}\left(s_{t}=i\right)+ \\
\Gamma^{*}\left(s_{t+1}=i\right) R\left(s_{t+1}=i\right)
\end{array}\right\} \epsilon_{t} .
$$

Again, we can make use of this result to compute the expectations in period $t+2$ :

$$
\begin{aligned}
x_{t+2} & =\Omega^{*}\left(s_{t+2}=i\right) x_{t+1}+\Gamma^{*}\left(s_{t+2}=i\right) z_{t+2}, \\
z_{t+2} & =R\left(s_{t+2}=i\right) z_{t+1}+\epsilon_{t+2} .
\end{aligned}
$$

Substituting $x_{t}, x_{t+1}, z_{t}, z_{t+1}$ and $z_{t+2}$ into $x_{t+2}$ :

$$
\begin{gathered}
x_{t+2}=\Omega^{*}\left(s_{t+2}=i\right)\left[\left[\begin{array}{c}
\Omega^{*}\left(s_{t+1}=i\right) \Gamma^{*}\left(s_{t}=i\right)+ \\
\Gamma^{*}\left(s_{t+1}=i\right) R\left(s_{t+1}=i\right)
\end{array}\right] \epsilon_{t}+\Gamma^{*}\left(s_{t+1}=i\right) \epsilon_{t+1}\right]+ \\
\Gamma^{*}\left(s_{t+2}=i\right)\left[R\left(s_{t+2}=i\right) R\left(s_{t+1}=i\right) \epsilon_{t}+R\left(s_{t+2}=i\right) \epsilon_{t+1}+\epsilon_{t+2}\right]
\end{gathered}
$$




$$
\begin{gathered}
x_{t+2}=\left\{\begin{array}{l}
\Omega^{*}\left(s_{t+2}=i\right)\left[\Omega^{*}\left(s_{t+1=i}\right) \Gamma^{*}\left(s_{t}=i\right)+\Gamma^{*}\left(s_{t+1}=i\right) R\left(s_{t+1}=i\right)\right]+ \\
\Gamma^{*}\left(s_{t+2}=i\right) R\left(s_{t+2}=i\right) R\left(s_{t+1}=i\right)
\end{array}\right\} \epsilon_{t}+ \\
{\left[\Omega^{*}\left(s_{t+2}=i\right) \Gamma^{*}\left(s_{t+1}=i\right)+\Gamma^{*}\left(s_{t+2}=i\right) R\left(s_{t+2}=i\right)\right] \epsilon_{t+1}+\Gamma^{*}\left(s_{t+2}\right) \epsilon_{t+2} .}
\end{gathered}
$$

Taking expectations conditional on $\left(s_{t+2}=s_{t+1}=s_{t}=i\right)$, we get:

$$
\begin{gathered}
E\left\{x_{t+2} \mid s_{t+2}=s_{t+1}=s_{t}=i\right\}=E\left\{E\left\{E\left\{x_{t+2} \mid s_{t+2}=i\right\} \mid s_{t+1}=i\right\} \mid s_{t}=i\right\} \\
=p_{i i}^{2}\left\{\begin{array}{c}
\left.\Omega^{*}\left(s_{t+2}=i\right)\left[\begin{array}{c}
\Omega^{*}\left(s_{t+1}=i\right) \Gamma^{*}\left(s_{t}=i\right)+ \\
\Gamma^{*}\left(s_{t+1}=i\right) R\left(s_{t+1}=i\right)
\end{array}\right]+\right\} \epsilon_{t}, \\
\Gamma^{*}\left(s_{t+2}=i\right) R\left(s_{t+2}=i\right) R\left(s_{t+1}=i\right)
\end{array}\right\} \\
E\left\{x_{t+2} \mid s_{t+2}=s_{t+1}=s_{t}=i\right\}= \\
p_{i i} \Omega^{*}\left(s_{t+2}=i\right)\left\{p_{i i}\left[\begin{array}{l}
\Omega^{*}\left(s_{t+1}=i\right) \Gamma^{*}\left(s_{t}=i\right)+ \\
\Gamma^{*}\left(s_{t+1}=i\right) R\left(s_{t+1}=i\right)
\end{array}\right] \epsilon_{t}\right\}+ \\
p_{i i}^{2} \Gamma^{*}\left(s_{t+2}=i\right) R\left(s_{t+2}=i\right) R\left(s_{t+1}=i\right) \epsilon_{t}, \\
p_{i i} \Omega^{*}\left(s_{t+2}=i\right) E\left\{x_{t+1} \mid s_{t+1}=s_{t}=i\right\}+ \\
p_{i i}^{2} \Gamma^{*}\left(s_{t+2}=i\right) R\left(s_{t+2}=i\right) R\left(s_{t+1}=i\right) \epsilon_{t} .
\end{gathered}
$$

Moving one more period forward and computing the expectations in $t+3$ :

$$
\begin{aligned}
x_{t+3} & =\Omega^{*}\left(s_{t+3}=i\right) x_{t+2}+\Gamma^{*}\left(s_{t+3}=i\right) z_{t+3}, \\
z_{t+3} & =R\left(s_{t+3}=i\right) z_{t+2}+\epsilon_{t+3} .
\end{aligned}
$$

Substituting $x_{t}, x_{t+1}, x_{t+2}, z_{t}, z_{t+1}, z_{t+2}$ and $z_{t+3}$ into $x_{t+3}$ :

$$
\begin{aligned}
x_{t+3}= & \Omega^{*}\left(s_{t+3}=i\right) \times \\
& {\left[\begin{array}{l}
\left\{\Omega^{*}\left(s_{t+2}=i\right)\left[\begin{array}{l}
\Omega^{*}\left(s_{t+1}=i\right) \Gamma^{*}\left(s_{t}=i\right)+ \\
\Gamma^{*}\left(s_{t+1}=i\right) R\left(s_{t+1}=i\right) \\
\Gamma^{*}\left(s_{t+2}=i\right) R\left(s_{t+2}=i\right) R\left(s_{t+1}=i\right)
\end{array}\right]+\right\} \epsilon_{t}+ \\
{\left[\Omega^{*}\left(s_{t+2}=i\right) \Gamma^{*}\left(s_{t+1}=i\right)+\Gamma^{*}\left(s_{t+2}=i\right) R\left(s_{t+2}=i\right)\right] \epsilon_{t+1}+} \\
\Gamma^{*}\left(s_{t+2}=i\right) \epsilon_{t+2}
\end{array}\right]+} \\
& \Gamma^{*}\left(s_{t+3}=i\right) \times \\
& {\left[\begin{array}{l}
R\left(s_{t+3}=i\right) R\left(s_{t+2}=i\right) R\left(s_{t+1}=i\right) \epsilon_{t}+ \\
R\left(s_{t+3}=i\right) R\left(s_{t+2}=i\right) \epsilon_{t+1}+ \\
R\left(s_{t+3}=i\right) \epsilon_{t+2}+\epsilon_{t+3}
\end{array}\right] }
\end{aligned}
$$




$$
\begin{aligned}
& x_{t+3}=\left\{\begin{array}{l}
\Omega^{*}\left(s_{t+3}=i\right) \times \\
\left\{\begin{array}{l}
\Omega^{*}\left(s_{t+2}=i\right) \times \\
{\left[\begin{array}{l}
\Omega^{*}\left(s_{t+1}=i\right) \Gamma^{*}\left(s_{t}=i\right)+ \\
\Gamma^{*}\left(s_{t+1}\right) R\left(s_{t+1}=i\right)
\end{array}\right]+} \\
\Gamma^{*}\left(s_{t+2}=i\right) R\left(s_{t+2}=i\right) R\left(s_{t+1}=i\right)
\end{array}\right\}+ \\
\Gamma^{*}\left(s_{t+3}=i\right) R\left(s_{t+3}=i\right) R\left(s_{t+2}=i\right) R\left(s_{t+1}=i\right)
\end{array}\right\} \epsilon_{t}+ \\
&\left\{\begin{array}{l}
\Omega^{*}\left(s_{t+3}=i\right)\left[\begin{array}{l}
\Omega^{*}\left(s_{t+2}=i\right) \Gamma^{*}\left(s_{t+1}\right)+ \\
\Gamma^{*}\left(s_{t+2}=i\right) R\left(s_{t+2}=i\right)
\end{array}\right] \\
+\Gamma^{*}\left(s_{t+3}=i\right) R\left(s_{t+3}=i\right) R\left(s_{t+2}=i\right)
\end{array}\right\} \epsilon_{t+1}+ \\
& {\left[\begin{array}{l}
\Omega^{*}\left(s_{t+3}=i\right) \Gamma^{*}\left(s_{t+2}=i\right)+ \\
\Gamma^{*}\left(s_{t+3}=i\right) R\left(s_{t+3}=i\right)
\end{array}\right] \epsilon_{t+2}=i+\Gamma^{*}\left(s_{t+3}=i\right) \epsilon_{t+3} . }
\end{aligned}
$$

Taking expectations conditional on $\left(s_{t+3}=s_{t+2}=s_{t+1}=s_{t}=i\right)$ yields:

$$
\begin{gathered}
=p_{i i}^{3}\left\{\begin{array}{c}
E\left\{x_{t+3} \mid s_{t+3}=s_{t+2}=s_{t+1}=s_{t}=i\right\}= \\
\left.\Omega^{*}\left(s_{t+3}=i\right)\left\{\begin{array}{c}
E\left\{E\left\{E\left\{E\left\{x_{t+3} \mid s_{t+3}=i\right\} \mid s_{t+2}=i\right\} \mid s_{t+1}=i\right\} \mid s_{t}=i\right\} \\
\Omega^{*}\left(s_{t+2}=i\right)\left[\begin{array}{l}
\Omega^{*}\left(s_{t+1}=i\right) \Gamma^{*}\left(s_{t}=i\right)+ \\
\Gamma^{*}\left(s_{t+1}=i\right) R\left(s_{t+1}=i\right)
\end{array}\right]+ \\
\Gamma^{*}\left(s_{t+2}=i\right) R\left(s_{t+2}=i\right) R\left(s_{t+1}=i\right)
\end{array}\right\}+\right\} \epsilon_{t}, \\
\Gamma^{*}\left(s_{t+3}=i\right) R\left(s_{t+3}=i\right) R\left(s_{t+2}=i\right) R\left(s_{t+1}=i\right)
\end{array}\right\} \\
E\left\{x_{t+3} \mid s_{t+3}=s_{t+2}=s_{t+1}=s_{t}=i\right\}= \\
\left.p_{i i} \Omega^{*}\left(s_{t+3}=i\right) E\left\{x_{t+2} \mid s_{t+2}=s_{t+1}=s_{t}=i\right)\right\}+ \\
p_{i i}^{3} \Gamma^{*}\left(s_{t+3}=i\right) R\left(s_{t+3}=i\right) R\left(s_{t+2}=i\right) R\left(s_{t+1}=i\right) \epsilon_{t} .
\end{gathered}
$$

We can already see that the recursive forward substitution generates a pattern that allows us to conclude that the exogenous variables expectations in period $t+k$ are given by:

$$
\begin{gathered}
E\left\{x_{t+k} \mid s_{t+k}=s_{t+k-1}=\ldots=s_{t+1}=s_{t}=i\right\}= \\
p_{i i} \Omega^{*}\left(s_{t+k}=i\right) E\left\{x_{t+k-1} \mid s_{t+k-1}=s_{t+k-2}=\ldots=s_{t+1}=s_{t}=i\right\}+ \\
p_{i i}^{k} \Gamma^{*}\left(s_{t+k}=i\right)\left\{\prod_{q=1}^{k} R\left(s_{t+k}=i\right)\right\} \epsilon_{t} .
\end{gathered}
$$

Then, the impulse response function to the $l$-th innovation at time $t$, conditional on $s_{t}=i$, in the MSRE-fixed is given by:

$$
\text { Fixed_IRF }\left(s_{t}, 0\right)=\Gamma^{*}\left(s_{t}\right) \epsilon_{t} \text {, }
$$




$$
\begin{aligned}
\text { Fixed_IRF }\left(s_{t}, k\right)= & p_{i i} \Omega^{*}\left(s_{t+k}=i\right) \text { Fixed_IRF }\left(s_{t}, k-1\right)+ \\
& p_{i i}^{k} \Gamma^{*}\left(s_{t+k}=i\right)\left\{\prod_{q=1}^{k} R\left(s_{t+k}=i\right)\right\} \epsilon_{l},
\end{aligned}
$$

for $k=1,2, \ldots$ where $e_{l}$ is an indicator vector of which the $l$-th element is 1 and 0 elsewhere.

\section{A.6}

\section{Conditional and Unconditional Volatilities}

We use the results in Baele et. al. (23) to obtain the formulae used to compute the volatilities of the endogenous variables in Section 1.5. In their formulation, the shock processes are i.i.d., thus presenting no persistence. In our case, we need to consider the AR(1) process of the shocks. Hence, we can not directly apply the formulae presented in Baele et. al. (23) because we need to consider the persistence of the shocks. We start from the forward solution

$$
\begin{aligned}
& x_{t}=\Omega\left(s_{t}\right) x_{t-1}+\Gamma\left(s_{t}\right) z_{t}, \\
& z_{t}=R z_{t-1}+\epsilon_{t}, \quad \epsilon_{t} \sim \mathcal{N}(0, \Sigma)
\end{aligned}
$$

and rewrite it as

$$
\begin{aligned}
& x_{t}=\Omega\left(s_{t}\right) x_{t-1}+\Gamma\left(s_{t}\right) R z_{t-1}+\Gamma\left(s_{t}\right) \epsilon_{t}, \\
& x_{t}=\Omega\left(s_{t}\right) x_{t-1}+\Gamma\left(s_{t}\right) R z_{t-1}+V\left(s_{t}\right) \xi_{t}, \quad \xi_{t} \sim \mathcal{N}\left(0, I_{n}\right)
\end{aligned}
$$

where $V\left(s_{t}\right)=\Gamma\left(s_{t}\right) \Sigma^{1 / 2}$, with $\Sigma$ being the matrix of variance of the shocks. From the previous equation we have:

$$
x_{t} x_{t}^{\prime}=\Omega\left(s_{t}\right) x_{t-1} x_{t-1}^{\prime} \Omega^{\prime}\left(s_{t}\right)+\Gamma\left(s_{t}\right) R z_{t-1} z_{t-1}^{\prime} R^{\prime} \Gamma^{\prime}\left(s_{t}\right)+V\left(s_{t}\right) \xi_{t} \xi_{t}^{\prime} V^{\prime}\left(s_{t}\right)
$$

Taking the expectation of this term, conditional on being in state $s_{t}=i$ :

$$
\begin{aligned}
E\left[x_{t} x_{t}^{\prime} \mid s_{t}=i\right]= & \Omega\left(s_{t}=i\right) E\left[x_{t-1} x_{t-1}^{\prime} \mid s_{t}=i\right] \Omega^{\prime}\left(S_{t}=i\right)+ \\
& \Gamma\left(s_{t}=i\right) R E\left[z_{t-1} z_{t-1}^{\prime}\right] R^{\prime} \Gamma^{\prime}\left(s_{t}=i\right)+V\left(s_{t}=i\right) V^{\prime}\left(s_{t}=i\right) .
\end{aligned}
$$

Denote $P_{i}=\operatorname{Pr}\left(s_{t}=i\right)$ the long-run probability of being in state $\left(s_{t}=i\right)$ satisfying

$$
\sum_{i=1}^{S} P_{i j} P_{i}=P_{j} \text { and } \sum_{i=1}^{S} P_{i}=1,
$$


which we use to compute $E\left[x_{t-1} x_{t-1}^{\prime} \mid s_{t}=i\right]$ :

$$
\begin{gathered}
E\left[x_{t-1} x_{t-1}^{\prime} \mid s_{t}=i\right]=\sum_{j=1}^{S} E\left[x_{t-1} x_{t-1}^{\prime} \mid s_{t-1}=j\right] \operatorname{Pr}\left(s_{t-1}=j \mid s_{t}=i\right) \\
=\sum_{j=1}^{S} E\left[x_{t-1} x_{t-1}^{\prime} \mid s_{t-1}=j\right] \frac{\operatorname{Pr}\left(s_{t-1}=j\right)}{\operatorname{Pr}\left(s_{t}=i\right)} \operatorname{Pr}\left(s_{t}=i \mid s_{t-1}=j\right) \\
=\sum_{j=1}^{S} E\left[x_{t-1} x_{t-1}^{\prime} \mid s_{t-1}=j\right] \frac{P_{j}}{P_{i}} P_{j i} .
\end{gathered}
$$

Using that the variance of an $\operatorname{AR}(1)$ process $y_{t}=\rho y_{t-1}+u_{t}, u_{t} \sim$ $\mathcal{N}\left(0, \sigma_{u}^{2}\right)$, is given by $\frac{\sigma_{u}^{2}}{1-\rho_{u}^{2}}$, we have that using the values of $R$ and $\Sigma$ given in Section 1.3.1, we obtain:

$$
\Xi_{z}=R E\left[z_{t-1} z_{t-1}^{\prime}\right] R^{\prime}=\left[\begin{array}{cccc}
\rho_{A S}^{2} \frac{\sigma_{A S}^{2}}{1-\rho_{A S}^{2}} & 0 & 0 & 0 \\
0 & \rho_{I S}^{2} \frac{\sigma_{I S}^{2}}{1-\rho_{I S}^{2}} & 0 & 0 \\
0 & 0 & \rho_{M P}^{2} \frac{\sigma_{M P}^{2}}{1-\rho_{M P}^{2}} & 0 \\
0 & 0 & 0 & \rho_{F P}^{2} \frac{\sigma_{F P}^{2}}{1-\rho_{F P}^{2}}
\end{array}\right] .
$$

Thus, the conditional variance of $x_{t}$ is given by:

$$
\begin{gathered}
\operatorname{Var}\left[x_{t} \mid s_{t}=i\right]=\Omega(i)\left(\sum_{j=1}^{S} E\left[x_{t-1} x_{t-1}^{\prime} \mid s_{t-1}=j\right] \frac{P_{j}}{P_{i}} P_{j i}\right) \Omega^{\prime}(i)+ \\
\Gamma(i) \Xi_{z} \Gamma^{\prime}(i)+V(i) V^{\prime}(i),
\end{gathered}
$$

where $\Omega(i)=\Omega\left(S_{t}=i\right), \Gamma(i)=\Gamma\left(s_{t}=i\right)$ and $V(i)=V\left(S_{t}=i\right)$. In order to obtain a closed form expression for $\operatorname{Var}\left[x_{t} \mid s=i\right]$, define $v^{x}, v^{z}$ and $v$ as follows:

$$
v^{x}=\left[\begin{array}{c}
\operatorname{vec}\left(E\left[x_{t} x_{t}^{\prime} \mid s_{t}=1\right]\right) \\
\operatorname{vec}\left(E\left[x_{t} x_{t}^{\prime} \mid s_{t}=2\right]\right) \\
\vdots \\
\operatorname{vec}\left(E\left[x_{t} x_{t}^{\prime} \mid s_{t}=S\right]\right)
\end{array}\right], v^{z}=\left[\begin{array}{c}
\operatorname{vec}\left(\Gamma(1) \Xi_{z} \Gamma^{\prime}(1)\right) \\
\operatorname{vec}\left(\Gamma(2) \Xi_{z} \Gamma^{\prime}(2)\right) \\
\vdots \\
\operatorname{vec}\left(\Gamma(S) \Xi_{z} \Gamma^{\prime}(S)\right)
\end{array}\right], v=\left[\begin{array}{c}
\operatorname{vec}\left(V(1) V^{\prime}(1)\right) \\
\operatorname{vec}\left(V(2) V^{\prime}(2)\right) \\
\vdots \\
\operatorname{vec}\left(V(S) V^{\prime}(S)\right)
\end{array}\right] .
$$

Then, the equation for $\operatorname{Var}\left[x_{t} \mid s=i\right]$ for all $i=1,2, \ldots, S$ can be expressed as

$$
v^{x}=\Sigma^{\Omega} v^{x}+v^{z}+v,
$$


where the $(i, j)$-th element of the matrix $\Sigma^{\Omega}$ is given by:

$$
\Sigma^{\Omega}=\left[\frac{P_{j}}{P_{i}} P_{j i} \Omega(i) \otimes \Omega(i)\right]
$$

Therefore, $v^{x}=\left(I_{n^{2} S}-\Sigma^{\Omega}\right)^{-1}\left[v^{z}+v\right]$. By reshaping $v^{x}$ back into a matrix form, we have the formula for $\operatorname{Var}\left[x_{t} \mid s=i\right]$ for all $i=1,2, \ldots, S$. Using the conditional variances, we can obtain the unconditional variance which is given by

$$
\begin{aligned}
\operatorname{Var}\left(x_{t}\right) & =E\left(x_{t} x_{t}^{\prime}\right)=E\left(E\left[x_{t} x_{t}^{\prime} \mid s_{t}\right]\right) \\
& =\sum_{i=1}^{S} E\left[x_{t} x_{t}^{\prime} \mid s_{t}=i\right] P_{i} \\
& =\sum_{i=1}^{S} \operatorname{Var}\left[x_{t} \mid s_{t}=i\right] P_{i} .
\end{aligned}
$$




\section{B}

\section{Appendix to Chapter 2}

\section{B.1}

\section{Proof of Proposition 2.1}

Proposition B.1 (Proposition 2.1) Assume that the nominal interest rate zero lower bound is binding in the short run $\left(t \in\left[T_{0}, T_{\text {exit }}\right)\right)$ and that the following conditions hold:

(C1) $\Gamma_{\mu \sigma} \equiv(1-\mu)(1-\beta \mu)-\mu \kappa \sigma>0$,

$(C 2) \quad \Gamma_{b \sigma} \equiv(1-b)(1-\beta b)-b \kappa \sigma>0$,

(C3) $\Gamma_{\phi} \equiv \phi_{\pi}+\frac{(1-\beta)}{\kappa} \phi_{y}>1$,

(C4) $r_{S}^{e}<-\Theta_{i_{M}}\left(\bar{r}-i_{M}\right)-\Theta_{G_{S}} G_{S}-\Theta_{\tau_{S}^{I}} \tau_{S}^{I}-\Theta_{G_{M}} G_{M}-\Theta_{\tau_{M}^{I}} \tau_{M}^{I}$.

If there is a transitional state generated by the monetary authority keeping the nominal interest rate at $i_{M}=0$, or at an optimally chosen level $i_{M}=i_{M}^{*}$, after the crisis is over $\left(\forall t \in\left[T_{\text {exit }}, T_{M}\right)\right)$, solutions for output, inflation and the nominal interest rate in each state can be obtained backward as follows:

(i) In the long run $\left(\forall t>T_{M}\right)$, with $r_{t}^{e}=\bar{r}$, there is a locally unique bounded solution such that $i_{L}=\bar{r}$, with $Y_{L}=\pi_{L}=0$.

(ii) There is a locally unique bounded medium-run solution $\left(\forall t \in\left[T_{\text {exit }}, T_{M}\right)\right)$, with $r_{t}^{e}=\bar{r}$ and $i_{t}=i_{M}$, such that

$$
\begin{aligned}
& Y_{M}=\Omega_{Y_{M}, i_{M}}\left(\bar{r}-i_{M}\right)+\Omega_{Y_{M}, G_{M}} G_{M}+\Omega_{Y_{M}, \tau_{M}^{I}} \tau_{M}^{I} \\
& \pi_{M}=\Omega_{\pi_{M}, i_{M}}\left(\bar{r}-i_{M}\right)+\Omega_{\pi_{M}, G_{M}} G_{M}+\Omega_{\pi_{M}, \tau_{M}^{I}} \tau_{M}^{I} .
\end{aligned}
$$

(iii) In the short run $\left(\forall t \in\left[T_{0}, T_{\text {exit }}\right)\right)$, with $r_{t}^{e}=r_{S}^{e}$, there is a locally unique bounded solution, such that $i_{t}=0$ and

$$
\begin{aligned}
& Y_{S}=\Omega_{Y_{S}, r_{S}^{e}} r_{S}^{e}+\Omega_{Y_{S}, G_{S}} G_{S}+\Omega_{Y_{S}, \tau_{S}^{I}} \tau_{S}^{I}+ \\
& \Omega_{Y_{S}, i_{M}}\left(\bar{r}-i_{M}\right)+\Omega_{Y_{S}, G_{M}} G_{M}+\Omega_{Y_{S}, \tau_{M}^{I}} \tau_{M}^{I} \\
& \pi_{S}=\Omega_{\pi_{S}, r_{S}^{e}} r_{S}^{e}+\Omega_{\pi_{S}, G_{S}} G_{S}+\Omega_{\pi_{S}, \tau_{S}^{I}} \tau_{S}^{I}+ \\
& \Omega_{\pi_{S}, i_{M}}\left(\bar{r}-i_{M}\right)+\Omega_{\pi_{S}, G_{M}} G_{M}+\Omega_{\pi_{S}, \tau_{M}^{I}} \tau_{M}^{I}
\end{aligned}
$$


where the analytical expressions for the coefficients $\Omega_{i, j}, i \in\left\{Y_{S}, \pi_{S}, Y_{M}, \pi_{M}\right\}$ and $j \in\left\{r_{s}^{e}, i_{M}, G_{S}, \tau_{S}^{I}, G_{M}, \tau_{M}^{I}\right\}$ and $\Theta_{k}, k \in\left\{i_{M}, G_{S}, \tau_{S}^{I}, G_{M}, \tau_{M}^{I}\right\}$, are defined in the appendix B.1 and depend on the structural parameters.

The proof of this proposition is divided into three parts. First, we discuss determinacy of the solutions in each state. The second part is the derivation of analytical expressions for output and inflation allocations in each state. The last part is the derivation of condition $(C 4)$ that guarantees that the zero lower bound is binding in the short run.

Proof. (Proposition 2.1 - Part I - Determinacy) The derivation of the conditions for determinacy and existence of a solution in this model follows the generalization of the Taylor principle, presented by Davig \& Leeper (19) for a New Keynesian model with regime changes in monetary policy. The states in the economy are interpreted as different regimes, and it is possible to construct a probability transition matrix from the probability structure defined in the three-state economy set-up presented in Section 2.3.2. One aspect that makes this matrix simpler is the assumption that once the economy moves forward to the transitional state, or to the long run, it does not go back to the previous states.

Here it is assumed that the first state $\left(s_{t}=1\right)$ is the short run (crisis state), where the shock hits the economy $\left(r_{1}^{e}=r_{S}^{e}\right)$ and the zero lower bound is binding $\left(i_{1}=0\right)$. The second state $\left(s_{t}=2\right)$ is the medium run (transitional state), where the shock is no longer active $\left(r_{2}^{e}=\bar{r}\right)$, but the monetary authority keeps the nominal interest rate at a fixed value $\left(i_{2}=i_{M}\right)$ for a few periods. Finally, the third state $\left(s_{t}=3\right)$ is the long run $\left(r_{3}^{e}=\bar{r}\right)$, where the monetary policy goes back to following the rule (2-3) $\left(i_{3}=\bar{r}+\phi_{\pi} \pi_{t}+\phi_{y} Y_{t}\right)$.

The solutions are then defined by the set of equations formed, respectively, by the $(I S)$ equation (2-1), the aggregate supply relation (2-2) and the monetary policy rule (2-3).

$$
\begin{aligned}
\hat{Y}_{t} & =E_{t} \hat{Y}_{t+1}-\sigma\left(i_{t}-E_{t} \pi_{t+1}-r_{t}^{e}\right)+\left(\hat{G}_{t}-E_{t} \hat{G}_{t+1}\right) \\
\pi_{t} & =\kappa \hat{Y}_{t}+\kappa \psi\left(\chi^{I} \hat{\tau}_{t}^{I}-\sigma^{-1} \hat{G}_{t}\right)+\beta E_{t} \pi_{t+1} \\
0, & s_{t}=1 \text { (Short run) } \\
i_{M}, & \left.s_{t}=2 \text { (Medium run }\right) \\
i_{t} & =\left\{\begin{array}{r}
\text { (Long run) }
\end{array}\right\} .
\end{aligned}
$$

According to the diagram in Figure 2.1, we can set up the probability transition matrix: 


$$
\Pi \equiv\left[\begin{array}{ccc}
p_{11} & p_{12} & p_{13} \\
p_{21} & p_{22} & p_{23} \\
p_{31} & p_{32} & p_{33}
\end{array}\right]=\left[\begin{array}{ccc}
\mu & (1-\mu) b & (1-\mu)(1-b) \\
0 & b & (1-b) \\
0 & 0 & 1
\end{array}\right]
$$

The state-contingent expectations for output and inflation are given by

$$
\begin{aligned}
E_{t} \hat{Y}_{t+1} & =E\left[Y_{t+1} \mid s_{t}=j, \Omega_{t}^{-s}\right] \\
& =p_{j 1} E\left[Y_{1 t+1} \mid \Omega_{t}^{-s}\right]+p_{j 2} E\left[Y_{2 t+1} \mid \Omega_{t}^{-s}\right]+p_{j 3} E\left[Y_{3 t+1} \mid \Omega_{t}^{-s}\right] \\
E_{t} \pi_{t+1} & =E\left[\pi_{t+1} \mid s_{t}=j, \Omega_{t}^{-s}\right] \\
& =p_{j 1} E\left[\pi_{1 t+1} \mid \Omega_{t}^{-s}\right]+p_{j 2} E\left[\pi_{2 t+1} \mid \Omega_{t}^{-s}\right]+p_{j 3} E\left[\pi_{3 t+1} \mid \Omega_{t}^{-s}\right]
\end{aligned}
$$

where $j \in\{1,2,3\}$ and $\Omega_{t}^{-s}$ denotes agents' information set at time $t$, not including the current regime and $\Omega_{t}=\Omega_{t}^{-s} \cup\left\{s_{t}\right\}$.

Expectations regarding government spending are given by:

$$
E_{t} \hat{G}_{t+1}=E\left[G_{t+1} \mid s_{t}=j, \Omega_{t}^{-s}\right]=p_{j 1} G_{1}+p_{j 2} G_{2}+p_{j 3} G_{3}
$$

All expectations in B-1 and B-2 are formed conditional on $\Omega_{t}$. The $(I S)$ and $(A S)$ equations can be written as:

$$
\begin{aligned}
\left(I S_{j}\right) \quad Y_{j t}= & p_{j 1} E_{t} Y_{1 t+1}+p_{j 2} E_{t} Y_{2 t+1}+p_{j 3} E_{t} Y_{3 t+1}+ \\
& \sigma\left(p_{j 1} E_{t} \pi_{1 t+1}+p_{j 2} E_{t} \pi_{2 t+1}+p_{j 3} E_{t} \pi_{3 t+1}\right)+ \\
& \left(p_{j 1} G_{1}+p_{j 2} G_{2}+p_{j 3} G_{3}\right)-G_{j}-\sigma i_{j t}+\sigma r_{j t}^{e} \\
\left(A S_{j}\right) \quad \pi_{j t}= & \beta\left(p_{j 1} E_{t} \pi_{1 t+1}+p_{j 2} E_{t} \pi_{2 t+1}+p_{j 3} E_{t} \pi_{3 t+1}\right)+ \\
& \kappa Y_{j t}+\kappa \psi\left(\chi^{I} \tau_{j}^{I}-\sigma^{-1} G_{j}\right)
\end{aligned}
$$

Define state-contingent forecast errors for each state $j$ :

$$
\begin{aligned}
& \eta_{j t+1}^{\pi}=\pi_{j t+1}-E_{t} \pi_{j t+1} \Rightarrow E_{t} \pi_{j t+1}=\pi_{j t+1}-\eta_{j t+1}^{\pi} \\
& \eta_{j t+1}^{Y}=Y_{j t+1}-E_{t} Y_{j t+1} \Rightarrow E_{t} Y_{j t+1}=Y_{j t+1}-\eta_{j t+1}^{Y}
\end{aligned}
$$

and use them to eliminate the conditional expectations and rewrite $\left(I S_{j}\right)$ and $\left(A S_{j}\right)$ as: 


$$
\begin{aligned}
\left(I S_{j}\right) \quad Y_{j t}= & p_{j 1}\left(Y_{1 t+1}-\eta_{1 t+1}^{Y}\right)+p_{j 2}\left(Y_{2 t+1}-\eta_{2 t+1}^{Y}\right)+p_{j 3}\left(Y_{3 t+1}-\eta_{3 t+1}^{Y}\right)+ \\
& \sigma p_{j 1}\left(\pi_{1 t+1}-\eta_{1 t+1}^{\pi}\right)+\sigma p_{j 2}\left(\pi_{2 t+1}-\eta_{2 t+1}^{\pi}\right)+ \\
& \sigma p_{j 3}\left(\pi_{3 t+1}-\eta_{3 t+1}^{\pi}\right)+\left(p_{j 1} G_{1}+p_{j 2} G_{2}+p_{j 3} G_{3}\right) \\
& -G_{j}-\sigma i_{j t}+\sigma r_{j t}^{e}
\end{aligned}
$$

$$
\begin{aligned}
\left(A S_{j}\right) \pi_{j t}= & \beta p_{j 1}\left(\pi_{1 t+1}-\eta_{1 t+1}^{\pi}\right)+\beta p_{j 2}\left(\pi_{2 t+1}-\eta_{2 t+1}^{\pi}\right)+ \\
& \beta p_{j 3}\left(\pi_{3 t+1}-\eta_{3 t+1}^{\pi}\right)+\kappa Y_{j t}+\kappa \psi\left(\chi^{I} \tau_{j}^{I}-\sigma^{-1} G_{j}\right)
\end{aligned}
$$

Hence, we can write the system of equations for each state, incorporating the monetary policy stance into the $(I S)$ equation to get the aggregate demand relation. In the first state (short run) we have:

$$
\begin{aligned}
&\left(A D_{1}\right) p_{11} Y_{1 t+1}+p_{12} Y_{2 t+1}+p_{13} Y_{3 t+1}+\sigma\left(p_{11} \pi_{1 t+1}+p_{12} \pi_{2 t+1}+p_{13} \pi_{3 t+1}\right)= \\
& Y_{1 t}+\left(p_{11} \eta_{1 t+1}^{Y}+p_{12} \eta_{2 t+1}^{Y}+p_{13} \eta_{3 t+1}^{Y}\right)+ \\
& \sigma\left(p_{11} \eta_{1 t+1}^{\pi}+p_{12} \eta_{2 t+1}^{\pi}+p_{13} \eta_{3 t+1}^{\pi}\right)-\sigma r_{S}^{e} \\
&-\left[\left(p_{11}-1\right) G_{1}+p_{12} G_{2}+p_{13} G_{3}\right] \\
& \\
&\left(A S_{1}\right) \quad \beta\left(p_{11} \pi_{1 t+1}+p_{12} \pi_{2 t+1}+p_{13} \pi_{3 t+1}\right)=\pi_{1 t}-\kappa Y_{1 t}+ \\
& \beta\left(p_{11} \eta_{1 t+1}^{\pi}+p_{12} \eta_{2 t+1}^{\pi}+p_{13} \eta_{3 t+1}^{\pi}\right)-\kappa \psi\left(\chi^{I} \tau_{1}^{I}-\sigma^{-1} G_{1}\right)
\end{aligned}
$$

In the second state (medium run) we have:

$$
\begin{aligned}
\left(A D_{2}\right) & p_{21} Y_{1 t+1}+p_{22} Y_{2 t+1}+p_{23} Y_{3 t+1}+\sigma\left(p_{21} \pi_{1 t+1}+p_{22} \pi_{2 t+1}+p_{23} \pi_{3 t+1}\right)= \\
& Y_{2 t}+\left(p_{21} \eta_{1 t+1}^{Y}+p_{22} \eta_{2 t+1}^{Y}+p_{23} \eta_{3 t+1}^{Y}\right)+ \\
& \sigma\left(p_{21} \eta_{1 t+1}^{\pi}+p_{22} \eta_{2 t+1}^{\pi}+p_{23} \eta_{3 t+1}^{\pi}\right)-\sigma\left[\bar{r}-i_{M}\right] \\
& -\left[p_{21} G_{1}+\left(p_{22}-1\right) G_{2}+p_{23} G_{3}\right] \\
& \\
\left(A S_{2}\right) \quad & \beta\left(p_{21} \pi_{1 t+1}+p_{22} \pi_{2 t+1}+p_{23} \pi_{3 t+1}\right)=\pi_{2 t}-\kappa Y_{2 t}+ \\
& \beta\left(p_{21} \eta_{1 t+1}^{\pi}+p_{22} \eta_{2 t+1}^{\pi}+p_{23} \eta_{3 t+1}^{\pi}\right)-\kappa \psi\left(\chi^{I} \tau_{2}^{I}-\sigma^{-1} G_{2}\right)
\end{aligned}
$$

Finally, in the third state (long run) the system is given by:

$\left(A D_{3}\right) \quad p_{31} Y_{1 t+1}+p_{32} Y_{2 t+1}+p_{33} Y_{3 t+1}+\sigma\left(p_{31} \pi_{1 t+1}+p_{32} \pi_{2 t+1}+p_{33} \pi_{3 t+1}\right)=$ $\sigma \phi_{\pi} \pi_{3 t}+\left(1+\sigma \phi_{y}\right) Y_{3 t}+\left(p_{31} \eta_{1 t+1}^{Y}+p_{32} \eta_{2 t+1}^{Y}+p_{33} \eta_{3 t+1}^{Y}\right)+$ $\sigma\left(p_{31} \eta_{1 t+1}^{\pi}+p_{32} \eta_{2 t+1}^{\pi}+p_{33} \eta_{3 t+1}^{\pi}\right)-\left[p_{31} G_{1}+p_{32} G_{2}+\left(p_{33}-1\right) G_{3}\right]$

$\left(A S_{3}\right) \quad \beta\left(p_{31} \pi_{1 t+1}+p_{32} \pi_{2 t+1}+p_{33} \pi_{3 t+1}\right)=\pi_{3 t}-\kappa Y_{3 t}+$ $\beta\left(p_{31} \eta_{1 t+1}^{\pi}+p_{32} \eta_{2 t+1}^{\pi}+p_{33} \eta_{3 t+1}^{\pi}\right)-\kappa \psi\left(\chi^{I} \tau_{3}^{I}-\sigma^{-1} G_{3}\right)$ 
The complete system can be expressed in matrix form

$$
\begin{aligned}
& A X_{t+1}=B X_{t}+A \eta_{t}+C e_{t} \\
& X_{t} \equiv\left[\begin{array}{c}
\pi_{1 t} \\
Y_{1 t} \\
\pi_{2 t} \\
Y_{2 t} \\
\pi_{3 t} \\
Y_{3 t}
\end{array}\right] \quad \eta_{t} \equiv\left[\begin{array}{c}
\eta_{1 t+1}^{\pi} \\
\eta_{1 t+1}^{Y} \\
\eta_{2 t+1}^{\pi} \\
\eta_{2 t+1}^{Y} \\
\eta_{3 t+1}^{\pi} \\
\eta_{3 t+1}^{Y}
\end{array}\right] \quad e_{t} \equiv\left[\begin{array}{c}
r_{S}^{e} \\
i_{M} \\
\bar{r} \\
G_{1} \\
\tau_{1}^{I} \\
G_{2} \\
\tau_{2}^{I} \\
G_{3} \\
\tau_{3}^{I}
\end{array}\right] \\
& A=\Pi \otimes\left[\begin{array}{ll}
\beta & 0 \\
\sigma & 1
\end{array}\right] \quad B=\left[\begin{array}{cccccc}
1 & -\kappa & 0 & 0 & 0 & 0 \\
0 & 1 & 0 & 0 & 0 & 0 \\
0 & 0 & 1 & -\kappa & 0 & 0 \\
0 & 0 & 0 & 1 & 0 & 0 \\
0 & 0 & 0 & 0 & 1 & -\kappa \\
0 & 0 & 0 & 0 & \sigma \phi_{\pi} & \left(1+\sigma \phi_{y}\right)
\end{array}\right] \\
& C \equiv\left[\begin{array}{ccccccccc}
0 & 0 & 0 & \kappa \psi \sigma^{-1} & -\kappa \psi \chi^{I} & 0 & 0 & 0 & 0 \\
-\sigma & 0 & 0 & (1-\mu) & 0 & -(1-\mu) b & 0 & -(1-\mu)(1-b) & 0 \\
0 & 0 & 0 & 0 & 0 & \kappa \psi \sigma^{-1} & -\kappa \psi \chi^{I} & 0 & 0 \\
0 & \sigma & \sigma & 0 & 0 & (1-b) & 0 & -(1-b) & 0 \\
0 & 0 & 0 & 0 & 0 & 0 & 0 & \kappa \psi \sigma^{-1} & -\kappa \psi \chi^{I} \\
0 & 0 & 0 & 0 & 0 & 0 & 0 & 0 & 0
\end{array}\right]
\end{aligned}
$$

Since $A$ is invertible, we can write the system as:

$$
X_{t+1}=A^{-1} B X_{t}+\eta_{t}+A^{-1} C e_{t}
$$

where $A^{-1} B=\left[\begin{array}{ccc}Z_{11} & Z_{12} & 0_{2 \times 2} \\ 0_{2 \times 2} & Z_{22} & Z_{23} \\ 0_{2 \times 2} & 0_{2 \times 2} & Z_{33}\end{array}\right] ; \quad Z_{11} \equiv\left[\begin{array}{cc}\frac{1}{\beta \mu} & -\frac{\kappa}{\beta \mu} \\ -\frac{\sigma}{\beta \mu} & \frac{\beta+\kappa \sigma}{\beta \mu}\end{array}\right]$;

$$
\begin{aligned}
& Z_{12} \equiv\left[\begin{array}{cc}
-\frac{(1-\mu)}{\beta \mu} & \frac{\kappa(1-\mu)}{\beta \mu} \\
\frac{\sigma(1-\mu)}{\beta \mu} & -\frac{(\beta+\kappa \sigma)(1-\mu)}{\beta \mu}
\end{array}\right] ; \quad Z_{22} \equiv\left[\begin{array}{cc}
\frac{1}{\beta b} & -\frac{\kappa}{\beta b} \\
-\frac{\sigma}{\beta b} & \frac{\beta+\kappa \sigma}{\beta b}
\end{array}\right] ; \\
& Z_{23} \equiv\left[\begin{array}{cc}
-\frac{(1-b)}{\beta b} & \frac{\kappa(1-b)}{\beta b} \\
\frac{\left(1-\beta \phi_{\pi}\right) \sigma(1-b)}{\beta b} & -\frac{\left(\beta\left(1+\sigma \phi_{y}\right)+\kappa \sigma\right)(1-b)}{\beta b}
\end{array}\right] ; \\
& Z_{33} \equiv\left[\begin{array}{cc}
\frac{1}{\beta} & -\frac{\kappa}{\beta} \\
\frac{\sigma\left(\beta \phi_{\pi}-1\right)}{\beta} & \frac{\beta\left(1+\sigma \phi_{y}\right)+\kappa \sigma}{\beta}
\end{array}\right] ;
\end{aligned}
$$


The rational-expectations solution is determined if and only if matrix $A^{-1} B$ has all its eigenvalues outside the unit circle. Since this matrix is block lower triangular, its eigenvalues are those defined by the block matrices in the diagonal $\left(Z_{11}, Z_{22}, Z_{33}\right)$.

Hence, we can use the result from Proposition C.1 in Woodford (2) (Pgs. 670-71) to verify the determinacy conditions. This proposition states that the eigenvalues of a $2 \times 2$ matrix $Z$ are outside the unit circle if and only if one of the following two cases are satisfied:

Case I: $(a) \operatorname{det}(Z)>1$ and $(b) \operatorname{det}(Z)-\operatorname{tr}(Z)>-1$ and $(c) \operatorname{det}(Z)+\operatorname{tr}(Z)>-1$;

Case II: $(d) \operatorname{det}(Z)-\operatorname{tr}(Z)<-1$ and $(e) \operatorname{det}(Z)+\operatorname{tr}(Z)<-1$

So, I verify the conditions under which one of these cases are satisfied for each matrix $Z_{i i}$, reminding that all parameters $\left(\beta, \kappa, \sigma, \mu, b, \phi_{\pi}, \phi_{y}\right)$ are positive. Starting with $Z_{11}$ :

$$
\operatorname{tr}\left(Z_{11}\right)=\frac{1}{\beta \mu}+\left(1+\frac{\kappa \sigma}{\beta}\right) \frac{1}{\mu} \quad \operatorname{det}\left(Z_{11}\right)=\frac{1}{\beta \mu^{2}}
$$

Both conditions in Case II are clearly not satisfied since all parameters are positive. So I check conditions in Case I:

(a) $\operatorname{det}\left(Z_{11}\right)=\frac{1}{\beta \mu^{2}}>1$

(b) $\operatorname{det}\left(Z_{11}\right)-\operatorname{tr}\left(Z_{11}\right)>-1$

$$
\begin{aligned}
\operatorname{det}\left(Z_{11}\right)-\operatorname{tr}\left(Z_{11}\right) & =\frac{1}{\beta \mu^{2}}-\frac{1}{\beta \mu}-\frac{1}{\mu}-\frac{\kappa \sigma}{\beta \mu}>-1 \\
& \Rightarrow \beta \mu^{2}-\mu-\beta \mu-\mu \kappa \sigma+1>0 \\
& \Rightarrow \beta \mu^{2}-\beta \mu+1-\mu-\mu \kappa \sigma>0 \\
& \Rightarrow-(1-\mu) \beta \mu+1-\mu-\mu \kappa \sigma>0 \\
& \left.\Rightarrow \Gamma_{\mu \sigma} \equiv(1-\mu)(1-\beta \mu)-\mu \kappa \sigma>0 \quad \text { (Condition }(C 1)\right)
\end{aligned}
$$

(c) $\operatorname{det}\left(Z_{11}\right)+\operatorname{tr}\left(Z_{11}\right)>-1$

$$
\operatorname{det}\left(Z_{11}\right)+\operatorname{tr}\left(Z_{11}\right)=\frac{\beta^{-1}}{\mu^{2}}+\frac{1+\beta^{-1}(1+\kappa \sigma)}{\mu}>-1
$$

Thus, matrix $Z_{11}$ has both eigenvalues with modulus $|\lambda|>1$, if Condition $(C 1)$ holds. Matrix $Z_{22}$ is analogous to $Z_{11}$. We just need to switch $\mu$ for $b$ to get that its eigenvalues are outside the unit circle if the following condition holds: 


$$
\Gamma_{b \sigma} \equiv(1-b)(1-\beta b)-b \kappa \sigma>0
$$

For matrix $Z_{33}$ we have:

$$
\operatorname{tr}\left(Z_{33}\right)=\frac{1+\beta \sigma \phi_{y}+\kappa \sigma+\beta}{\beta} \quad \operatorname{det}\left(Z_{33}\right)=\frac{\sigma\left(\phi_{y}+\kappa \phi_{\pi}\right)+1}{\beta}
$$

Again, since all parameters are positive, the conditions in Case II are clearly not satisfied. So I verify the conditions in Case I:

$$
\begin{aligned}
& \text { (a) } \operatorname{det}\left(Z_{33}\right)=\frac{1+\sigma\left(\phi_{y}+\kappa \phi_{\pi}\right)}{\beta}>1 \\
& \begin{aligned}
\operatorname{lb} \operatorname{det}\left(Z_{33}\right)-\operatorname{tr}\left(Z_{33}\right) & >-1 \\
\operatorname{det}\left(Z_{33}\right)-\operatorname{tr}\left(Z_{33}\right) & =\frac{1+\sigma\left(\phi_{y}+\kappa \phi_{\pi}\right)-1-\beta \sigma \phi_{y}-\kappa \sigma-\beta}{\beta}>-1 \\
& \Rightarrow \sigma \kappa \phi_{\pi}+\sigma \phi_{y}-\beta \sigma \phi_{y}-\kappa \sigma-\beta>-\beta \\
& \Rightarrow \sigma \kappa \phi_{\pi}+(1-\beta \sigma) \phi_{y}-\kappa \sigma>0 \\
& \left.\Rightarrow \Gamma_{\phi} \equiv \kappa\left(\phi_{\pi}-1\right)+(1-\beta) \phi_{y}>0 \quad \text { (Condition }(C 3)\right)
\end{aligned}
\end{aligned}
$$

which holds since we assume $\phi_{\pi}>1$ and $\phi_{y}>0$.

(c) $\operatorname{det}\left(Z_{33}\right)+\operatorname{tr}\left(Z_{33}\right)>-1$

$$
\operatorname{det}\left(Z_{33}\right)+\operatorname{tr}\left(Z_{33}\right)=\frac{2+\sigma\left(\phi_{y}+\kappa \phi_{\pi}\right)+\beta \sigma \phi_{y}+\kappa \sigma+\beta}{\beta}>-1
$$

I should point out that Condition $(C 1)$ is necessary for the short-run solution to be defined and it does not depend on the fiscal measures taken. However, if the fiscal policy implemented during the crisis state is expected to last for a few periods after the crisis is over, while the monetary policy is expected to go back to the rule right after the zero lower bound is no longer binding, than we need Condition $(C 3)$ to be satisfied as well. In the case that the transitional state is generated by the monetary authority keeping the nominal interest rate fixed for a few periods after the crisis is over (independently if the fiscal stimulus is also maintained or not), Condition (C2) also needs to be satisfied to guarantee that the solution is determinate.

Proof. (Proposition 2.1 - Part II - Output and Inflation Allocations in Each State) All solutions in this model are obtained by solving the system formed by the $(I S)$ and the aggregate demand equations, besides the 
specifications for monetary and fiscal policies in each state.

$$
\begin{aligned}
(I S) & \hat{Y}_{t}=E_{t} \hat{Y}_{t+1}-\sigma\left(i_{t}-E_{t} \pi_{t+1}-r_{t}^{e}\right)+\left(\hat{G}_{t}-E_{t} \hat{G}_{t+1}\right) \\
(A S) & \pi_{t}=\kappa \hat{Y}_{t}+\kappa \psi\left(\chi^{I} \hat{\tau}_{t}^{I}-\sigma^{-1} \hat{G}_{t}\right)+\beta E_{t} \pi_{t+1}
\end{aligned}
$$

Given the expectations terms in these equations, to obtain the allocations in each state, it is necessary to solve for them backward. One should first compute long-run output and inflation, then use them to get the medium-run allocations and, finally, use both of them to obtain output and inflation in the short run. ${ }^{1}$

\section{Long-Run Solution Allocations}

I assume that, in the long run, the shock that hits the economy during the crisis state has already faded away $\left(r_{t}=\bar{r}, \forall t>T_{M}\right)$ and the zero lower bound is no longer binding. Policy instruments return to their steady-state values $\left(\hat{G}_{t}=\tau_{t}^{I}=0\right)$ bringing output, inflation and the nominal rate back to their long-run equilibrium as well ( $\hat{Y}_{t}=\pi_{t}=0$ and $i_{t}=\bar{r}$ ).

\section{Medium-Run Solution Allocations (Transitional State)}

The transitional-state allocations $\left(\pi_{M}, Y_{M}\right)$ are obtained by solving the system formed by $(I S)$ and $(A S)$ equations, given monetary and fiscal policies. But first it is necessary to get the expectations regarding future values of inflation, output and government spending that enter into these equations. Recall that once the economy enters the medium run $\left(t \in\left[T_{\text {exit }}, T_{M}\right)\right.$ and $b \neq 0$ ), in each following period there is probability $b$ that it stays there, while with probability $(1-b)$ it goes to the long-run allocation $\left(\pi_{L}=Y_{L}=G_{L}=0\right)$. Thus, expectations in the transitional state are given by:

$$
\begin{aligned}
E_{t} \hat{Y}_{t+1} & =b Y_{M}+(1-b) Y_{L}=b Y_{M} \\
E_{t} \pi_{t+1} & =b \pi_{M}+(1-b) \pi_{L}=b \pi_{M} \\
E_{t} \hat{G}_{t+1} & =b G_{M}+(1-b) G_{L}=b G_{M}
\end{aligned}
$$

Once the economy exits the crisis and the zero lower bound is no longer binding, the monetary authority can keep stimulating it by holding the nominal interest rate at a different level from that prescribed by rule B-3 for a few periods $\left(i_{M}\right)$. Hence, $i_{M}$ is kept in the aggregate demand equation to derive

${ }^{1}$ Given the linearity of the model, in the derivation of the solution allocations we assume that both fiscal instruments are used to obtain the full expressions for output and inflation. To discuss the impact of each instrument in Section 2.6, we shut down the other according to the policy that we want to analyze. 
the medium-run solution as a function of it, but later we make $\left(i_{M}=0\right)$ to compute the numerical solutions.

Using the expectations obtained above, with the value for the nominal interest rate and $\left(\pi_{L}=Y_{L}=G_{L}=0\right)$, the aggregate demand equation becomes:

$$
\begin{array}{rrr}
Y_{M}= & Y_{L}+\frac{\sigma}{(1-b)}\left(\bar{r}-i_{M}\right)+\frac{\sigma b}{(1-b)} \pi_{M}+G_{M} \\
Y_{M}= & b Y_{M}-\sigma\left(i_{M}-b \pi_{M}-\bar{r}\right)+\left(G_{M}-b G_{M}\right) \\
Y_{M}= & \frac{\sigma}{(1-b)}\left(\bar{r}-i_{M}\right)+\frac{b \sigma}{(1-b)} \pi_{M}+G_{M}
\end{array}
$$

while the aggregate supply is given by

$$
\begin{array}{lr}
\pi_{M}= & \kappa Y_{M}+\kappa \psi\left(\chi^{I} \tau_{M}^{I}-\sigma^{-1} G_{M}\right)+\beta b \pi_{M} \\
\pi_{M}= & \frac{\kappa}{(1-\beta b)} Y_{M}+\frac{\kappa \psi}{(1-\beta b)}\left(\chi^{I} \tau_{M}^{I}-\sigma^{-1} G_{M}\right)
\end{array}
$$

Substituting (B-7) into (B-8):

$$
\begin{aligned}
\pi_{M}= & \frac{\kappa}{(1-\beta b)}\left\{\frac{\sigma}{(1-b)}\left(\bar{r}-i_{M}\right)+\frac{b \sigma}{(1-b)} \pi_{M}+G_{M}\right\}+ \\
& \frac{\kappa \psi}{(1-\beta b)}\left(\chi^{I} \tau_{M}^{I}-\sigma^{-1} G_{M}\right) \\
\pi_{M} & =\frac{(1-b)}{(1-\beta b)(1-b)-b \kappa \sigma} \times \\
& \left\{\frac{\kappa \sigma}{(1-b)}\left(\bar{r}-i_{M}\right)+\left(1-\psi \sigma^{-1}\right) \kappa G_{M}+\kappa \psi \chi^{I} \tau_{M}^{I}\right\}
\end{aligned}
$$

Substitute back into (B-7):

$$
\begin{gathered}
Y_{M}=\frac{\sigma}{(1-b)}\left(\bar{r}-i_{M}\right)+G_{M}+ \\
\frac{b \sigma}{\Gamma_{b \sigma}}\left\{\frac{\kappa \sigma}{(1-b)}\left(\bar{r}-i_{M}\right)+\kappa \psi \chi^{I} \tau_{M}^{I}+\left(1-\psi \sigma^{-1}\right) \kappa G_{M}\right\} \\
Y_{M}=\frac{1}{\Gamma_{b \sigma}}\left\{(1-\beta b) \sigma\left(\bar{r}-i_{M}\right)+[(1-\beta b)(1-b)-b \kappa \psi] G_{M}+b \kappa \sigma \psi \chi^{I} \tau_{M}^{I}\right\}
\end{gathered}
$$

These results can be summarized as:

$$
Y_{M}=\Omega_{Y_{M}, i_{M}}\left(\bar{r}-i_{M}\right)+\Omega_{Y_{M}, G_{M}} G_{M}+\Omega_{Y_{M}, \tau_{M}^{I}} \tau_{M}^{I}
$$


where $\Omega_{Y_{M}, i_{M}} \equiv \frac{(1-\beta b) \sigma}{\Gamma_{b \sigma}}, \quad \Omega_{Y_{M}, G_{M}} \equiv \frac{[(1-\beta b)(1-b)-b \kappa \psi]}{\Gamma_{b \sigma}}$ and $\Omega_{Y_{M}, \tau_{M}^{I}} \equiv \frac{b \kappa \sigma \psi \chi^{I}}{\Gamma_{b \sigma}}$

$$
\pi_{M}=\Omega_{\pi_{M}, i_{M}}\left(\bar{r}-i_{M}\right)+\Omega_{\pi_{M}, G_{M}} G_{M}+\Omega_{\pi_{M}, \tau_{M}^{I}} \tau_{M}^{I}
$$

where $\Omega_{\pi_{M}, i_{M}} \equiv \frac{\kappa \sigma}{\Gamma_{b \sigma}}, \quad \Omega_{\pi_{M}, G_{M}} \equiv \frac{(1-b)\left(1-\psi \sigma^{-1}\right) \kappa}{\Gamma_{b \sigma}}$ and $\Omega_{\pi_{M}, \tau_{M}^{I}} \equiv$ $\frac{(1-b) \kappa \psi \chi^{I}}{\Gamma_{b \sigma}}$.

\section{Short-Run Solution Allocations (Crisis State)}

The last step is to solve for the short-run allocations $\left(\pi_{S}, Y_{S}\right)$ that characterize the crisis state. First, it is necessary to obtain expectations regarding future values of inflation, output and government spending that enter into the aggregate demand and supply equations. Recall that once the economy enters the crisis state at $t=T_{0}$, in each following period, there is a probability $\mu$ that it stays there, while with probability $(1-\mu) b$ it goes to the transitional state $\left(\pi_{M}, Y_{M}, G_{M}\right)$ and with probability $(1-\mu)(1-b)$ it goes straight to the long-run $\left(\pi_{L}, Y_{L}, G_{L}\right)$. Thus expectations are given by:

$$
\begin{aligned}
E_{t} \hat{Y}_{t+1} & =\mu Y_{S}+(1-\mu) b Y_{M}+(1-\mu)(1-b) Y_{L}=\mu Y_{S}+(1-\mu) b Y_{M} \\
E_{t} \pi_{t+1} & =\mu \pi_{S}+(1-\mu) b \pi_{M}+(1-\mu)(1-b) \pi_{L}=\mu \pi_{S}+(1-\mu) b \pi_{M} \\
E_{t} \hat{G}_{t+1} & =\mu G_{S}+(1-\mu) b G_{M}+(1-\mu)(1-b) G_{L}=\mu G_{S}+(1-\mu) b G_{M}
\end{aligned}
$$

The crisis state is characterized by a shock $\left(r_{t}^{e}=r_{S}^{e}\right)$ that makes the zero lower bound binding for the nominal interest rate $\left(i_{S}=0\right)$. So the aggregate demand equation is given by:

$$
\begin{aligned}
& Y_{S}=\mu Y_{S}+(1-\mu) b Y_{M}+(1-\mu)(1-b) Y_{L} \\
& \quad-\sigma\left(0-\left(\mu \pi_{S}+(1-\mu) b \pi_{M}+(1-\mu)(1-b) \pi_{L}\right)-r_{S}^{e}\right)+ \\
& \quad G_{S}-\left(\mu G_{S}+(1-\mu) b G_{M}+(1-\mu)(1-b) G_{L}\right)
\end{aligned}
$$

Using $\left(\pi_{L}=Y_{L}=G_{L}=0\right)$,

$$
Y_{S}=\frac{\sigma}{(1-\mu)} r_{S}^{e}+\frac{\mu \sigma}{(1-\mu)} \pi_{S}+b Y_{M}+b \sigma \pi_{M}+G_{S}-b G_{M}
$$

while the aggregate supply is given by:

$$
\begin{aligned}
& \pi_{S}=\kappa Y_{S}+\kappa \psi\left(\chi^{I} \tau_{S}^{I}-\sigma^{-1} G_{S}\right)+\beta \mu \pi_{S}+\beta(1-\mu) b \pi_{M}+\beta(1-\mu)(1-b) \pi_{L} \\
& \pi_{S}=\frac{\kappa}{(1-\beta \mu)} Y_{S}+\frac{\kappa \psi}{(1-\beta \mu)}\left(\chi^{I} \tau_{S}^{I}-\sigma^{-1} G_{S}\right)+\frac{\beta(1-\mu) b}{(1-\beta \mu)} \pi_{M}
\end{aligned}
$$


where the second line makes use of $\left(\pi_{L}=Y_{L}=G_{L}=0\right)$. Substituting (B-13) into (B-14):

$$
\begin{aligned}
\pi_{S}= & \frac{\kappa}{(1-\beta \mu)}\left\{\frac{\sigma}{(1-\mu)} r_{S}^{e}+\frac{\mu \sigma}{(1-\mu)} \pi_{S}+b Y_{M}+b \sigma \pi_{M}+G_{S}-b G_{M}\right\}+ \\
& \frac{\kappa \psi}{(1-\beta \mu)}\left(\chi^{I} \tau_{S}^{I}-\sigma^{-1} G_{S}\right)+\frac{\beta(1-\mu) b}{(1-\beta \mu)} \pi_{M} \\
\pi_{S}= & \frac{1}{\Gamma_{\mu \sigma}}\left\{\begin{array}{l}
\kappa \sigma r_{S}^{e}+(1-\mu)\left(1-\psi \sigma^{-1}\right) \kappa G_{S}+(1-\mu) \kappa \psi \chi^{I} \tau_{S}^{I}+ \\
(1-\mu) \kappa b\left(Y_{M}-G_{M}\right)+[\beta(1-\mu)+\kappa \sigma](1-\mu) b \pi_{M}
\end{array}\right\}
\end{aligned}
$$

Substitute back into (B-13):

$$
\begin{gathered}
Y_{S}=\frac{\sigma}{(1-\mu)} r_{S}^{e}+G_{S}+b\left(Y_{M}-G_{M}\right)+\sigma b \pi_{M}+ \\
\frac{\mu \sigma}{(1-\mu)} \frac{1}{\Gamma_{\mu \sigma}}\left\{\begin{array}{l}
\kappa \sigma r_{S}^{e}+(1-\mu) \kappa \psi \chi^{I} \tau_{S}^{I}+ \\
(1-\mu)\left(1-\psi \sigma^{-1}\right) \kappa G_{S}+ \\
(1-\mu) \kappa b\left(Y_{M}-G_{M}\right)+ \\
{[\beta(1-\mu)+\kappa \sigma](1-\mu) b \pi_{M}}
\end{array}\right\} \\
Y_{S}=\frac{1}{\Gamma_{\mu \sigma}}\left\{\begin{array}{l}
(1-\beta \mu) \sigma r_{S}^{e}+[(1-\beta \mu)(1-\mu)-\mu \kappa \psi] G_{S}+ \\
\mu \kappa \sigma \psi \chi^{I} \tau_{S}^{I}+(1-\beta \mu)(1-\mu) b\left(Y_{M}-G_{M}\right)+ \\
(1-\mu) b \sigma \pi_{M}
\end{array}\right\}
\end{gathered}
$$

Since we already solved for the medium-run allocations $\left(Y_{M}, \pi_{M}\right)$, we can use them in equations (B-15) and (B-16) to obtain the short-run solutions. But I want to express these relations in terms of the fiscal and monetary instruments used in each state. Thus, defining $\Gamma_{\mu \sigma} \equiv(1-\beta \mu)(1-\mu)-\mu \kappa \sigma$ and plugging the medium-run relations (B-11) and (B-12), into the crisis state output equation (B-16), we obtain:

$$
\begin{aligned}
Y_{S}= & \frac{1}{\Gamma_{\mu \sigma}}\left\{(1-\beta \mu) \sigma r_{S}^{e}+[(1-\beta \mu)(1-\mu)-\mu \kappa \psi] G_{S}+\mu \kappa \sigma \psi \chi^{I} \tau_{S}^{I}\right\}+ \\
& \frac{(1-\beta \mu)(1-\mu)}{\Gamma_{\mu \sigma}} b\left\{\begin{array}{l}
\Omega_{Y_{M}, i_{M}}\left(\bar{r}-i_{M}\right)+\Omega_{Y_{M}, G_{M}} G_{M}+ \\
\Omega_{Y_{M}}, \tau_{M^{I}} \tau_{M}^{I}-G_{M}
\end{array}\right\}+ \\
& \frac{(1-\mu) \sigma}{\Gamma_{\mu \sigma}}\left\{b\left[\Omega_{\pi_{M}, i_{M}}\left(\bar{r}-i_{M}\right)+\Omega_{\pi_{M}, G_{M}} G_{M}+\Omega_{\pi_{M}, \tau_{M}^{I}} \tau_{M}^{I}\right]\right\}
\end{aligned}
$$




$$
\begin{aligned}
Y_{S}= & \frac{(1-\beta \mu) \sigma}{\Gamma_{\mu \sigma}} r_{S}^{e}+\frac{[(1-\beta \mu)(1-\mu)-\mu \kappa \psi]}{\Gamma_{\mu \sigma}} G_{S}+\frac{\mu \kappa \sigma \psi \chi^{I}}{\Gamma_{\mu \sigma}} \tau_{S}^{I}+ \\
& \frac{(1-\mu) b\left[(1-\beta \mu) \Omega_{Y_{M}, i_{M}}+\sigma \Omega_{\pi_{M}, i_{M}}\right]}{\Gamma_{\mu \sigma}}\left(\bar{r}-i_{M}\right)+ \\
& \frac{(1-\mu) b}{\Gamma_{\mu \sigma}}\left[(1-\beta \mu)\left(\Omega_{Y_{M}, G_{M}}-1\right)+\sigma \Omega_{\pi_{M}, G_{M}}\right] G_{M}+ \\
& \frac{(1-\mu) b}{\Gamma_{\mu \sigma}}\left[(1-\beta \mu) \Omega_{Y_{M}, \tau_{M}^{I}}+\sigma \Omega_{\pi_{M}, \tau_{M}^{I}}\right] \tau_{M}^{I}
\end{aligned}
$$

which is summarized as:

$$
\begin{aligned}
& Y_{S}=\Omega_{Y_{S}, r_{S}^{e}} r_{S}^{e}+\Omega_{Y_{S}, G_{S}} G_{S}+\Omega_{Y_{S}, \tau_{S}^{I}} \tau_{S}^{I}+ \\
& \Omega_{Y_{S}, i_{M}}\left(\bar{r}-i_{M}\right)+\Omega_{Y_{S}, G_{M}} G_{M}+\Omega_{Y_{S}, \tau_{M}^{I}} \tau_{M}^{I}
\end{aligned}
$$

where $\Omega_{Y_{S}, r_{S}^{e}} \equiv \frac{(1-\beta \mu) \sigma}{\Gamma_{\mu \sigma}}, \Omega_{Y_{S}, G_{S}} \equiv \frac{[(1-\beta \mu)(1-\mu)-\mu \kappa \psi]}{\Gamma_{\mu \sigma}}$,

$\Omega_{Y_{S}, \tau_{S}^{I}} \equiv \frac{\mu \kappa \sigma \psi \chi^{I}}{\Gamma_{\mu \sigma}}, \Omega_{Y_{S}, i_{M}} \equiv \frac{(1-\mu) b}{\Gamma_{\mu \sigma}}\left[(1-\beta \mu) \Omega_{Y_{M}, i_{M}}+\sigma \Omega_{\pi_{M}, i_{M}}\right]$,

$\Omega_{Y_{S}, G_{M}} \equiv \frac{(1-\mu) b}{\Gamma_{\mu \sigma}}\left[(1-\beta \mu)\left(\Omega_{Y_{M}, G_{M}}-1\right)+\sigma \Omega_{\pi_{M}, G_{M}}\right]$ and

$\Omega_{Y_{S}, \tau_{M}^{I}} \equiv \frac{(1-\mu) b}{\Gamma_{\mu \sigma}}\left[(1-\beta \mu) \Omega_{Y_{M}, \tau_{M}^{I}}+\sigma \Omega_{\pi_{M}, \tau_{M}^{I}}\right]$.

Finally, plugging the medium-run relations (B-11) and (B-12), into the crisis-state inflation equation (B-15), we get:

$$
\begin{aligned}
\pi_{S}= & \frac{1}{\Gamma_{\mu \sigma}}\left\{\kappa \sigma r_{S}^{e}+(1-\mu)\left(1-\psi \sigma^{-1}\right) \kappa G_{S}+(1-\mu) \kappa \psi \chi^{I} \tau_{S}^{I}\right\} \\
& \frac{(1-\mu) b \kappa}{\Gamma_{\mu \sigma}}\left(\left[\Omega_{Y_{M}, i_{M}}\left(\bar{r}-i_{M}\right)+\Omega_{Y_{M}, G_{M}} G_{M}+\Omega_{Y_{M}, \tau_{M}^{I}} \tau_{M}^{I}\right]-G_{M}\right)+ \\
& \frac{[\beta(1-\mu)+\kappa \sigma](1-\mu) b}{\Gamma_{\mu \sigma}}\left[\Omega_{\pi_{M}, i_{M}}\left(\bar{r}-i_{M}\right)+\Omega_{\pi_{M}, G_{M}} G_{M}+\Omega_{\pi_{M}, \tau_{M}^{I}} \tau_{M}^{I}\right] \\
\pi_{S}= & \frac{\kappa \sigma}{\Gamma_{\mu \sigma}} r_{S}^{e}+\frac{(1-\mu)\left(1-\psi \sigma^{-1}\right) \kappa}{\Gamma_{\mu \sigma}} G_{S}+\frac{(1-\mu) \kappa \psi \chi^{I}}{\Gamma_{\mu \sigma}} \tau_{S}^{I}+ \\
& \frac{(1-\mu) b}{\Gamma_{\mu \sigma}}\left[\kappa \Omega_{Y_{M}, i_{M}}+[\beta(1-\mu)+\kappa \sigma] \Omega_{\pi_{M}, i_{M}}\right]\left(\bar{r}-i_{M}\right)+ \\
& \frac{(1-\mu) b}{\Gamma_{\mu \sigma}}\left[\kappa\left(\Omega_{Y_{M}, G_{M}}-1\right)+[\beta(1-\mu)+\kappa \sigma] \Omega_{\pi_{M}, G_{M}}\right] G_{M}+ \\
& \frac{(1-\mu) b}{\Gamma_{\mu \sigma}}\left[\kappa \Omega_{Y_{M}, \tau_{M}^{I}} \tau_{M}^{I}+[\beta(1-\mu)+\kappa \sigma] \Omega_{\left.\pi_{M}, \tau_{M}^{I}\right]}\right] \tau_{M}^{I}
\end{aligned}
$$

which can be summarized as:

$$
\begin{aligned}
& \pi_{S}=\Omega_{\pi_{S}, r_{S}^{e}} r_{S}^{e}+\Omega_{\pi_{S}, G_{S}} G_{S}+\Omega_{\pi_{S}, \tau_{S}^{I}} \tau_{S}^{I}+ \\
& \Omega_{\pi_{S}, i_{M}}\left(\bar{r}-i_{M}\right)+\Omega_{\pi_{S}, G_{M}} G_{M}+\Omega_{\pi_{S}, \tau_{M}^{I}} \tau_{M}^{I}
\end{aligned}
$$


where $\Omega_{\pi_{S}, r_{S}^{e}} \equiv \frac{\kappa \sigma}{\Gamma_{\mu \sigma}} r_{S}^{e}, \Omega_{\pi_{S}, G_{S}} \equiv \frac{(1-\mu)\left(1-\psi \sigma^{-1}\right) \kappa}{\Gamma_{\mu \sigma}}, \Omega_{\pi_{S}, \tau_{S}^{I}} \equiv \frac{(1-\mu) \kappa \psi \chi^{I}}{\Gamma_{\mu \sigma}}$,

$\Omega_{\pi_{S}, i_{M}} \equiv \frac{(1-\mu) b}{\Gamma_{\mu \sigma}}\left[\kappa \Omega_{Y_{M}, i_{M}}+[\beta(1-\mu)+\kappa \sigma] \Omega_{\pi_{M}, i_{M}}\right]$,

$\Omega_{\pi_{S}, G_{M}} \equiv \frac{(1-\mu) b}{\Gamma_{\mu \sigma}}\left[\kappa\left(\Omega_{Y_{M}, G_{M}}-1\right)+[\beta(1-\mu)+\kappa \sigma] \Omega_{\pi_{M}, G_{M}}\right]$ and

$\Omega_{\pi_{S}, \tau_{M}^{I}} \equiv \frac{(1-\mu) b}{\Gamma_{\mu \sigma}}\left[\kappa \Omega_{Y_{M}, \tau_{M}^{I}} \tau_{M}^{I}+[\beta(1-\mu)+\kappa \sigma] \Omega_{\pi_{M}, \tau_{M}^{I}}\right]$

Proof. (Proposition 2.1 - Part III - ZLB is binding) To find out the condition under which a shock $r_{t}^{e}=r_{S}^{e}$ makes the zero lower bound binding in the short run, we need to verify the combination of parameters and policy choices that would imply a negative nominal interest rate during the crisis if the monetary authority uses rule (B-3). Eggertsson(31) derives this condition under the assumption that once the shock is no longer active $\left(r_{t}^{e}=\bar{r}\right)$, the economy goes back to the long-run $\left(\hat{Y}_{t}=\pi_{t}=0\right)$.

However, I want to obtain a general condition that accounts for the effects that future expected policies have on short-run output and inflation. Hence I use the medium and long-run solution allocations derived in Part II of this proof.

It is then necessary to solve for the short-run allocations using the system formed by the $(I S)$ and $(A S)$ equations, together with the monetary policy rule:

$$
\begin{aligned}
(I S) \quad \hat{Y}_{t} & =E_{t} \hat{Y}_{t+1}-\sigma\left(i_{t}-E_{t} \pi_{t+1}-r_{t}^{e}\right)+\left(\hat{G}_{t}-E_{t} \hat{G}_{t+1}\right) \\
(A S) \quad \pi_{t} & =\kappa \hat{Y}_{t}+\kappa \psi\left(\chi^{I} \hat{\tau}_{t}^{I}-\sigma^{-1} \hat{G}_{t}\right)+\beta E_{t} \pi_{t+1} \\
(M P) & i_{t}=\max \left\{0, r_{t}^{e}+\phi_{\pi} \pi_{t}+\phi_{y} \hat{Y}_{t}\right\}
\end{aligned}
$$

given the expectations regarding future values of inflation, output and government spending:

$$
\begin{aligned}
E_{t} \hat{Y}_{t+1} & =\mu Y_{S}+(1-\mu) b Y_{M}+(1-\mu)(1-b) Y_{L}=\mu Y_{S}+(1-\mu) b Y_{M} \\
E_{t} \pi_{t+1} & =\mu \pi_{S}+(1-\mu) b \pi_{M}+(1-\mu)(1-b) \pi_{L}=\mu \pi_{S}+(1-\mu) b \pi_{M} \\
E_{t} \hat{G}_{t+1} & =\mu G_{S}+(1-\mu) b G_{M}+(1-\mu)(1-b) G_{L}=\mu G_{S}+(1-\mu) b G_{M}
\end{aligned}
$$

Substituting these expectations, the monetary policy rule and $\left(\pi_{L}=Y_{L}=G_{L}=0\right)$ into the $(I S)$ equation, we obtain the aggregate demand relation:

$$
\begin{aligned}
Y_{S}= & \mu Y_{S}+(1-\mu) b Y_{M}-\sigma\left(r_{S}^{e}+\phi_{\pi} \pi_{S}+\phi_{y} Y_{S}\right)+ \\
& \sigma\left(\mu \pi_{S}+(1-\mu) b \pi_{M}\right)+\sigma r_{S}^{e}+G_{S}-\left(\mu G_{S}+(1-\mu) b G_{M}\right)
\end{aligned}
$$




$$
\begin{aligned}
Y_{S}= & -\frac{\left(\phi_{\pi}-\mu\right)}{\left(1-\mu+\sigma \phi_{y}\right)} \sigma \pi_{S}+\frac{(1-\mu)}{\left(1-\mu+\sigma \phi_{y}\right)} G_{S}+ \\
& \frac{(1-\mu) b}{\left(1-\mu+\sigma \phi_{y}\right)}\left(Y_{M}-G_{M}\right)+\frac{(1-\mu) b \sigma}{\left(1-\mu+\sigma \phi_{y}\right)} \pi_{M}
\end{aligned}
$$

Doing the same for the aggregate supply equation:

$$
\begin{aligned}
& \pi_{S}=\kappa Y_{S}+\kappa \psi\left(\chi^{I} \tau_{S}^{I}-\sigma^{-1} G_{S}\right)+\beta\left(\mu \pi_{S}+(1-\mu) b \pi_{M}\right) \\
& \pi_{S}=\frac{\kappa}{(1-\beta \mu)} Y_{S}+\frac{\kappa \psi}{(1-\beta \mu)}\left(\chi^{I} \tau_{S}^{I}-\sigma^{-1} G_{S}\right)+\frac{\beta(1-\mu) b}{(1-\beta \mu)} \pi_{M}
\end{aligned}
$$

Substituting the aggregate demand into the aggregate supply, the shortrun inflation becomes:

$$
\begin{gathered}
\pi_{S}=\frac{\kappa}{(1-\beta \mu)}\left[\begin{array}{c}
-\frac{\left(\phi_{\pi}-\mu\right)}{\left(1-\mu+\sigma \phi_{y}\right)} \sigma \pi_{S}+\frac{(1-\mu)}{\left(1-\mu+\sigma \phi_{y}\right)} G_{S}+ \\
\frac{(1-\mu) b}{\left(1-\mu+\sigma \phi_{y}\right)}\left(Y_{M}-G_{M}\right)+\frac{(1-\mu) b \sigma}{\left(1-\mu+\sigma \phi_{y}\right)} \pi_{M}
\end{array}\right]+ \\
\frac{\kappa \psi}{(1-\beta \mu)}\left(\chi^{I} \tau_{S}^{I}-\sigma^{-1} G_{S}\right)+\frac{\beta(1-\mu) b}{(1-\beta \mu)} \pi_{M} \\
\pi_{S}=\frac{(1-\mu)\left(1-\psi \sigma^{-1}\right)-\phi_{y} \psi}{\Gamma_{\mu \phi}} \kappa G_{S}+ \\
\quad \frac{\left(1-\mu+\sigma \phi_{y}\right) \kappa \psi}{\Gamma_{\mu \phi}} \chi^{I} \tau_{S}^{I}+\frac{(1-\mu) \kappa}{\Gamma_{\mu \phi}} b\left(Y_{M}-G_{M}\right)+ \\
\quad \frac{(1-\mu)\left[\beta\left(1-\mu+\sigma \phi_{y}\right)+\kappa \sigma\right]}{\Gamma_{\mu \phi}} b \pi_{M}
\end{gathered}
$$

where $\Gamma_{\mu \phi} \equiv(1-\beta \mu)\left(1-\mu+\sigma \phi_{y}\right)+\left(\phi_{\pi}-\mu\right) \kappa \sigma$.

Plugging back into the aggregate demand equation, the expression for the short-run output becomes:

$$
\begin{aligned}
Y_{S}= & -\frac{\left(\phi_{\pi}-\mu\right)}{\left(1-\mu+\sigma \phi_{y}\right)} \sigma \times \\
& {\left[\begin{array}{l}
\frac{(1-\mu)\left(1-\psi \sigma^{-1}\right)-\phi_{y} \psi}{\Gamma_{\mu \phi}} \kappa G_{S}+\frac{\left(1-\mu+\sigma \phi_{y}\right) \kappa \psi}{\Gamma_{\mu \phi}} \chi^{I} \tau_{S}^{I}+ \\
\frac{(1-\mu) \kappa}{\Gamma_{\mu \phi}} b\left(Y_{M}-G_{M}\right)+\frac{(1-\mu)\left[\beta\left(1-\mu+\sigma \phi_{y}\right)+\kappa \sigma\right]}{\Gamma_{\mu \phi}} b \pi_{M}
\end{array}\right]+} \\
& \frac{(1-\mu)}{\left(1-\mu+\sigma \phi_{y}\right)} G_{S}+\frac{(1-\mu)}{\left(1-\mu+\sigma \phi_{y}\right)} b\left(Y_{M}-G_{M}\right)+\frac{(1-\mu) \sigma}{\left(1-\mu+\sigma \phi_{y}\right)} b \pi_{M}
\end{aligned}
$$




$$
\begin{aligned}
Y_{S}= & \frac{\left[(1-\mu)(1-\beta \mu)+\left(\phi_{\pi}-\mu\right) \kappa \psi\right]}{\Gamma_{\mu \phi}} G_{S} \\
& -\frac{\left(\phi_{\pi}-\mu\right) \kappa \sigma \psi}{\Gamma_{\mu \phi}} \chi^{I} \tau_{S}^{I}+\frac{(1-\beta \mu)(1-\mu)}{\Gamma_{\mu \phi}} b\left(Y_{M}-G_{M}\right)+ \\
& \frac{\left(1-\beta \phi_{\pi}\right)(1-\mu) \sigma}{\Gamma_{\mu \phi}} b \pi_{M}
\end{aligned}
$$

Plugging both short-run output and inflation into monetary policy rule (B-3), we have that the implied nominal interest rate is given by:

$$
\begin{aligned}
i_{S}= & r_{S}^{e}+\frac{(1-\mu)\left(1-\psi \sigma^{-1}\right) \phi_{\pi} \kappa+[(1-\mu)(1-\beta \mu)-\mu \kappa \psi] \phi_{y}}{\Gamma_{\mu \phi}} G_{S}+ \\
& \frac{(1-\mu) \phi_{\pi}+\mu \phi_{y} \sigma}{\Gamma_{\mu \phi}} \kappa \psi \chi^{I} \tau_{S}^{I}+\frac{(1-\mu)\left[\phi_{\pi} \kappa+(1-\beta \mu) \phi_{y}\right]}{\Gamma_{\mu \phi}} b\left(Y_{M}-G_{M}\right)+ \\
& \frac{(1-\mu)\left[\phi_{y} \sigma+(\beta(1-\mu)+\kappa \sigma) \phi_{\pi}\right]}{\Gamma_{\mu \phi}} b \pi_{M}
\end{aligned}
$$

Substituting the expressions for output and inflation in the medium run derived in Part II of this proof (equations (B-11) and (B-12)), I obtain that the nominal interest rate implied by the monetary policy rule is negative, and the zero lower bound is binding, if the shock respects the following condition:

$$
r_{S}^{e}<-\Theta_{G_{S}} G_{S}-\Theta_{\tau_{S}^{I}} \tau_{S}^{I}-\Theta_{\bar{r}}\left(\bar{r}-i_{M}\right)-\Theta_{G_{M}} G_{M}-\Theta_{\tau_{M}^{I}} \tau_{M}^{I}
$$

where the coefficients are given by

$$
\begin{aligned}
& \Theta_{G_{S}} \equiv \frac{(1-\mu)\left(1-\psi \sigma^{-1}\right) \phi_{\pi} \kappa+[(1-\mu)(1-\beta \mu)-\mu \kappa \psi] \phi_{y}}{\Gamma_{\mu \phi}}, \\
& \Theta_{\tau_{S}^{I}} \equiv \frac{(1-\mu) \phi_{\pi}+\mu \phi_{y} \sigma}{\Gamma_{\mu \phi}} \kappa \psi \chi^{I}, \Theta_{\bar{r}} \equiv \frac{(1-\mu) b}{\Gamma_{\mu \phi}}\left\{\begin{array}{c}
{\left[\phi_{\pi} \kappa+(1-\beta \mu) \phi_{y}\right] \Omega_{Y_{M}, i_{M}}+} \\
{\left[\phi_{y} \sigma+(\beta(1-\mu)+\kappa \sigma) \phi_{\pi}\right] \Omega_{\pi_{M}, i_{M}}}
\end{array}\right\}, \\
& \Theta_{G_{M}} \equiv \frac{(1-\mu) b}{\Gamma_{\mu \phi}}\left\{\begin{array}{c}
{\left[\phi_{\pi} \kappa+(1-\beta \mu) \phi_{y}\right]\left(\Omega_{Y_{M}, G_{M}}-1\right)+} \\
{\left[\phi_{y} \sigma+(\beta(1-\mu)+\kappa \sigma) \phi_{\pi}\right] \Omega_{\pi_{M}, G_{M}}}
\end{array}\right\}, \text { and } \\
& \Theta_{\tau_{M}^{I}} \equiv \frac{(1-\mu) b}{\Gamma_{\mu \phi}}\left\{\begin{array}{c}
{\left[\phi_{\pi} \kappa+(1-\beta \mu) \phi_{y}\right] \Omega_{Y_{M}, \tau_{M}^{I}+}+} \\
{\left[\phi_{y} \sigma+(\beta(1-\mu)+\kappa \sigma) \phi_{\pi}\right] \Omega_{\pi_{M}, \tau_{M}^{I}}^{I}}
\end{array}\right\}
\end{aligned}
$$




\section{B.2}

\section{Proof of Proposition 2.2}

Proposition B.2 (Proposition 2.2) Assume that the nominal interest rate zero lower bound is binding in the short run $\left(t \in\left[T_{0}, T_{\text {exit }}\right)\right)$ and that the following conditions hold:

(C1) $\quad \Gamma_{\mu \sigma} \equiv(1-\mu)(1-\beta \mu)-\mu \kappa \sigma>0$,

(C2) $\quad \Gamma_{b \sigma} \equiv(1-b)(1-\beta b)-b \kappa \sigma>0$,

(C3) $\Gamma_{\phi} \equiv \phi_{\pi}+\frac{(1-\beta)}{\kappa} \phi_{y}>1$,

$\left(C 4^{\prime}\right) \quad r_{S}^{e}<-\Theta_{G_{S}} G_{S}-\Theta_{\tau_{S}^{I}} \tau_{S}^{I}-\Theta_{G_{M}}^{T} G_{M}-\Theta_{\tau_{M}^{I}}^{T} \tau_{M}^{I}$.

If there is a transitional state generated by the fiscal authority keeping the stimulus provided in the short run for a few periods after the crisis is over $\left(\forall t \in\left[T_{\text {exit }}, T_{M}\right)\right)$, while monetary policy returns to rule (B-3) as soon as $r_{t}^{e}$ returns to $\bar{r}$, the solutions in each state can be obtained backward as follows:

(i) In the long run, there is a locally unique bounded solution $\left(\forall t>T_{M}\right)$, with $r_{t}^{e}=\bar{r}$, such that $i_{L}=\bar{r}$ and $Y_{L}=\pi_{L}=0$.

(ii) There is a locally unique bounded medium-run solution $\left(\forall t \in\left[T_{\text {exit }}, T_{M}\right)\right)$, with $r_{t}^{e}=\bar{r}$, such that

$$
\begin{aligned}
Y_{M}^{T} & =\Omega_{Y_{M}, G_{M}}^{T} G_{M}+\Omega_{Y_{M}, \tau_{M}^{I}}^{T} \tau_{M}^{I}, \\
\pi_{M}^{T} & =\Omega_{\pi_{M}, G_{M}}^{T} G_{M}+\Omega_{\pi_{M}, \tau_{M}^{I}}^{T} \tau_{M}^{I}, \\
i_{M}^{T} & =\bar{r}+\phi_{\pi} \pi_{M}^{T}+\phi_{y} Y_{M}^{T} .
\end{aligned}
$$

(iii) In the short run $\left(\forall t \in\left[T_{0}, T_{\text {exit }}\right)\right)$, with $r_{t}^{e}=r_{S}^{e}$, there is a locally unique bounded solution, such that $i_{s}=0$ and

$$
\begin{aligned}
& Y_{S}^{T}=\Omega_{Y_{S}, r_{S}^{e}} r_{S}^{e}+\Omega_{Y_{S}, G_{S}} G_{S}+\Omega_{Y_{S}, \tau_{S}^{I}} \tau_{S}^{I}+ \\
& \Omega_{Y_{S}, G_{M}}^{T} G_{M}+\Omega_{Y_{S}, \tau_{M}^{I}}^{T} \tau_{M}^{I} \\
& \pi_{S}^{T}=\Omega_{\pi_{S}, r_{S}^{e}}^{e}+\Omega_{\pi_{S}, G_{S}}^{e} G_{S}+\Omega_{\pi_{S}, \tau_{S}^{I}} \tau_{S}^{I}+ \\
& \Omega_{\pi_{S}, G_{M}}^{T} G_{M}+\Omega_{\pi_{S}, \tau_{M}^{I}}^{T} \tau_{M}^{I}
\end{aligned}
$$

where the analytical expressions for the coefficients $\Omega_{i, j}, i \in\left\{Y_{S}, \pi_{S}\right\}$ and $j \in\left\{r_{S}^{e}, G_{S}, \tau_{S}^{I}\right\}$, and $\Theta_{k}, k \in\left\{G_{S}, \tau_{S}^{I}\right\}$, are the same as those defined in Proposition 1. The expressions for the coefficients $\Omega_{m, n}^{T}, m \in\left\{Y_{S}, \pi_{S}, Y_{M}, \pi_{M}\right\}$ and $n \in\left\{G_{M}, \tau_{M}^{I}\right\}$, and $\Theta_{h}^{T}, k \in\left\{G_{M}, \tau_{M}^{I}\right\}$ are defined in the appendix and depend on the structural parameters.

The proof of this proposition follows the same procedure adopted for Proposition 1. It is divided into three parts. The first part discusses solutions' 
determinacy in each state. The second part derives the allocations for output and inflation in each state. Finally, the third part derives the condition under which the zero lower bound is binding in the short run.

Proof. (Proposition 2.2 - Part I - Determinacy) The derivation of the conditions for determinacy and existence of a solution in this model is analogous to that presented in Proposition 1. We will have the crisis state $\left(s_{t}=1\right)$, where the shock hits the economy $\left(r_{1}^{e}=r_{S}^{e}\right)$ and the zero lower bound is binding $\left(i_{1}=0\right)$, and the transitional state $\left(s_{t}=2\right)$, where the shock is no longer active $\left(r_{2}^{e}=\bar{r}\right)$, but the fiscal authority keeps the stimulus implemented during the crisis, while monetary policy goes back to following the rule $\left(i_{2}=\bar{r}+\phi_{\pi} \pi_{2}+\phi_{x} Y_{2}\right)$. In the long run $\left(s_{t}=3\right)$, fiscal instruments return to their steady states $\left(\hat{G}_{t}=\hat{\tau}_{t}^{I}=0, \forall t>T_{M}\right)$ implying $Y_{L}=\pi_{L}=0$, which simplifies the derivations.

The solutions is then defined by the set of equations formed, respectively, by the equation (B-1), the aggregate supply relation (B-2) and the monetary policy rule (B-3):

$$
\begin{aligned}
& \hat{Y}_{t}=E_{t} \hat{Y}_{t+1}-\sigma\left(i_{t}-E_{t} \pi_{t+1}-r_{t}^{e}\right)+\left(\hat{G}_{t}-E_{t} \hat{G}_{t+1}\right) \\
& \pi_{t}=\kappa \hat{Y}_{t}+\kappa \psi\left(\chi^{I} \hat{\tau}_{t}^{I}-\sigma^{-1} \hat{G}_{t}\right)+\beta E_{t} \pi_{t+1} \\
& 0, \quad s_{t}=1 \text { (Short run) } \\
& i_{t}=\left\{\begin{array}{r}
\left.\phi_{t} \text { (Medium run }\right) \\
\bar{r}+\phi_{\pi} \pi_{2}+\phi_{y} Y_{2}, \quad s_{t}=2 \text { (Long run) }
\end{array}\right\}
\end{aligned}
$$

The probability transition matrix is the same as in (B-4):

$$
\Pi \equiv\left[\begin{array}{ccc}
p_{11} & p_{12} & p_{13} \\
p_{21} & p_{22} & p_{23} \\
p_{31} & p_{32} & p_{33}
\end{array}\right]=\left[\begin{array}{ccc}
\mu & (1-\mu) b & (1-\mu)(1-b) \\
0 & b & (1-b) \\
0 & 0 & 1
\end{array}\right]
$$

The state-contingent expectations with respect to output and inflation are given by:

$$
\begin{aligned}
& E_{t} \hat{Y}_{t+1}=E\left[Y_{t+1} \mid s_{t}=j, \Omega_{t}^{-s}\right]=p_{j 1} E\left[Y_{1 t+1} \mid \Omega_{t}^{-s}\right]+p_{j 2} E\left[Y_{2 t+1} \mid \Omega_{t}^{-s}\right] \\
& E_{t} \pi_{t+1}=E\left[\pi_{t+1} \mid s_{t}=j, \Omega_{t}^{-s}\right]=p_{j 1} E\left[\pi_{1 t+1} \mid \Omega_{t}^{-s}\right]+p_{j 2} E\left[\pi_{2 t+1} \mid \Omega_{t}^{-s}\right]
\end{aligned}
$$

Expectations regarding government spending are given by:

$$
E_{t} \hat{G}_{t+1}=E\left[G_{t+1} \mid s_{t}=j, \Omega_{t}^{-s}\right]=p_{j 1} G_{1}+p_{j 2} G_{2}
$$


The $(I S)$ and $(A S)$ equations can be written as:

$$
\begin{aligned}
\left(I S_{j}\right) \quad Y_{j t}= & p_{j 1} E_{t} Y_{1 t+1}+p_{j 2} E_{t} Y_{2 t+1}+\sigma\left(p_{j 1} E_{t} \pi_{1 t+1}+p_{j 2} E_{t} \pi_{2 t+1}\right)+ \\
& \left(p_{j 1} G_{1}+p_{j 2} G_{2}\right)-G_{j}-\sigma i_{j t}+\sigma r_{j t}^{e} \\
\left(A S_{j}\right) \quad \pi_{j t}= & \beta\left(p_{j 1} E_{t} \pi_{1 t+1}+p_{j 2} E_{t} \pi_{2 t+1}\right)+\kappa Y_{j t}+\kappa \psi\left(\chi^{I} \tau_{j}^{I}-\sigma^{-1} G_{j}\right)
\end{aligned}
$$

where we already used $E_{t} Y_{3 t+1}=E_{t} \pi_{3 t+1}=0$. Define state-contingent forecast errors for each state $j$ :

$$
\begin{aligned}
& \eta_{j t+1}^{\pi}=\pi_{j t+1}-E_{t} \pi_{j t+1} \Rightarrow E_{t} \pi_{j t+1}=\pi_{j t+1}-\eta_{j t+1}^{\pi} \\
& \eta_{j t+1}^{Y}=Y_{j t+1}-E_{t} Y_{j t+1} \Rightarrow E_{t} Y_{j t+1}=Y_{j t+1}-\eta_{j t+1}^{Y}
\end{aligned}
$$

and use them to eliminate the conditional expectations and rewrite $\left(I S_{j}\right)$ and $\left(A S_{j}\right)$ as:

$$
\begin{aligned}
\left(I S_{j}\right) \quad Y_{j t}= & p_{j 1}\left(Y_{1 t+1}-\eta_{1 t+1}^{Y}\right)+p_{j 2}\left(Y_{2 t+1}-\eta_{2 t+1}^{Y}\right)+ \\
& \sigma\left(p_{j 1}\left(\pi_{1 t+1}-\eta_{1 t+1}^{\pi}\right)+p_{j 2}\left(\pi_{2 t+1}-\eta_{2 t+1}^{\pi}\right)\right)+ \\
& \left(p_{j 1} G_{1}+p_{j 2} G_{2}\right)-G_{j}-\sigma i_{j t}+\sigma r_{j t}^{e} \\
\left(A S_{j}\right) \quad \pi_{j t}= & \beta p_{j 1}\left(\pi_{1 t+1}-\eta_{1 t+1}^{\pi}\right)+\beta p_{j 2}\left(\pi_{2 t+1}-\eta_{2 t+1}^{\pi}\right)+\kappa Y_{j t}+ \\
& \kappa \psi\left(\chi^{I} \tau_{j}^{I}-\sigma^{-1} G_{j}\right)
\end{aligned}
$$

Hence, we can write the system of equations for each state, incorporating the monetary policy stance into the $(I S)$ equation to get the aggregate demand relation. In the first state (short run) we have:

$$
\begin{aligned}
\left(A D_{1}\right) & p_{11} Y_{1 t+1}+p_{12} Y_{2 t+1}+\sigma\left(p_{11} \pi_{1 t+1}+p_{12} \pi_{2 t+1}\right)=Y_{1 t}+ \\
& \left(p_{11} \eta_{1 t+1}^{Y}+p_{12} \eta_{2 t+1}^{Y}\right)+\sigma\left(p_{11} \eta_{1 t+1}^{\pi}+p_{12} \eta_{2 t+1}^{\pi}\right) \\
& -\sigma r_{S}^{e}-\left[\left(p_{11}-1\right) G_{1}+p_{12} G_{2}\right] \\
\left(A S_{1}\right) \quad \beta\left(p_{11} \pi_{1 t+1}+p_{12} \pi_{2 t+1}\right)=\pi_{1 t}-\kappa Y_{1 t}+\beta\left(p_{11} \eta_{1 t+1}^{\pi}+p_{12} \eta_{2 t+1}^{\pi}\right) & -\kappa \psi\left(\chi^{I} \tau_{1}^{I}-\sigma^{-1} G_{1}\right)
\end{aligned}
$$


In the second state (medium run) we have:

$\left(A D_{2}\right) \quad p_{21} Y_{1 t+1}+p_{22} Y_{2 t+1}+\sigma\left(p_{21} \pi_{1 t+1}+p_{22} \pi_{2 t+1}\right)=\sigma \phi_{\pi} \pi_{2 t}+$

$$
\begin{aligned}
& \left(1+\sigma \phi_{y}\right) Y_{2 t}+\left(p_{21} \eta_{1 t+1}^{Y}+p_{22} \eta_{2 t+1}^{Y}\right)+ \\
& \sigma\left(p_{21} \eta_{1 t+1}^{\pi}+p_{22} \eta_{2 t+1}^{\pi}\right)-\left[p_{21} G_{1}+\left(p_{22}-1\right) G_{2}\right]
\end{aligned}
$$

$\left(A S_{2}\right) \quad \beta\left(p_{21} \pi_{1 t+1}+p_{22} \pi_{2 t+1}\right)=\pi_{2 t}-\kappa Y_{2 t}+\beta\left(p_{21} \eta_{1 t+1}^{\pi}+p_{22} \eta_{2 t+1}^{\pi}\right)$

$$
-\kappa \psi\left(\chi^{I} \tau_{2}^{I}-\sigma^{-1} G_{2}\right)
$$

The complete system can be expressed in matrix form

$$
\begin{gathered}
A X_{t+1}=B X_{t}+A \eta_{t}+C e_{t} \\
X_{t} \equiv\left[\begin{array}{c}
\pi_{1 t} \\
Y_{1 t} \\
\pi_{2 t} \\
Y_{2 t}
\end{array}\right] \quad \eta_{t} \equiv\left[\begin{array}{c}
\eta_{1 t+1}^{\pi} \\
\eta_{1 t+1}^{Y} \\
\eta_{2 t+1}^{\pi} \\
\eta_{2 t+1}^{Y}
\end{array}\right] \quad e_{t} \equiv\left[\begin{array}{c}
r_{S}^{e} \\
G_{1} \\
\tau_{1}^{I} \\
G_{2} \\
\tau_{2}^{I}
\end{array}\right] \\
A=\left[\begin{array}{ccccc}
\mu & (1-\mu) b \\
0 & b
\end{array}\right] \otimes\left[\begin{array}{cc}
\beta & 0 \\
\sigma & 1
\end{array}\right] \\
\quad B=\left[\begin{array}{cccc}
1 & -\kappa & 0 & 0 \\
0 & 1 & 0 & 0 \\
0 & 0 & 1 & -\kappa \\
0 & 0 & \sigma \phi_{\pi} & \left(1+\sigma \phi_{y}\right)
\end{array}\right] \\
C \equiv\left[\begin{array}{ccccc}
0 & \\
-\sigma & (1-\mu) & 0 & -(1-\mu) b & 0 \\
0 & 0 & 0 & \kappa \psi \sigma^{-1} & -\kappa \psi \chi^{I} \\
0 & 0 & 0 & (1-b) & 0
\end{array}\right]
\end{gathered}
$$

Since $A$ is invertible, we can write the system as:

$$
X_{t+1}=A^{-1} B X_{t}+\eta_{t}+A^{-1} C e_{t}
$$

where $A^{-1} B=\left[\begin{array}{cc}Z_{11} & Z_{12} \\ 0_{2 \times 2} & Z_{22}\end{array}\right] ; Z_{11} \equiv\left[\begin{array}{cc}\frac{1}{\beta \mu} & -\frac{\kappa}{\beta \mu} \\ -\frac{\sigma}{\beta \mu} & \frac{\beta+\kappa \sigma}{\beta \mu}\end{array}\right]$;

$$
\begin{aligned}
& Z_{12} \equiv\left[\begin{array}{cc}
-\frac{(1-\mu)}{\beta \mu} & \frac{(1-\mu) \kappa}{\beta \mu} \\
\frac{\sigma(1-\mu)\left(1-\beta \phi_{\pi}\right)}{\beta \mu} & -\frac{(1-\mu)\left[\beta\left(1+\sigma \phi_{y}\right)+\kappa \sigma\right]}{\beta \mu}
\end{array}\right] \\
& Z_{22} \equiv\left[\begin{array}{cc}
\frac{1}{\beta b} & -\frac{\kappa}{\beta b} \\
\frac{\left(\beta \phi_{\pi}-1\right)}{\beta b} & \frac{\beta\left(1+\sigma \phi_{y}\right)+\kappa \sigma}{\beta b}
\end{array}\right]
\end{aligned}
$$


Again, we can check if the eigenvalues of matrix $A^{-1} B$ are outside the unit circle by analyzing the eigenvalues of the block matrices $Z_{i i}$ in the diagonal.

Matrix $Z_{11}$ is the same from the proof of Part I of Proposition 1. Thus we need Condition $(C 1)$ to hold to the short-run solution to be determinate. We can then use the result in Proposition C.1 from Woodford (2) to verify the determinacy conditions for matrix $Z_{22}$, reminding that all parameters $\left(\beta, \kappa, \sigma, \mu, b, \phi_{\pi}, \phi_{y}\right)$ are positive. For matrix $Z_{22}$ we have:

$$
\operatorname{tr}\left(Z_{22}\right)=\frac{1+\beta\left(1+\sigma \phi_{y}\right)+\kappa \sigma}{\beta b} \quad \operatorname{det}\left(Z_{22}\right)=\frac{1+\sigma\left(\phi_{y}+\kappa \phi_{\pi}\right)}{\beta b^{2}}
$$

Since all parameters are positive, the conditions in Case II of Woodford's proposition are not satisfied. So I verify the conditions in Case I:

$(a) \operatorname{det}\left(Z_{22}\right)=\frac{1+\sigma\left(\phi_{y}+\kappa \phi_{\pi}\right)}{\beta b^{2}}>1$

(b) $\operatorname{det}\left(Z_{22}\right)-\operatorname{tr}\left(Z_{22}\right)>-1$

$$
\begin{aligned}
\operatorname{det}\left(Z_{22}\right)-\operatorname{tr}\left(Z_{22}\right) & =\frac{1+\sigma\left(\phi_{y}+\kappa \phi_{\pi}\right)}{\beta b^{2}}-\frac{1+\beta\left(1+\sigma \phi_{y}\right)+\kappa \sigma}{\beta b}>-1 \\
& \Rightarrow[(1-b)(1-\beta b)-b \kappa \sigma]+\left[(1-\beta b) \sigma \phi_{y}+\kappa \sigma \phi_{\pi}\right]>0
\end{aligned}
$$

The first term in this expression is the one from Condition $(C 2)$ which imposes it should be bigger than zero. The second part is similar to Condition $(C 3)$, but not the same. But given that $\phi_{\pi}>1$ and $\phi_{y}>0, \kappa>0, \beta \in[0,1]$ and $b \in[0,1]$, we have that the second term is also positive. In fact, it is easy to see that (Condition $(C 3))$ guarantees $\left[(1-\beta b) \sigma \phi_{y}+\kappa \sigma \phi_{\pi}\right]>0$. Thus, if Conditions $(C 2)$ and $(C 3)$ hold, then $\left[\operatorname{det}\left(Z_{22}\right)-\operatorname{tr}\left(Z_{22}\right)>-1\right]$.

(c) $\operatorname{det}\left(Z_{33}\right)+\operatorname{tr}\left(Z_{33}\right)>-1$

$$
\operatorname{det}\left(Z_{33}\right)+\operatorname{tr}\left(Z_{33}\right)=\frac{b+(1+\beta b)\left(1+\sigma \phi_{y}\right)+\left(\phi_{\pi}+b\right) \kappa \sigma}{\beta b^{2}}>-1
$$

Hence, the conditions for Case I are satisfied for matrix $Z_{22}$ and we need Conditions $(C 1)-(C 3)$ to hold to guarantee that the solution is determinate.

\section{Proof. (Proposition 2.2 - Part II - Output and Inflation Allocations}

in Each State) Again we need to obtain solutions for output and inflation in each state, working backward from the long-run to the short-run allocations. As was done in Part II of the proof of Proposition 1, we need to solve the system formed by the $(I S)$ and $(A S)$ equations, together with the specifications for 
monetary and fiscal policies in each state. The long-run solution is given by $i_{L}=\bar{r}$ and $Y_{L}=\pi_{L}=0$. So we only need to solve for the short-run and medium-run allocations.

\section{Medium-Run Solution Allocations (Transitional State)}

Given that the monetary authority follows the rule (B-24) in the transitional state, and the zero lower bound is no longer binding, the medium-run nominal interest rate is given by $i_{M}^{T}=\bar{r}+\phi_{\pi} \pi_{M}^{T}+\phi_{y} Y_{M}^{T}$, where $\left(Y_{M}^{T}, \pi_{M}^{T}\right)$ represent output and inflation in the transitional state under this rule. Expectations in the medium run are given by

$$
\begin{aligned}
E_{t} \hat{Y}_{t+1} & =b Y_{M}^{T}+(1-b) \times Y_{L}=b Y_{M}^{T} \\
E_{t} \pi_{t+1} & =b \pi_{M}^{T}+(1-b) \times \pi_{L}=b \pi_{M}^{T} \\
E_{t} \hat{G}_{t+1} & =b G_{M}+(1-b) \times G_{L}=b G_{M}
\end{aligned}
$$

Using these expectations and the monetary policy rule into the $(I S)$ equation, the aggregate demand equation becomes:

$$
\begin{gathered}
Y_{M}^{T}=b Y_{M}^{T}+\left(G_{M}-b G_{M}\right)-\sigma\left(\bar{r}+\phi_{\pi} \pi_{M}^{T}+\phi_{y} Y_{M}^{T}-b \pi_{M}^{T}-\bar{r}\right) \\
\left(1-b+\sigma \phi_{y}\right) Y_{M}^{T}=-\left(\phi_{\pi}-b\right) \sigma \pi_{M}^{T}+(1-b) G_{M} \\
Y_{M}^{T}=-\frac{\left(\phi_{\pi}-b\right)}{\left(1-b+\sigma \phi_{y}\right)} \sigma \pi_{M}^{T}+\frac{(1-b)}{\left(1-b+\sigma \phi_{y}\right)} G_{M}
\end{gathered}
$$

while the aggregate supply is given by

$$
\pi_{M}^{T}=\frac{\kappa}{(1-\beta b)} Y_{M}^{T}+\frac{\kappa \psi}{(1-\beta b)}\left(\chi^{I} \tau_{M}^{I}-\sigma^{-1} G_{M}\right)
$$

Substituting the aggregate demand expression into the aggregate supply:

$$
\begin{gathered}
\pi_{M}^{T}=\frac{\kappa}{(1-\beta b)}\left[-\frac{\left(\phi_{\pi}-b\right)}{\left(1-b+\sigma \phi_{y}\right)} \sigma \pi_{M}^{T}+\frac{(1-b)}{\left(1-b+\sigma \phi_{y}\right)} G_{M}\right]+ \\
\frac{\kappa \psi}{(1-\beta b)}\left(\chi^{I} \tau_{M}^{I}-\sigma^{-1} G_{M}\right) \\
\pi_{M}^{T}=\frac{1}{\Gamma_{b \phi}}\left\{\left[(1-b)\left(1-\psi \sigma^{-1}\right)-\psi \phi_{y}\right] \kappa G_{M}+\left(1-b+\sigma \phi_{y}\right) \kappa \psi \chi^{I} \tau_{M}^{I}\right\}
\end{gathered}
$$

where $\Gamma_{b \phi} \equiv(1-\beta b)\left(1-b+\sigma \phi_{y}\right)+\left(\phi_{\pi}-b\right) \kappa \sigma$. 
Substituting back into the aggregate demand equation:

$$
\begin{aligned}
& Y_{M}^{T}=-\frac{\left(\phi_{\pi}-b\right) \sigma}{\left(1-b+\sigma \phi_{y}\right)} \frac{1}{\Gamma_{b \phi}}\left\{\begin{array}{l}
{\left[(1-b)\left(1-\psi \sigma^{-1}\right)-\psi \phi_{y}\right] \kappa G_{M}+} \\
\left(1-b+\sigma \phi_{y}\right) \kappa \psi \chi^{I} \tau_{M}^{I}
\end{array}\right\}+ \\
& \frac{(1-b)}{\left(1-b+\sigma \phi_{y}\right)} G_{M} \\
& Y_{M}^{T}=\frac{1}{\Gamma_{b \phi}}\left\{\begin{array}{l}
{\left[(1-b)(1-\beta b)+\left(\phi_{\pi}-b\right) \kappa \psi\right] G_{M}+} \\
-\left(\phi_{\pi}-b\right) \kappa \sigma \psi \chi^{I} \tau_{M}^{I}
\end{array}\right\}
\end{aligned}
$$

The medium-run solution allocations can be summarized as follows:

$$
Y_{M}^{T}=\Omega_{Y_{M}, G_{M}}^{T} G_{M}+\Omega_{Y_{M}, \tau_{M}^{I}}^{T} \tau_{M}^{I}
$$

where $\Omega_{Y_{M}, G_{M}}^{T} \equiv \frac{(1-b)(1-\beta b)+\left(\phi_{\pi}-b\right) \kappa \psi}{\Gamma_{b \phi}}$ and

$\Omega_{Y_{M}, \tau_{M}^{I}}^{T} \equiv-\frac{\left(\phi_{\pi}-b\right) \kappa \sigma \psi \chi^{I}}{\Gamma_{b \phi}}$

$\pi_{M}^{T}=\Omega_{\pi_{M}, G_{M}}^{T} G_{M}+\Omega_{\pi_{M}, \tau_{M}^{I}}^{T} \tau_{M}^{I}$

where $\Omega_{\pi_{M}, G_{M}}^{T} \equiv \frac{\left[(1-b)\left(1-\psi \sigma^{-1}\right)-\psi \phi_{y}\right] \kappa}{\Gamma_{b \phi}}$ and

$\Omega_{\pi_{M}, \tau_{M}^{I}}^{T} \equiv \frac{\left(1-b+\sigma \phi_{y}\right) \kappa \psi \chi^{I}}{\Gamma_{b \phi}}$

\section{Short-Run Solution Allocations (Crisis State)}

Now we can solve for the short-run allocations. First, we need to compute expectations during the crisis state:

$$
\begin{aligned}
E_{t} \hat{Y}_{t+1} & =\mu Y_{S}^{T}+(1-\mu) b Y_{M}^{T}+(1-\mu)(1-b) \times Y_{L}=\mu Y_{S}^{T}+(1-\mu) b Y_{M}^{T} \\
E_{t} \pi_{t+1} & =\mu \pi_{S}^{T}+(1-\mu) b \pi_{M}^{T}+(1-\mu)(1-b) \times \pi_{L}=\mu \pi_{S}^{T}+(1-\mu) b \pi_{M}^{T} \\
E_{t} \hat{G}_{t+1} & =\mu G_{S}+(1-\mu) b G_{M}+(1-\mu)(1-b) \times G_{L}=\mu G_{S}+(1-\mu) b G_{M}
\end{aligned}
$$

The solution for output and inflation in the crisis state is analogous to equations (B-15) and (B-16) from Part II of Proposition 1, substituting $Y_{L}=\pi_{L}=0$ :

$$
\begin{gathered}
Y_{S}^{T}=\frac{1}{\Gamma_{\mu \sigma}}\left\{\begin{array}{c}
(1-\beta \mu) \sigma r_{S}^{e}+[(1-\beta \mu)(1-\mu)-\mu \kappa \psi] G_{S}+\mu \kappa \sigma \psi \chi^{I} \tau_{S}^{I}+ \\
(1-\beta \mu)(1-\mu) b\left(Y_{M}-G_{M}\right)+(1-\mu) \sigma b \pi_{M}
\end{array}\right\} \\
\pi_{S}^{T}=\frac{1}{\Gamma_{\mu \sigma}}\left\{\begin{array}{l}
\kappa \sigma r_{S}^{e}+(1-\mu)\left(1-\psi \sigma^{-1}\right) \kappa G_{S}+(1-\mu) \kappa \psi \chi^{I} \tau_{S}^{I} \\
(1-\mu) \kappa b\left(Y_{M}-G_{M}\right)+[\beta(1-\mu)+\kappa \sigma](1-\mu) b \pi_{M}
\end{array}\right\}
\end{gathered}
$$


Substituting the medium-run solution allocations (B-28) and (B-29) into these short-run relations, we obtain crisis-state output and inflation as a function of the fiscal instruments, which can be summarized as:

$$
\begin{aligned}
Y_{S}^{T}=\Omega_{Y_{S}, r_{S}^{e}} r_{S}^{e} & +\Omega_{Y_{S}, G_{S}} G_{S}+\Omega_{Y_{S}, \tau_{S}^{I}} \tau_{S}^{I} \\
& +\Omega_{Y_{S}, G_{M}}^{T} G_{M}+\Omega_{Y_{S}, \tau_{M}^{I}}^{T} \tau_{M}^{I}
\end{aligned}
$$

where $\Omega_{Y_{S}, r_{S}^{e}} \equiv \frac{(1-\beta \mu) \sigma}{\Gamma_{\mu \sigma}}, \Omega_{Y_{S}, G_{S}} \equiv \frac{[(1-\beta \mu)(1-\mu)-\mu \kappa \psi]}{\Gamma_{\mu \sigma}}$,

$\Omega_{Y_{S}, \tau_{S}^{I}} \equiv \frac{\mu \kappa \sigma \psi \chi^{I}}{\Gamma_{\mu \sigma}}, \Omega_{Y_{S}, G_{M}}^{T} \equiv \frac{(1-\mu) b}{\Gamma_{\mu \sigma}}\left[(1-\beta \mu)\left(\Omega_{Y_{M}, G_{M}}^{T}-1\right)+\sigma \Omega_{\pi_{M}, G_{M}}^{T}\right]$ and $\Omega_{Y_{S}, \tau_{M}^{I}}^{T} \equiv \frac{(1-\mu) b}{\Gamma_{\mu \sigma}}\left[(1-\beta \mu) \Omega_{Y_{M}, \tau_{M}^{I}}^{T}+\sigma \Omega_{\pi_{M}, \tau_{M}^{I}}^{T}\right]$

$$
\begin{aligned}
\pi_{S}^{T}=\Omega_{\pi_{S}, r_{S}^{e}} r_{S}^{e} & +\Omega_{\pi_{S}, G_{S}} G_{S}+\Omega_{\pi_{S}, \tau_{S}^{I}} \tau_{S}^{I} \\
& +\Omega_{\pi_{S}, G_{M}}^{T} G_{M}+\Omega_{\pi_{S}, \tau_{M}^{I}}^{T} \tau_{M}^{I}
\end{aligned}
$$

where $\Omega_{\pi_{S}, r_{S}^{e}} \equiv \frac{\kappa \sigma}{\Gamma_{\mu \sigma}} r_{S}^{e}, \Omega_{\pi_{S}, G_{S}} \equiv \frac{(1-\mu)\left(1-\psi \sigma^{-1}\right) \kappa}{\Gamma_{\mu \sigma}}, \Omega_{\pi_{S}, \tau_{S}^{I}} \equiv \frac{(1-\mu) \kappa \psi \chi^{I}}{\Gamma_{\mu \sigma}}$, $\Omega_{\pi_{S}, G_{M}}^{T} \equiv \frac{(1-\mu) b}{\Gamma_{\mu \sigma}}\left[\kappa\left(\Omega_{Y_{M}, G_{M}}^{T}-1\right)+[\beta(1-\mu)+\kappa \sigma] \Omega_{\pi_{M}, G_{M}}^{T}\right]$ and $\Omega_{\pi_{S}, \tau_{M}^{I}}^{T} \equiv \frac{(1-\mu) b}{\Gamma_{\mu \sigma}}\left[\kappa \Omega_{Y_{M}, \tau_{M}^{I}}^{T} \tau_{M}^{I}+[\beta(1-\mu)+\kappa \sigma] \Omega_{\pi_{M}, \tau_{M}^{I}}^{T}\right]$.

Proof. (Proposition 2.2 - Part III - ZLB is binding) With the new allocations defined for output and inflation in the short and medium runs, we need to derive again the relation between the shock and the fiscal instruments that guarantee that the zero lower bound is binding in the short run (Condition $\left(C 4^{\prime}\right)$ ). The idea follows that used to derive Condition $(C 4)$ in Proposition 1, remembering that the long-run allocation is given by $Y_{L}=\pi_{L}=0$. We can already use the result from equation (B-21) and substitute for output and inflation in the transitional state ((B-28) and (B-29)) to obtain:

$$
\begin{aligned}
i_{S}= & r_{S}^{e}+\frac{(1-\mu)\left(1-\psi \sigma^{-1}\right) \phi_{\pi} \kappa+[(1-\mu)(1-\beta \mu)-\mu \kappa \psi] \phi_{y}}{\Gamma_{\mu \phi}} G_{S}+ \\
& \frac{(1-\mu) \phi_{\pi}+\mu \phi_{y} \sigma}{\Gamma_{\mu \phi}} \kappa \psi \chi^{I} \tau_{S}^{I}+ \\
& \frac{(1-\mu)\left[\phi_{\pi} \kappa+(1-\beta \mu) \phi_{y}\right]}{\Gamma_{\mu \phi}} b\left(\left(\Omega_{Y_{M}, G_{M}}^{T}-1\right) G_{M}+\Omega_{Y_{M}, \tau_{M}^{I}}^{T} \tau_{M}^{I}\right)+ \\
& \frac{(1-\mu)\left[\phi_{y} \sigma+(\beta(1-\mu)+\kappa \sigma) \phi_{\pi}\right]}{\Gamma_{\mu \phi}} b\left(\Omega_{\pi_{M}, G_{M}}^{T} G_{M}+\Omega_{\pi_{M}, \tau_{M}^{I}}^{T} \tau_{M}^{I}\right)
\end{aligned}
$$




$$
\begin{aligned}
& i_{S}=r_{S}^{e}+\frac{(1-\mu)\left(1-\psi \sigma^{-1}\right) \phi_{\pi} \kappa+[(1-\mu)(1-\beta \mu)-\mu \kappa \psi] \phi_{y}}{\Gamma_{\mu \phi}} G_{S}+ \\
& \frac{(1-\mu) \phi_{\pi}+\mu \phi_{y} \sigma}{\Gamma_{\mu \phi}} \kappa \psi \chi^{I} \tau_{S}^{I}+ \\
& \frac{(1-\mu) b}{\Gamma_{\mu \phi}}\left[\begin{array}{l}
{\left[\phi_{\pi} \kappa+(1-\beta \mu) \phi_{y}\right]\left(\Omega_{Y_{M}, G_{M}}^{T}-1\right)+} \\
{\left[\phi_{y} \sigma+(\beta(1-\mu)+\kappa \sigma) \phi_{\pi}\right] \Omega_{\pi_{M}, G_{M}}^{T}}
\end{array}\right] G_{M}+ \\
& \frac{(1-\mu) b}{\Gamma_{\mu \phi}}\left[\begin{array}{l}
{\left[\phi_{\pi} \kappa+(1-\beta \mu) \phi_{y}\right] \Omega_{Y_{M}, \tau_{M}^{I}}^{T}+} \\
{\left[\phi_{y} \sigma+(\beta(1-\mu)+\kappa \sigma) \phi_{\pi}\right] \Omega_{\pi_{M}, \tau_{M}^{I}}^{T}}
\end{array}\right] \tau_{M}^{I}
\end{aligned}
$$

Thus, the nominal interest rate implied by the monetary policy rule is negative, and the zero lower bound is binding, if the shock respects the following condition:

$$
r_{S}^{e}<-\Theta_{G_{S}} G_{S}-\Theta_{\tau_{S}^{I}} \tau_{S}^{I}-\Theta_{G_{M}}^{T} G_{M}-\Theta_{\tau_{M}^{I}}^{T} \tau_{M}^{I} \quad \text { (Condition }\left(C 4^{\prime}\right) \text { ) }
$$

where the coefficients are $\Theta_{G_{S}}$ and $\Theta_{\tau_{S}^{I}}$ are the same as those defined in Part III of the proof of Proposition 1 and the other coefficients are given

$$
\begin{aligned}
& \text { by } \Theta_{G_{M}}^{T} \equiv \frac{(1-\mu) b}{\Gamma_{\mu \phi}}\left\{\begin{array}{c}
{\left[\phi_{\pi} \kappa+(1-\beta \mu) \phi_{y}\right]\left(\Omega_{Y_{M}, G_{M}}^{T}-1\right)+} \\
{\left[\phi_{y} \sigma+(\beta(1-\mu)+\kappa \sigma) \phi_{\pi}\right] \Omega_{\pi_{M}, G_{M}}^{T}}
\end{array}\right\} \text { and } \\
& \Theta_{\tau_{M}^{I}}^{T} \equiv \frac{(1-\mu) b}{\Gamma_{\mu \phi}}\left\{\begin{array}{l}
{\left[\phi_{\pi} \kappa+(1-\beta \mu) \phi_{y}\right] \Omega_{Y_{M}, \tau_{M}^{I}}^{T}+} \\
{\left[\phi_{y} \sigma+(\beta(1-\mu)+\kappa \sigma) \phi_{\pi}\right] \Omega_{\pi_{M}, \tau_{M}^{I}}^{T}}
\end{array}\right\} .
\end{aligned}
$$




\section{C \\ Appendix to Chapter 3}

\section{C.1}

\section{Proof of Proposition 3.1}

Proposition C.1 (Proposition 3.1) The welfare loss function in this model is given by

$$
L_{t} \equiv \sum_{t=0}^{\infty} \beta^{t}\left\{\pi_{t}^{2}+\lambda_{y}\left(\hat{Y}_{t}-\Gamma \hat{G}_{t}\right)^{2}+\lambda_{g} \hat{G}_{t}^{2}\right\}
$$

where $\Gamma \equiv \frac{\sigma^{-1} \gamma}{\sigma^{-1}+\omega}, \lambda_{y} \equiv \frac{\kappa}{\theta}$ and $\lambda_{g} \equiv \lambda_{y} \Gamma(1-\gamma-\Gamma)$. Given the probability structure of the model, this function can be expressed in present discounted terms as

$$
L^{P D V}=\left\{\begin{array}{l}
\frac{1}{1-\beta \mu}\left(\pi_{S}^{2}+\lambda_{y}\left(Y_{S}-\Gamma G_{S}\right)^{2}+\lambda_{g} G_{S}^{2}\right)+ \\
\frac{\beta(1-\mu) b}{(1-\beta \mu)(1-\beta b)}\left(\pi_{M}^{2}+\lambda_{y}\left(Y_{M}-\Gamma G_{M}\right)^{2}+\lambda_{g} G_{M}^{2}\right)+ \\
\frac{\beta(1-\mu)(1-b)}{(1-\beta)(1-\beta \mu)(1-\beta b)}\left(\pi_{L}^{2}+\lambda_{y}\left(Y_{L}-\Gamma G_{L}\right)^{2}+\lambda_{g} G_{L}^{2}\right)
\end{array}\right\}
$$

The proof of this proposition is divided into two parts. In the first part, I derive the expression for the welfare loss function, while the second part shows how I obtain the expression for the present discounted value of the loss function.

Proof. (Proposition 3.1 - Part I - Welfare Loss Function) The welfare loss function is obtained through a second order Taylor expansion of the utility function, according to Woodford (2) and Eggertsson(44). The utility function in this model is given by:

$$
E\left\{\sum_{t=0}^{\infty} \beta^{t}\left[u\left(C_{t}, \xi_{t}\right)+g\left(G_{t}, \xi_{t}\right)-\int_{0}^{1} v\left(h_{t}(i), \xi_{t}\right) d i\right]\right\}
$$

I use the aggregate resource constraint $\left(Y_{t}=C_{t}+G_{t}\right)$ to substitute out for consumption and the production function $\left(y_{t}(i)=h_{t}(i)\right)$ to substitute out for labor. The utility function becomes

$$
E\left\{\sum_{t=0}^{\infty} \beta^{t}\left[u\left(Y_{t}-G_{t}, \xi_{t}\right)+g\left(G_{t}, \xi_{t}\right)-\int_{0}^{1} v\left(y_{t}(i), \xi_{t}\right) d i\right]\right\}
$$


The Taylor expansion is performed in each term of the utility function separately. The first term yields:

$$
\begin{aligned}
u\left(Y_{t}-G_{t}, \xi_{t}\right) \approx & \bar{u}+u_{c} \tilde{Y}_{t}-u_{c} \tilde{G}_{t}+u_{\xi} \xi_{t}+\frac{1}{2} u_{c c} \tilde{Y}_{t}^{2}-\frac{1}{2} u_{c c} \tilde{G}_{t}^{2}+ \\
& u_{c \xi} \xi_{t} \tilde{Y}_{t}-u_{c c} \tilde{Y}_{t} \tilde{G}_{t}-u_{c \xi} \xi_{t} \tilde{G}_{t}+\frac{1}{2} \xi_{t}^{\prime} u_{\xi \xi} \xi_{t}+\mathcal{O}\left(\|\xi\|^{3}\right)
\end{aligned}
$$

where $\bar{u} \equiv u(\bar{Y} ; 0), \tilde{Y}_{t} \equiv Y_{t}-\bar{Y}$ and $\tilde{G}_{t} \equiv G_{t}-\bar{G}$ and assuming that the fluctuations in $\tilde{Y}_{t}$ are of order $\mathcal{O}(\|\xi\|)$. This can be written as:

$$
\begin{aligned}
u\left(Y_{t}-G_{t}, \xi_{t}\right) \approx & \bar{u}+u_{c} \bar{Y}\left(\hat{Y}_{t}+\frac{1}{2} \hat{Y}_{t}^{2}\right)-u_{c} \bar{G}\left(\hat{G}_{t}+\frac{1}{2} \hat{G}_{t}^{2}\right)+u_{\xi} \xi_{t} \\
& +\frac{1}{2} u_{c c} \bar{Y}^{2}\left(\hat{Y}_{t}+\frac{1}{2} \hat{Y}_{t}^{2}\right)^{2}-\frac{1}{2} u_{c c} \bar{G}^{2}\left(\hat{G}_{t}+\frac{1}{2} \hat{G}_{t}^{2}\right)^{2} \\
& +u_{c \xi} \xi_{t} \bar{Y}\left(\hat{Y}_{t}+\frac{1}{2} \hat{Y}_{t}^{2}\right)-u_{c c} \bar{Y} \bar{G}\left(\hat{Y}_{t}+\frac{1}{2} \hat{Y}_{t}^{2}\right)\left(\hat{G}_{t}+\frac{1}{2} \hat{G}_{t}^{2}\right) \\
& -u_{c \xi} \xi_{t} \bar{G}\left(\hat{G}_{t}+\frac{1}{2} \hat{G}_{t}^{2}\right)+\frac{1}{2} \xi_{t}^{\prime} u_{\xi \xi} \xi_{t}+\mathcal{O}\left(\|\xi\|^{3}\right) \\
= & \bar{u}+u_{c} \bar{Y}\left(\hat{Y}_{t}+\frac{1}{2} \hat{Y}_{t}^{2}\right)-u_{c} \bar{G}\left(\hat{G}_{t}+\frac{1}{2} \hat{G}_{t}^{2}\right)+u_{\xi} \xi_{t} \\
& +\frac{1}{2} u_{c c} \bar{Y}^{2} \hat{Y}_{t}^{2}-\frac{1}{2} u_{c c} \bar{G}^{2} \hat{G}_{t}^{2}+u_{c \xi} \bar{Y} \xi_{t} \hat{Y}_{t}-u_{c c} \bar{Y} \hat{G}_{t} \hat{G}_{t} \\
& -u_{c \xi} \bar{G} \xi_{t} \hat{G}_{t}+\frac{1}{2} \tilde{\xi}_{t}^{\prime} u_{\xi \xi} \tilde{\xi}_{t}+\mathcal{O}\left(\|\xi\|^{3}\right)
\end{aligned}
$$

where I substitute $\tilde{Y}_{t}$ in terms of $\hat{Y}_{t} \equiv \log \left(Y_{t} / \bar{Y}\right)$, using the Taylor series expansion

$$
\frac{Y_{t}}{\bar{Y}}=1+\hat{Y}_{t}+\frac{1}{2} \hat{Y}_{t}^{2}+\mathcal{O}\left(\|\xi\|^{3}\right)
$$

Analogously, I substituted $\tilde{G}_{t}$ in terms of $\hat{G}_{t} \equiv \log \left(G_{t} / \bar{G}\right)$. This can be conveniently written as:

$$
\begin{aligned}
u\left(Y_{t}-G_{t}, \xi_{t}\right)= & u_{c} \bar{Y}\left[\hat{Y}_{t}+\frac{1}{2} \hat{Y}_{t}^{2}+\frac{1}{2} \frac{u_{c c} \bar{Y}}{u_{c}} \hat{Y}_{t}^{2}+\frac{u_{c \xi} \xi_{t}}{u_{c}} \hat{Y}_{t}\right] \\
& -u_{c} \bar{G}\left[\hat{G}_{t}+\frac{1}{2} \hat{G}_{t}^{2}+\frac{1}{2} \frac{u_{c c} \bar{Y}}{u_{c}} \overline{\bar{Y}} \hat{G}_{t}^{2}+\frac{u_{c \xi} \xi_{t}}{u_{c}} \hat{G}_{t}\right] \\
& -u_{c} \bar{Y} \frac{u_{c c} \bar{Y}}{u_{c}} \frac{\bar{G}_{\bar{Y}}}{\bar{Y}} \hat{Y}_{t}+\text { t.i.p. }+\mathcal{O}\left(\|\xi\|^{3}\right) \\
= & u_{c} \bar{Y}\left[\hat{Y}_{t}+\frac{1}{2}\left(1-\sigma^{-1}\right) \hat{Y}_{t}^{2}+\sigma^{-1} d_{t} \hat{Y}_{t}\right]- \\
& u_{c} \bar{G}\left[\hat{G}_{t}+\frac{1}{2}\left(1-\sigma^{-1} \gamma\right) \hat{G}_{t}^{2}+\sigma^{-1} d_{t} \hat{G}_{t}\right] \\
& +u_{c} \bar{Y} \sigma^{-1} \gamma \hat{Y}_{t} \hat{G}_{t}+t . i \cdot p .+\mathcal{O}\left(\|\xi\|^{3}\right)
\end{aligned}
$$

where $\sigma^{-1} \equiv-u_{c c} \bar{Y} / u_{c}, d_{t} \equiv \sigma u_{c \xi} \xi_{t} / u_{c}, \gamma \equiv \bar{G} / \bar{Y}$ and t.i.p. are the terms independent of policy.

The expansion of the second term gives: 


$$
\begin{aligned}
g\left(G_{t}, \xi_{t}\right) \approx \bar{g}+g_{G} \tilde{G}_{t}+g_{\xi} \xi_{t}+\frac{1}{2} g_{G G} \tilde{G}_{t}^{2}+g_{G \xi} \xi_{t} \tilde{G}_{t}+\frac{1}{2} \xi_{t}^{\prime} u_{\xi \xi} \xi_{t}+\mathcal{O}\left(\|\xi\|^{3}\right) \\
=\begin{array}{l}
\bar{g}+g_{G} \bar{G}\left(\hat{G}_{t}+\frac{1}{2} \hat{G}_{t}^{2}\right)+g_{\xi} \xi_{t}+\frac{1}{2} g_{G G} \bar{G}^{2}\left(\hat{G}_{t}+\frac{1}{2} \hat{G}_{t}^{2}\right)^{2}+ \\
g_{G \xi} \xi_{t} \bar{G}\left(\hat{G}_{t}+\frac{1}{2} \hat{G}_{t}^{2}\right)+\frac{1}{2} \xi_{t}^{\prime} u_{\xi \xi} \xi_{t}+\mathcal{O}\left(\|\xi\|^{3}\right)
\end{array} \\
=g_{G} \bar{G}\left(\hat{G}_{t}+\frac{1}{2} \hat{G}_{t}^{2}\right)+\frac{1}{2} g_{G G} \bar{G}^{2} \hat{G}_{t}^{2}+g_{G \xi} \bar{G} \xi_{t} \hat{G}_{t}+t . i . p .+\mathcal{O}\left(\|\xi\|^{3}\right) \\
=g_{G} \bar{G}\left[\hat{G}_{t}+\frac{1}{2} \hat{G}_{t}^{2}+\frac{1}{2} \frac{g_{G G} \bar{G}}{g_{G}} \hat{G}_{t}^{2}+\frac{g_{G \xi} \xi_{t}}{g_{G}} \hat{G}_{t}\right]+t . i . p .+\mathcal{O}\left(\|\xi\|^{3}\right) \\
=g_{G} \bar{G}\left[\hat{G}_{t}+\frac{1}{2}\left(1-\sigma_{G}^{-1}\right) \hat{G}_{t}^{2}+\sigma_{G}^{-1} d_{t}^{G} \hat{G}_{t}\right]+t . i . p .+\mathcal{O}\left(\|\xi\|^{3}\right)
\end{aligned}
$$

where $\sigma_{G}^{-1} \equiv-g_{G G} \bar{G} / g_{G}, d_{t}^{G} \equiv \sigma_{G} g_{G \xi} \xi_{t} / g_{G}$.

Finally, the expansion of the third term yields:

$$
\begin{gathered}
v\left(y_{t}(i), \xi_{t}\right) \approx \bar{v}+v_{y} \tilde{y}_{t}(i)+v_{\xi} \xi_{t}+\frac{1}{2} v_{y y} \tilde{y}_{t}(i)^{2}+v_{y \xi} \xi_{t} \tilde{y}_{t}(i)+\frac{1}{2} \xi_{t}^{\prime} v_{\xi \xi} \xi_{t}+\mathcal{O}\left(\|\xi\|^{3}\right) \\
=\begin{array}{l}
\bar{v}+v_{y} \bar{Y}\left(\hat{y}_{t}(i)+\frac{1}{2} \hat{y}_{t}(i)^{2}\right)+\frac{1}{2} v_{y y}\left[\bar{Y}\left(\hat{y}_{t}(i)+\frac{1}{2} \hat{y}_{t}(i)^{2}\right)\right]^{2}+ \\
v_{y \xi} \xi_{t} \bar{Y}\left(\hat{y}_{t}(i)+\frac{1}{2} \hat{y}_{t}(i)^{2}\right)+v_{\xi} \xi_{t}+\frac{1}{2} \xi_{t}^{\prime} v_{\xi \xi} \xi_{t}+\mathcal{O}\left(\|\xi\|^{3}\right)
\end{array} \\
=\bar{Y} v_{y} \hat{y}_{t}(i)+\frac{1}{2} \bar{Y}\left[v_{y}+v_{y y} \bar{Y}\right] \hat{y}_{t}(i)^{2}+v_{y \xi} \bar{Y} \xi_{t} \hat{y}_{t}(i)+t . i . p .+\mathcal{O}\left(\|\xi\|^{3}\right) \\
=\bar{Y} v_{y}\left\{\hat{y}_{t}(i)+\frac{1}{2}\left[1+\frac{v_{y y}}{v_{y}} \bar{Y}\right] \hat{y}_{t}(i)^{2}+\frac{v_{y \xi}}{v_{y}} \xi_{t} \hat{y}_{t}(i)\right\}+t . i . p .+\mathcal{O}\left(\|\xi\|^{3}\right)
\end{gathered}
$$

where $\hat{y}_{t}(i) \equiv \log \left(y_{t}(i) / \bar{Y}\right)$. We can use the first order conditions of the household's problem to get:

$$
\frac{v_{y}(\bar{Y}, \xi)}{u_{c}(\bar{Y}-\bar{G}, \xi)}=\frac{\theta-1}{\theta}\left(1-\bar{\tau}^{I}\right)=1-\Phi \Rightarrow v_{y}=(1-\Phi) u_{c}
$$

where $\frac{\theta-1}{\theta}$ is the desired mark up as a result of the suppliers market power. Woodford (2) explains that the parameter $\Phi>0$, which is assumed to be of order $\mathcal{O}(\|\xi\|)$, summarizes the overall distortion in the steady-state output level as a result of both taxes and market power. Substituting into 
the expansion above, we get:

$$
\begin{aligned}
& v\left(y_{t}(i), \xi_{t}\right)=\bar{Y}(1-\Phi) u_{c}\left\{\begin{array}{l}
\hat{y}_{t}(i)+ \\
\frac{1}{2}\left[1+\frac{\bar{Y} v_{y y}}{v_{y}}\right] \hat{y}_{t}(i)^{2}+ \\
\bar{Y} v_{y y} \\
\overline{\bar{Y} v_{y y}} \frac{v_{y \xi}}{v_{y}} \xi_{t} \hat{y}_{t}(i)
\end{array}\right\}+t . i . p .+\mathcal{O}\left(\|\xi\|^{3}\right) \\
& =\bar{Y} u_{c}\left\{\begin{array}{l}
(1-\Phi) \hat{y}_{t}(i)+\frac{1}{2}\left[1+\frac{\bar{Y} v_{y y}}{v_{y}}\right] \hat{y}_{t}(i)^{2}+ \\
\frac{\bar{Y} v_{y y}}{v_{y}} \frac{v_{y \xi} \xi_{t}}{\bar{Y} v_{y y}} \hat{y}_{t}(i)
\end{array}\right\}+t . i . p .+\mathcal{O}\left(\|\xi\|^{3}\right) \\
& =\bar{Y} u_{c}\left\{(1-\Phi) \hat{y}_{t}(i)+\frac{1}{2}[1+\omega] \hat{y}_{t}(i)^{2}-\omega q_{t} \hat{y}_{t}(i)\right\}+t . i . p .+\mathcal{O}\left(\|\xi\|^{3}\right)
\end{aligned}
$$

where $\omega=\bar{Y} v_{y y} / v_{y}$ and $q_{t} \equiv-v_{y \xi} \xi_{t} /\left(\bar{Y} v_{y y}\right)$. Integrating this expression over the differentiated good $i$ yields:

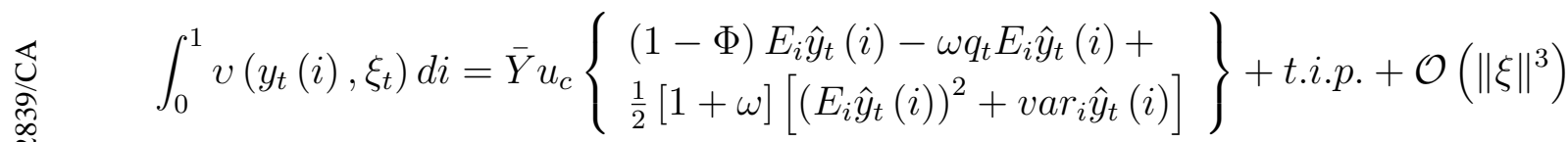

$$
\begin{aligned}
& =\bar{Y} u_{c}\left\{\begin{array}{l}
(1-\Phi)\left[\hat{Y}_{t}-\frac{1}{2}\left[1-\theta^{-1}\right] \operatorname{var}_{i} \hat{y}_{t}(i)\right]- \\
\omega q_{t}\left[\hat{Y}_{t}-\frac{1}{2}\left[1-\theta^{-1}\right] \operatorname{var}_{i} \hat{y}_{t}(i)\right]+ \\
\frac{1}{2}[1+\omega]\left[\left(\hat{Y}_{t}-\frac{1}{2}\left[1-\theta^{-1}\right] \operatorname{var}_{i} \hat{y}_{t}(i)\right)^{2}+\operatorname{var}_{i} \hat{y}_{t}(i)\right]
\end{array}\right\}+\text { t.i.p. }+\mathcal{O}\left(\|\xi\|^{3}\right) \\
& =\bar{Y} u_{c}\left\{\begin{array}{l}
(1-\Phi) \hat{Y}_{t}+\frac{1}{2}[1+\omega] \hat{Y}_{t}^{2}-\omega q_{t} \hat{Y}_{t} \\
-\frac{1}{2}\left[1-\theta^{-1}\right] \operatorname{var}_{i} \hat{y}_{t}(i)+\frac{1}{2}[1+\omega] \operatorname{var}_{i} \hat{y}_{t}(i)
\end{array}\right\}+\text { t.i.p. }+\mathcal{O}\left(\|\xi\|^{3}\right) \\
& =\bar{Y} u_{c}\left\{\begin{array}{l}
(1-\Phi) \hat{Y}_{t}+\frac{1}{2}[1+\omega] \hat{Y}_{t}^{2}-\omega q_{t} \hat{Y}_{t} \\
+\frac{1}{2}\left[\theta^{-1}+\omega\right] \operatorname{var}_{i} \hat{y}_{t}(i)
\end{array}\right\}+\text { t.i.p. }+\mathcal{O}\left(\|\xi\|^{3}\right)
\end{aligned}
$$

where $E_{i} \hat{y}_{t}(i)$ denotes the mean value of $\hat{y}_{t}(i)$ across all differentiated goods at date $t$ and $\operatorname{var}_{i} \hat{y}_{t}(i)$ is the corresponding variance. The last line makes use of the Taylor series approximation of the aggregate $Y_{t} \equiv\left[\int_{0}^{1} y_{t}(i)^{\frac{\theta-1}{\theta}} d i\right]^{\frac{\theta}{\theta-1}}$

$$
\hat{Y}_{t}=E_{i} \hat{y}_{t}(i)+\frac{1}{2}(1-\theta)^{-1} \operatorname{var}_{i} \hat{y}_{t}(i)+\mathcal{O}\left(\|\xi\|^{3}\right)
$$

to eliminate $E_{i} \hat{y}_{t}(i)$.

We can use that, in steady state, the marginal utility of consumption must be equal to the marginal utility of government spending $\left(u_{c}=g_{G}\right)$ and 
assume that the intertemporal elasticities of substitutions of public and private spending are equal $\left(\sigma=\sigma_{G}\right)$. Putting the first and second terms together:

$$
\begin{gathered}
u_{c} \bar{Y}\left[\hat{Y}_{t}+\frac{1}{2}\left(1-\sigma^{-1}\right) \hat{Y}_{t}^{2}+\sigma^{-1} d_{t} \hat{Y}_{t}\right]-u_{c} \bar{Y} \gamma\left[\hat{G}_{t}+\frac{1}{2}\left(1-\sigma^{-1} \gamma\right) \hat{G}_{t}^{2}+\sigma^{-1} d_{t} \hat{G}_{t}\right] \\
+u_{c} \bar{Y} \sigma^{-1} \gamma \hat{Y}_{t} \hat{G}_{t}+u_{c} \bar{Y} \gamma\left[\hat{G}_{t}+\frac{1}{2}\left(1-\sigma^{-1}\right) \hat{G}_{t}^{2}+\sigma^{-1} d_{t}^{G} \hat{G}_{t}\right]+t . i . p .+\mathcal{O}\left(\|\xi\|^{3}\right) \\
=\begin{array}{l}
u_{c} \bar{Y}\left[\hat{Y}_{t}+\frac{1}{2}\left(1-\sigma^{-1}\right) \hat{Y}_{t}^{2}+\sigma^{-1} d_{t} \hat{Y}_{t}\right]+u_{c} \bar{Y} \sigma^{-1} \gamma \hat{Y}_{t} \hat{G}_{t} \\
-u_{c} \bar{Y} \gamma\left[\frac{1}{2} \sigma^{-1}(1-\gamma) \hat{G}_{t}^{2}+\sigma^{-1}\left(d_{t}-d_{t}^{G}\right) \hat{G}_{t}\right]+t . i . p .+\mathcal{O}\left(\|\xi\|^{3}\right)
\end{array} \\
=u_{c} \bar{Y}\left\{\begin{array}{l}
\hat{Y}_{t}+\frac{1}{2}\left(1-\sigma^{-1}\right) \hat{Y}_{t}^{2}+\sigma^{-1} d_{t} \hat{Y}_{t} \\
+\sigma^{-1} \gamma \hat{Y}_{t} \hat{G}_{t}-\frac{\gamma \sigma^{-1}(1-\gamma)}{2} \hat{G}_{t}^{2}
\end{array}\right\}+t . i . p .+\mathcal{O}\left(\|\xi\|^{3}\right) \\
=u_{c} \bar{Y}\left\{\begin{array}{l}
\frac{1}{2}\left(1-\sigma^{-1}\right) \hat{Y}_{t}^{2}+\left(1+\sigma^{-1} d_{t}+\sigma^{-1} \gamma \hat{G}_{t}\right) \hat{Y}_{t} \\
-\frac{\gamma \sigma^{-1}(1-\gamma)}{2} \hat{G}_{t}^{2}
\end{array}\right\}+t . i . p .+\mathcal{O}\left(\|\xi\|^{3}\right)
\end{gathered}
$$

where I assume that the preference shock $\xi_{t}$ enters the utility function of private and public consumption in the same way, which implies $d_{t}=d_{t}^{G}$.

Combining this last expression with the third term we obtain:

$$
\begin{aligned}
& u_{c} \bar{Y}\left\{\frac{1}{2}\left(1-\sigma^{-1}\right) \hat{Y}_{t}^{2}+\left(1+\sigma^{-1} d_{t}+\sigma^{-1} \gamma \hat{G}_{t}\right) \hat{Y}_{t}-\frac{\gamma \sigma^{-1}(1-\gamma)}{2} \hat{G}_{t}^{2}\right\} \\
& U_{t}=-\bar{Y} u_{c}\left\{(1-\Phi) \hat{Y}_{t}+\frac{1}{2}[1+\omega] \hat{Y}_{t}^{2}-\omega q_{t} \hat{Y}_{t}+\frac{1}{2}\left[\theta^{-1}+\omega\right] \operatorname{var}_{i} \hat{y}_{t}(i)\right\} \\
& + \text { t.i.p. }+\mathcal{O}\left(\|\xi\|^{3}\right) \\
& =-\frac{u_{c} \bar{Y}}{2}\left\{\begin{array}{l}
\left(\sigma^{-1}+\omega\right) \hat{Y}_{t}^{2}-2\left[\sigma^{-1} d_{t}+\omega q_{t}+\sigma^{-1} \gamma \hat{G}_{t}+\Phi\right] \hat{Y}_{t} \\
+\gamma \sigma^{-1}(1-\gamma) \hat{G}_{t}^{2}+\left[\theta^{-1}+\omega\right] \operatorname{var}_{i} \hat{y}_{t}(i)
\end{array}\right\} \\
& + \text { t.i.p. }+\mathcal{O}\left(\|\xi\|^{3}\right)
\end{aligned}
$$

Assuming that the government removes monopolistic distortions and distortions from income taxation in the steady state through a subsidy $\mu^{w} \equiv$ $\frac{\theta-1}{\theta}\left(1-\bar{\tau}^{I}\right)$, then $\Phi=0$, which implies $v_{y}=u_{c}$. Consequently we have that $\sigma^{-1} d_{t}+\omega q_{t}=0$. This removes the linear terms in the expression above:

$$
\begin{aligned}
U_{t} & =-\frac{u_{c} \bar{Y}}{2}\left\{\begin{array}{l}
\left(\sigma^{-1}+\omega\right)\left[\hat{Y}_{t}^{2}-2 \frac{\sigma^{-1} \gamma}{\sigma^{-1}+\omega} \hat{G}_{t} \hat{Y}_{t}+\frac{\sigma^{-1} \gamma}{\sigma^{-1}+\omega}(1-\gamma) \hat{G}_{t}^{2}\right] \\
+\left[\theta^{-1}+\omega\right] \operatorname{var}_{i} \hat{y}_{t}(i)
\end{array}\right\} \\
& + \text { t.i.p. }+\mathcal{O}\left(\|\xi\|^{3}\right)
\end{aligned}
$$




$$
\begin{aligned}
U_{t} & =-\frac{u_{c} \bar{Y}}{2}\left\{\begin{array}{l}
\left(\sigma^{-1}+\omega\right)\left(\hat{Y}_{t}-\frac{\sigma^{-1} \gamma}{\sigma^{-1}+\omega} \hat{G}_{t}\right)^{2}+\left[(1-\gamma)-\frac{\sigma^{-1} \gamma}{\sigma^{-1}+\omega}\right] \sigma^{-1} \gamma \hat{G}_{t}^{2} \\
+\left[\theta^{-1}+\omega\right] \operatorname{var}_{i} \hat{y}_{t}(i)
\end{array}\right\} \\
& + \text { t.i.p. }+\mathcal{O}\left(\|\xi\|^{3}\right)
\end{aligned}
$$

The price dispersion term $\operatorname{var}_{i} \hat{y}_{t}(i)$ can be written as a function of inflation using the demand condition for good $i, y_{t}(i)=Y_{t}\left[p_{t}(i) / P_{t}\right]^{-\theta}$ which yields:

$$
\log y_{t}(i)=\log Y_{t}-\theta\left(\log p_{t}(i)-\log P_{t}\right)
$$

Thus,

$$
\operatorname{var}_{i} \log \hat{y}_{t}(i)=\theta^{2} \operatorname{var}_{i} \log p_{t}(i)
$$

Substituting into the expression for $U_{t}$ :

$$
U_{t}=-\frac{u_{c} \bar{Y}}{2}\left\{\begin{array}{c}
\left(\sigma^{-1}+\omega\right)\left(\hat{Y}_{t}-\frac{\sigma^{-1} \gamma}{\sigma^{-1}+\omega} \hat{G}_{t}\right)^{2} \\
+\left[(1-\gamma)-\frac{\sigma^{-1} \gamma}{\sigma^{-1}+\omega}\right] \sigma^{-1} \gamma \hat{G}_{t}^{2} \\
+\theta[1+\omega \theta] \operatorname{var}_{i} \log p_{t}(i)
\end{array}\right\}+t . i . p .+\mathcal{O}\left(\|\xi\|^{3}\right)
$$

Define $\Delta_{t} \equiv v a r_{i} \log p_{t}(i)$, which can be written in recursive form as

$$
\Delta_{t}=\alpha \Delta_{t-1}+\frac{\alpha}{1-\alpha} \pi_{t}^{2}+\mathcal{O}\left(\|\xi\|^{3}\right)
$$

Iterating backwards to time $t=0$ :

$$
\Delta_{t}=\alpha^{t+1} \Delta_{-1}+\frac{\alpha}{1-\alpha} \sum_{s=0}^{t} \alpha^{t-s} \pi_{s}^{2}+\mathcal{O}\left(\|\xi\|^{3}\right)
$$

Taking the discounted value of these terms $\forall t \geq 0$ we obtain:

$$
\sum_{t=0}^{\infty} \beta^{t} \Delta_{t}=\frac{\alpha}{(1-\alpha)(1-\alpha \beta)} \sum_{t=0}^{\infty} \beta^{t} \pi_{t}^{2}+\text { t.i.p. }+\mathcal{O}\left(\|\xi\|^{3}\right)
$$

Substitute it back in the loss function and rearranging:

$$
\sum_{t=0}^{\infty} \beta^{t} U_{t}=-\frac{\bar{Y} u_{c}}{2} \sum_{t=0}^{\infty} \beta^{t}\left\{\begin{array}{l}
\frac{\alpha \theta(1+\omega \theta)}{(1-\alpha)(1-\alpha \beta)} \pi_{t}^{2} \\
+\left(\sigma^{-1}+\omega\right)\left(\hat{Y}_{t}-\frac{\sigma^{-1} \gamma}{\sigma^{-1}+\omega} \hat{G}_{t}\right)^{2} \\
+\left[(1-\gamma)-\frac{\sigma^{-1} \gamma}{\sigma^{-1}+\omega}\right] \sigma^{-1} \gamma \hat{G}_{t}^{2}
\end{array}\right\}+\text { t.i.p. }+\mathcal{O}\left(\|\xi\|^{3}\right)
$$




$$
\begin{aligned}
& \sum_{t=0}^{\infty} \beta^{t} U_{t}=-\frac{\bar{Y} u_{c}}{2} \frac{\alpha \theta(1+\omega \theta)}{(1-\alpha)(1-\alpha \beta)} \times
\end{aligned}
$$

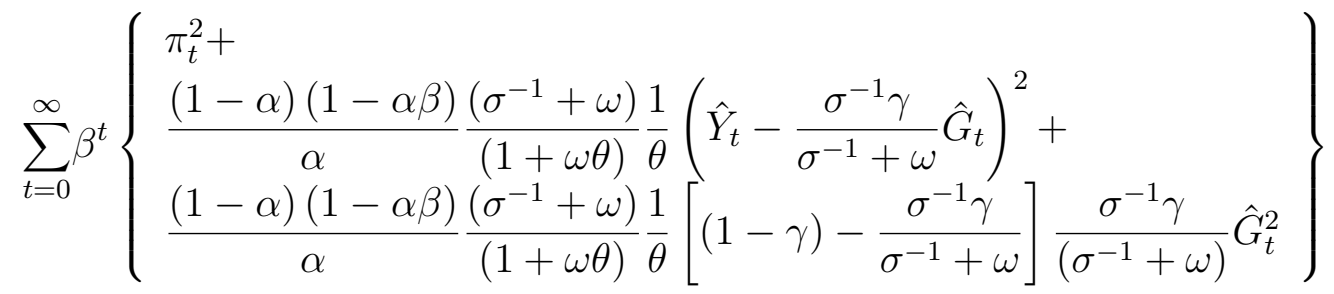

$$
\begin{aligned}
& + \text { t.i.p. }+\mathcal{O}\left(\|\xi\|^{3}\right) \\
& \text { Define } \Omega \equiv \frac{\bar{Y} u_{c}}{2} \frac{\alpha \theta(1+\omega \theta)}{(1-\alpha)(1-\alpha \beta)}, \kappa \equiv \frac{(1-\alpha)(1-\alpha \beta)}{\alpha} \frac{\left(\sigma^{-1}+\omega\right)}{(1+\omega \theta)} \text { and } \\
& \Gamma \equiv \frac{\sigma^{-1} \gamma}{\sigma^{-1}+\omega} \\
& \sum_{t=0}^{\infty} \beta^{t} U_{t}=-\Omega \sum_{t=0}^{\infty} \beta^{t}\left\{\pi_{t}^{2}+\lambda_{y}\left(\hat{Y}_{t}-\Gamma \hat{G}_{t}\right)^{2}+\lambda_{G} \hat{G}_{t}^{2}\right\}+\text { t.i.p. }+\mathcal{O}\left(\|\xi\|^{3}\right)
\end{aligned}
$$

where $\lambda_{y} \equiv \frac{\kappa}{\theta}$ and $\lambda_{g} \equiv \lambda_{y} \Gamma[1-\gamma-\Gamma]$. This loss function is similar to that presented by Woodford (33).

Proof. (Proposition 3.1 - Part II - PDV Welfare Loss Function) The probability structure of the model can be used to write the infinite summation in the loss function in terms of present discounted values. Before the crisis, the economy is at the steady state, where $\left(\pi_{t}=\hat{Y}_{t}=\hat{G}_{t}=0\right)$, so welfare losses will be zero until the crisis hits. At the initial crisis state $\left(t=T_{0}\right)$, this function assumes the value $S=\left[\pi_{S}^{2}+\lambda_{y}\left(Y_{S}-\Gamma G_{S}\right)^{2}+\lambda_{g} G_{S}^{2}\right]$. In the following period, with probability $\mu$, the shock will still be effective, and the economy will have welfare $S$ again. With probability $(1-\mu)(1-b)$ it will go to the long run ${ }^{1}$ and welfare will be $L=\left[\pi_{L}^{2}+\lambda_{y}\left(Y_{L}-\Gamma G_{L}\right)^{2}+\lambda_{g} G_{L}^{2}\right]$. With probability $(1-\mu) b$ there will be a transitional state due to a combination of monetary and fiscal policies, even when the zero lower bound is no longer binding. In this state, welfare will assume the value ${ }^{2} M=\left[\pi_{M}^{2}+\lambda_{y}\left(Y_{M}-\Gamma G_{M}\right)^{2}+\lambda_{g} G_{M}^{2}\right]$. After the economy enters the transitional state, it is expected to stay there in the

${ }^{1}$ In order to obtain a general expression for the function, it is assumed that the economy does not necessarily go back to the steady state in the long run. This happens when policies implemented during the crisis are expected to be permanent. If they are temporary, the longrun allocations go back to $\left(\pi_{L}=Y_{L}=G_{L}=0\right)$, and it is possible to simplify the expression even more.

${ }^{2}$ Note that the medium-run values for output, inflation and government spending here will be different depending on the combination of monetary and fiscal policies chosen. But to derive the present value of welfare losses their actual values do not matter at this point. 
following period with probability $b$, with welfare losses $M$. With probability $(1-b)$ the economy goes to the long run and welfare will be $L$.

Expanding the summation of the loss function for a few periods using the definitions for $S, M$ and $L$, we can analyze the pattern:

$$
\begin{aligned}
& L^{P D V}=\underbrace{S}_{t=0}+\underbrace{\beta\left[\begin{array}{c}
\mu S+(1-\mu) b M+ \\
(1-\mu)(1-b) L
\end{array}\right]}_{t=1}+\underbrace{\beta^{2}\left[\begin{array}{c}
\mu^{2} S+(1-\mu) b[\mu+b] M+ \\
(1-\mu)(1-b)[1+\mu+b] L
\end{array}\right]}_{t=2}+ \\
& \underbrace{\beta^{3}\left[\begin{array}{l}
\mu^{3} S+(1-\mu) b\left[\mu^{2}+b^{2}+\mu b\right] M+ \\
(1-\mu)(1-b)\left[1+\mu+\mu^{2}+b+b^{2}+\mu b\right] L
\end{array}\right]}_{t=3}+ \\
& \underbrace{\beta^{4}\left[\begin{array}{l}
\mu^{4} S+(1-\mu) b\left[\mu^{3}+b^{3}+\mu b(\mu+b)\right] M+ \\
(1-\mu)(1-b)\left[1+\mu+\mu^{2}+\mu^{3}+b+b^{2}+b^{3}+\mu b(1+\mu+b)\right]
\end{array}\right]}_{t=4}+ \\
& \underbrace{\beta^{5}\left[\begin{array}{l}
\mu^{5} S+(1-\mu) b\left[\mu^{4}+b^{4}+\mu b\left(\mu b+\mu^{2}+b^{2}\right)\right] M+ \\
(1-\mu)(1-b)\left[\begin{array}{l}
1+\mu+\mu^{2}+\mu^{3}+\mu^{4}+b+b^{2}+b^{3}+b^{4}+ \\
\mu b\left(1+\mu+\mu^{2}+b+b^{2}+\mu b\right)
\end{array}\right]
\end{array}\right]}_{t=5}+ \\
& \underbrace{\beta^{6}\left[\begin{array}{c}
\mu^{6} S+(1-\mu) b\left[\mu^{5}+b^{5}+\mu b\left(\mu^{3}+b^{3}+\mu b(\mu+b)\right)\right] M \\
(1-\mu)(1-b)\left[\begin{array}{c}
1+\mu+\mu^{2}+\mu^{3}+\mu^{4}+\mu^{5}+b+b^{2}+b^{3}+b^{4}+b^{5}+ \\
\mu b\left(1+\mu+\mu^{2}+\mu^{3}+b+b^{2}+b^{3}+\mu b(1+\mu+b)\right)
\end{array}\right]
\end{array}\right]}_{t=6}+\cdots
\end{aligned}
$$

Distributing the $\beta^{\prime} s$ and grouping similar terms:

$$
\begin{aligned}
& L^{P D V}=\left(1+\beta \mu+\beta^{2} \mu^{2}+\beta^{3} \mu^{3}+\beta^{4} \mu^{4}+\beta^{5} \mu^{5}+\beta^{6} \mu^{6}+\beta^{7} \mu^{7}+\cdots\right) S+ \\
& \left\{\beta(1-\mu) b\left[\begin{array}{l}
1+\beta[\mu+b]+\beta^{2}\left[\mu^{2}+b^{2}+\mu b\right]+ \\
\beta^{3}\left[\mu^{3}+b^{3}+\mu b(\mu+b)\right]+ \\
\beta^{4}\left[\mu^{4}+b^{4}+\mu b\left(\mu b+\mu^{2}+b^{2}\right)\right]+ \\
\beta^{5}\left[\mu^{5}+b^{5}+\mu b\left(\mu^{3}+b^{3}+\mu b(\mu+b)\right)\right]+ \\
\beta^{6}\left[\mu^{6}+b^{6}+\mu b\left(\mu^{4}+b^{4}+\mu b\left(\mu^{2}+b^{2}+\mu b\right)\right)\right]+\ldots
\end{array}\right]\right\} M+ \\
& \left\{(1-\mu)(1-b)\left[\begin{array}{l}
1+\beta[1+\mu+b]+\beta^{2}\left[1+\mu+\mu^{2}+b+b^{2}+\mu b\right]+ \\
\left.\beta^{3}\left[\begin{array}{l}
\left.1+\mu+\mu^{2}+\mu^{3}+b+b^{2}+b^{3}+\mu b(1+\mu+b)\right]+ \\
\beta^{4}\left[\begin{array}{l}
1+\mu+\mu^{2}+\mu^{3}+\mu^{4}+b+b^{2}+b^{3}+b^{4}+ \\
\mu b\left(1+\mu+\mu^{2}+b+b^{2}+\mu b\right)
\end{array}\right]+ \\
\beta^{5}\left[\begin{array}{l}
1+\mu+\mu^{2}+\mu^{3}+\mu^{4}+\mu^{5}+ \\
b+b^{2}+b^{3}+b^{4}+b^{5}+ \\
\mu b\left(\begin{array}{l}
1+\mu+\mu^{2}+\mu^{3}+ \\
b+b^{2}+b^{3}+\mu b(1+\mu+b)
\end{array}\right)
\end{array}\right]+ \\
\beta^{6}\left[\begin{array}{l}
1+\mu+\mu^{2}+\mu^{3}+\mu^{4}+\mu^{5}+\mu^{6}+ \\
b+b^{2}+b^{3}+b^{4}+b^{5}+b^{6} \\
\mu\left(\begin{array}{l}
1+\mu+\mu^{2}+\mu^{3}+\mu^{4}+ \\
b+b^{2}+b^{3}+b^{4}+ \\
\mu b\left(1+\mu+\mu^{2}+b+b^{2}+\mu b\right)
\end{array}\right)+\cdots
\end{array}\right]+\cdots
\end{array}\right]\right)
\end{array}\right]\right. \text { L }
\end{aligned}
$$




\section{Rearranging:}

$$
L^{P D V}=\left(\begin{array}{l}
1+\beta \mu+\beta^{2} \mu^{2}+ \\
\beta^{3} \mu^{3}+\beta^{4} \mu^{4}+\beta^{5} \mu^{5}+ \\
\beta^{6} \mu^{6}+\beta^{7} \mu^{7}+\cdots
\end{array}\right) S+
$$

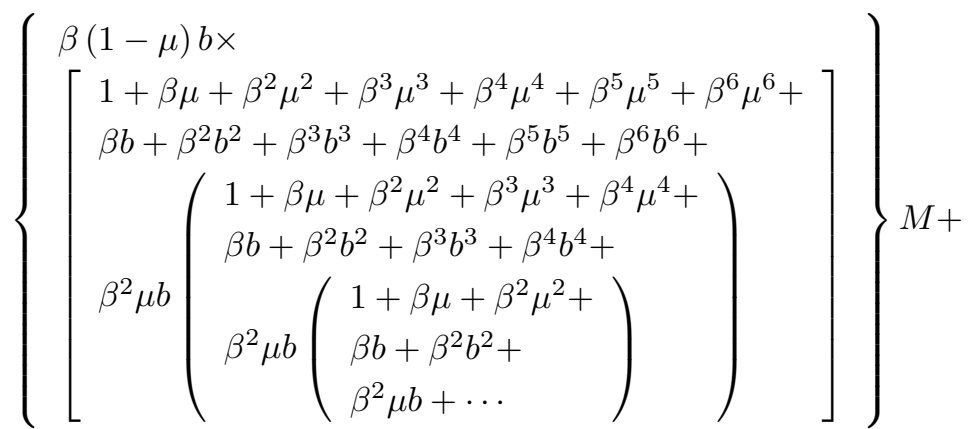


Grouping similar terms one more time:

$$
\begin{aligned}
L^{P D V}= & \left(1+\beta \mu+\beta^{2} \mu^{2}+\beta^{3} \mu^{3}+\beta^{4} \mu^{4}+\beta^{5} \mu^{5}+\beta^{6} \mu^{6}+\beta^{7} \mu^{7}+\cdots\right) S+ \\
& \left\{\begin{array}{l}
\beta(1-\mu) b\left(1+\beta^{2} \mu b+\left(\beta^{2} \mu b\right)^{2}+\cdots\right) \times \\
\\
\left(1+\beta \mu+\beta^{2} \mu^{2}+\beta^{3} \mu^{3}+\beta^{4} \mu^{4}+\beta^{5} \mu^{5}+\beta^{6} \mu^{6}+\cdots\right)+ \\
\beta b\left(1+\beta b+\beta^{2} b^{2}+\beta^{3} b^{3}+\beta^{4} b^{4}+\beta^{5} b^{5}+\cdots\right)
\end{array}\right] M+ \\
& \left\{\begin{array}{l}
\beta(1-\mu)(1-b)\left(1+\beta+\beta^{2}+\beta^{3}+\beta^{4}+\beta^{5}+\beta^{6}+\cdots\right) \times \\
\left(1+\beta^{2} \mu b+\left(\beta^{2} \mu b\right)^{2}+\cdots\right) \times \\
{\left[\begin{array}{l}
1+\beta \mu\left(1+\beta \mu+(\beta \mu)^{2}+(\beta \mu)^{3}+(\beta \mu)^{4}+\cdots\right)+ \\
\beta b\left(1+\beta b+(\beta b)^{2}+(\beta b)^{3}+(\beta b)^{4}+\cdots\right)+
\end{array}\right.}
\end{array}\right\} L
\end{aligned}
$$

We can write the summations as:

$$
\begin{aligned}
L^{P D V} & =\left[\sum_{t=0}^{\infty}(\beta \mu)^{t}\right] S+ \\
& \left\{\beta(1-\mu) b\left(\sum_{t=0}^{\infty}\left(\beta^{2} \mu b\right)^{t}\right)\left[\left(\sum_{t=0}^{\infty}(\beta \mu)^{t}\right)+\left(\beta b \sum_{t=0}^{\infty}(\beta b)^{t}\right)\right]\right\} M \\
& \left\{\begin{array}{l}
\beta(1-\mu)(1-b)\left(\sum_{t=0}^{\infty} \beta^{t}\right)\left(\sum_{t=0}^{\infty}\left(\beta^{2} \mu b\right)^{t}\right) \times \\
{\left[1+\beta \mu\left(\sum_{t=0}^{\infty}(\beta \mu)^{t}\right)+\beta b\left(\sum_{t=0}^{\infty}(\beta b)^{t}\right)\right]}
\end{array}\right\} L
\end{aligned}
$$

Using the properties of infinite summations to get a more straightforward expression:

$$
\begin{gathered}
L^{P D V}=\frac{1}{1-\beta \mu} S+\left\{\frac{\beta(1-\mu) b}{1-\beta^{2} \mu b}\left[\frac{1}{1-\beta \mu}+\frac{\beta b}{1-\beta b}\right]\right\} M+ \\
\left\{\frac{\beta}{1-\beta} \frac{(1-\mu)(1-b)}{1-\beta^{2} \mu b}\left[1+\frac{\beta \mu}{1-\beta \mu}+\frac{\beta b}{1-\beta b}\right]\right\} L
\end{gathered}
$$

Which gives the final expression for the welfare loss function in present discounted value terms:

$$
\begin{aligned}
L^{P D V}= & \frac{1}{1-\beta \mu}\left(\pi_{S}^{2}+\lambda_{y}\left(Y_{S}-\Gamma G_{S}\right)^{2}+\lambda_{g} G_{S}^{2}\right)+ \\
& \frac{\beta(1-\mu) b}{(1-\beta \mu)(1-\beta b)}\left(\pi_{M}^{2}+\lambda_{y}\left(Y_{M}-\Gamma G_{M}\right)^{2}+\lambda_{g} G_{M}^{2}\right)+ \\
& \frac{\beta(1-\mu)(1-b)}{(1-\beta)(1-\beta \mu)(1-\beta b)}\left(\pi_{L}^{2}+\lambda_{y}\left(Y_{L}-\Gamma G_{L}\right)^{2}+\lambda_{g} G_{L}^{2}\right)
\end{aligned}
$$




\section{C.2}

\section{Proof of Proposition 3.2}

Proposition C.2 (Proposition 3.2) Assuming Conditions $(C 1)-(C 4)$ hold, if the monetary authority keeps the nominal interest rate fixed at an optimal level $i_{M}^{*}$ in the transitional state, it picks this level by solving the following minimization problem

$$
\begin{aligned}
\min _{\left\{i_{M}\right\}} & L^{P D V} \\
\text { s.t. } & Y_{S}, \pi_{S}, Y_{M}, \pi_{M}, Y_{L}, \pi_{L} \\
& i_{M} \geq 0
\end{aligned}
$$

where $L^{P D V}$ is defined in Proposition 3.1 and the levels of output and inflation in each state are given by Proposition 2.1. The solution to this problem yields an optimal nominal interest rate given by

$$
\begin{gathered}
i_{M}^{*}=\left\{\begin{aligned}
i_{M}^{o p t}, & \text { if } i_{M}^{o p t}>0 \\
0, & \text { otherwise }
\end{aligned}\right. \\
i_{M}^{\text {opt }}=\bar{r}+\frac{1}{\Omega_{i_{M}, \bar{r}}^{*}}\left\{\begin{array}{l}
\Omega_{i_{M}, r_{S}^{e}}^{*} r_{S}^{e}+\Omega_{i_{M}, G_{S}}^{*} G_{S}+\Omega_{i_{M}, \tau_{S}^{I}}^{*} \tau_{S}^{I}+ \\
\Omega_{i_{M}, G_{M}}^{*} G_{M}+\Omega_{i_{M}, \tau_{M}^{I}}^{*} \tau_{M}^{I}
\end{array}\right\}
\end{gathered}
$$

where the analytical expressions for the coefficients $\Omega_{i_{M}, j}^{*}, j \in$ $\left\{\bar{r}, r_{S}^{e}, G_{S}, \tau_{S}^{I}, G_{M}, \tau_{M}^{I}\right\}$, are defined in the appendix and depend on the structural parameters and the coefficients $\left(\Omega^{\prime} s\right)$ from Proposition 2.1.

Proof. Since the long-run allocations do not depend on the medium-run nominal interest rate, the problem that the monetary authority needs to solve can be expressed as:

$$
\begin{aligned}
\min _{\left\{i_{M}\right\}} & 1-\beta \mu
\end{aligned}\left\{\begin{array}{l}
\left(\pi_{S}^{2}+\lambda_{y}\left(Y_{S}-\Gamma G_{S}\right)^{2}+\lambda_{g} G_{S}^{2}\right)+ \\
\frac{\beta(1-\mu) b}{(1-\beta b)}\left(\pi_{M}^{2}+\lambda_{y}\left(Y_{M}-\Gamma G_{M}\right)^{2}+\lambda_{g} G_{M}^{2}\right)
\end{array}\right\}
$$


Substituting the restrictions into the objective function:

$$
\begin{aligned}
& \min _{\left\{i_{M}\right\}} \frac{1}{1-\beta \mu}\left[\begin{array}{l}
\left(\begin{array}{l}
\Omega_{\pi_{S}, r_{S}^{e}} r_{S}^{e}+\Omega_{\pi_{S}, G_{S}} G_{S}+\Omega_{\pi_{S}, \tau_{S}^{I}} \tau_{S}^{I}+ \\
\Omega_{\pi_{S}, i_{M}}\left(\bar{r}-i_{M}\right)+\Omega_{\pi_{S}, G_{M}} G_{M}+\Omega_{\pi_{S}, \tau_{M}^{I}} \tau_{M}^{I}
\end{array}\right)^{2}+ \\
\lambda_{y}\left(\begin{array}{l}
\Omega_{Y_{S}, r_{S}^{e}} r_{S}^{e}+\left(\Omega_{Y_{S}, G_{S}}-\Gamma\right) G_{S}+\Omega_{Y_{S}, \tau_{S}^{I}} \tau_{S}^{I}+ \\
\Omega_{Y_{S}, i_{M}}\left(\bar{r}-i_{M}\right)+\Omega_{Y_{S}, G_{M}} G_{M}+\Omega_{Y_{S}, \tau_{M}^{I}} \tau_{M}^{I}
\end{array}\right)^{2}+ \\
\lambda_{g} G_{S}^{2}
\end{array}\right] \\
& +\frac{\beta(1-\mu) b}{(1-\beta \mu)(1-\beta b)} \times \\
& {\left[\begin{array}{l}
\left(\Omega_{\pi_{M}, i_{M}}\left(\bar{r}-i_{M}\right)+\Omega_{\pi_{M}, G_{M}} G_{M}+\Omega_{\pi_{M}, \tau_{M}^{I}} \tau_{M}^{I}\right)^{2}+ \\
\lambda_{y}\left(\Omega_{Y_{M}, i_{M}}\left(\bar{r}-i_{M}\right)+\left(\Omega_{Y_{M}, G_{M}}-\Gamma\right) G_{M}+\Omega_{Y_{M}, \tau_{M}^{I}} \tau_{M}^{I}\right)^{2}+ \\
\lambda_{g} G_{M}^{2}
\end{array}\right]}
\end{aligned}
$$

The first order condition with respect to $i_{M}$ is given by:

$$
\begin{aligned}
& -\frac{1}{1-\beta \mu}\left(\begin{array}{l}
\Omega_{\pi_{S}, r_{S}^{e}} r_{S}^{e}+\Omega_{\pi_{S}, G_{S}} G_{S}+\Omega_{\pi_{S}, \tau_{S}^{I}} \tau_{S}^{I}+ \\
\Omega_{\pi_{S}, i_{M}}\left(\bar{r}-i_{M}\right)+\Omega_{\pi_{S}, G_{M}} G_{M}+\Omega_{\pi_{S}, \tau_{M}^{I}} \tau_{M}^{I}
\end{array}\right) \Omega_{\pi_{S}, i_{M}} \\
& -\frac{1}{1-\beta \mu} \lambda_{y}\left(\begin{array}{c}
\Omega_{Y_{S}, r_{S}^{e}} r_{S}^{e}+\left(\Omega_{Y_{S}, G_{S}}-\Gamma\right) G_{S}+\Omega_{Y_{S}, \tau_{S}^{I}} \tau_{S}^{I}+ \\
\Omega_{Y_{S}, i_{M}}\left(\bar{r}-i_{M}\right)+\Omega_{Y_{S}, G_{M}} G_{M}+\Omega_{Y_{S}, \tau_{M}^{I}} \tau_{M}^{I}
\end{array}\right) \Omega_{Y_{S}, i_{M}} \\
& -\frac{\beta(1-\mu) b}{(1-\beta \mu)(1-\beta b)}\left(\Omega_{\pi_{M}, i_{M}}\left(\bar{r}-i_{M}\right)+\Omega_{\pi_{M}, G_{M}} G_{M}+\Omega_{\pi_{M}, \tau_{M}^{I}} \tau_{M}^{I}\right) \Omega_{\pi_{M}, i_{M}} \\
& -\frac{\beta(1-\mu) b}{(1-\beta \mu)(1-\beta b)} \lambda_{y} \times \\
& \left(\Omega_{Y_{M}, i_{M}}\left(\bar{r}-i_{M}\right)+\left(\Omega_{Y_{M}, G_{M}}-\Gamma\right) G_{M}+\Omega_{Y_{M}, \tau_{M}^{I} \tau_{M}^{I}}^{I}\right) \Omega_{Y_{M}, i_{M}}=0
\end{aligned}
$$

which can be written as:

$$
\begin{aligned}
& i_{M}^{o p t}=\bar{r}+\frac{1}{\Omega_{i_{M}, \bar{r}}^{*}}\left\{\Omega_{i_{M}, r_{S}^{e}}^{*} r_{S}^{e}+\Omega_{i_{M}, G_{S}}^{*} G_{S}+\Omega_{i_{M}, \tau_{S}^{I}}^{*} \tau_{S}^{I}+\Omega_{i_{M}, G_{M}}^{*} G_{M}+\Omega_{i_{M}, \tau_{M}^{I}}^{*} \tau_{M}^{I}\right\} \\
& \text { where } \Omega_{i_{M}, \bar{r}}^{*} \equiv\left[\begin{array}{l}
\left(\Omega_{\pi_{S}, i_{M}}\right)^{2}+\lambda_{y}\left(\Omega_{Y_{S}, i_{M}}\right)^{2}+ \\
\frac{\beta(1-\mu) b}{(1-\beta b)}\left(\left(\Omega_{\pi_{M}, i_{M}}\right)^{2}+\lambda_{y}\left(\Omega_{Y_{M}, i_{M}}\right)^{2}\right)
\end{array}\right] \text {, } \\
& \Omega_{i_{M}, r_{S}^{e}}^{*} \equiv\left[\Omega_{\pi_{S}, i_{M}} \Omega_{\pi_{S}, r_{S}^{e}}+\lambda_{y} \Omega_{Y_{S}, i_{M}} \Omega_{Y_{S}, r_{S}^{e}}\right] \text {, } \\
& \Omega_{i_{M}, G_{S}}^{*} \equiv\left[\Omega_{\pi_{S}, i_{M}} \Omega_{\pi_{S}, G_{S}}+\lambda_{y} \Omega_{Y_{S}, i_{M}}\left(\Omega_{Y_{S}, G_{S}}-\Gamma\right)\right], \\
& \Omega_{i_{M}, \tau_{S}^{I}}^{*} \equiv\left[\Omega_{\pi_{S}, i_{M}} \Omega_{\pi_{S}, \tau_{S}^{I}}+\lambda_{y} \Omega_{Y_{S}, i_{M}} \Omega_{Y_{S}, \tau_{S}^{I}}\right] \text {, } \\
& \Omega_{i_{M}, G_{M}}^{*} \equiv\left[\begin{array}{l}
\Omega_{\pi_{S}, i_{M}} \Omega_{\pi_{S}, G_{M}}+\lambda_{y} \Omega_{Y_{S}, i_{M}} \Omega_{Y_{S}, G_{M}}+ \\
\frac{\beta(1-\mu) b}{(1-\beta b)}\left(\Omega_{\pi_{M}, i_{M}} \Omega_{\pi_{M}, G_{M}}+\lambda_{y} \Omega_{Y_{M}, i_{M}}\left(\Omega_{Y_{M}, G_{M}}-\Gamma\right)\right)
\end{array}\right] \text { and } \\
& \Omega_{i_{M}, \tau_{M}^{I}}^{*} \equiv\left[\begin{array}{l}
\Omega_{\pi_{S}, i_{M}} \Omega_{\pi_{S}, \tau_{M}^{I}}+\lambda_{y} \Omega_{Y_{S}, i_{M}} \Omega_{Y_{S}, \tau_{M}^{I}}+ \\
\frac{\beta(1-\mu) b}{(1-\beta b)}\left(\Omega_{\pi_{M}, i_{M}} \Omega_{\pi_{M}, \tau_{M}^{I}}+\lambda_{y} \Omega_{Y_{M}, i_{M}} \Omega_{Y_{M}, \tau_{M}^{I}}\right)
\end{array}\right] .
\end{aligned}
$$


Thus, to also satisfy the non-negativity condition $\left(i_{M} \geq 0\right)$, we have that the optimal nominal interest rate is given by

$$
i_{M}^{*}=\left\{\begin{array}{rr}
i_{M}^{o p t} & , \text { if } i_{M}^{o p t}>0 \\
0 & , \text { otherwise }
\end{array}\right.
$$

